\title{
Balancing animal-source food intake between nutritional requirements and sustainability impacts
}

\section{Anita Frehner}

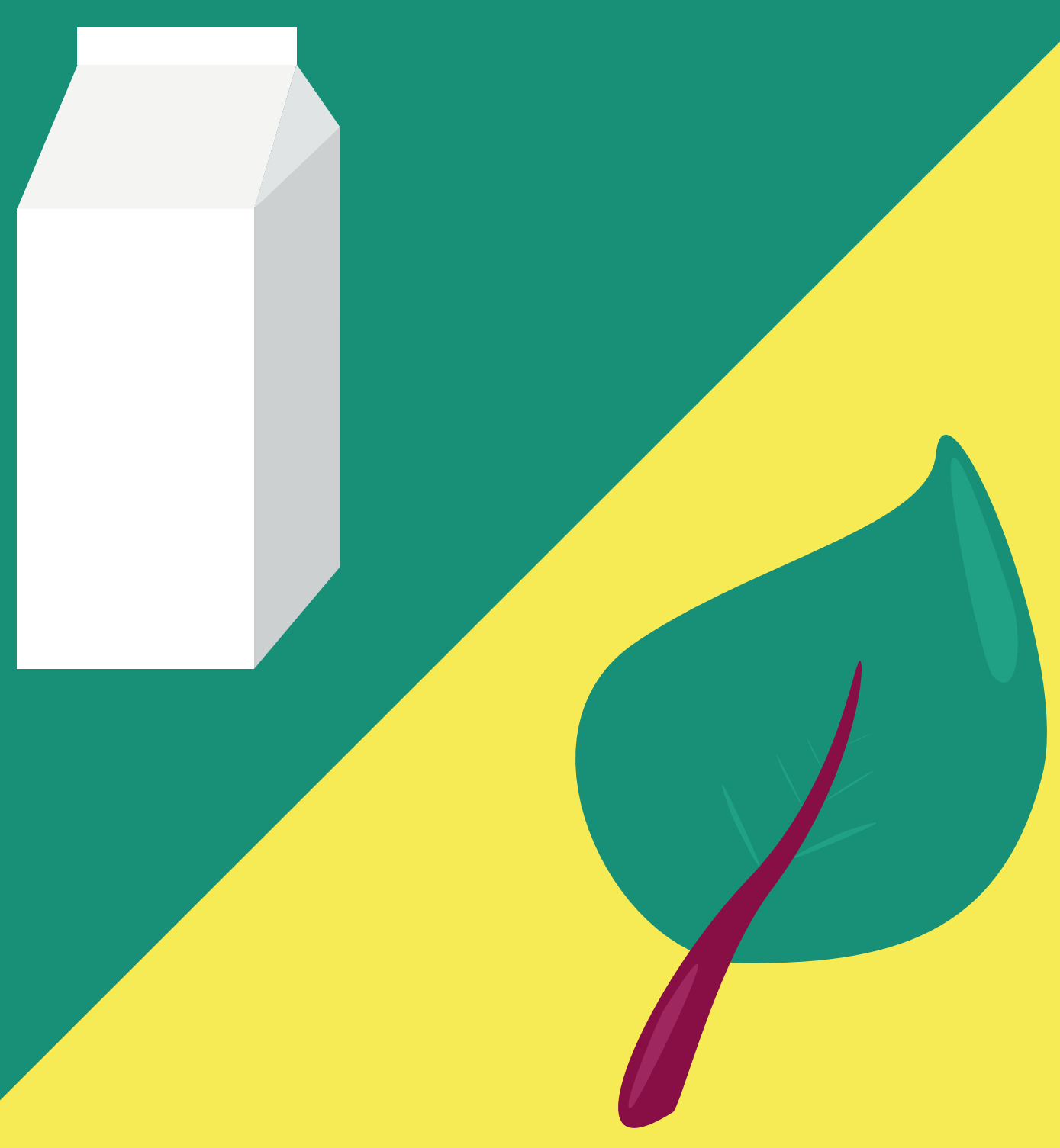





\section{Propositions}

1. Natural resources should first be allocated to provide nutritious food for humans rather than to feed farm animals.

(this thesis)

2. Current environmental footprints cannot capture the environmental consequences of large-scale food system changes.

(this thesis)

3. Fermentation has the potential to re-revolutionise plant-based food processing.

4. The request for more precision hampers scientists to formulate clear messages based on already existing evidence.

5. Consumerism counteracts sustainable development.

6. Excursions to utopias, such as Moominvalley, cultivate people's kindness.

Propositions belonging to the thesis, entitled

‘Balancing animal-source food intake between nutritional requirements and sustainability impacts'

Anita Frehner

Wageningen, 30 June 2021 



\title{
Balancing animal-source food intake between nutritional requirements and sustainability impacts
}

\author{
Anita Frehner
}




\section{Thesis committee}

\section{Promoter}

Prof. Dr I.J.M. de Boer

Professor of Animal Production Systems

Wageningen University \& Research

\section{Co-promoters}

Dr H.H.E. van Zanten

Associate professor, Farming Systems Ecology Group

Wageningen University \& Research

Dr A. Muller

Senior scientist

Research Institute of Organic Agriculture FiBL, Frick, Switzerland

Federal Institute of Technology Zurich ETHZ, Zurich, Switzerland

Dr C. Schader

Senior scientist

Research Institute of Organic Agriculture FiBL, Frick, Switzerland

\section{Other members}

Prof. Dr P.W.G. Groot Koerkamp, Wageningen University \& Research

Dr I.D. Brouwer, Wageningen University \& Research

Dr A. Kanellopoulos, Wageningen University \& Research

Dr A. Mayer, University of Natural Resources and Life Sciences, Vienna, Austria

This research was conducted under the auspices of the Graduate School of Wageningen Institute of Animal Science (WIAS) 


\title{
Balancing animal-source food intake between nutritional requirements and sustainability impacts
}

\author{
Anita Frehner
}

\section{Thesis}

submitted in fulfilment of the requirement for the degree of doctor

$$
\text { at Wageningen University }
$$

by the authority of the Rector Magnificus,

$$
\text { Prof. Dr A.J.P. Mol, }
$$

in the presence of the

Thesis Committee appointed by the Academic Board

to be defended in public

on Wednesday 30 June 2021

at 11 a.m. in the Aula. 
Frehner, Anita

Balancing animal-source food intake between nutritional requirements and sustainability impacts, 174 pages.

PhD thesis, Wageningen University, Wageningen, the Netherlands (2021)

With references, with summaries in English and German

DOI: https://doi.org/10.18174/543918

ISBN: 978-94-6395-755-7 


\section{Abstract}

The current environmental, social, and economic challenges of food systems necessitate actions towards more sustainable consumption patterns and production approaches. Reducing animalsource food (ASF) intake is a promising strategy to solve multiple sustainability issues. However, how many and which animals could be part of a sustainable food system remains up for discussion, as well as how the remaining ASF should best be produced. This thesis aimed to investigate the role of ASF in current and potential future food systems, with special emphasis on integrating different sustainability impacts. We conducted a literature review to understand why certain ASF are proposed as solutions for more sustainable diets. There, we found that studies considering statusquo impact assessments and specifying consumption-oriented scenarios recommend to reduce beef and pork rather than chicken. In these studies, the role of ASF is mostly limited to current production practices and feed-food competition is not addressed. Studies considering systemic consequences and specifying resource-oriented scenarios propose larger reductions of chicken and pork, because these are produced with more food-competing feed. In these latter studies, the role of animals and the resulting ASF is mostly limited to converting low-opportunity-cost biomass, thereby avoiding feed-food competition. In future research and communication of more sustainable dietary solutions, these choices and the resulting consequences should be considered to enable effective communication and policy design. Further, we assessed environmental and socio-economic impacts of current dietary choices in Switzerland, and related these to different sociodemographic and lifestyle factors. Our results showed that while nationalities, language regions, age groups, and smoking status seemed particularly distinctive, income or educational groups seemed hardly relevant. Food choices and the resulting sustainability impacts thus markedly differed between these factors, suggesting that these can be relevant, for example, to target nutrition campaigns. Then, we assessed environmental and socio-economic impacts of different consumer strategies for Switzerland, including dietary changes as well as production-side changes. While the dietary changes towards more plant-based diets improved the environmental performance, diet quality, and diet cost, these changes increased social risks. Moreover, increased share of organic agriculture had ambivalent effects on the environmental performance. When enhanced with circularity principles, however, all environmental indicators improved substantially. Consequently, we assessed the nutritional and environmental consequences of integrating animal-source food recommendations with circularity principles, both in national dietary guidelines (Bulgaria, Malta, Netherlands, Sweden, and Switzerland) and for the EAT-Lancet dietary guidelines. While for the latter, ASF protein of the recommendations could be met, we found this not to be feasible for the national guidelines assessed. Nevertheless, considering circularity aspects in dietary guidelines by linking the types of ASF to available low-opportunity-cost biomass offers large potential to improve the resource use efficiency in food systems, and simultaneously improving environmental performance. In conclusion, the role of ASF in more sustainable food systems can be manifold - and depends largely on the required nutritional contributions as well as on defined priorities for different dimensions of sustainability. Importantly, to thoroughly assess the role and potential of ASF for improved sustainability, adequate methods need to be used and adopting a systems-level analysis going beyond product-specific efficiency measures is unavoidable. 



\section{Contents}

Chapter 1 General introduction 1

Chapter 2 Methodological choices drive differences in 9

environmentally-friendly dietary solutions

Chapter $3 \quad$ How food choices link sociodemographic and lifestyle 27

factors with sustainability impacts

Chapter $4 \quad$ Consumer strategies towards a more sustainable

Swiss food system

Chapter 5 Integrating circularity principles with animal-source food

recommendations in national dietary guidelines:

nutritional and environmental consequences

Chapter 6 The compatibility of animal-source food and circularity

in healthy European diets

$\begin{array}{lll}\text { Chapter } 7 & \text { General discussion } & 91\end{array}$

$\begin{array}{lll}\text { Appendix A Supplement to Chapter 2 } & 105\end{array}$

$\begin{array}{lll}\text { Appendix B } & \text { Supplement to Chapter 3 } & 111\end{array}$

$\begin{array}{lll}\text { Appendix C Supplement to Chapter 4 } & 123\end{array}$

$\begin{array}{lll}\text { Appendix D Supplement to Chapter 5 } & 129\end{array}$

$\begin{array}{lll}\text { Appendix E Supplement to Chapter 6 } & 135\end{array}$

$\begin{array}{ll}\text { References } & 139\end{array}$

Summary 151

$\begin{array}{ll}\text { Zusammenfassung } & 154\end{array}$

$\begin{array}{ll}\text { Acknowledgements } & 158\end{array}$

$\begin{array}{ll}\text { About the author } & 160\end{array}$

$\begin{array}{ll}\text { Publications } & 161\end{array}$

$\begin{array}{ll}\text { Education certificate } & 163\end{array}$

$\begin{array}{ll}\text { Colophon } & 166\end{array}$ 

Chapter 1

General introduction 


\section{2 | Chapter I}

\section{Food system sustainability}

Food systems affect both the environment and the human society in many ways (Willett et al., 2019). The production of food causes adverse environmental impacts, ranging from substantial contributions to greenhouse gas (GHG) emissions to land use and associated biodiversity loss (Foley et al., 2011; IPCC, 2019b). Moreover, inputs used for agricultural production substantially altered and still alter the global biogeochemical cycles, leading to nitrogen and phosphorus surplus and consequently eutrophication and acidification (Campbell et al., 2017). At the same time, the production of food is associated with social risks, for example related to labour conditions and human rights (Benoit-Norris et al., 2012).

Next to the societal aspect of eating, food consumption follows the ultimate purpose of providing human bodies with energy and adequate levels of nutrients. Despite this obvious purpose, current dietary habits in many high-income countries fail to achieve a balanced, nutritious diet (Afshin et al., 2019). In these countries, nutrition has shifted towards more processed and refined products, with a higher share of animal-source foods (ASF) (Floud et al., 2011). The link between such suboptimal diets and various non-communicable diseases, for instance diabetes, cardiovascular disease, stroke, and cancer, is well established (Afshin et al., 2019). This development is projected to continue, with dietary habits in emerging economies becoming more similar to dietary habits in high-income countries (Godfray et al., 2018).

The stated areas of concern constitute important dimensions of sustainable food systems. In recent years, food system sustainability was defined in different ways, depending on the underlying set of values and the disciplinary background of the respective authors (Béné et al., 2019). While there are three 'traditional' dimensions of sustainability (environmental, economic, and social), definitions differed as to what these dimensions, in particular the social dimension, encompass. In broad definitions, the social dimension encompasses cultural acceptability as well as human health aspects, which is in line with the Food and Agriculture Organization's definition on sustainable diets stating that diets should be nutritionally adequate, safe, and healthy (Burlingame and Dernini, 2012). In this thesis, I follow recent frameworks for sustainability in food systems that include these aspects in the social dimension (Hebinck et al., 2020; Willett et al., 2019). Hence, the three dimensions environmental, economic, and social (including human health), will be used to define sustainability with regard to food systems in this thesis.

The challenge of sustainably feeding an ever-growing population is addressed with manifold approaches, which range from production- to consumption-side interventions, and combinations thereof. On the production side, such approaches are sustainable intensification or alternative farming practices like the organic production standard (Garnett et al., 2013; Muller et al., 2017). Changes in food consumption of high-income countries mostly target at a reduction of ASF, in combination with healthier plant-source food choices (Theurl et al., 2020; Willett et al., 2019). Overarching interventions are for example circular food systems, where a combination of production- and consumption-side measures is used to effectively redesign current systems towards systems that close biomass and nutrient cycles and account for resource suitability (De Boer and Van 
Ittersum, 2018). In both food consumption and the overarching intervention approaches, reduction of ASF plays a key role (Poore and Nemecek, 2018; Van Zanten et al., 2019; Willett et al., 2019).

\section{Animal-source food in sustainable food systems}

ASF in human diets contributes substantially to the adverse impacts of food systems (Springmann et al., 2018a). According to these authors, the production of ASF contributes around $72-78 \%$ to total GHG emissions of the agricultural sector, and around 20-25\% to pressures on cropland (Springmann et al., 2018a). Next to this environmental burden, consumption of ASF is contested due to animal welfare and general ethical concerns of keeping animals for food production (Verbeke and Viaene, 2000), as well as human health risks, such as zoonotic diseases (Magouras et al., 2020) and antibiotic resistance (Bacanlı and Başaran, 2019).

Furthermore, the ongoing intensification of animal production systems has led to increasing competition between the use of resources for feed and food (Mottet et al., 2017). Feed-food competition can occur in a direct or an indirect way. Direct competition occurs when biomass that is suitable for direct human consumption is fed to animals instead (Wilkinson and Lee, 2018). Indirect competition occurs when feed is cultivated on areas where food for direct human consumption could be cultivated instead (Van Zanten et al., 2016). In direct or indirect ways, biomass is thus allocated to farm animals instead of to humans directly. Independent of how efficiently animals are raised, using these biomass streams for direct human consumption would be more efficient (Foley et al., 2011).

On the upside, animals have the ability to convert low-opportunity-cost biomass (LCB) into valuable ASF, thereby avoiding feed-food competition. LCB includes biomass that is not used for human food consumption, such as by-products from food processing, food waste, and grass resources. Historically, animals were more often fed with LCB (Smil, 2002). However, with increasing intensification of agriculture, animals were bred and fed to be ever more efficient and high-yielding feed converters (Thornton, 2010). Alongside with this development and due to disease risks, the advantage of animals to utilise LCB was exploited less, while feed-food competition increased simultaneously. Recently, this ability, which could define animal's potential future role in more sustainable food systems, has again been discovered as an advantage (Röös et al., 2016; Schader et al., 2015). Limiting animal feed to LCB has subsequently been introduced as one of the key principles of circular food systems (Figure 1) (De Boer and Van Ittersum, 2018; Van Zanten et al., 2019).

With regard to human health, evidence suggests that some ASF can contribute to a healthy and nutritious diet, thanks to its generally high-quality nutritional composition (Adesogan et al., 2020). However, too much of certain types of ASF - in particular red and processed meat - is associated with increased risks for different non-communicable diseases (Afshin et al., 2019; Etemadi et al., 2017). 


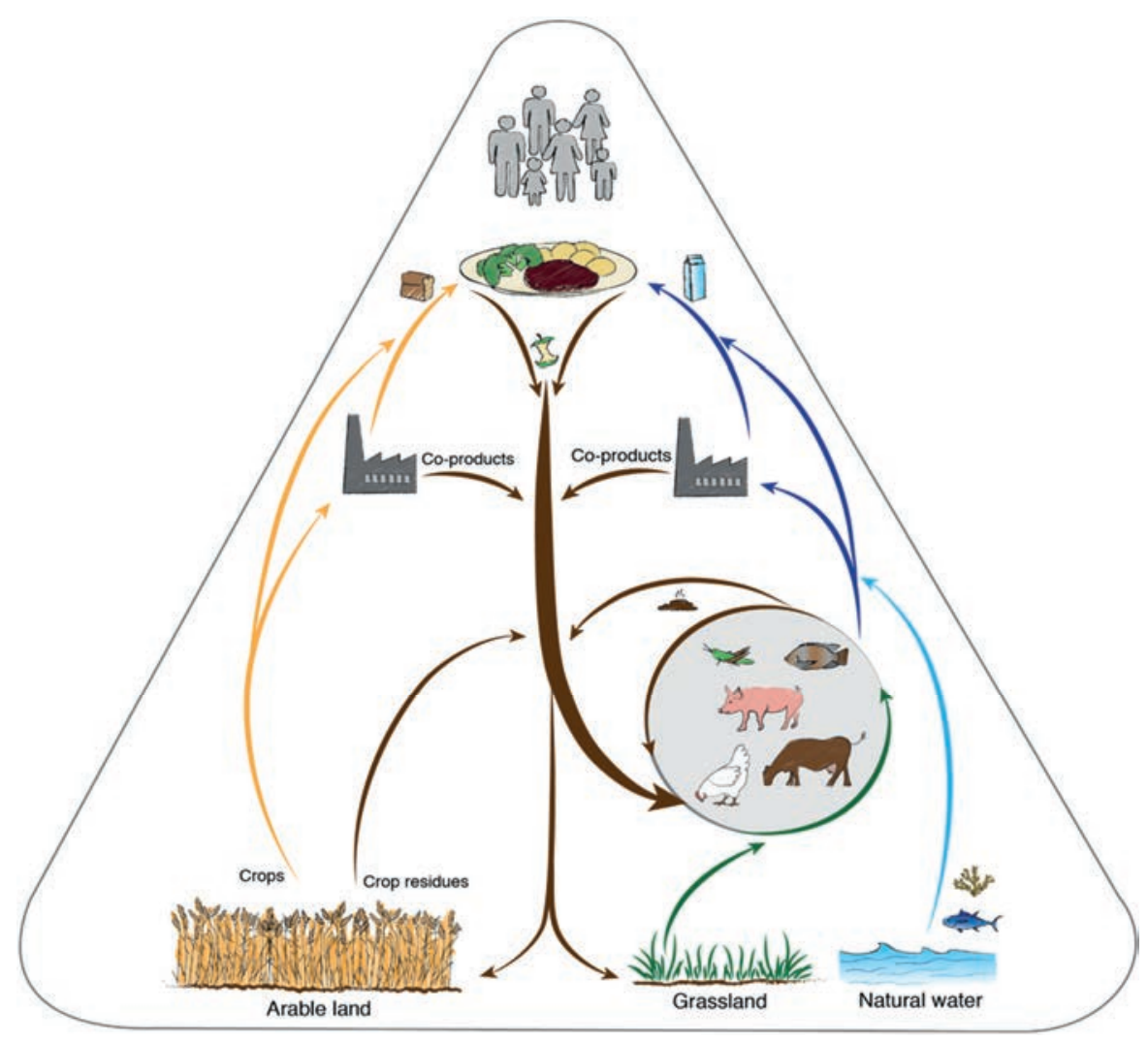

Figure I. Schematic representation of the role of animals in a circular food system (Source: Van Zanten et al. (2019)).

To sum up, the role of ASF in food systems is contested. When assessing strategies to shift towards more sustainable food systems, reducing ASF is predominantly proposed as a solution, from a systems as well as a consumption perspective. How many and which animals could be part of a sustainable food system remains up for discussion, as well as how the remaining ASF should best be produced. This thesis will therefore mostly focus on the role of ASF in sustainable food systems.

\section{Knowledge gaps}

Hence, although much is already known regarding impacts of ASF in food systems, some key knowledge gaps both for current and for potential future food systems with regard to sustainability impacts remain. Of these, four central ones will be addressed in this thesis: 1) identify causes for differences in dietary solutions for ASF among studies, 2) link sociodemographic and lifestyle factors with sustainability impacts of food choices, 3) assess the sustainability performance of different ASFrelated consumer strategies, and 4) assess the compatibility of ASF recommendations in dietary guidelines and circularity principles. To address these knowledge gaps, it is necessary to expand sustainability metrics to adequately cover the various sustainability dimensions within a systemic, and not single-product-focused, context. 


\section{I Differences in dietary solutions}

As stated in the previous section, ASF is at the centre of attention in most dietary solutions that aim to improve the sustainability performance of human diets (Aleksandrowicz et al., 2016; Hallström et al., 2015). There is large consensus that in high-income countries, ASF is currently overconsumed and should hence be reduced (Poore and Nemecek, 2018). However, while this direction is acknowledged, there are conflicting solutions regarding which type of ASF should be reduced to what extent (Van Zanten et al., 2018). For example, while some studies indicate that beef should be reduced most (Abeliotis et al., 2016), other studies suggest that chicken and pork should be reduced more instead (Schader et al., 2015). Understanding the causes that lie behind these differences is key for transparent and effective communication for scientists, but also for policy makers and society in general. Therefore, we investigate the differences in dietary solutions and potential causes that lie behind these differences.

\subsection{Linking sociodemographic and lifestyle factors with sustainability impacts via food choices}

Although frequently-employed dietary scenarios are highly useful to shed light on the option space for more sustainable dietary options and food system states, these scenarios mostly do not reflect realistic dietary choices (Hallström et al., 2015). Macdiarmid et al. (2012) showed that consumers' acceptability substantially limits the potential for climate change mitigation that can be achieved via dietary change, thereby emphasising that this is a key issue to consider in the search for more sustainable diets. Moreover, since food choices differ between population subgroups (Hallström and Börjesson, 2013; Hiza et al., 2013), knowledge on differences in eating habits between subgroups can reveal entry points for targeted nutritional campaigns (Snyder, 2007). To allow for targeted interventions aiming at improved diet quality, for example Pestoni et al. (2019) identified sociodemographic differences in dietary consumptions. However, for the Swiss case, other aspects of sustainability in this regard have so far been less investigated. Therefore, we investigate current dietary habits, their underlying socioeconomic and lifestyle information, and link these to multiple sustainability impacts.

\subsection{Sustainability performance of consumption strategies originating from different production approaches}

Building upon insights from current dietary habits, different strategies to improve the sustainability of these habits emerge. At a consumer level, changing the food composition as well as the type of production of the food products consumed are well-known strategies to improve the sustainability performance of diets (Springmann et al., 2018a; Willett et al., 2019). These strategies are often assessed in dietary scenarios, which can either be predefined or optimised - the latter for example according to specific impact categories, or in order to minimise the difference to current diets (Benvenuti et al., 2019; Ferrari et al., 2020). Recent reviews on dietary changes (Aleksandrowicz et al., 2016; Hallström et al., 2015; Jones et al., 2016; Ridoutt et al., 2017) compiled evidence on the sustainability performance of dietary scenarios, thereby mostly focusing on environmental impacts. While some of these scenarios, such as a vegan diet, are rarely implemented by consumers, others, 


\section{6 | Chapter I}

such as a vegetarian diet, occur more often (Ernstoff et al., 2020). Moreover, also national dietary guidelines constitute often-employed scenarios that link to the respective geographical and cultural circumstances (Gonzalez Fischer and Garnett, 2016; Springmann et al., 2020). All these dietary strategies share a key change in food composition: the reduction of ASF. In addition, by choosing products accordingly, consumers can influence how and where food was produced (Aprile et al., 2012). While labels provide orientation about the production standard employed - for example organic farming - the product's origin needs, in most cases, to be declared as well, thereby providing additional choices for consumers. Although specific sustainability aspects - mostly environmental of some of these strategies have been assessed in a variety of studies (Chen et al., 2019; Springmann et al., 2018a; Von Ow et al., 2020), to date no study has investigated multiple sustainability dimensions of these consumption and production side strategies for the Swiss case study. Therefore, we investigate environmental, economic, and social impacts of different consumer strategies, including dietary changes as well as production-side changes.

\subsection{Compatibility of animal-source food and circularity principles in dietary guidelines}

As stated above, a common starting point for more sustainable dietary solutions are food-based dietary guidelines (FBDG) (Springmann et al., 2020). These are provided at different levels: globally, the World Health Organisation issues recommendations on healthy diets (WHO, 2019), as does the European Food Safety Authority for the European Union (EFSA, 2017). Moreover, currently more than 100 countries issue national dietary guidelines. In general, FBDGs are defined from a diet quality and nutrient requirement perspective. With rising environmental concerns caused by current dietary habits, there is an increased need to consider environmental aspects in the formulation of FBDGs (Tuomisto, 2018).

The fact that national FBDGs capture cultural and geographical circumstances emphasises their importance for site-specific nutrition education and counselling (Brown et al., 2011). A large number of studies has investigated nutritional and environmental consequences of adhering to specific national guidelines (Meier and Christen, 2013; Schwingshackl et al., 2020; Springmann et al., 2020; Van De Kamp et al., 2018). However, these studies operated with fixed environmental impact intensities, thus limiting the option space for ASF to current production practices. Thereby, the potential of limiting animals to convert LCB, thus considering circularity principles, is disregarded. Against this background, further research is required to better understand whether ASF in national FBDGs is compatible with ASF produced on LCB. Therefore, we investigate the nutritional and environmental consequences of integrating ASF recommendations of national FBDGs with circularity principles.

For a broader scope, Willett et al. (2019) recently proposed a healthy reference diet that would allow to stay within the fraction of the planetary boundary allocated to food systems. However, as in the environmental impact assessments of national FBDGs, also these guidelines have been assessed with fixed environmental impact intensities, likewise limiting the role of ASF to current production systems. To what extent this healthy reference diet would be compatible with ASF from circular 
food systems is thus still unknown. Therefore, we investigate the compatibility of the EAT-Lancet healthy reference diet and ASF from food systems that consider circularity principles.

\section{Aim and thesis outline}

This thesis aims to investigate the role of ASF in current and potential future food systems, with special emphasis on integrating different sustainability impacts. To this end, I used different temporal, geographical, and demographic scopes for the assessments (Figure 2). The temporal scope was roughly divided into assessments of current states and of potential future states, the latter including both small and systemic changes. For the geographical scope, I used Switzerland as a main case study country on a national level, which I complemented with four other national European case studies (Bulgaria, Malta, Netherlands, Sweden, in addition to Switzerland), as well as the European Union EU-28 level. Further, with regard to the demographic scope, I conducted two analyses (Chapter 3 and 4) at the level of individual consumers, while Chapters 5 and 6 focused on changes at population level.

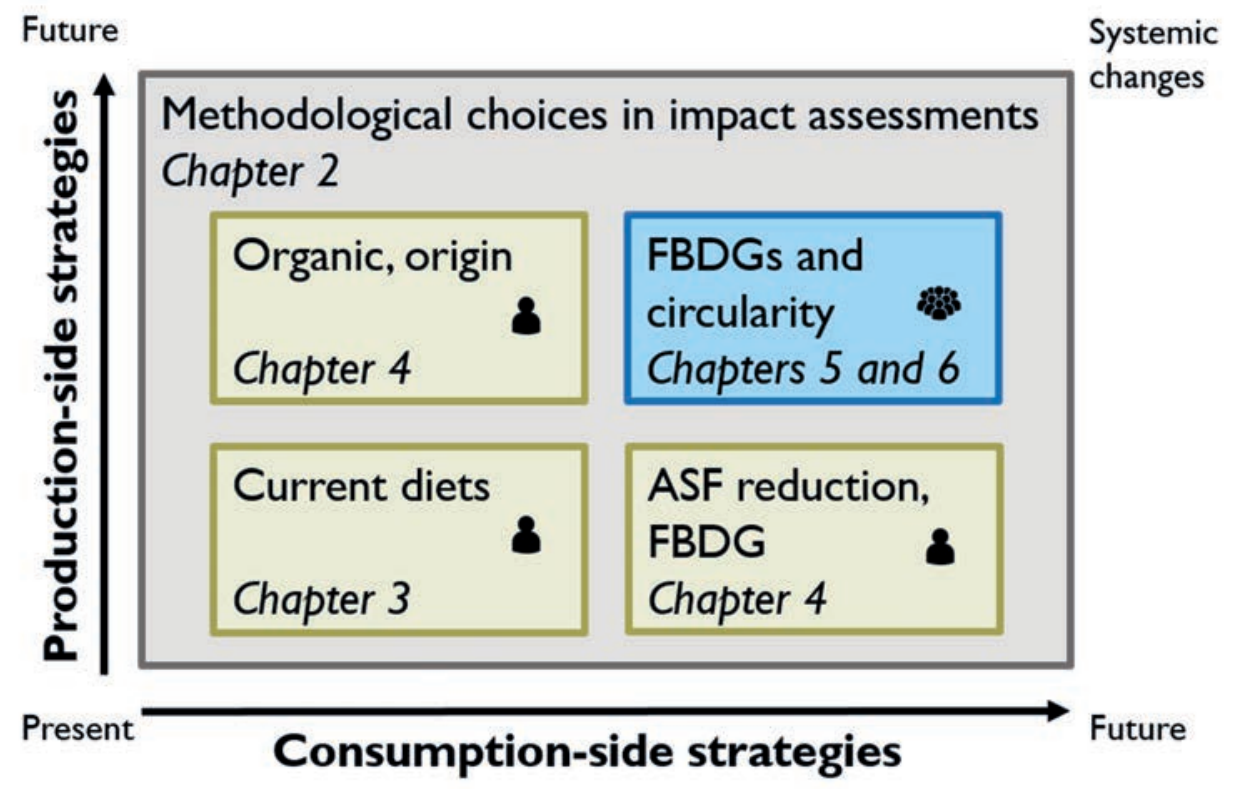

\section{Geographical scope}

\section{Switzerland}

Bulgaria, Malta, Netherlands, Sweden, Switzerland (Chapter 5) / EU (Chapter 6) All

Figure 2. Framework of this thesis, displaying the methodological Chapter 2 in the background and the four more specific Chapters 3-6. ASF: animal-source food. FBDG: food-based dietary guideline. EU: European Union. 


\section{8 | Chapter I}

In order to address the first knowledge gap on differing dietary solutions for ASF, we aimed to investigate in what way these differences can be explained by differences in methodological approaches, leading to Chapter 2. In this chapter, we reviewed scientific literature that assessed more sustainable diets. Based on the outcome of this review, we investigated methodological choices that could be linked to differences in solutions of these diets, and in particular to recommendations regarding ASF.

To address the second knowledge gap, we assessed sustainability impacts of current diets, and how these link to sociodemographic and lifestyle factors in Switzerland (Chapter 3). Moreover, next to environmental indicators, we assessed social risks, diet cost, and diet quality of the current diets. By means of multiple linear regression, we investigated the association between sociodemographic and lifestyle factors and food choices on the one hand, and between sociodemographic and lifestyle factors and sustainability impacts on the other hand. Then, by qualitatively integrating these regression results, we were able to investigate how food choices link sociodemographic and lifestyle factors with sustainability impacts.

After the assessment of current diets, we aimed to assess strategies towards more sustainable food systems that include both consumption- and production-side changes, thereby addressing the third knowledge gap. Thus, in Chapter 4, we assessed environmental, economic, and social aspects of six consumer strategies towards more sustainable food systems for Switzerland. These strategies included dietary changes (meat reduction and following dietary guidelines), but also changes in where and how the provided foods are produced (increased domestic production, organic production, and circularity principles), and, in addition, decreased food waste at consumption stage.

To address the fourth knowledge gap, thus to better understand the compatibility of FBDGs and ASF produced while considering circular food system principles, we investigated national FBDGs (Chapter 5) as well as the dietary guidelines published by the EAT-Lancet commission (Willett et al., 2019) (Chapter 6). In the former, we assessed nutritional and environmental consequences of limiting ASF to the LCB that arises from the plant-source part of five national European FBDGs (Bulgaria, Malta, the Netherlands, Sweden, and Switzerland). By employing scenarios with different nutritional foci, we were able to assess the nutritional option space of ASF based on LCB for national FBDGs. In the latter, we proceeded likewise to derive the LCB from the EAT-Lancet healthy reference diet. Then, we investigated different restrictions on the composition of ASF as well as different levels of plant-based food processing, with implications for the resulting availability of byproducts.

Finally, in Chapter 7, I discuss the implications of the findings for the potential role of ASF in more sustainable food systems. There, I address the identified knowledge gaps with the results of the research performed, and present the overall synthesis of this thesis. 


\title{
Chapter 2
}

\section{Methodological choices drive differences in environmentally-friendly dietary solutions}

\author{
A. Frehner, ${ }^{a, b}$, A. Muller ${ }^{a, c}$, C. Schadera, I.J.M. De Boerb, H.H.E. Van Zanten ${ }^{\text {b }}$ \\ aDepartment of Socioeconomics, Research Institute of Organic Agriculture FiBL, Frick, Switzerland \\ ${ }^{\mathrm{b}}$ Animal Production Systems group, Wageningen University \& Research, Wageningen, the Netherlands \\ Institute of Environmental Decisions, Federal Institutes of Technology Zurich ETHZ, Zurich, Switzerland
}

Global Food Security 24 (2020) 100333 


\section{0 | Chapter 2}

\section{Abstract}

Much scientific literature proposes reduction of animal-source foods to reduce environmental impacts of the food system. However, these dietary solutions differ regarding level and type of animal-source foods. We review this literature and our results show that these differences relate to differences in employed methodological approaches. Approaches that consider systemic consequences throughout the food system propose to limit livestock to low-opportunity-cost feed, where feed availability drives level and type of animal-source foods, resulting in poultry and pork being reduced most. Approaches with fixed impacts propose to reduce animal-source foods depending on environmental intensities, suggesting largest reductions for beef. Thus, by linking differences in dietary solutions to methodological approaches, our results contribute to informed choices of researchers, policy makers, and consumers. 


\section{Introduction}

The current food system - that is all the processes involved in feeding the global human population - connects some of the most pressing environmental challenges of our times. It contributes significantly to approaching or transgressing planetary boundaries (Steffen et al., 2015), such as climate change or the biochemical flow of nitrogen and phosphorus (Campbell et al., 2017). In recent years reducing the environmental impact of the food system has received increasing attention. Next to studies comparing environmental impacts of different production systems (Poore and Nemecek, 2018; Seufert and Ramankutty, 2017), several studies reviewed the environmental impacts of various dietary scenarios (Aleksandrowicz et al., 2016; Hallström et al., 2015; Jones et al., 2016; Ridoutt et al., 2017). Willett et al. (2019) subsequently related environmental impacts of the food system to total resource and emission budgets. They proposed a safe operating space for food systems by defining targets for human diets and for food production. Further, Van Zanten et al. (2018) defined a land boundary for sustainable livestock consumption by reviewing studies that assessed scenarios based on low-opportunity-cost livestock (LOCL) - LOCL describes livestock raised on non-food competing feed $^{1}$, such as food processing by-products, food waste, and grass resources - and comparing them to studies assessing vegetarian or vegan diets.

Recent solutions for keeping the food system within the planetary boundaries often comprise a set of actions targeting both production and consumption aspects. On some dietary solutions, there is agreement among scientists regarding their environmental benefits. This is for example the case for the reduction of animal-source food (ASF) in high-income countries (Poore and Nemecek, 2018; Van Zanten et al., 2018; Willett et al., 2019). However, disagreement arises as soon as these dietary solutions are assessed in more detail, for example regarding which types of ASF should be reduced and by how much (Ridoutt et al., 2017; Schader et al., 2015; Van Zanten et al., 2018). Disagreement also prevails with regard to how production systems should look like (Muller et al., 2017; Poore and Nemecek, 2018). While some studies conclude that ASF from monogastric animals (mainly pork and poultry) should be favoured over ruminants, others argue that grassland-based ruminant production can contribute to food security by avoiding competition with resources that could be used for direct human food production. Understanding why these differences in dietary solutions for sustainable food systems and diets occur is highly relevant, as such dietary solutions based on scientific results serve as evidence base for environmentally-oriented dietary guidelines as well as policy making.

Therefore, we aim to investigate in which way these differences in dietary solutions can be explained by differences in methodological approaches. To this end, we reviewed scientific studies that compared environmental impacts of human diets in order to identify conformity as well as differences in dietary solutions. Further, choices that need to be taken to specify the type of dietary scenario and the modelling approach used to assess the environmental impact of human diets, hereafter denoted by approach-related choices, are assessed within the reviewed studies. These

\footnotetext{
${ }^{1}$ Also referred to as low-opportunity-cost biomass in this thesis.
} 


\section{I2 | Chapter 2}

approach-related choices are then grouped to typical methodological approaches and related to dietary solutions in order to understand why different dietary solutions occurred.

\section{Material and methods}

We conducted a review of scientific literature to investigate how approach-related choices affect the differences in dietary solutions derived from assessments of the environmental performance of different diets. We applied keyword search, Boolean operators, and screened references of recent review articles to identify relevant articles. More information on the review approach can be found in Appendix A1.

Following the literature search, all articles were scanned and qualitative information on choices that are required in the process of calculating environmental impacts of dietary scenarios were collected. These approach-related choices were first deduced from the life cycle assessment (LCA) framework, as LCA was the most commonly used method to calculate environmental impacts in the papers reviewed (37 out of 56 studies). Seven choices are directly deduced from the LCA framework: definition of the 1) system boundary, 2) functional unit, 3) impact categories, 4) allocation, 5) inventory analysis, 6) impact assessment, and 7) treatment of consequences. Further, as in dietary scenarios also absolute restrictions on resource availability and on sink capacities become important, an additional approach-related choice was identified: 8) restrictions on resource availability and on sink capacities for emissions. Based on the qualitative information collected for the approach-related choices, the possible values for each choice were defined (thus, e.g. for the approach-related choice system boundary, the values are 'cradle-to-farm-gate', 'cradle-to-distribution', etc.; see Frehner et al. (2019)). In a next step, all studies were reviewed a second time, where the defined values of each approach-related choice were assessed (Frehner et al., 2019). Then, based on prevailing combinations of values of several approach-related choices, typical methodological approaches for scenario specification and environmental impact assessment were identified.

The dietary solutions in the studies were compiled as follows: on the one hand, dietary scenarios were quantitatively assessed, by extracting the consumed amount per food group per person. On the other hand, reduction of ASF was specifically assessed as follows: first, reduction solutions per ASF category were identified. Second, the suggested optimal range per ASF was classified as follows: as low as possible, intake based on LOCL, or a (nationally) recommended level. Third, the solutions for substitutes to compensate the reduction in nutrient intake were assessed.

Finally, congruent as well as differing dietary solutions among methodological approaches were identified, and their implications for possible food system states were discussed.

\section{Results}

We first describe general characteristics of reviewed studies. Subsequently, we introduce the approach-related choices and present results on their values as utilised in the reviewed studies. Following this, typical methodological approaches are proposed, and the dietary solutions for reducing the environmental impact of the food system presented. Then, congruent and differing dietary solutions are identified and related to typical methodological approaches. 


\section{I General characteristics}

In total, 56 studies were identified that fulfil all inclusion criteria (Frehner et al., 2019). The geographical scope of these studies varied; 32 performed a national, 11 a regional, and 13 a global assessment. Further, more than half of the studies $(n=33)$ performed an assessment for current circumstances, whereas 23 studies employed a future temporal scope.

\subsection{Approach-related choices}

1) System boundary

System boundaries describe the boundaries of the system studied; thus, the main focus is on which stages of the production process are included. In a wider context, also temporal and geographical scope contribute to the system boundaries. The studies covered the production stages as follows: 16 studies considered impacts from cradle-to-farm gate, 8 from cradle-to-distribution/retail, 15 from cradle-to-consumer, and 10 from cradle-to-grave. For 7 studies the system boundaries could not be detected in the description of the methods, or differing system boundaries were used for different food groups.

2) Functional unit

In the studies reviewed, the functional unit assessed is rarely mentioned explicitly. Implicitly, the functional unit can be derived from the temporal scope and the fraction of the population assessed. Different diets are thus compared on the basis of nutritional value per part of the population per time frame (e.g. kcal/person/day).

3) Impact categories

Resulting from our inclusion criteria, all studies assessed greenhouse gas (GHG) emissions ( $\mathrm{n}=49$ ) and/or land use (n=35). Further, 10 studies include indicators related to nitrogen and/or phosphorus surplus, 6 assess eutrophication and/or acidification, and 7 assess fossil energy use. Only 3 studies employ an indicator related to biodiversity (biodiversity damage potential $(n=2)$ and extinction rate $(n=1))$.

\section{4) Allocation}

When a process has multiple outputs, the total resulting environmental impact needs to be allocated to each individual output. According to the ISO-guidelines (Finkbeiner et al., 2006), allocation should be avoided whenever possible, by increasing the level of detail or using system expansion. If it is unavoidable, physical or economic relations should be employed to allocate.

For the assessment of diets, it is not only relevant which allocation method is applied, but also how co-products, i.e. products that result from the same process (such as milk and ruminant meat), are treated in the assessment after allocation has taken place. Two approaches are prevailing: first, to follow a product, and second, to follow nutrients. Approaches that follow products allocate the total resulting environmental impact based on the chosen allocation method (mostly economic allocation). Then, products that are not of primary interest are allocated out of the system boundary of the study; thus, they are allocated a share of the environmental impact, but are not included in 


\section{I4 | Chapter 2}

the subsequent analysis. An example for this would be a vegetarian scenario, where human diets contain milk produced by ruminants, but the associated meat is not considered in the consumption. Even though the environmental impact of dairy farming is also partly allocated to the associated meat, it is not included in the total environmental impact of a vegetarian diet, and is thus allocated out of the system. In literature, approaches that follow products mainly base their environmental impacts upon factors from single product LCAs $(n=41)$. Approaches that follow nutrients, however, steer their analysis not on product, but on nutrient level, and therefore, after allocation, trace nutrients and thus keep all products with relevant nutrients in the system. Following nutrients complicates the modelling, as links between co-products have to be considered throughout the assessment. Following nutrients $(n=15)$ is employed for example in mass- and nutrient-flow models.

5) Inventory analysis

In the inventory analysis, resource use and emissions to air, water, and soil of all inputs and outputs of processes that fall into the previously defined system boundary are inventoried. Thus, inventory analysis refers to the data that are used as basis for the calculation of environmental impacts. Here, the approaches reviewed differ in their starting level, i.e. whether they conduct a bottom-up or a top-down inventory.

On the one hand, we identified studies that conducted a bottom-up inventory $(n=43)$, using a product level approach. Then, by assuming a linear relationship, the product level inventories are scaled up to the level of the functional unit (e.g. inventory made for $1 \mathrm{~kg}$ of carrots; then, if the assessment is done for the population of Germany, the inventory for $1 \mathrm{~kg}$ of carrots is scaled up to the amount that is consumed in the diet of the German population). On the other hand, studies that conduct a top-down inventory analysis $(n=13)$ start from aggregated values and allocate based on nutrient requirements, for example (thus, they take e.g. the total emissions attributed to fertilizer application on agricultural areas in a certain region and distribute them to the single crop products based on the relative nutrient requirements of the different crops).

6) Impact assessment

Impact assessment is conducted by applying characterisation factors to the emission and resource inventories. Thus, for e.g. GHG emissions, factors are applied to bring all different GHGs to a common unit. These commensurable values are then added up. For GHG emissions, global warming potential over a time frame of 100 years is most often used, but there is some discussion on whether or not this is the most appropriate measure, in particular for methane which shows considerably different warming dynamics over time than $\mathrm{CO}_{2}$ (Persson et al., 2015).

\section{7) Consequences}

When assessing diets on a national, regional or global level, changes in diets are likely to cause a cascade of changes in production systems and trade patterns. As a result, the environmental impacts per reference unit may change as well. In the modelling process, one has to decide how such consequences of changes on environmental impacts are treated. One possibility - which is abundant in literature $(n=38)$, is to ignore the consequences and thus keep environmental impacts per reference unit constant (mostly by using attributional LCA factors). Another approach is to follow 
consequences of changes for single products by applying consequential LCA $(n=2)$. The third option how to deal with consequences is to apply a modelling approach that captures the interlinkages between relevant processes and calculates the environmental impacts per reference unit endogenously $(n=16)$. By this, it is possible to trace the resulting consequences. However, such an approach requires more modelling complexity, which then might require simplifications in other aspects (e.g. regarding the level of detail in crop coverage). Examples for such modelling approaches are biophysical models (e.g. (Bajželj et al., 2014; Erb et al., 2016; Muller et al., 2017)) or material flow analysis (Thaler et al., 2015).

8) Restrictions on resource availability and on sink capacities for emissions

Absolute restrictions on resource availability and on sink capacities for emissions and the resulting scarcities (called 'restrictions on resource use and sink capacities' in the following) become relevant when diets are assessed for a broader spatial scope. Thereby, restricted resource availability refers to water scarcity or availability of land of a certain quality, for example, while the limitations on sink capacities become relevant in the context of carrying capacities of ecosystems, e.g. for nutrient inflows. However, estimating the contributions of the food system for a broader spatial scope and therein also in relation to other activities and how to include those in the assessment can be complex. Taking the example of land use, land quality differs drastically between regions, which implies differing comparative advantages for different crop and livestock varieties (Zabel et al., 2014). Feed produced on arable land competes with direct crop production for humans, while feed sourced from grassland unsuitable for human food production can contribute to food security by allowing to use additional area and associated biomass for the production of human-edible ASF. Of the studies reviewed, 17 consider resource scarcities and limited sink capacities in relation to the food system in their assessment, while 39 do not.

\subsection{Typical methodological approaches}

The approach-related choices identified above potentially affect the outcome of the studies regarding solutions for environmentally-friendly diets. Thereby, we can discern two broad areas where the choices can become relevant, either via the way how scenarios are specified (not to be confused with specific scenario assumptions, e.g. the level of bioenergy use), or via the way how the environmental impact assessment is undertaken. Based on prevailing combinations of values of approach-related choices in the studies reviewed, typical methodological approaches for scenario specification and for environmental impact assessment were identified, see Table 1. These typical methodological approaches can be applied to most of the studies, but not all; for scenario specification, four studies do not fit into the proposed typical methodological approaches, and for the environmental impact assessment, seven studies (see Appendix A2). We will first present the case of the approach-related choices regarding functional unit, impact categories, allocation, and restrictions on resource use and sink capacities that become effective via the way how scenarios are specified. Subsequently, we present the case of the choices regarding allocation, inventory analysis, consequences, and restrictions on resource use and sink capacities that become effective via the environmental impact assessment. Choices regarding system boundaries and impact assessment are not further pursued, due to a lack of indication to affect the outcome of the studies. 


\section{6 | Chapter 2}

Table I. Approach-related choices that are considered for the definition of typical methodological approaches on scenario specification and environmental impact assessment (in italics: values per approach-related choice).

\begin{tabular}{|c|c|c|c|c|}
\hline \multirow[t]{2}{*}{ Approach-related choices } & \multicolumn{2}{|c|}{$\begin{array}{l}\text { Typical methodological } \\
\text { approaches: scenario } \\
\text { specification }\end{array}$} & \multicolumn{2}{|c|}{$\begin{array}{l}\text { Typical methodological } \\
\text { approaches: environmental } \\
\text { impact assessment }\end{array}$} \\
\hline & $\begin{array}{l}\text { Consumption- } \\
\text { oriented }\end{array}$ & $\begin{array}{l}\text { Resource- } \\
\text { oriented }\end{array}$ & $\begin{array}{l}\text { Fixed impact } \\
\text { assessment } \\
\text { (FIA) }\end{array}$ & $\begin{array}{c}\text { Systemic } \\
\text { consequences } \\
\text { analysis (SCA) }\end{array}$ \\
\hline \multicolumn{5}{|l|}{ System boundary } \\
\hline Functional unit & $x$ & $x$ & & \\
\hline \multicolumn{5}{|l|}{ Allocation } \\
\hline Follow products & $x$ & & $x$ & \\
\hline Follow nutrients & & $x$ & & $x$ \\
\hline \multicolumn{5}{|l|}{ Inventory analysis } \\
\hline Product level & & & $x$ & \\
\hline Systems level & & & & $x$ \\
\hline \multicolumn{5}{|l|}{ Impact assessment } \\
\hline \multicolumn{5}{|l|}{ Consequences } \\
\hline No & & & $x$ & \\
\hline Yes & & & & $x$ \\
\hline \multicolumn{5}{|l|}{$\begin{array}{l}\text { Restrictions on resource use } \\
\text { and sink capacities }\end{array}$} \\
\hline No & $x$ & & $x$ & \\
\hline Yes & & $x$ & & $X$ \\
\hline
\end{tabular}

\subsection{Scenario specification}

The choices regarding functional unit and impact categories affect scenario specification in a general way, and are thus explored first. Then, two typical approaches for the specification of scenarios, based on the choices regarding allocation and restrictions on resource use and sink capacities, are proposed: resource-oriented scenario specification and consumption-oriented scenario specification.

The functional unit employed differs between studies, but can also differ within studies, by varying amounts of foods and thus micro- and macronutrients. In scenarios that are not isocaloric, effects of changes in dietary patterns are mixed with total reductions or increases in caloric intake, and thus cannot be straightforwardly interpreted anymore. Examples for this are comparisons of dietary patterns that follow dietary guidelines $(n=16)$ - and accordingly limit caloric intake to the recommended levels - with current diets, where the caloric intake exceeds the recommended levels. Another example is food waste reduction $(n=18)$; in scenarios that assume less food waste along the 
production chain, less food has to be produced to provide the same amount of food for human consumption, and thus, also the absolute environmental impacts are scaled. When interpreting results from studies assessing scenarios that include different amounts of caloric intake or different food waste reduction rates, the effects of changes in dietary compositions need to be interpreted carefully and disentangled from effects resulting from differing caloric levels. To facilitate interpretation, the impact of dietary composition can be isolated by normalising the caloric content of the modelled dietary scenarios. Next to the functional unit, impact categories can affect scenario specification, if scenarios are defined based on performance of specific impact categories. This is the case in studies that employ diet optimisation (Gazan et al., 2018), where dietary scenarios are derived endogenously based on constraints on, for example, GHG emissions (e.g. (Donati et al., 2016)).

Further, how allocation is treated (i.e. whether co-products are allocated out of the system, thus products are followed, or whether nutrients are followed) can influence the dietary composition in scenarios; if nutrients are followed, consistent proportions between co-products are used in the diets. On the contrary, if co-products are allocated out of the chosen system boundary, dietary compositions that are inconsistent from a production perspective can result. Furthermore, how restrictions on resource use and sink capacities are treated affects the dietary composition. If biomass resources are treated differently depending on suitability for human consumption, production systems that focus on animals that are able to convert biomass streams unsuitable for human consumption into human-edible foods perform better than those that cannot. Examples for this are ruminants raised on grassland unsuitable for arable farming and pork raised on food waste (Van Zanten et al., 2018). On the other hand, when suitability for human consumption is not considered, ASF is included in the diet according to the lowest intensities (e.g. land or GHG emissions) per $\mathrm{kg}$ of output; then, intensive grain-fed poultry systems perform better compared to grass-fed beef cattle, as the total land used for the production is lower (Van Zanten et al., 2018). Thus, in the latter case, competition for arable land between feed and food cannot be disclosed, which poses an important limitation and needs to be considered when interpreting such results.

Resource-oriented scenario specification $(n=14)$, on the one hand, is hence characterised by following nutrients in the allocation and considering restrictions on resource use and sink capacities in the specification. Thus, production restrictions, such as co-product links and scarcity of resources, are taken into account, and the suitability of resources is considered in the production systems and dietary composition. Consumption-oriented scenario specification $(n=38)$, on the other hand, is characterised by following products in the allocation, and absolute restrictions on resource use and sink capacities are not specifically related to in the framing of the scenario. This can then lead to scenarios that are inconsistent from a production perspective (see example on vegetarian diets in Section 3.2).

\subsubsection{Environmental impact assessment}

Two typical approaches for environmental impact assessment can be defined according to how the identified choices allocation, inventory analysis, consequences, and restrictions on resource use and sink capacities are treated: fixed impact assessment (FIA) and systemic consequences analysis (SCA). 
FIA is characterised by applying fixed impact factors per food product when assessing dietary scenarios. More precisely, studies applying FIA $(n=36)$ follow products in the allocation - thus, coproducts are allocated out of the system. Further, inventory analysis is done on product level, and then, linear scaling is applied to reach the level of interest (i.e. a diet for a certain part of the population). Consequences are not considered in the assessment. Lastly, restrictions on resource use and sink capacities are not considered according to suitability (e.g. land). In contrast to this, studies performing a SCA $(n=13)$ consider systemic changes and boundaries in the modelling, and by this, typically generate impact factors per product within a consistent food system state endogenously. For the identified choices, this can be depicted as follows: allocation is treated such that nutrients are followed and all co-products are thus kept in the system. Further, inventory analysis is often done on an aggregate level, and inputs, such as fertiliser, are e.g. allocated based on relative nutrient requirements of different crops. Consequences are included by considering level-induced changes for production systems (i.e. assuming that the way how products can be produced depends on how much is produced, for example limiting ASF to non-food competing feed) and consequential implications for environmental impacts per unit output. Alongside with this, restrictions on resource use and sink capacities are captured where relevant.

\subsection{Solutions for reducing environmental impacts of the food system}

In the following, the solutions of reviewed studies regarding changes in human diets and production-side measures are presented, and congruent as well as differing solutions between studies are identified.

\subsubsection{Changes in human diets}

Solutions for changes in human diets point at food groups, i.e. ASF and plant-source food, and absolute reduction of food intake until caloric intake is at the recommended level (solutions for studies not falling into the proposed typical methodological approaches can be found in Appendix A2). Studies agree that in high-income countries, ASF needs to be reduced; more precisely, in Figure 1 we see that all studies agree on a reduction of pork and beef, while for a reduction of poultry, the majority of studies agrees. To a lesser extent also reductions of dairy, eggs, and fish and seafood are proposed. However, which ASF should be reduced most and how the remaining (if any) ASF should be produced, remains inconclusive.

Studies following a consumption-oriented scenario specification and fixed impact assessment either suggest to reduce beef, pork and poultry as low as possible, or they propose to keep a certain level of ASF as part of a recommended healthy diet (Figure 1). Studies employing a resource-oriented scenario specification and systemic consequences analysis mostly propose a reduction of all ASF to the level that can be sustained based on LOCL. In LOCL scenarios, resulting amounts per capita per day for beef range between 10 and $55 \mathrm{~g}$, for pork between 7 and $46 \mathrm{~g}$, and for poultry, between 0 and 26 g per person per day.

Another point for discussion is how to substitute for the reduction in ASF - be it by other types of ASF, such as dairy, or by plant-based protein sources, such as legumes. Figure 2 shows the proposed 
food groups per methodological approach. All studies agree to take plant-source food as substitute for the reduction in ASF. Further, some studies employing consumption-oriented scenario specification and FIA also include dairy, fish and seafood, and to a lower extend eggs, poultry, and pork as possible substitutes.

Regarding land use, in Figure 3, relative land use (differentiated by land type) per additional g of ASF-based protein in the human diet is presented. For values below $30 \mathrm{~g}$ of ASF-based protein, there is no clear trend as to which level of ASF and which products perform best regarding total land use. For cropland only and studies employing SCA, these results look different; two of these studies found that less cropland is required if some livestock, based on the LOCL principle, remains in the system, compared to a vegan scenario (Röös et al., 2017; Van Kernebeek et al., 2016). In contrast to this, studies operating with FIA find increased land use for all land types with increased levels of ASF in the human diet. For values above $30 \mathrm{~g}$ of ASF-based protein, both SCA and FIA studies find increased land use.

For GHG emissions (see Figure 3), the results are clearer, meaning that according to currently available estimates, scenarios with less ASF generally perform better. Also for LOCL scenarios, one study finds that GHG emissions are higher than for purely plant-based diets (Röös et al. (2017)).

To sum up, when looking at total land use and GHG emissions, scenarios with ASF as low as possible - down to vegan, in the most extreme case - perform best. When looking at cropland use only, scenarios with ASF from LOCL perform best according to SCA studies, while FIA studies do not find different results compared to total land use. 


\section{$20 \mid$ Chapter 2}

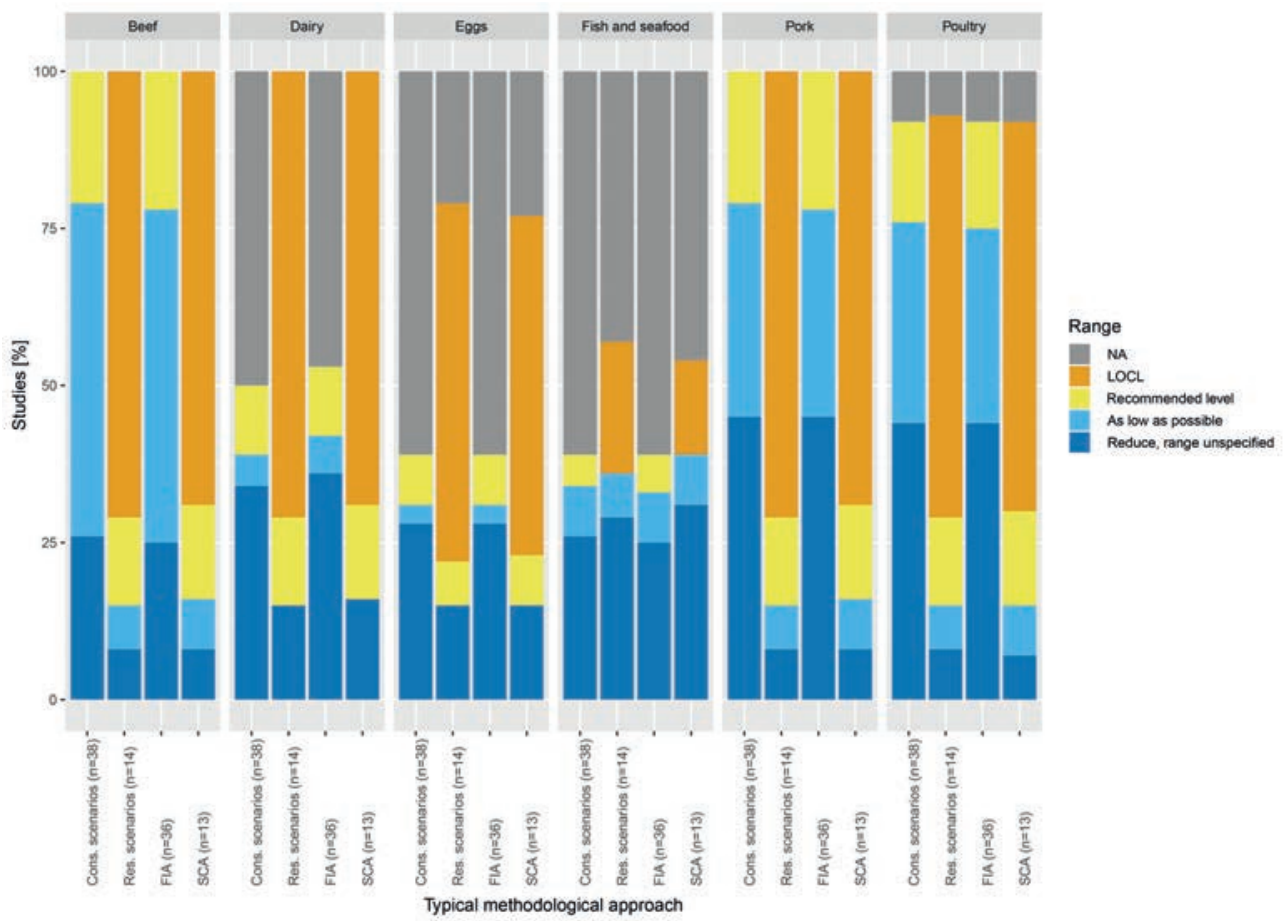

Figure I. Inventory of optimal ranges for animal-source food reduction solutions to achieve reduced environmental impacts of the food system (\% of the studies per typical methodological approach; consumption-oriented scenario specification (Cons. Scenarios), resource-oriented scenario specification (Res. Scenarios), fixed impact assessment (FIA), and systemic consequences analysis $(\mathrm{SCA})$ ). Colours indicate the proposed range; low-opportunity-cost livestock (LOCL), recommended level, as low as possible, reduce with unspecified range, and not assessed (NA). 


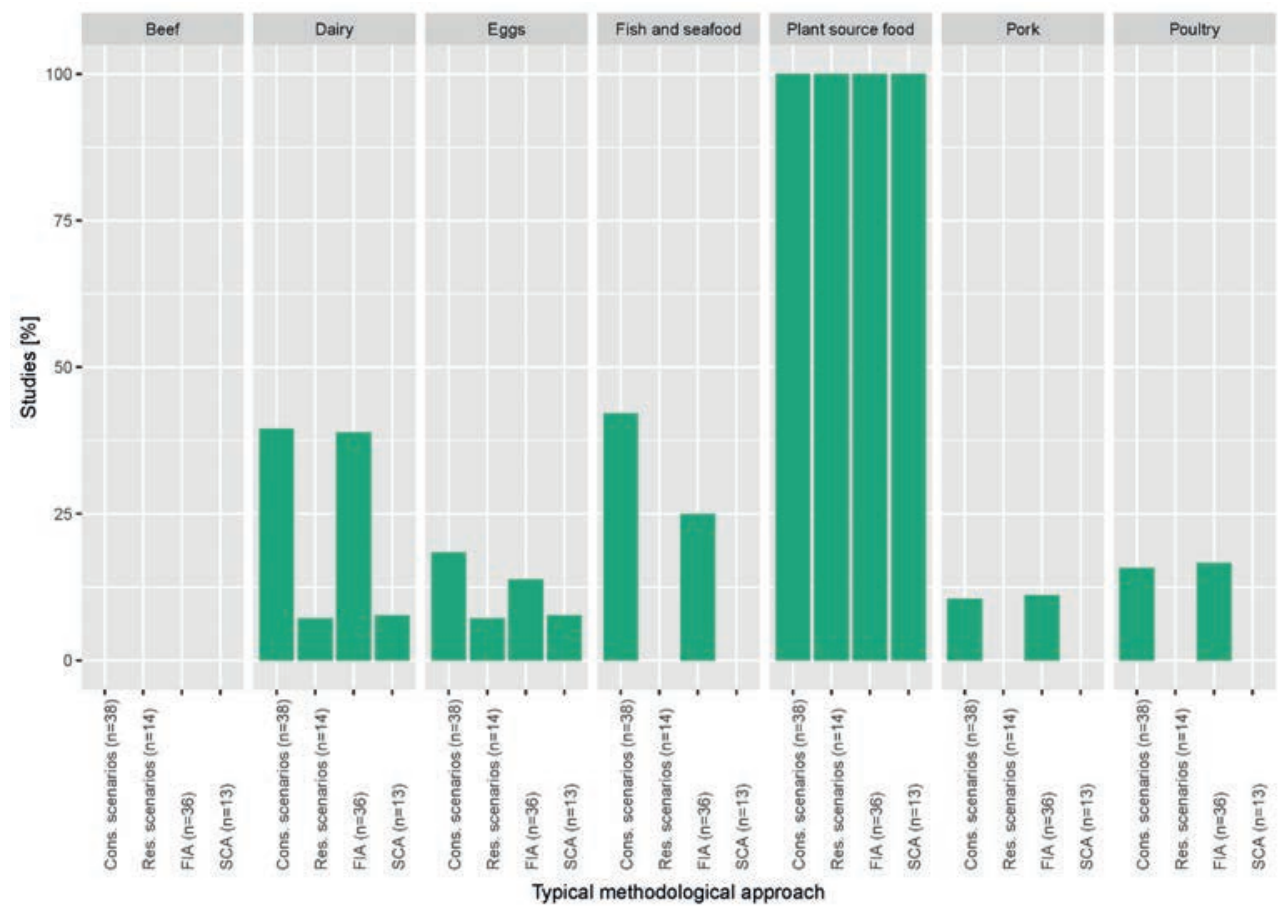

Figure 2. Inventory of substitution solutions (\% of the studies per typical methodological approach; consumption-oriented scenario specification (Cons. Scenarios), resource-oriented scenario specification (Res. Scenarios), fixed impact assessment (FIA), and systemic consequences analysis (SCA)).
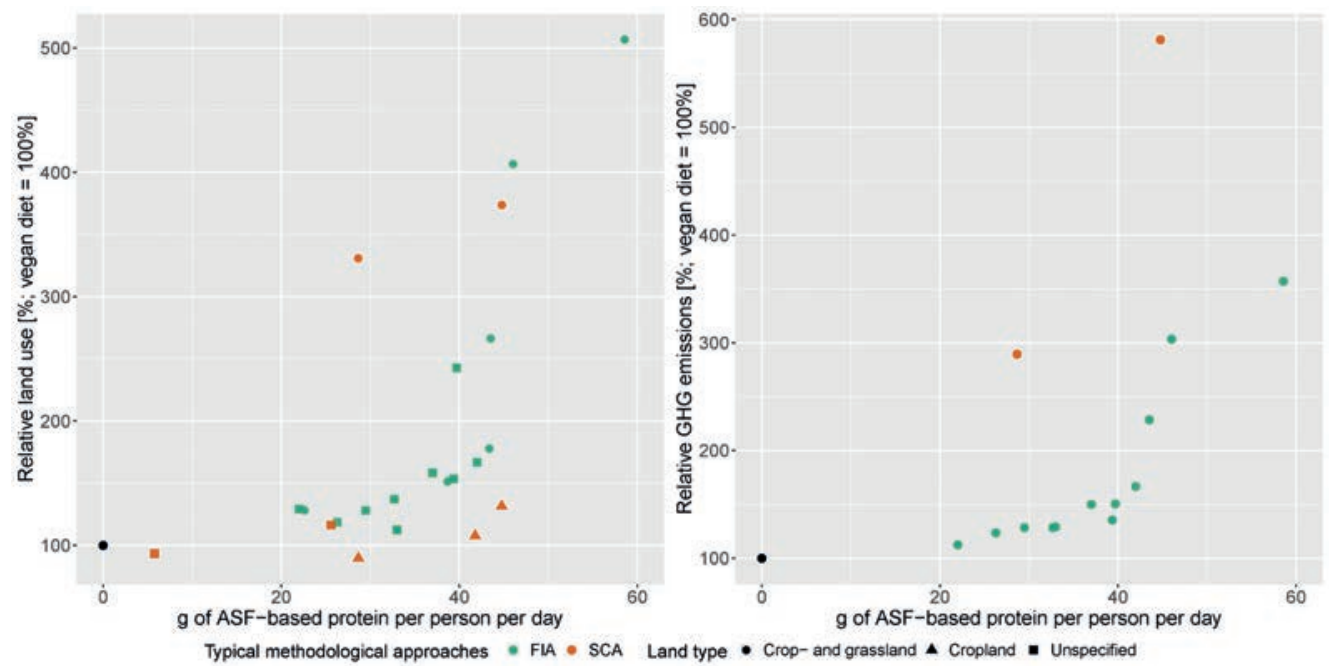

Figure 3. Left: relative land use per $\mathrm{g}$ of animal-source food (ASF)-based protein per person per day (land type is indicated by shape). Right: relative greenhouse gas (GHG) emissions per g of ASF-based protein per person per day. Each dot represents one dietary scenario. Colours indicate typical methodological approaches; fixed impact assessment (FIA) and systemic consequences analysis (SCA). Land use and GHG emissions of dietary scenarios are presented relative to vegan scenarios of the same studies (black dot) in percentage. Caloric consumption across scenarios is normalised to $2000 \mathrm{kcal}$ to facilitate comparison. 


\section{2 | Chapter 2}

\subsubsection{Changes in production systems}

Generally, solutions identified in the studies reviewed focus on changes in food consumption, resulting from our inclusion criteria. Next to these solutions, some studies also include different production systems in their assessment, leading to additional solutions regarding how the recommended foods should be produced. Such changes in food production systems can for instance be to follow existing trends, such as sustainable intensification $(n=10)$ and closing yield gaps $(n=6)$. Another possibility is to include well-defined production standards, such as organic farming $(n=8)$. A third option is to highlight single management options in the production process (which however can require substantial changes in the system), regarding animal production $(n=16)$, or plant production $(n=12)$. Management options for animal production systems often focus on feed that avoids competition with direct food production $(n=11)$, previously defined as LOCL (Van Zanten et al., 2018), or then, to reduce feed conversion ratio $(n=3)$. Specific options named to improve plant production are e.g. crop rotations. Lastly, 18 studies assess the effects of a reduction in food waste, which takes effect on all actors along the value chain.

Although diverse changes in production systems are included in several scenarios, mainly scenarios based on LOCL propose consistent large-scale changes of food production linked with food consumption. These studies propose to limit animal numbers to the amount that can be sustained based on the local productive capacity, i.e. that ruminants should mainly be raised on available grasslands (with or without temporary meadows as part of crop rotations). Further, the recommended levels for pork, poultry, and eggs are mostly based on locally available by-products and, in some studies, on food waste. The optimal mix and allocation of low-opportunity-cost feedstuff to different livestock systems is subject of current research (Van Hal et al., 2019a).

In conclusion, all studies agree on a reduction for ASF for the geographical scope considered in this review. Further, studies that employ a consumption-oriented scenario specification and FIA either recommend a maximum reduction of ASF, or if some ASF remains, this should mainly be sourced from dairy, eggs, fish and seafood, pork, and poultry. For these, often, no specific recommendation with regard to production systems is given. On the other hand, studies that employ a resourceoriented scenario specification and SCA mostly recommend a remaining low level of ASF based on the LOCL principle, and thus come with clearer solutions how this remaining ASF should be produced. Additionally, the solutions in studies that do not fit into the identified typical methodological approaches for scenario specification resemble those of the consumption-oriented scenario specification, and solutions in those that do not fit in the typical methodological approaches on environmental impact assessment, are mixed, thus, could be part of both FIA and SCA.

\section{Discussion}

\section{I Suitability of typical methodological approaches}

Generally, the methodological approach should be chosen according to the aim of the study. With regard to typical methodological approaches on scenario specification, we can conclude that consumption-oriented scenarios are suitable for small-scale changes, where either the geographical 
scope is restricted, or only part of the population is assessed. For large-scale changes, and especially global assessments, resource-oriented scenarios are suitable, as they include consistent proportions between products that originate from the same production process.

For the environmental impact assessment of dietary and associated food system change, we identified two typical methodological approaches: FIA and SCA. On the one hand, FIA is characterised by using status-quo intensities, and therefore, this approach is well suited to assess small-scale dietary changes for the current situation. On the other hand, SCA considers systemic consequences of dietary changes on processes in the system and resulting environmental intensities; therefore, SCA is well suited to assess large-scale dietary changes for the future situation.

\subsection{Sustainability strategies}

Results from identified methodological approaches have several implications for how environmentally sustainable food systems would look like. On the one hand, when consumptionoriented scenario specification and FIA are applied, major changes of food consumption are proposed; either a maximum reduction of ASF, or small amounts of ASF that are mainly sourced from dairy, eggs, fish and seafood, pork, and poultry. However, on the production side, often no changes are assumed, and thus, also the environmental impacts per unit output stay constant. If changes in production systems are assumed, then sometimes in form of efficiency increases and thus higher yields per area or per animal head. Relating this to sustainability strategies on food system level (Huber, 2000; Schader et al., 2014), with such a system, two sustainability strategies are covered; efficiency and sufficiency. Efficiency by altering the diet towards products exhibiting lower impacts per unit output, or by assuming efficiency increases in the production chain. Sufficiency is covered as the dietary change assumed requires behavioural shifts that change people's choices towards reduced ASF.

On the other hand, in studies that apply SCA, define scenarios from a resource perspective, and conclude that a low amount of ASF remaining in the food system should be based on LOCL, the resulting food system state does not only assume changes on the consumption side, but also proposes a coherent change of the production system. With this, the sustainability strategies efficiency, sufficiency and consistency can be covered. Efficiency however is not covered per product (i.e. lower impacts per amount of inputs), but on food system level, by accounting for efficient resource use for the whole system. An example for this is that ASF produced with non-food competing feed might be less efficient from an impacts - inputs perspective, since animals have to cope with suboptimal diets and therefore might not be able to follow optimal growth rates (Van Zanten et al., 2019). However, from a food systems perspective, such a feeding regime can be even more resource efficient, because feed that cannot be used for direct human consumption is used to produce human-edible products. The same argument can be used to explain why the consistency strategy is covered; the resulting system links to concepts such as optimal resource use from a systemic perspective, related to closed and circular nutrient flows and waste reduction (hence the focus on utilising by-products for feed, for example). 


\section{4 | Chapter 2}

\subsection{Other environmental impacts}

Next to GHG emissions and land use, the production of our food causes other adverse impacts on the environment, such as biodiversity loss, eutrophication, pollution, and water scarcity. Due to lack of adequate evidence across reviewed studies, these impacts were not included in the present analysis. Nevertheless, considering these impacts is important and future research should focus on improving quantification and standardisation of these impacts. Then, a more complete view on the environmental impacts of dietary scenarios could be obtained.

\section{Conclusion}

We draw conclusions for three target groups: researchers, policy makers, and civil society.

First, for researchers, we note that in studies assessing future food system states resulting from dietary change, the modelling approach used should be able to capture the resulting consequences. Large-scale dietary changes will cause a cascade of effects, and require cautious consideration of coproduct links and suitability and scarcity of resources. To assess such changes and propose suitable solutions, systemic consequences analysis (SCA) studies are adequate, while fixed impact assessment (FIA) studies are not able to capture the full consequences of such changes. FIA is however adequate to assess the impacts of the current food system or of relative small systems, where changes have negligible effects only beyond narrowly chosen boundaries.

Second, solutions from the two typical methodological approaches result in different implications for policy makers. Results from studies applying a consumption-oriented scenario specification and FIA could generally be implemented with policies targeting at consumption-side and efficiency measures. Thus, with regard to production, no large-scale changes are proposed. On the contrary, with resource-oriented scenario specification and SCA, a sophisticated policy mix would be required, to provide incentives and resources to restructure food systems towards systems where resources are allocated such that humans fed per hectare are again in focus. In total, this would require a focus on closed nutrient flows and circular economy concepts. For animal-source food (ASF), this would mean a focus on low-opportunity-cost livestock (LOCL), leading to requirements for breeding programmes, feed processing, etc. These differing implications are of particular importance, because choosing an inadequate methodological approach for the problem of interest (see first point above) could result in inadequate recommendations for policies.

Third, for civil society, our results give insights into congruent and differing dietary solutions, and for the latter offer possible reasons by making underlying - and often implicit - assumptions transparent. Although all reviewed studies agree on a reduction of ASF, differences occur: while studies operating within current settings generally recommend to reduce beef most, followed by pork and chicken, studies considering consequences of dietary change, co-product links, and restrictions on resource use and sink capacities tend to recommend a role for livestock based on the LOCL principle. Thus, for researchers, policy makers, and civil society, implications differ between methodological approaches. Choosing the correct methodological approach is therefore central for avoiding confusion, and for effective communication and policy design. 


\section{Acknowledgements}

The contribution of AF, AM, and CS for this work was supported by the Swiss National Science

Foundation (National Research Project 69: "Sustainable and healthy diets: Trade-offs and synergies", project 4069-166765). 
26 


\title{
Chapter 3
}

\section{How food choices link sociodemographic and lifestyle factors with sustainability impacts}

\author{
A. Frehnera,b, H.H.E. Van Zanten ${ }^{b}$, C. Schadera, I.J.M. De Boerb, G. Pestonid ${ }^{\mathrm{d}}$, S. Rohrmann ${ }^{\mathrm{d}}$, \\ A. Mullera,e
}

aDepartment of Socioeconomics, Research Institute of Organic Agriculture FiBL, Frick, Switzerland

bAnimal Production Systems group, Wageningen University \& Research, Wageningen, the Netherlands

dDivision of Chronic Disease Epidemiology, Epidemiology, Biostatistics and Prevention Institute, University of Zurich, Zurich, Switzerland

eInstitute of Environmental Decisions, Federal Institutes of Technology Zurich ETHZ, Zurich, Switzerland

Journal of Cleaner Production 300 (2021) 126896 


\section{Abstract}

While the production of food causes major environmental impacts and poses social risks, consumption of healthy and nutritious food is essential for human wellbeing. Against this background, action to make current diets more sustainable is needed, which in turn requires knowledge on possibilities for improvement. In this study, we investigated how sociodemographic and lifestyle factors relate to different sustainability impacts of diets in Switzerland using recent dietary recall data $(n=2,057)$. Of each dietary recall, we assessed six impacts: global warming potential, cropland and grassland occupation, social risks, diet quality, and diet cost. We investigated the association between sociodemographic and lifestyle factors and food choices as well as between sociodemographic and lifestyle factors and environmental and socio-economic impacts, and combined these results in a qualitative approach. The median impacts of Swiss dietary recalls were $3.25 \mathrm{~kg} \mathrm{CO}$ eq for global warming potential, $4.92 \mathrm{~m}^{2}$ for cropland occupation, and $1.43 \mathrm{~m}^{2}$ for grassland occupation. Further, the median score for social risks was 1.64 e+08 points (Social Hotspots Index), for diet quality 43.65 points (Alternate Healthy Eating Index), and 9.27 CHF for diet cost. Moreover, our results showed that any action on food groups, be it for health, social, or environmental reasons, potentially affects societal groups differently. Nationalities, language regions, age groups, and smoking status seemed particularly distinctive, while income or educational groups seemed hardly relevant. Further, reductions of some food groups, especially different types of meat, offer large potentials for synergies on multiple impact categories. Others, such as fruits and vegetables as well as fish and seafood, result in trade-offs. On the one hand, these food groups contribute to an improved diet quality. On the other hand, these food groups are costly, and the production of fruits and vegetables additionally poses social risks. Our results contribute to target measures to support environmentally-friendly, healthy, and social diets more effectively. 


\section{Introduction}

While consuming healthy and nutritious food is essential for human wellbeing, the associated food production causes adverse impacts on the environment and poses social risks (Poore and Nemecek, 2018; Willett et al., 2019). Agriculture contributes substantially to approaching or transgressing several planetary boundaries (Campbell et al., 2017; Steffen et al., 2015). In fact, agriculture's share of anthropogenic greenhouse gas (GHG) emissions is currently around 23\%, including agriculturally-driven land use change (Intergovernmental Panel on Climate, 2019). Around 40\% of earth's land surface is used for croplands and pastures (Foley et al., 2011). Food production, however, not only threatens the environment in multiple ways, but also causes social risks, e.g. related to labour conditions (Benoit-Norris et al., 2012). Moreover, diets link impacts caused by food production with food consumption, and thus via diet quality with impacts on human health (Tilman and Clark, 2014). Several studies found that certain dietary patterns increase the risk for noncommunicable diseases, such as diabetes, cardiovascular disease, stroke, and cancer (Afshin et al., 2019; Organization, 2017). Against this background, action to make current diets more sustainable is needed, which in turn requires knowledge on possibilities for improvement.

Much recent literature has focused on dietary changes that reduce environmental pressures, and at the same time improve diet quality (Aleksandrowicz et al., 2016; Willett et al., 2019). Dietary changes are mostly assessed in scenario analysis, which range from predefined scenarios to optimised scenarios. Predefined scenarios, on the one hand, can further be divided into consumption-oriented and resource-oriented scenarios (Frehner et al., 2020). Examples for consumption-oriented predefined scenarios are assessments of dietary guidelines (Springmann et al., 2020); examples for resource-oriented predefined scenarios are scenarios that consider the suitability of resources, such as limiting animal-source foods to the extent that can be produced based on low-opportunity cost biomass (Van Zanten et al., 2018). On the other hand, diets in optimised scenarios for example minimise dietary costs or certain environmental impacts (Benvenuti et al., 2019; Ferrari et al., 2020), or the difference to current diets, while fulfilling certain environmental goals (Gazan et al., 2018). This form of scenarios can indicate short-term improvements of current diets, which are effective yet straightforward to implement. However, most of these studies do not reflect realistic dietary choices (Hallström et al., 2015). In fact, consumers' acceptability is substantially limiting the extent to which for example GHG emissions can be reduced through dietary change (Macdiarmid et al., 2012). Further, as dietary choices differ between population (sub)groups (Hallström and Börjesson, 2013; Hiza et al., 2013), information on these differences can contribute to target nutritional campaigns for improving multiple sustainability impacts (Snyder, 2007). Moreover, information on typical food choices per population (sub)group can provide important information on entry points for actions towards more sustainable diets. As an example, Vassallo et al. (2016) identified psychosocial determinants that influence sustainable food choices. Then, Mozaffarian et al. (2018) mapped the different areas of influence on dietary choices, ranging from sociocultural over governmental until global level. These areas can be targeted most effectively if also the individual layer is taken into account, which consists of multiple sociodemographic and lifestyle factors. Hence, sustainability assessments of current diets can, if combined with information about 
sociodemographic and lifestyle factors, add realism to the discussion on sustainable diets, and thereby provide information for potential pathways for improvement.

While certain consequences of human diets, such as on the environment as well as on human health, have been thoroughly investigated, other dimensions of sustainability are less frequently investigated (Meybeck and Gitz, 2017). This differing level of attention can not only be linked to political agendas (e.g. the strong focus on climate change), but also to the degree of standardisation of impact assessments. The assessment of GHG emissions, for example, is highly standardised thanks to large efforts of the Intergovernmental Panel on Climate Change community, thereby facilitating straightforward comparison between assessments. Other impact categories within the environmental dimension are still assessed in many different ways, such as impacts on biodiversity. Similarly, impacts within the social dimension of sustainability are not yet as standardised as GHG emissions, and are not that commonly assessed. Nevertheless, the social dimension of agricultural production constitutes a highly challenging and relevant area. Currently, over 3 billion people live in rural areas (FAO, 2018), of which around 2.5 billion people derive their livelihood from the sector agriculture (FAO, 2013). Including the social dimension in sustainability assessments is therefore key for a profound multidisciplinary understanding of the impacts of our diets. According to Huarachi et al. (2020), social impact assessment is currently in the phase of the search for standardisation, and is still far from scientific maturity. The currently most commonly used database for such assessments is the Social Hotspots Database (SHDB) (Huarachi et al., 2020).

Hence, we lack an integrated sustainability assessment of different actual diets, that can be linked to information on population (sub)groups. To this end, we investigated in this study how sociodemographic and lifestyle factors relate to different environmental and socio-economic impacts of diets. The relation between sociodemographic and lifestyle factors and impacts is clearly not a direct one, as it is mediated via the food choices and the related impacts. It nevertheless adds an important level to the analysis, since much of the debate on sustainable food consumption relates to differences between socio-demographic groups (Mohr and Schlich, 2016). We conducted an integrated assessment of individual dietary recall data from Switzerland, including multiple aspects of environmental and socio-economic sustainability. On the production side, we assessed the environmental indicators global warming potential (GWP), cropland occupation (CLO), and grassland occupation (GLO) ${ }^{1}$, as well as social risks by employing the Social Hotspots Index (SHI), based on the SHDB (Benoit-Norris et al., 2012). On the consumption side, diet quality was assessed by the Alternate Healthy Eating Index (AHEI) (Chiuve et al., 2012; Pestoni et al., 2019), and further, costs of the diets were assessed. Then, we investigated the associations between population characteristics and food choices on the one hand, and between population characteristics and sustainability impacts on the other hand by means of multiple linear regression. Subsequently, we combined these results in a qualitative manner. Thereby, we gain a detailed picture on which sociodemographic groups may significantly differ in impacts and which of their food choices are determinant for this.

\footnotetext{
${ }^{1}$ Global warming potential: also referred to as greenhouse gas emissions in this thesis.

Land occupation: also referred to as land use in this thesis.
} 
In order to study effects within a defined region, we employed Switzerland as a case study. Since dietary recalls are mostly carried out at national level with differing methodologies, comparisons between countries are challenging. With its three language regions, which are influenced by the respective neighbouring countries, Switzerland offers a unique opportunity to study different cultural settings within one harmonised dietary recall dataset (Pestoni et al., 2019).

\section{Material and methods}

\section{I Dietary recall data and sociodemographic and lifestyle variables}

The first, and also most recent, dietary recall in Switzerland (menuCH) was conducted between January 2014 and February 2015 (Chatelan et al., 2017a). A total of 2,057 participants completed two $24 \mathrm{~h}$ dietary recalls, reporting all food items consumed within the last 24 hours. Food items were entered as detailed as possible (e.g. tomatoes) and subsequently grouped to food categories (e.g. vegetables). In addition to this, sociodemographic information and lifestyle variables were collected, of which the following will be used in this analysis: sex, nationality, age group, highest completed education, civil status, gross household income, smoking status, and being currently on a weightloss diet. Further, weight and height were measured to derive the Body Mass Index (BMI). The language region was determined depending on the canton of residence.

In combination with a weighting scheme accounting for sex, age, nationality, civil status, household size, major area in Switzerland, weekdays, and seasonality, this sample became representative for inhabitants between the age of 18 and 75 and for their consumption habits over the year for most regions in Switzerland (Pasquier et al., 2017).

The consumed amounts of each food product in the dietary recalls had to be transformed in order to be consistent with the reference units of the environmental impact intensities employed (Appendix B1). In short, we disentangled recipes to ingredients level where required. Then, we converted cooked amounts to raw amounts, thus mainly adjusting the water content of food products. Further, we added factors to account for food waste until the stage of consumption (Beretta et al., 2017), with which we obtained food demand values from food intake values. Finally, food groups used in menuCH were mapped and aggregated to commodity groups used in the impact assessment.

\subsection{Environmental and socio-economic impact assessment}

For each dietary recall, we assessed multiple environmental and socio-economic impacts. To represent impacts on the environment, we selected the three impact categories global warming potential (GWP), cropland occupation (CLO), and grassland occupation (GLO). Further, for socioeconomic impact categories, we assessed social risks on the production side using the Social Hotspots Index (SHI), and on the consumption side, cost of food consumption as well as the diet quality score Alternate Healthy Eating Index (AHEI). 


\section{2 | Chapter 3}

\subsubsection{Environmental impact assessment}

We calculated life cycle assessments from cradle-to-farm-gate with the biophysical mass-flow model SOLm (Muller et al., 2017; Schader et al., 2015), and from farm-gate-to-consumer (covering the stages processing and transport), we employed inventories from Ecoinvent 3 (Appendix B2). SOLm depicts all mass and nutrient flows in agricultural production that are relevant for the calculation of resource use and emissions. In total, SOLm covers 192 countries, 180 primary crop and 22 primary livestock activities as defined in FAOSTAT, which are further mainly calibrated with FAOSTAT data, such as food balance sheets (FAOSTAT, 2018). For this study, we employed data for Switzerland and its importing countries.

For each crop and livestock activity, inputs and outputs are defined and comprise all processes included in the inventories. For crop activities, the following inputs are included: land area (distinguished whether occupied for crops or grass), mineral and organic fertiliser (manure, crop residues), nitrogen fixation, pesticides, management practices, and processing. Outputs comprise crop yields (main and by-products), and residues. GHG emissions of crop production included emissions during production, processing, and transport. For livestock activities, the inputs feed and energy use for housing and fences were included. Covered outputs are products (human-edible; meat, milk, eggs, and human-inedible; skins, hides, bones, etc.), as well as manure excretion. Thus, GHG emissions of feed production, enteric fermentation, manure management, processing, and transport were covered. Based on these inventories, we assessed GWP, CLO, and GLO per food item.

\subsubsection{Social risks}

We assessed social risks based on the Social Hotspots Database (SHDB) (Benoit-Norris et al., 2012). The SHDB consists of 156 social indicators per country and per sector of the following areas: labour rights and decent work, health and safety, human rights, governance, and community infrastructure. The agricultural sector is divided into 22 sub-sectors, whereof 18 are directly related to food production. The social indicators were aggregated to the SHI according to a proposed weighting scheme (Benoit-Norris et al., 2012; Norris et al., 2014) (Appendix B2). Indirect social impacts were estimated using an input-output table for Switzerland (Nathani et al., 2011) that contains the interlinkages between multiple industries and between industries and final demand of an economy. By this, also social impacts of sectors that provide inputs into agricultural sectors, such as pesticide production, could be captured. A higher score in SHI indicates a higher social risk in production and along the value chain.

\subsubsection{Cost of food consumption}

We collected price data of 94 commodities at one day (Appendix B2). Then, by multiplying food demand values with prices, we estimated total cost of food demand per dietary recall. 


\subsubsection{Diet quality}

Impacts on human health of food consumption differ from individual to individual, depending on a combination of factors, such as the prevailing gut microbiota (Clemente et al., 2012). Nevertheless, indices can give helpful insights into correlations of certain food groups with improved or decreased health performance. We employed the AHEI (Chiuve et al., 2012; Pestoni et al., 2019), a diet quality score which correlates well with coronary heart disease, diabetes, and risk of stroke and cancer (Schwingshackl et al., 2018; Waijers et al., 2007). The AHEI is derived from the consumed amounts of 11 food categories, where various intake thresholds define the amount of points that are allocated to the observed individual consumption levels, leading to a maximum achievable score of 110 points. A higher AHEI indicates a higher diet quality.

To sum up, we calculated environmental and socio-economic impacts by consumed food group by person as well as for the whole diet per person. We assessed diet quality for each person for the whole diet only (not per food group), since this impact category depends on the composition of a person's diet. Then, we dichotomised impact intensities (above and below median; strong and weak) for each impact category and food group, to classify the strength per food group and impact category. Since the AHEI is calculated at diet level, additional steps were required to define strong and weak contribution of single food groups to the AHEI (Appendix B2).

\subsection{Statistical analysis and integration}

\subsection{Sociodemographic and lifestyle factors and food choices}

In a first step, we used multiple linear regression to identify typical food choices for different sociodemographic and lifestyle factors, with both the food choices (g consumed per food group) and the sociodemographic and lifestyle factors coming from the menuCH dataset (Section 2.1). Individual regressions were fitted per food group, with consumption per food group as dependent variables, and the sociodemographic variables and lifestyle factors as explanatory variables (Appendix B3). In addition, food energy intake was included as adjusting factor in each model, to correct for different levels of absolute food energy intake in the dietary recalls. The dietary recall data was weighted according to the weighting scheme described in Section 2.1. Only results of the regression analysis that met our criteria for significance ( $p$-value $\leq 0.05$ ) were used for the subsequent analysis.

\subsubsection{Sociodemographic and lifestyle factors and impacts}

In a second step, as for the food groups, we used multiple linear regression to investigate associations between sociodemographic and lifestyle factors and environmental and socio-economic impacts. While the sociodemographic and lifestyle factors were taken from the menuCH dataset, the environmental and socio-economic impacts were based on own calculations with indicated impact assessment methods (Section 2.2). For each impact category, an individual regression was fitted, including food energy intake to correct for different levels of food energy intakes of the diets. Dietary recalls were weighted according to the described weighting scheme in Section 2.1. Further, SHI was 


\section{4 | Chapter 3}

log transformed, which improved model-fitting performance. Also here, results were processed further for defined levels of significance only ( $p$-value $\leq 0.05$ ).

\subsubsection{Integrating sociodemographic and lifestyle factors, food choices, and impacts}

In a third step, we combined results of the regression analyses for food choices and impacts (Sections 2.3.1 and 2.3.2) to obtain a full picture of how food choices link sociodemographic and lifestyle factors with impact levels. To start with, we extracted sociodemographic and lifestyle factors that showed statistically significant results ( $p$-value $\leq 0.05$ ) for each impact category. Then, we took the direction of the association (positive and negative) between the extracted sociodemographic and lifestyle factors and the food groups, and combined it with the information on the dichotomised impact intensities, i.e. the strength per food group and impact category (strong and weak). By this, we identified relevant food choices among sociodemographic and lifestyle groups and their influence on the different impact categories.

\section{Results and discussion}

\section{I Environmental and socio-economic impacts of food groups}

Figure 1 presents the mean impacts for AHEI, cost, GWP, GLO, CLO, and SHI of the weighted dietary recalls from the menuCH dataset. The scores refer to the sum of all foods consumed by an individual in the 24 hours of the recalls. The median of the AHEI was 43.65 points, the median of cost amounted to $9.27 \mathrm{CHF}$, while the medians of the environmental indicators were $3.25 \mathrm{~kg} \mathrm{CO} e \mathrm{eq}$ for GWP, $1.43 \mathrm{~m}^{2}$ for GLO, and $4.92 \mathrm{~m}^{2}$ for CLO. Further, the median score for the SHI was $1.64 \mathrm{e}+08$ points.

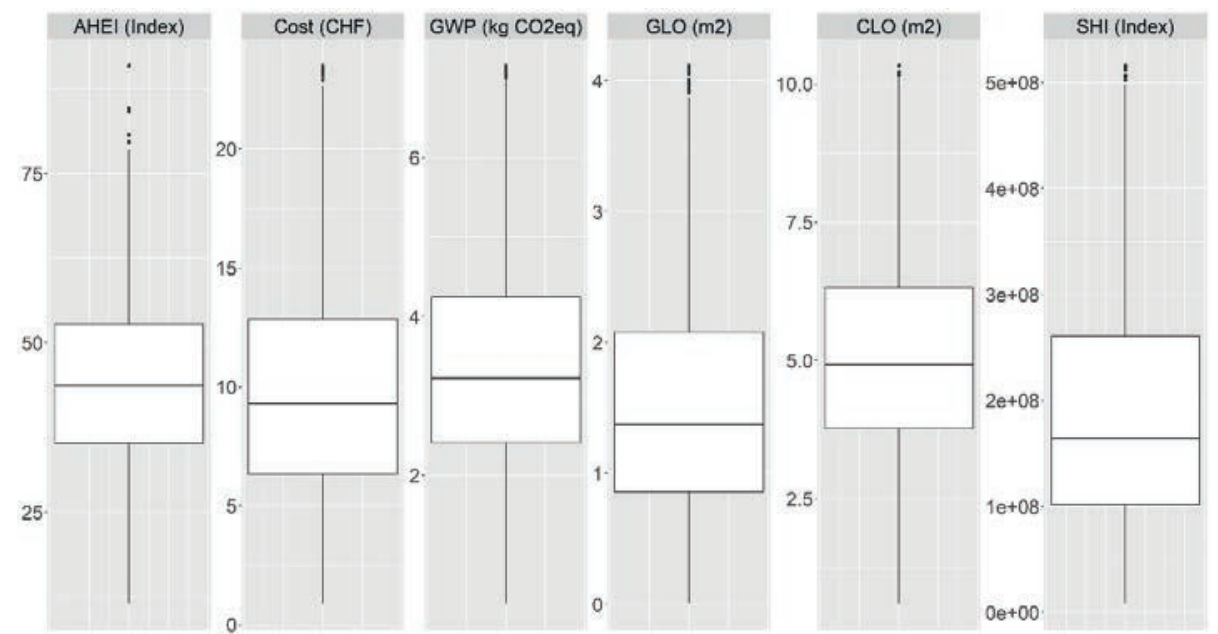

Figure I. Weighted boxplots of the impacts of the foods consumed by an individual in the 24 hours of the recall. AHEI $=$ Alternate Healthy Eating Index, GWP = global warming potential, GLO = grassland occupation, CLO = cropland occupation, SHI = Social Hotspots Index. 
Results of the dichotomised impact intensities per food group are presented in Figure 2. We see that for the AHEI, mainly the food groups fish and seafood, cereals (whole grains), pulses, vegetables, nuts and seeds, and fruits contributed to an increase of the score (higher score refers to higher diet quality). For cost, on the contrary, animal-source food (ASF), and especially the different types of meat, contributed strongly to an increase, as well as fish and seafood, vegetables, fruits, and alcoholic beverages. For GWP, mainly the ASF categories revealed above median impact intensities, accompanied by oils and fats. Impact intensities of GWP and CLO were fairly well aligned, with the exception of milk products, pulses, and nuts and seeds. Only ruminants were fed with grass resources and thus occupied land for grass, and therefore, GLO was only relevant for cattle meat and milk products. Finally, results for the SHI revealed a different picture, with, next to eggs and fish and seafood, mainly plant-source food contributing to an increased score (a higher score refers to higher social risks along the value chain).

For the subsequent analysis, we employed results of the sustainability impact assessment on diets level and information on the dichotomised impact intensities. Although the different nutritional functions of each food item - be it dairy as a source for calcium, or carrots as a source of vitamin A - were thus not captured, this simplification is less relevant when the strength of the impact intensity per food group is considered in a dichotomised way only. Supporting this decision, Van Kernebeek et al. (2014) found only small differences when relating GWP to a nutritional quality score instead of day or kg of product, and no difference for LO.

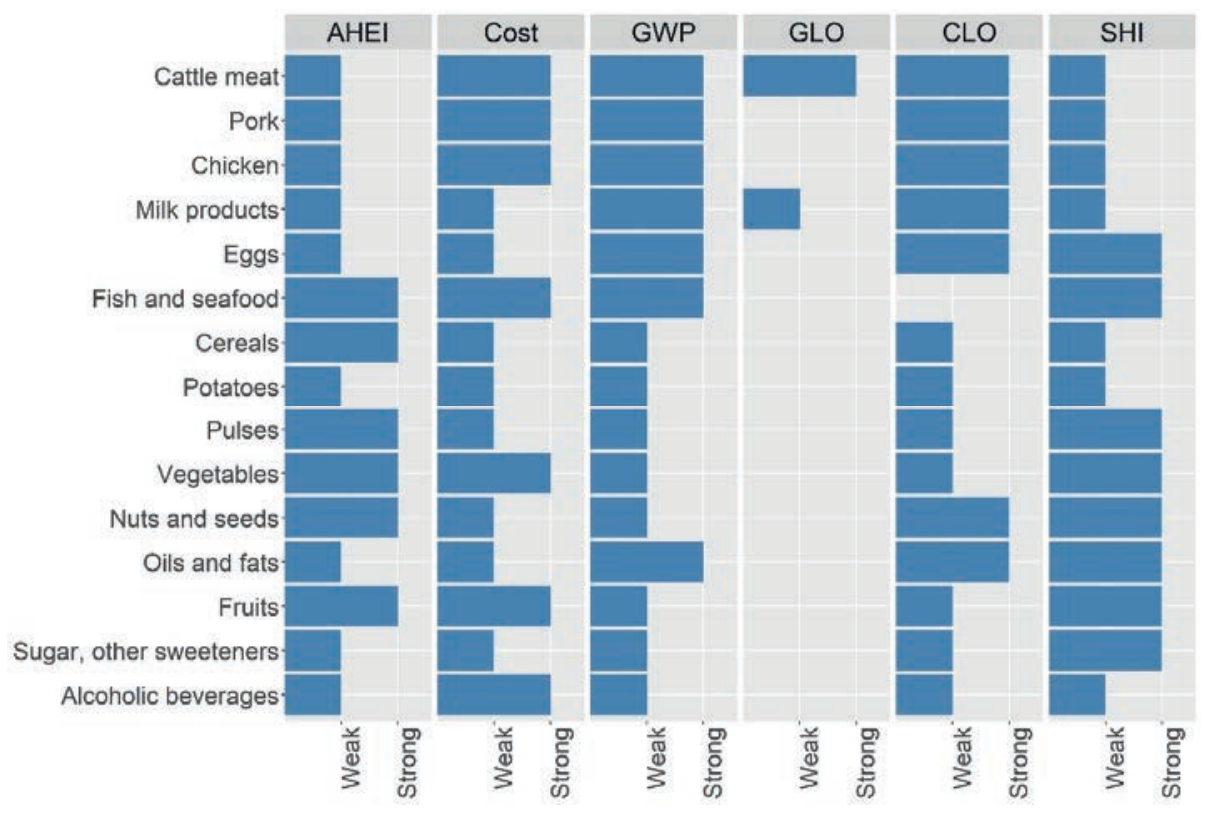

Figure 2. Dichotomised impact intensities per food group (above median: strong, below median: weak). AHEI = Alternate Healthy Eating Index, GWP = global warming potential, GLO = grassland occupation, CLO = cropland occupation, SHI = Social Hotspots Index. 


\section{6 | Chapter 3}

\subsection{Results on statistical analysis and integration}

\subsection{Sociodemographic and lifestyle factors and food choices}

Regression analysis revealed several relevant sociodemographic and lifestyle drivers of food choices (Tables 1 and 2). For the consumption of different types of meat, all drivers except income group and being currently on a diet showed significant results for at least one meat type. Further, consumption of milk products, eggs, and fish and seafood showed significant results for the drivers sex, nationality, language region, age group, civil status, as well as smoking.

Table I. Association between sociodemographic and lifestyle factors and animal-source food groups. Coefficient estimate; [95\% Cl]; p-value; bold: results with a p-value below or equal to $0.05(n=2,057)$.

\begin{tabular}{|c|c|c|c|c|c|c|c|c|c|}
\hline \multirow{2}{*}{$\begin{array}{l}\text { Covariate (Reference) } \\
\text { Sex (Female) }\end{array}$} & \multicolumn{2}{|c|}{ Cattle meat } & \multicolumn{3}{|c|}{ Pork } & \multicolumn{3}{|c|}{ Chicken } & \multirow[b]{3}{*}{0.00} \\
\hline & & & & & & & & & \\
\hline Male & 16.52 & {$[4.17 ; 28.88]$} & 0.01 & -3.03 & {$[-18.04,11.98]$} & 0.69 & 22.95 & {$[7.94,37.95]$} & \\
\hline \multicolumn{10}{|l|}{ Nationality (Swiss) } \\
\hline European & 2.89 & {$[-10.11,15.88]$} & 0.66 & 6.64 & {$[-9.33,22.61]$} & 0.41 & -0.76 & {$[-16.23,14.72]$} & 0.92 \\
\hline Region of the & -30.56 & {$[-91.17,30.05]$} & 0.32 & 115.46 & {$[2.43,228.48]$} & 0.05 & 9.37 & {$[-35.89,54.63]$} & 0.68 \\
\hline \multicolumn{10}{|l|}{ Americas } \\
\hline African / Eastem Meditemanean & 52.83 & {$[6.65,99]$} & 0.03 & -13.54 & {$[-94.06,66.98]$} & 0.74 & 5.69 & {$[-53.14,64.52]$} & 0.85 \\
\hline Westem Pacific / & 47.05 & {$[18.48,75.63]$} & 0.00 & & & & 27.94 & {$[-1.62,57.51]$} & 0.06 \\
\hline \multicolumn{10}{|l|}{ South-East Asia } \\
\hline \multicolumn{10}{|l|}{$\begin{array}{l}\text { Language region } \\
\text { (German-speaking) }\end{array}$} \\
\hline French-speaking & 17.03 & {$[5.41,28.64]$} & 0.00 & -12.91 & {$[-28.15,2.33]$} & 0.10 & 22.85 & {$[8.44,37.25]$} & 0.00 \\
\hline Ita lian-spea king & 13.10 & {$[-8.6,34.8]$} & 0.24 & 1.12 & {$[-34.11,36.35]$} & 0.95 & 14.96 & {$[-13.98,43.89]$} & 0.31 \\
\hline \multicolumn{10}{|l|}{ Age group ( $30-44$ years) } \\
\hline $18-29$ years & 9.86 & {$[-11.23,30.95]$} & 0.36 & -13.16 & {$[-40.26,13.95]$} & 0.34 & 26.04 & {$[3.56,48.52]$} & 0.02 \\
\hline $45-59$ years & -5.74 & {$[-19.22,7.75]$} & 0.40 & -7.79 & {$[-24,8.42]$} & 0.34 & -19.31 & {$[-36.13,-2.48]$} & 0.03 \\
\hline $60-75$ years & 0.73 & {$[-16.5,17.95]$} & 0.93 & 0.37 & {$[-19.98,20.72]$} & 0.97 & -23.95 & {$[-45.89,-2]$} & 0.03 \\
\hline \multicolumn{10}{|l|}{$\begin{array}{l}\text { Education; highest } \\
\text { degree (Secondary) }\end{array}$} \\
\hline Primary & 5.34 & {$[-33.83,44.51]$} & 0.79 & 2.94 & {$[-30.26,36.14]$} & 0.86 & -4.43 & {$[-40.48,31.62]$} & 0.81 \\
\hline Tertiary & -13.20 & {$[-23.89,-2.52]$} & 0.02 & -2.58 & {$[-15.96,10.79]$} & 0.70 & -9.46 & {$[-22.97,4.05]$} & 0.17 \\
\hline \multicolumn{10}{|l|}{ Civil status (Single) } \\
\hline Mamied & 21.01 & {$[2.97,39.04]$} & 0.02 & 2.46 & {$[-19.14,24.06]$} & 0.82 & -0.55 & {$[-21.25,20.14]$} & 0.96 \\
\hline Divorced & -3.07 & {$[-27.23,21.09]$} & 0.80 & 0.20 & {$[-27.15,27.56]$} & 0.99 & 23.84 & {$[-0.85,48.53]$} & 0.06 \\
\hline Other & -0.19 & {$[-57.1,56.72]$} & 1.00 & 9.39 & {$[-33.2,51.98]$} & 0.66 & 8.27 & {$[-23.56,40.1]$} & 0.61 \\
\hline \multicolumn{10}{|l|}{ Inc ome group $(6,000$} \\
\hline \multicolumn{10}{|l|}{ - $13,000 \mathrm{CHF} /$ month) } \\
\hline$<6,000 \mathrm{CHF} /$ month & 7.21 & [-8.02,22.43] & 0.35 & 10.28 & [-7.62,28.18] & 0.26 & -16.45 & {$[-34.11,1.21]$} & 0.07 \\
\hline$>13,000 \mathrm{CHF} /$ month & 6.18 & {$[-6.87,19.23]$} & 0.35 & 0.84 & {$[-17.3,18.97]$} & 0.93 & 12.76 & {$[-3.26,28.78]$} & 0.12 \\
\hline \multicolumn{10}{|l|}{ Smoking status (No) } \\
\hline Current & 15.85 & {$[2.45,29.25]$} & 0.02 & 5.76 & [-11.4,22.92] & 0.51 & -17.03 & {$[-33.55,-0.51]$} & 0.04 \\
\hline Former & 0.50 & {$[-12.01,13]$} & 0.94 & -14.16 & {$[-29.28,0.96]$} & 0.07 & -2.44 & {$[-16.96,12.08]$} & 0.74 \\
\hline \multicolumn{10}{|l|}{ BMI group (Nomal) } \\
\hline Underweight & 19.57 & {$[-38.54,77.67]$} & 0.51 & -54.87 & {$[-109.24,-0.5]$} & 0.05 & -7.26 & {$[-57.67,43.15]$} & 0.78 \\
\hline Overweight & 5.13 & {$[-6.49,16.75]$} & 0.39 & -2.16 & {$[-16.47,12.16]$} & 0.77 & 2.85 & {$[-11.21,16.91]$} & 0.69 \\
\hline Obese & -2.63 & {$[-19.06,13.81]$} & 0.75 & -6.64 & {$[-26.03,12.75]$} & 0.50 & 4.58 & {$[-17.49,26.66]$} & 0.68 \\
\hline \multicolumn{10}{|l|}{ Cumently on a weight-loss diet (No) } \\
\hline Yes & 8.04 & {$[-13.72,29.8]$} & 0.47 & 2.62 & {$[-32.29,37.54]$} & 0.88 & 16.11 & {$[-9.15,41.36]$} & 0.21 \\
\hline
\end{tabular}


Table I (continued). Association between sociodemographic and lifestyle factors and animal-source food groups. Coefficient estimate; [95\% Cl]; p-value; bold: results with a p-value below or equal to $0.05(n=2,057)$.

\begin{tabular}{|c|c|c|c|c|c|c|c|c|c|}
\hline \multirow{2}{*}{$\begin{array}{l}\text { Covariate (Reference) } \\
\text { Sex (Female) }\end{array}$} & \multicolumn{2}{|c|}{ Milk products } & \multicolumn{3}{|c|}{ Eggs } & \multicolumn{4}{|c|}{ Fish and seafood } \\
\hline & & & & & & & & & \\
\hline Male & -29.68 & {$[-49.2,-10.16]$} & 0.00 & 1.39 & {$[-2.69,5.48]$} & 0.50 & 4.60 & {$[-7.13,16.34]$} & 0.44 \\
\hline \multicolumn{10}{|l|}{ Nationality (Swiss) } \\
\hline European & -47.21 & {$[-68.55,-25.87]$} & 0.00 & 7.38 & {$[2.9,11.86]$} & 0.00 & 19.03 & {$[7.3,30.76]$} & 0.00 \\
\hline Region of the & -20.26 & {$[-122.33,81.82]$} & 0.70 & -16.15 & {$[-39.93,7.62]$} & 0.18 & -9.19 & {$[-65.74,47.37]$} & 0.75 \\
\hline \multicolumn{10}{|l|}{ Americas } \\
\hline African / Eastem Mediterranean & -70.43 & {$[-162.96,22.09]$} & 0.14 & -8.58 & {$[-28.01,10.85]$} & 0.39 & -23.45 & {$[-85.98,39.07]$} & 0.46 \\
\hline Westem Pacific / & 22.16 & {$[-38.46,82.78]$} & 0.47 & -9.74 & {$[-22.04,2.57]$} & 0.12 & -21.38 & {$[-45.22,2.46]$} & 0.08 \\
\hline \multicolumn{10}{|l|}{ South-East Asia } \\
\hline \multicolumn{10}{|l|}{ Language region } \\
\hline French-speaking & -65.92 & {$[-85.98,-45.86]$} & 0.00 & 3.23 & {$[-0.99,7.45]$} & 0.13 & 4.99 & {$[-5.89,15.87]$} & 0.37 \\
\hline Italian-spea king & -56.92 & {$[-97.3,-16.55]$} & 0.01 & -4.88 & {$[-13.31,3.54]$} & 0.26 & 7.97 & {$[-12.46,28.41]$} & 0.44 \\
\hline \multicolumn{10}{|l|}{ Age group (30-44 years) } \\
\hline $18-29$ years & -1.51 & {$[-32.3,29.27]$} & 0.92 & 7.27 & {$[0.78,13.76]$} & 0.03 & 4.52 & {$[-14.71,23.75]$} & 0.65 \\
\hline $45-59$ years & -11.10 & {$[-34.22,12.01]$} & 0.35 & 3.10 & {$[-1.74,7.93]$} & 0.21 & 2.02 & {$[-10.93,14.97]$} & 0.76 \\
\hline $60-75$ years & 0.57 & {$[-28.07,29.2]$} & 0.97 & 2.97 & {$[-3,8.95]$} & 0.33 & 13.81 & {$[-3.1,30.72]$} & 0.11 \\
\hline \multicolumn{10}{|l|}{$\begin{array}{l}\text { Education; highest } \\
\text { degree (Sec ondary) }\end{array}$} \\
\hline Primary & 1.19 & {$[-50.06,52.44]$} & 0.96 & 2.58 & {$[-8.31,13.47]$} & 0.64 & 7.10 & {$[-21.3,35.49]$} & 0.62 \\
\hline Tertiary & 9.14 & {$[-9.21,27.49]$} & 0.33 & -1.75 & {$[-5.62,2.13]$} & 0.38 & -4.86 & {$[-15.62,5.89]$} & 0.38 \\
\hline \multicolumn{10}{|l|}{ Civil status (Single) } \\
\hline Mamied & 30.74 & {$[1.63,59.86]$} & 0.04 & 2.96 & {$[-3.15,9.06]$} & 0.34 & 2.72 & {$[-14.84,20.27]$} & 0.76 \\
\hline Divorced & 16.23 & {$[-18.27,50.74]$} & 0.36 & -5.32 & {$[-12.62,1.97]$} & 0.15 & 1.08 & {$[-19.07,21.24]$} & 0.92 \\
\hline Other & -31.98 & {$[-78.34,14.38]$} & 0.18 & 0.22 & {$[-9.7,10.15]$} & 0.97 & -7.36 & {$[-32.13,17.42]$} & 0.56 \\
\hline \multicolumn{10}{|l|}{ Income group $(6,000$} \\
\hline \multicolumn{10}{|l|}{ - $13,000 \mathrm{CHF} /$ month) } \\
\hline$<6,000 \mathrm{CHF} /$ month & 16.72 & {$[-5.76,39.21]$} & 0.15 & 0.25 & {$[-4.48,4.98]$} & 0.92 & -11.50 & {$[-25.48,2.48]$} & 0.11 \\
\hline$>13,000 \mathrm{CHF} /$ month & 7.80 & {$[-14.67,30.27]$} & 0.50 & -1.24 & {$[-5.98,3.5]$} & 0.61 & 1.75 & {$[-10.67,14.17]$} & 0.78 \\
\hline \multicolumn{10}{|l|}{ Smoking status (No) } \\
\hline Current & -19.18 & {$[-41.86,3.49]$} & 0.10 & -1.29 & {$[-6.05,3.48]$} & 0.60 & -4.76 & {$[-18.14,8.63]$} & 0.49 \\
\hline Former & -24.88 & {$[-44.2,-5.57]$} & 0.01 & 1.89 & {$[-2.15,5.94]$} & 0.36 & -7.19 & {$[-18.55,4.18]$} & 0.21 \\
\hline \multicolumn{10}{|l|}{ BMI group (Nomal) } \\
\hline Underweight & -11.42 & {$[-73.52,50.68]$} & 0.72 & -2.99 & {$[-16.51,10.53]$} & 0.66 & 9.13 & {$[-28.75,47.02]$} & 0.64 \\
\hline Overweight & 3.88 & {$[-15.89,23.66]$} & 0.70 & 2.99 & {$[-1.17,7.14]$} & 0.16 & -2.80 & {$[-14.23,8.62]$} & 0.63 \\
\hline Obese & -11.59 & {$[-39.18,16.01]$} & 0.41 & -1.42 & {$[-7.23,4.4]$} & 0.63 & 5.17 & {$[-12 \cdot 1,22.44]$} & 0.56 \\
\hline \multicolumn{10}{|l|}{ Cumently on a weight-loss diet (No) } \\
\hline Yes & 22.12 & {$[-18.66,62.9]$} & 0.29 & 3.08 & {$[-5.9,12.07]$} & 0.50 & -8.15 & {$[-27.5,11.2]$} & 0.41 \\
\hline
\end{tabular}

It was interesting to note that while cattle meat and chicken consumption was higher among male participants (+16.5 g and $+23.0 \mathrm{~g})$, milk consumption was higher among female participants. Notably, participants originating from non-European countries had a higher meat intake (cattle meat or pork), while participants from the European region had higher egg intake (+7.4 g) and lower milk intake (-47.2 g), compared to the Swiss reference group. Interestingly, a similar pattern could be observed for Swiss language regions other than the German-speaking reference group: the Frenchspeaking region showed higher consumption of cattle meat $(+17.0 \mathrm{~g})$ and chicken $(+22.9 \mathrm{~g})$, and lower consumption of milk (-65.9 g) - the latter result also holds for participants from the Italian-speaking region (-56.9 g). Between age groups, only consumption of chicken and eggs varied; with both being consumed more by participants younger than the reference age group, and chicken being consumed less by older participants. Finally, while cattle meat consumption was lower among participants with tertiary education (-13.2 g), being married or a current smoker revealed an opposite association (+21.0 g and $+15.9 \mathrm{~g})$.

For the food groups cereals and potatoes, the drivers sex, nationality, civil status, income group, BMI group, and being currently on a diet revealed significant results (Table 2). Moreover, the 


\section{8 | Chapter 3}

consumption of pulses, vegetables, and nuts and seeds revealed significant results for all drivers except smoking status. For the two food groups fruits and sugar, all drivers except civil status, income group, and being currently on a diet showed significant results. Finally, the consumption of alcoholic beverages was significantly associated with the explanatory variables sex, nationality, age group, and smoking status.

Table 2. Association between sociodemographic and lifestyle factors and plant-source food groups. Coefficient estimate; [95\% $\mathrm{Cl}]$; $\mathrm{p}$-value; bold: results with a $\mathrm{p}$-value below or equal to $0.05(\mathrm{n}=2,057)$.

\begin{tabular}{|c|c|c|c|c|c|c|c|c|c|}
\hline \multirow{2}{*}{$\begin{array}{l}\text { Covariate (Reference) } \\
\text { Sex (Female) }\end{array}$} & \multicolumn{3}{|c|}{ Cereals } & \multicolumn{3}{|c|}{ Potatoes } & \multicolumn{3}{|l|}{ Pulses } \\
\hline & & & & & & & & & \\
\hline Male & 15.36 & {$[5.47,25.26]$} & 0.00 & 23.93 & {$[11.58,36.28]$} & 0.00 & 8.66 & {$[1.65,15.67]$} & 0.02 \\
\hline \multicolumn{10}{|l|}{ Nationality (Swiss) } \\
\hline European & -7.75 & {$[-18.54,3.04]$} & 0.16 & 8.57 & {$[-5.33,22.47]$} & 0.23 & -2.75 & $\begin{array}{l}{[-10.03,4.53]} \\
\end{array}$ & 0.46 \\
\hline $\begin{array}{l}\text { Region of the } \\
\text { Americas }\end{array}$ & -1.49 & {$[-53.29,50.31]$} & 0.96 & 48.71 & {$[-9.69,107.11]$} & 0.10 & -30.03 & {$[-77.53,17.47]$} & 0.21 \\
\hline African / Eastem Mediterranean & -5.50 & {$[-52.47,41.46]$} & 0.82 & 79.16 & {$[9.16,149.17]$} & 0.03 & 17.62 & {$[-13.38,48.62]$} & 0.26 \\
\hline $\begin{array}{l}\text { Westem Pacific / } \\
\text { South-East Asia }\end{array}$ & -17.20 & {$[-47.97,13.57]$} & 0.27 & 19.54 & {$[-20.41,59.5]$} & 0.34 & -6.69 & {$[-19.55,6.17]$} & 0.31 \\
\hline \multicolumn{10}{|l|}{$\begin{array}{l}\text { Language region } \\
\text { (Geman-speaking) }\end{array}$} \\
\hline French-speaking & 7.97 & {$[-2.21,18.14]$} & 0.13 & -5.31 & {$[-18.19,7.56]$} & 0.42 & 9.81 & {$[2.83,16.8]$} & 0.01 \\
\hline Ita lian-spea king & 10.03 & {$[-10.26,30.33]$} & 0.33 & -9.95 & {$[-34.24,14.33]$} & 0.42 & 15.42 & {$[-1.36,32.2]$} & 0.07 \\
\hline \multicolumn{10}{|l|}{ Age group (30-44 years) } \\
\hline $18-29$ years & 13.16 & {$[-2.42,28.74]$} & 0.10 & 13.74 & {$[-6.11,33.6]$} & 0.18 & -3.15 & {$[-13.49,7.2]$} & 0.55 \\
\hline $45-59$ years & -9.62 & {$[-21.29,2.06]$} & 0.11 & 3.89 & {$[-10.64,18.42]$} & 0.60 & -1.80 & {$[-9.77,6.17]$} & 0.66 \\
\hline $60-75$ years & -13.01 & {$[-27.51,1.5]$} & 0.08 & 6.58 & {$[-11.09,24.25]$} & 0.47 & 0.96 & {$[-8.55,10.48]$} & 0.84 \\
\hline \multicolumn{10}{|l|}{$\begin{array}{l}\text { Education; highest } \\
\text { degree (Secondary) }\end{array}$} \\
\hline Primary & -6.93 & {$[-32.93,19.08]$} & 0.60 & 26.08 & {$[-9.81,61.97]$} & 0.15 & -11.54 & {$[-28.87,5.79]$} & 0.19 \\
\hline Tertiary & 4.23 & {$[-5.06,13.53]$} & 0.37 & -3.27 & {$[-14.73,8.2]$} & 0.58 & -5.92 & {$[-12.28,0.44]$} & 0.07 \\
\hline \multicolumn{10}{|l|}{ Civil status (Single) } \\
\hline Mamied & 2.09 & {$[-12.6,16.78]$} & 0.78 & -5.04 & {$[-22.72,12.63]$} & 0.58 & -5.37 & {$[-14.55,3.81]$} & 0.25 \\
\hline Divorced & -6.23 & {$[-23.71,11.26]$} & 0.49 & -26.97 & {$[-48.41,-5.54]$} & 0.01 & 5.79 & {$[-5.02,16.6]$} & 0.29 \\
\hline Other & 2.94 & {$[-20.25,26.13]$} & 0.80 & 22.76 & {$[-6.67,52.18]$} & 0.13 & 3.48 & {$[-15.29,22.25]$} & 0.72 \\
\hline \multicolumn{10}{|l|}{$\begin{array}{l}\text { Income group }(6,000 \\
-13,000 \mathrm{CHF} / \text { month) }\end{array}$} \\
\hline$<6,000 \mathrm{CHF} /$ month & 3.69 & {$[-7.71,15.1]$} & 0.53 & 14.86 & {$[0.31,29.4]$} & 0.05 & 2.18 & {$[-5.64,10]$} & 0.58 \\
\hline$>13,000 \mathrm{CHF} /$ month & -6.45 & {$[-17.8,4.89]$} & 0.27 & -5.94 & {$[-20.18,8.29]$} & 0.41 & 0.87 & {$[-6.79,8.54]$} & 0.82 \\
\hline \multicolumn{10}{|l|}{ Smoking status (No) } \\
\hline Current & -2.83 & {$[-14.29,8.63]$} & 0.63 & 4.03 & {$[-10.33,18.38]$} & 0.58 & 1.88 & {$[-6.02,9.78]$} & 0.64 \\
\hline Former & -4.72 & {$[-14.5,5.06]$} & 0.34 & -1.50 & {$[-13.68,10.69]$} & 0.81 & -0.46 & {$[-7.15,6.23]$} & 0.89 \\
\hline \multicolumn{10}{|l|}{ BMI group (Nomal) } \\
\hline Underweight & 15.95 & {$[-15.57,47.47]$} & 0.32 & 8.39 & {$[-38.72,55.5]$} & 0.73 & 4.49 & {$[-14.14,23.11]$} & 0.64 \\
\hline Overweight & -11.35 & {$[-21.37,-1.34]$} & 0.03 & 1.53 & {$[-10.89,13.94]$} & 0.81 & 1.81 & {$[-5.22,8.85]$} & 0.61 \\
\hline Obese & -13.48 & {$[-27.5,0.55]$} & 0.06 & 16.10 & {$[-1.65,33.86]$} & 0.08 & 0.55 & {$[-9.47,10.58]$} & 0.91 \\
\hline \multicolumn{10}{|l|}{ Cumently on a weight-loss diet (No) } \\
\hline Yes & -24.35 & {$[-45.09,-3.61]$} & 0.02 & -9.29 & {$[-39.02,20.44]$} & 0.54 & 3.98 & {$[-9.81,17.77]$} & 0.57 \\
\hline
\end{tabular}


Table 2 (continued). Association between sociodemographic and lifestyle factors and plant-source food groups. Coefficient estimate; [ $95 \% \mathrm{Cl}]$; $\mathrm{p}$-value; bold: results with a p-value below or equal to $0.05(\mathrm{n}=2,057)$.

\begin{tabular}{|c|c|c|c|c|c|c|c|c|c|}
\hline \multirow{2}{*}{$\begin{array}{l}\text { Covariate (Reference) } \\
\text { Sex (Female) }\end{array}$} & \multicolumn{3}{|c|}{ Vegetables } & \multicolumn{3}{|c|}{ Nuts and seeds } & \multicolumn{3}{|c|}{ Oils and fats } \\
\hline & & & & & & & & & \\
\hline Male & -2.34 & {$[-12.26,7.58]$} & 0.64 & 0.87 & {$[-3.84,5.58]$} & 0.72 & 0.99 & {$[-0.84 ; 2.83]$} & 0.29 \\
\hline \multicolumn{10}{|l|}{ Nationality (Swiss) } \\
\hline European & -1.97 & {$[-12.8,8.86]$} & 0.72 & 4.60 & {$[-1.16,10.36]$} & 0.12 & 3.06 & {$[1.07 ; 5.05]$} & 0.00 \\
\hline $\begin{array}{l}\text { Region of the } \\
\text { Americas }\end{array}$ & 26.59 & {$[-33.51,86.68]$} & 0.39 & 5.68 & {$[-11.22,22.57]$} & 0.51 & 6.37 & {$[-3.48 ; 16.22]$} & 0.20 \\
\hline African / Eastem Mediterranean & -7.56 & {$[-52.5,37.39]$} & 0.74 & 6.92 & {$[-21.19,35.03]$} & 0.63 & 0.97 & {$[-7.18 ; 9.12]$} & 0.82 \\
\hline $\begin{array}{l}\text { Westem Pacific / } \\
\text { South-East Asia }\end{array}$ & 75.50 & {$[46.11,104.88]$} & 0.00 & 4.39 & {$[-16.03,24.81]$} & 0.67 & -2.45 & {$[-7.92 ; 3.01]$} & 0.38 \\
\hline \multicolumn{10}{|l|}{$\begin{array}{l}\text { Language region } \\
\text { (German-speaking) }\end{array}$} \\
\hline French-speaking & 11.66 & {$[1.43,21.9]$} & 0.03 & 2.55 & {$[-2.49,7.59]$} & 0.32 & 0.09 & {$[-1.78 ; 1.95]$} & 0.93 \\
\hline Italian-spea king & -12.03 & {$[-32.64,8.58]$} & 0.25 & -3.59 & {$[-14.84,7.67]$} & 0.53 & 8.78 & {$[5.15 ; 12.42]$} & 0.00 \\
\hline \multicolumn{10}{|l|}{ Age group (30-44 years) } \\
\hline $18-29$ years & 6.53 & {$[-9.31,22.37]$} & 0.42 & -0.64 & {$[-8.19,6.9]$} & 0.87 & 0.61 & {$[-2.08 ; 3.3]$} & 0.66 \\
\hline $45-59$ years & -1.36 & {$[-12.89,10.16]$} & 0.82 & 1.50 & {$[-4.32,7.32]$} & 0.61 & -0.27 & {$[-2.41 ; 1.87]$} & 0.80 \\
\hline $60-75$ years & 15.38 & {$[1.11,29.65]$} & 0.04 & -1.63 & {$[-8.78,5.51]$} & 0.65 & 2.36 & {$[-0.04 ; 4.77]$} & 0.06 \\
\hline \multicolumn{10}{|l|}{$\begin{array}{l}\text { Education; highest } \\
\text { degree (Secondary) }\end{array}$} \\
\hline Primary & 22.73 & {$[-2.9,48.36]$} & 0.08 & 1.62 & {$[-12.27,15.51]$} & 0.82 & 3.17 & {$[-1.73 ; 8.07]$} & 0.20 \\
\hline Tertiary & 16.72 & {$[7.44,25.99]$} & 0.00 & -0.72 & {$[-5.43,4]$} & 0.76 & -0.77 & {$[-2.48 ; 0.95]$} & 0.38 \\
\hline \multicolumn{10}{|l|}{ Civil status (Single) } \\
\hline Mamied & 0.68 & {$[-13.63,14.99]$} & 0.93 & 1.09 & {$[-5.92,8.11]$} & 0.76 & -1.77 & {$[-3.99 ; 0.45]$} & 0.12 \\
\hline Divorced & 12.30 & {$[-5,29.61]$} & 0.16 & 0.87 & {$[-7.67,9.41]$} & 0.84 & 1.88 & {$[-1.2 ; 4.96]$} & 0.23 \\
\hline Other & 1.60 & {$[-21.25,24.46]$} & 0.89 & 16.35 & {$[5.48,27.22]$} & 0.00 & 4.01 & {$[-0.3 ; 8.32]$} & 0.07 \\
\hline \multicolumn{10}{|l|}{ Income group $(6,000$} \\
\hline \multicolumn{10}{|l|}{ - $13,000 \mathrm{CHF} /$ month) } \\
\hline$\varangle 6,000 \mathrm{CHF} /$ month & -18.37 & {$[-29.78,-6.96]$} & 0.00 & -8.31 & {$[-13.94,-2.69]$} & 0.00 & -1.00 & {$[-3.05 ; 1.05]$} & 0.34 \\
\hline$>13,000 \mathrm{CHF} /$ month & 0.72 & {$[-10.42,11.86]$} & 0.90 & -1.08 & {$[-6.55,4.39]$} & 0.70 & 0.63 & {$[-1.47 ; 2.74]$} & 0.56 \\
\hline \multicolumn{10}{|l|}{ Smoking status (No) } \\
\hline Current & 4.01 & {$[-7.37,15.38]$} & 0.49 & -0.75 & {$[-6.83,5.33]$} & 0.81 & -1.50 & {$[-3.64 ; 0.63]$} & 0.17 \\
\hline Former & -3.43 & {$[-13.17,6.31]$} & 0.49 & -4.23 & {$[-8.68,0.23]$} & 0.06 & -0.25 & {$[-2.07 ; 1.57]$} & 0.79 \\
\hline \multicolumn{10}{|l|}{ BMI group (Nomal) } \\
\hline Underweight & 14.37 & {$[-17.98,46.73]$} & 0.38 & 29.39 & {$[16.62,42.17]$} & 0.00 & 1.26 & {$[-4.62 ; 7.15]$} & 0.67 \\
\hline Overweight & -10.41 & {$[-20.31,-0.51]$} & 0.04 & 0.59 & {$[-4.29,5.47]$} & 0.81 & -1.86 & {$[-3.72 ; 0.01]$} & 0.05 \\
\hline Obese & 4.50 & {$[-9.49,18.5]$} & 0.53 & 1.44 & {$[-6.83,9.7]$} & 0.73 & -0.80 & {$[-3.44 ; 1.85]$} & 0.56 \\
\hline \multicolumn{10}{|l|}{ Cumently on a weight-loss diet(No) } \\
\hline Yes & 29.30 & {$[9.08,49.51]$} & 0.01 & 1.69 & {$[-7.66,11.04]$} & 0.72 & 2.82 & {$[-0.97 ; 6.61]$} & 0.14 \\
\hline
\end{tabular}

While starchy food groups such as cereals and potatoes showed a positive association with being male $(+15.4 \mathrm{~g}$ and $+23.9 \mathrm{~g})$, consumption of fruits was lower among male participants compared to females (-32.5 g). Remarkable was also the difference in the consumption of alcoholic beverages, which was $146.3 \mathrm{~g}$ higher among male participants. Further, it was interesting to note the differences between language regions: while the participants from the French-speaking language region showed higher pulses (+9.8 g) and vegetables (+11.7 g) consumption and those of the Italianspeaking language region higher oils and fats consumption $(+8.8 \mathrm{~g})$, both of these language regions had a lower sugar consumption (-12.2 $\mathrm{g}$ and $-17.1 \mathrm{~g}$ ), as compared to the German-speaking language region. A similar pattern could be observed for participants older than the reference and those that completed tertiary education. Notably, participants with lower income than the reference groups revealed a negative association with the consumption of vegetables (-18.4 g), nuts and seeds (-8.3 $\mathrm{g})$, and alcoholic beverages $(-57.3 \mathrm{~g})$. Then, while fruits consumption was lower among current smokers $(-45.4 \mathrm{~g})$, consumption of alcoholic beverages was substantially higher both among current $(+140.3$ g) and former (+69.6 g) smokers, as compared to non-smokers. 
Table 2 (continued). Association between sociodemographic and lifestyle factors and plant-source food groups. Coefficient estimate; [95\% Cl]; p-value; bold: results with a p-value below or equal to $0.05(n=2,057)$.

\begin{tabular}{|c|c|c|c|c|c|c|c|c|c|}
\hline \multirow{2}{*}{$\begin{array}{l}\text { Covariate (Reference) } \\
\text { Sex (Female) }\end{array}$} & \multicolumn{3}{|l|}{ Fruits } & \multicolumn{3}{|l|}{ Sugar } & \multicolumn{3}{|c|}{ Alcoholic beverages } \\
\hline & & & & & & & & & \\
\hline Male & -32.52 & {$[-56.26,-8.77]$} & 0.01 & -4.58 & {$[-11.85,2.69]$} & 0.22 & 146.25 & {$[97.45,195.05]$} & 0.00 \\
\hline \multicolumn{10}{|l|}{ Nationality (Swiss) } \\
\hline European & 24.03 & {$[-2.05,50.11]$} & 0.07 & -7.32 & {$[-15.26,0.63]$} & 0.07 & 83.13 & {$[28.74,137.52]$} & 0.00 \\
\hline $\begin{array}{l}\text { Region of the } \\
\text { Americas }\end{array}$ & 26.63 & {$[-92.44,145.71]$} & 0.66 & 13.64 & {$[-24.23,51.51]$} & 0.48 & -19.27 & {$[-447.01,408.46]$} & 0.93 \\
\hline African / Eastem Mediterranean & 26.27 & {$[-95.77,148.31]$} & 0.67 & 7.86 & {$[-26.47,42.19]$} & 0.65 & -193.87 & {$[-486.34,98.6]$} & 0.19 \\
\hline $\begin{array}{l}\text { Westem Pacific / } \\
\text { South-East Asia }\end{array}$ & 4.57 & {$[-69.61,78.75]$} & 0.90 & 52.14 & {$[29.65,74.63]$} & 0.00 & 159.13 & {$[-27.73,346]$} & 0.10 \\
\hline \multicolumn{10}{|l|}{$\begin{array}{l}\text { Language region } \\
\text { (German-speaking) }\end{array}$} \\
\hline French-speaking & -6.64 & {$[-30.65,17.37]$} & 0.59 & -12.23 & {$[-19.68,-4.77]$} & 0.00 & 15.00 & {$[-35.5,65.5]$} & 0.56 \\
\hline Ita lian-spea king & 5.61 & {$[-44.77,55.98]$} & 0.83 & -17.14 & {$[-32.26,-2.01]$} & 0.03 & -55.50 & {$[-155.56,44.57]$} & 0.28 \\
\hline \multicolumn{10}{|l|}{ Age group (30-44 years) } \\
\hline $18-29$ years & -16.35 & {$[-53.49,20.78]$} & 0.39 & -8.84 & {$[-20.3,2.63]$} & 0.13 & 120.02 & {$[35.73,204.31]$} & 0.01 \\
\hline $45-59$ years & 54.77 & {$[26.67,82.86]$} & 0.00 & -14.55 & {$[-23.12,-5.98]$} & 0.00 & 27.14 & {$[-30.27,84.56]$} & 0.35 \\
\hline $60-75$ years & 72.60 & {$[38.35,106.84]$} & 0.00 & -22.64 & {$[-33.27,-12]$} & 0.00 & -31.35 & {$[-102.5,39.8]$} & 0.39 \\
\hline \multicolumn{10}{|l|}{$\begin{array}{l}\text { Education; highest } \\
\text { degree (Sec ondary) }\end{array}$} \\
\hline Primary & -3.24 & {$[-66.46,59.97]$} & 0.92 & 2.83 & {$[-16.23,21.9]$} & 0.77 & 54.63 & {$[-98.56,207.83]$} & 0.48 \\
\hline Tertiary & 26.81 & {$[4.67,48.95]$} & 0.02 & -9.53 & {$[-16.39,-2.67]$} & 0.01 & -43.41 & {$[-89.9,3.09]$} & 0.07 \\
\hline \multicolumn{10}{|l|}{ Civil status (Single) } \\
\hline Mamied & -17.70 & {$[-53.21,17.81]$} & 0.33 & -8.85 & {$[-19.63,1.94]$} & 0.11 & -24.71 & {$[-97.45,48.03]$} & 0.51 \\
\hline Divorced & -22.55 & {$[-64.36,19.26]$} & 0.29 & -4.32 & {$[-17.13,8.49]$} & 0.51 & 30.77 & {$[-55.54,117.07]$} & 0.48 \\
\hline Other & -46.98 & {$[-101.29,7.33]$} & 0.09 & -2.32 & {$[-19.28,14.63]$} & 0.79 & 85.45 & {$[-46.15,217.04]$} & 0.20 \\
\hline \multicolumn{10}{|l|}{ Income group $(6,000$} \\
\hline$<6,000 \mathrm{CHF} /$ month & -8.16 & {$[-35.7,19.38]$} & 0.56 & -3.90 & {$[-12.26,4.45]$} & 0.36 & -57.31 & {$[-114.78,0.17]$} & 0.05 \\
\hline$>13,000 \mathrm{CHF} /$ month & 9.49 & {$[-17.35,36.33]$} & 0.49 & -5.98 & {$[-14.32,2.37]$} & 0.16 & -24.44 & {$[-79.95,31.08]$} & 0.39 \\
\hline \multicolumn{10}{|l|}{ Smoking status (No) } \\
\hline Current & -45.38 & {$[-73.32,-17.44]$} & 0.00 & -1.21 & {$[-9.64,7.22]$} & 0.78 & 140.33 & [84.39,196.27] & 0.00 \\
\hline Former & 7.83 & {$[-15.28,30.93]$} & 0.51 & -7.65 & {$[-14.83,-0.47]$} & 0.04 & 69.63 & {$[20.41,118.84]$} & 0.01 \\
\hline \multicolumn{10}{|l|}{ BMI group (Nomal) } \\
\hline Underweight & 40.73 & {$[-35.79,117.24]$} & 0.30 & -2.18 & {$[-25.21,20.86]$} & 0.85 & -54.54 & {$[-217.54,108.45]$} & 0.51 \\
\hline Overweight & -33.08 & {$[-57.08,-9.09]$} & 0.01 & 4.39 & {$[-2.97,11.75]$} & 0.24 & -6.29 & {$[-54.87,42.29]$} & 0.80 \\
\hline Obese & -1.38 & {$[-34.83,32.07]$} & 0.94 & 9.48 & {$[-0.85,19.8]$} & 0.07 & 67.44 & {$[-3.2,138.08]$} & 0.06 \\
\hline \multicolumn{10}{|l|}{ Cumently on a weight-loss diet (No) } \\
\hline Yes & -19.45 & {$[-69.34,30.43]$} & 0.44 & -12.35 & {$[-27.57,2.88]$} & 0.11 & 16.39 & {$[-102.34,135.12]$} & 0.79 \\
\hline
\end{tabular}

\subsubsection{Sociodemographic and lifestyle factors and impacts}

The link between sociodemographic and lifestyle factors and impacts is clearly not a direct one, but is mediated by food choices and the related impacts per food groups. We also assessed this indirect linkage, to then better understand which sociodemographic and lifestyle aspects correlated with significant differences in impacts and which food choices are behind these patterns. We thus investigated sociodemographic and lifestyle variables for their associations with different impact categories that we calculated per dietary recall, and then combined the different analyses of this and the previous section in Section 3.2.3. Here, we first present results for the three environmental indicators GWP, CLO, and GLO, followed by the socio-economic indicators on social risks (SHI), diet quality (AHEI), and dietary cost. These indicators can be interpreted as follows: for GWP and LO, a higher value means higher emissions and higher land occupation respectively. For SHI, higher values indicate higher social risks along the food production chain, and for the AHEI, higher scores mean higher diet quality. Finally, from a consumer perspective, lower dietary cost are generally preferred. 
For GWP, the variables nationality, language region, age group, civil status, income group, smoking status, and being currently on a diet showed significant results, while for CLO, sex, nationality, language region, age group, and being currently on a diet showed significant results (Table 3). For CLO, the variables nationality, language region, civil status, income group, smoking status, BMI group, and being currently on a diet showed significant results. While GWP was higher for participants from the African / Eastern Mediterranean region, French-speaking language region, older than the reference, married, with a lower income than the reference, current smoker, and currently on a weight-loss diet, CLO was further positively associated with being male. Moreover, for GLO, participants from the European region and from the French-speaking language region showed a negative association, and being married, having an income above 13,000 CHF/month, being overweight, and being currently on a weight-loss diet showed a positive association.

Table 3. Association between sociodemographic and lifestyle factors and impact categories. Coefficient estimate; [95\% Cl]; $\mathrm{p}$-value; bold: results with a $\mathrm{p}$-value below or equal to $0.05(n=2,057)$.

\begin{tabular}{|c|c|c|c|c|c|c|c|c|c|}
\hline \multirow{2}{*}{$\begin{array}{l}\text { Covariate (Reference) } \\
\text { Sex (Female) }\end{array}$} & \multicolumn{3}{|c|}{ GWP (kg CO 2 eq) } & \multicolumn{3}{|c|}{ CLO $\left(\mathrm{m}^{2}\right)$} & \multicolumn{3}{|c|}{ GLO $\left(m^{2}\right)$} \\
\hline & & & & & & & & & \\
\hline Male & 0.01 & {$[-0.06 ; 0.09]$} & 0.77 & 0.10 & {$[0 ; 0.2]$} & 0.04 & -0.04 & {$[-0.1 ; 0.01]$} & 0.13 \\
\hline \multicolumn{10}{|l|}{ Nationality (Swiss) } \\
\hline European & 0.03 & {$[-0.06 ; 0.11]$} & 0.51 & 0.05 & {$[-0.06 ; 0.16]$} & 0.37 & -0.15 & {$[-0.22 ;-0.09]$} & 0.00 \\
\hline $\begin{array}{l}\text { Region of the } \\
\text { Americas }\end{array}$ & -0.19 & {$[-0.59 ; 0.21]$} & 0.36 & 0.32 & {$[-0.19 ; 0.83]$} & 0.22 & -0.14 & {$[-0.45 ; 0.17]$} & 0.36 \\
\hline Afric an / Eastem Meditemanean & 0.60 & {$[0.24 ; 0.96]$} & 0.00 & 0.71 & {$[0.25 ; 1.17]$} & 0.00 & 0.17 & {$[-0.1 ; 0.46]$} & 0.21 \\
\hline $\begin{array}{l}\text { Westem Pacific / } \\
\text { South-East Asia }\end{array}$ & 0.13 & {$[-0.11 ; 0.37]$} & 0.28 & 0.17 & {$[-0.14 ; 0.47]$} & 0.28 & -0.13 & {$[-0.32 ; 0.05]$} & 0.15 \\
\hline \multicolumn{10}{|l|}{$\begin{array}{l}\text { Language region } \\
\text { (Geman-speaking) }\end{array}$} \\
\hline French-speaking & 0.12 & {$[0.04 ; 0.2]$} & 0.00 & 0.16 & {$[0.06 ; 0.26]$} & 0.00 & -0.06 & {$[-0.12 ; 0]$} & 0.04 \\
\hline Ita lian-spea king & 0.13 & {$[-0.03 ; 0.29]$} & 0.11 & 0.18 & {$[-0.02 ; 0.38]$} & 0.07 & -0.09 & {$[-0.21 ; 0.03]$} & 0.13 \\
\hline \multicolumn{10}{|l|}{ Age group (30-44 years) } \\
\hline $18-29$ years & 0.02 & {$[-0.09 ; 0.13]$} & 0.71 & -0.01 & {$[-0.15 ; 0.13]$} & 0.92 & -0.03 & {$[-0.12 ; 0.05]$} & 0.46 \\
\hline $45-59$ years & 0.17 & {$[0.08 ; 0.26]$} & 0.00 & 0.21 & {$[0.1 ; 0.33]$} & 0.00 & 0.02 & {$[-0.04 ; 0.09]$} & 0.46 \\
\hline $60-75$ years & 0.26 & {$[0.16 ; 0.36]$} & 0.00 & 0.30 & {$[0.18 ; 0.43]$} & 0.00 & 0.05 & {$[-0.03 ; 0.13]$} & 0.19 \\
\hline \multicolumn{10}{|l|}{$\begin{array}{l}\text { Education; highest } \\
\text { degree (Secondary) }\end{array}$} \\
\hline Primary & 0.08 & {$[-0.12 ; 0.28]$} & 0.45 & -0.07 & {$[-0.32 ; 0.19]$} & 0.62 & 0.04 & {$[-0.12 ; 0.19]$} & 0.62 \\
\hline Tertiary & 0.05 & {$[-0.02 ; 0.12]$} & 0.17 & 0.01 & {$[-0.08 ; 0.1]$} & 0.87 & 0.03 & {$[-0.12 ; 0.19]$} & 0.67 \\
\hline \multicolumn{10}{|l|}{ Civil status (Single) } \\
\hline Mamied & 0.19 & {$[0.09 ; 0.28]$} & 0.00 & 0.07 & {$[-0.04 ; 0.19]$} & 0.22 & 0.13 & {$[0.06 ; 0.2]$} & 0.00 \\
\hline Divorced & -0.04 & {$[-0.17 ; 0.09]$} & 0.53 & -0.03 & {$[-0.2 ; 0.13]$} & 0.70 & -0.02 & {$[-0.12 ; 0.08]$} & 0.64 \\
\hline Other & -0.11 & {$[-0.28 ; 0.07]$} & 0.24 & 0.18 & {$[-0.05 ; 0.4]$} & 0.12 & -0.13 & {$[-0.27 ; 0]$} & 0.05 \\
\hline \multicolumn{10}{|l|}{$\begin{array}{l}\text { Income group }(6,000 \\
-13,000 \mathrm{CHF} / \text { month })\end{array}$} \\
\hline$<6,000 \mathrm{CHF} /$ month & 0.11 & {$[0.02 ; 0.19]$} & 0.01 & -0.04 & {$[-0.14 ; 0.07]$} & 0.52 & 0.00 & {$[-0.08 ; 0.06]$} & 0.77 \\
\hline$>13,000 \mathrm{CHF} /$ month & 0.03 & {$[-0.05 ; 0.12]$} & 0.48 & 0.02 & {$[-0.09 ; 0.13]$} & 0.75 & 0.08 & {$[0 ; 0.17]$} & 0.04 \\
\hline \multicolumn{10}{|l|}{ Smoking status (No) } \\
\hline Current & 0.09 & {$[0 ; 0.18]$} & 0.05 & 0.02 & {$[-0.09 ; 0.13]$} & 0.74 & -0.05 & {$[-0.13 ; 0.01]$} & 0.10 \\
\hline Former & 0.01 & {$[-0.07 ; 0.08]$} & 0.80 & -0.02 & {$[-0.12 ; 0.07]$} & 0.67 & -0.09 & {$[-0.17 ;-0.03]$} & 0.00 \\
\hline \multicolumn{10}{|l|}{ BMI group (Nomal) } \\
\hline Underweight & -0.08 & {$[-0.32 ; 0.16]$} & 0.52 & 0.01 & {$[-0.3 ; 0.32]$} & 0.96 & 0.00 & {$[-0.2 ; 0.18]$} & 0.91 \\
\hline Overweight & 0.07 & {$[-0.01 ; 0.15]$} & 0.08 & -0.02 & {$[-0.12 ; 0.07]$} & 0.62 & 0.09 & {$[0.04 ; 0.16]$} & 0.00 \\
\hline Obese & -0.09 & {$[-0.19 ; 0.02]$} & 0.12 & -0.01 & {$[-0.15 ; 0.13]$} & 0.92 & 0.12 & {$[-0.07 ; 0.1]$} & 0.72 \\
\hline \multicolumn{10}{|l|}{ Cumently on a weight-loss diet (No) } \\
\hline Yes & 0.31 & {$[0.15 ; 0.47]$} & 0.00 & 0.28 & {$[0.07 ; 0.48]$} & 0.01 & 0.12 & {$[0 ; 0.25]$} & 0.05 \\
\hline
\end{tabular}


Table 3 (continued). Association between sociodemographic and lifestyle factors and impact categories. Coefficient estimate; [95\% Cl]; p-value; bold: results with a p-value below or equal to $0.05(n=2,057)$.

\begin{tabular}{|c|c|c|c|c|c|c|c|c|c|}
\hline \multirow{2}{*}{$\begin{array}{l}\text { Covariate (Reference) } \\
\text { Sex (Female) }\end{array}$} & \multicolumn{3}{|c|}{ SHII (Index) } & \multicolumn{3}{|c|}{ AHEl (Index) } & \multicolumn{3}{|c|}{ Cost (CHF) } \\
\hline & & & & & & & & & \\
\hline Male & -0.06 & {$[-0.14 ; 0.01]$} & 0.09 & -1.32 & {$[-2.66 ; 0.01]$} & 0.05 & 1.37 & {$[0.65 ; 2.08]$} & 0.00 \\
\hline \multicolumn{10}{|l|}{ Nationality (Swiss) } \\
\hline European & 0.07 & {$[-0.01 ; 0.16]$} & 0.07 & 1.55 & {$[0.08 ; 3.01]$} & 0.04 & 1.66 & {$[0.88 ; 2.44]$} & 0.00 \\
\hline $\begin{array}{l}\text { Region of the } \\
\text { Americas }\end{array}$ & 0.33 & {$[-0.05 ; 0.71]$} & 0.09 & 5.63 & {$[-1.41 ; 12.67]$} & 0.12 & -1.99 & {$[-5.75 ; 1.76]$} & 0.30 \\
\hline African / Eastem Meditemanean & -0.26 & {$[-0.60 ; 0.08]$} & 0.14 & -0.46 & {$[-6.82 ; 5.91]$} & 0.89 & 2.31 & {$[-1.09 ; 5.7]$} & 0.18 \\
\hline $\begin{array}{l}\text { Westem Pacific / } \\
\text { South-East Asia }\end{array}$ & 0.02 & {$[-0.21 ; 0.24]$} & 0.87 & 8.38 & [4.18;12.57] & 0.00 & 5.10 & {$[2.87 ; 7.33]$} & 0.00 \\
\hline \multicolumn{10}{|l|}{$\begin{array}{l}\text { Language region } \\
\text { (German-speaking) }\end{array}$} \\
\hline French-speaking & 0.04 & {$[-0.03 ; 0.12]$} & 0.28 & 1.65 & {$[0.27 ; 3.02]$} & 0.02 & 1.49 & {$[0.76 ; 2.22]$} & 0.00 \\
\hline Italian-spea king & -0.24 & {$[-0.39 ;-0.09]$} & 0.00 & 2.73 & {$[-0.03 ; 5.49]$} & 0.05 & 0.78 & {$[-0.69 ; 2.25]$} & 0.30 \\
\hline \multicolumn{10}{|l|}{ Age group (30-44 yea rs) } \\
\hline $18-29$ years & -0.27 & {$[-0.37 ;-0.16]$} & 0.00 & -0.56 & {$[-2.52 ; 1.40]$} & 0.57 & 0.68 & {$[-0.36 ; 1.73]$} & 0.20 \\
\hline $45-59$ years & 0.11 & {$[0.02 ; 0.19]$} & 0.01 & 3.56 & {$[1.98 ; 5.13]$} & 0.00 & 1.55 & {$[0.72 ; 2.39]$} & 0.00 \\
\hline $60-75$ years & 0.22 & {$[0.12 ; 0.31]$} & 0.00 & 6.43 & {$[4.67 ; 8.19]$} & 0.00 & 1.59 & {$[0.65 ; 2.53]$} & 0.00 \\
\hline \multicolumn{10}{|l|}{$\begin{array}{l}\text { Educ ation; highest } \\
\text { degree (Sec ondary) }\end{array}$} \\
\hline Primary & 0.04 & {$[-0.14 ; 0.23]$} & 0.66 & -0.83 & {$[-4.36 ; 2.71]$} & 0.65 & -0.27 & {$[-2.16 ; 1.61]$} & 0.78 \\
\hline Tertiary & 0.05 & {$[-0.01 ; 0.12]$} & 0.15 & 2.49 & {$[1.24 ; 3.733]$} & 0.00 & 0.14 & {$[-0.53 ; 0.8]$} & 0.69 \\
\hline \multicolumn{10}{|l|}{ Civil status (Single) } \\
\hline Mamied & -0.02 & {$[-0.11 ; 0.06]$} & 0.65 & -0.47 & {$[-2.09 ; 1.14]$} & 0.57 & 0.36 & {$[-0.49 ; 1.22]$} & 0.40 \\
\hline Divorced & 0.01 & {$[-0.11 ; 0.13]$} & 0.83 & -0.36 & {$[-2.63 ; 1.91]$} & 0.76 & 0.31 & {$[-0.9 ; 1.52]$} & 0.61 \\
\hline Other & -0.08 & {$[-0.24 ; 0.09]$} & 0.36 & -0.19 & {$[-3.30 ; 2.92]$} & 0.90 & -0.30 & {$[-1.95 ; 1.36]$} & 0.72 \\
\hline \multicolumn{10}{|l|}{ Income group $(6,000$} \\
\hline \multicolumn{10}{|l|}{ - 13,000 CHF/month) } \\
\hline$\varangle 6,000 \mathrm{CHF} /$ month & -0.04 & {$[-0.12 ; 0.05]$} & 0.40 & -0.23 & {$[-1.73 ; 1.27]$} & 0.76 & -0.56 & {$[-1.36 ; 0.24]$} & 0.17 \\
\hline$>13,000 \mathrm{CHF} /$ month & -0.03 & {$[-0.11 ; 0.05]$} & 0.48 & -0.01 & {$[-1.52 ; 1.51]$} & 0.99 & 0.21 & {$[-0.6 ; 1.02]$} & 0.61 \\
\hline \multicolumn{10}{|l|}{ Smoking status $(\mathrm{No})$} \\
\hline Current & -0.05 & {$[-0.14 ; 0.03]$} & 0.21 & -3.12 & {$[-4.67 ;-1.56]$} & 0.00 & 2.60 & [1.77;3.43] & 0.00 \\
\hline Former & 0.03 & {$[-0.04 ; 0.10]$} & 0.38 & -1.26 & {$[-2.58 ; 0.07]$} & 0.06 & 0.98 & {$[0.28 ; 1.69]$} & 0.01 \\
\hline \multicolumn{10}{|l|}{ BMI group (Nomal) } \\
\hline Underweight & 0.00 & {$[-0.23 ; 0.23]$} & 0.99 & 3.95 & {$[-0.31 ; 8.22]$} & 0.07 & -0.53 & {$[-2.81 ; 1.74]$} & 0.65 \\
\hline Overweight & -0.14 & {$[-0.21 ;-0.06]$} & 0.00 & -2.98 & {$[-4.34 ;-1.62]$} & 0.00 & 0.16 & {$[-0.56 ; 0.89]$} & 0.66 \\
\hline Obese & -0.03 & {$[-0.14 ; 0.07]$} & 0.51 & -5.00 & {$[-6.91 ;-3.09]$} & 0.00 & -0.54 & {$[-1.56 ; 0.47]$} & 0.29 \\
\hline \multicolumn{10}{|l|}{ Cumently on a weight-loss diet(No) } \\
\hline Yes & -0.07 & {$[-0.23 ; 0.08]$} & 0.35 & 4.24 & {$[1.45 ; 7.041]$} & 0.00 & 1.55 & {$[0.06 ; 3.05]$} & 0.04 \\
\hline
\end{tabular}

For the indicator $\mathrm{SHI}$, the variables language region, age group, and BMI group revealed significant results, while for AHEI, all variables except civil status, and income were significant. For dietary cost, all variables except education, civil status, income group, and BMI group showed significant results (see Table 3). Notably, being male was negatively associated with the AHEI (-1.3 points) and positively with cost $(+1.4 \mathrm{CHF})$. Further, participants from the European region ( +1.6 points) and from the Western Pacific and South-East Asia region (+8.4 points) revealed a higher AHEI, but also higher cost $(+1.7 \mathrm{CHF}$ and $+5.1 \mathrm{CHF})$. The SHI of participants from the Italian-speaking region was lower compared to the German-speaking region (-0.2 points), while their AHEI was higher (+2.7 points). Aligned with this latter result, AHEI was also higher for participants from the Frenchspeaking region (+1.7 points), as were cost $(+1.5 \mathrm{CHF})$. Differences between age groups were remarkable for these three indicator categories. While being younger than the reference group was associated with lower social risks (-0.3 points), being older than the reference group was on the one hand associated with higher social risks ( $45-59$ years: +0.1 points, $60-75$ years: +0.2 points) and higher cost $(+1.6 \mathrm{CHF})$, but on the other hand, also with a higher diet quality (45-59 years: +3.6 points, 60 - 
75 years: +6.4 points). In contrast to this, smoking was negatively associated with diet quality $(-3.1$ points), as was being overweight or obese ( -3.0 points and -5.0 points).

\subsubsection{Integrating sociodemographic and lifestyle factors, food choices, and impacts}

In this section, we combine the patterns observed between sociodemographic and lifestyle characteristics, food groups, and impacts into an encompassing picture. We start with a reading guidance for Figure 3 and then present the results.

Figure 3 can be interpreted as follows: for GWP, eight sociodemographic and lifestyle factors showed significant results (see Table 3), and were therefore included in the subsequent integrated analysis (see rows in Figure 3). In Figure 3, the coefficient estimate for GWP for these levels of variables is given in the third column (e.g. French-speaking language region was positively associated with GWP (+0.12 $\mathrm{kg} \mathrm{CO} 2 \mathrm{eq})$ ), followed by information on the direction of association between the respective variable and food groups (e.g. French-speaking language region was positively associated with cattle meat, chicken, pulses, and vegetables, and negatively associated with milk products and sugar, other sweeteners). Where the directions of the impact and of the food group were aligned, the squares are marked with an $\mathrm{x}$. We thus see that the French-speaking language region was positively associated both with GWP and with cattle meat, chicken, pulses, and vegetables. Moreover, the strength of the indicator (e.g. whether GWP of cattle meat was above or below the median of GWP, cf. Figure 2) is indicated with strong and weak (see colour legend of Figure 3). In summary, the increase in GWP of participants from the French-speaking language region was aligned with an increased consumption of cattle meat, chicken, pulses, and vegetables, where cattle meat and chicken show higher impact intensities for GWP.

Further results for GWP showed that for married participants, consumption of cattle meat and milk products can serve as explanation for the higher GWP. Increased CLO was significantly associated with six levels of variables (Figure 3). For male participants, cattle meat and chicken as well as pulses showed increased consumption levels and above median impact intensities. The same pattern applied for participants from the French-speaking language region. Higher consumption of cattle meat was furthermore likely to drive increased CLO levels of participants originating from the African / Eastern Mediterranean region. The two age groups above the reference group and the group of people being currently on a weight-loss diet showed higher CLO values, but no increase in food groups that have an above median impact intensity. There, increased consumption of fruits and vegetables (both with below median impact intensities) could contribute to increased CLO levels. Moreover, as grass resources are only fed to ruminants, only the two food groups cattle meat and milk products were relevant for this impact category. Reduced milk consumption was found in combination with lower GLO for European and French-speaking participants, as well as former smokers. On the contrary, being married was positively associated with cattle meat consumption, and also came along with a higher GLO.

For the SHI, five levels of variables showed significant results (Figure 3). Notably, participants from the Italian-speaking language region performed better on the SHI score, and at the same time revealed a lower intake of sugar and other sweeteners as well as milk products. Further, also 
younger participants revealed a lower SHI score, and older participants a higher. The latter result coincided with an increased consumption of vegetables and fruits, while the reduced consumption of sugar pointed into the other direction. Then, also overweight participants showed a lower SHI, which came along with a lower fruits and vegetables consumption.

For male participants, the lower score of the AHEI compared to female was aligned with a lower consumption of fruits and milk products (Figure 3). Further, the higher AHEI score for the European region compared to Swiss individuals was aligned with higher fish and seafood and eggs consumption, and for Western Pacific / South-East Asia region with higher vegetables, cattle meat, and sugar consumption. Both language regions French-speaking and Italian-speaking had a higher AHEI than the German-speaking reference, which coincided with an increased pulses and vegetables consumption (for the French-speaking language region) and a lower sugar consumption. The higher AHEI score of participants older than the reference group coincided with a higher fruits and vegetables consumption, which was also found for people with tertiary education. Further, the lower AHEI for current smokers was associated with lower fruits consumption, and the lower AHEI of overweight participants was associated with a lower consumption of cereals (whole grain), vegetables, and fruits. Finally, the higher AHEI score of people currently on a weight-loss diet also came with a higher vegetables consumption.

Cost of consumed foods were higher among male participants, coinciding with an increased consumption (and above median impact intensity) of cattle meat, chicken, and alcoholic beverages. Higher cost could also be observed among non-Swiss nationalities, coinciding with a higher intake of fish and seafood and alcoholic beverages (European region), and cattle meat and vegetables (Western Pacific / South-East Asia region) (Figure 3). Also for participants from the French-speaking language region, cattle meat and chicken as well as vegetables consumption, and pulses with below median impact intensity, were found in combination with increased cost. Further, for participants older than the reference group, fruits and vegetables coincided with increased cost, while for current and former smokers, cattle meat and alcoholic beverages were found in combination with higher cost. Finally, higher vegetables consumption coincided with higher cost for people being currently on a weight-loss diet.

Interestingly, the reduced consumption of the food group sugar and other sweeteners revealed synergies only, due to the fact that its consumption does not contribute to a positive effect of any impact category. Moreover, lower sugar consumption - as could for example be identified for participants from the French-speaking and Italian-speaking language region, older than the reference, and with tertiary education - could have contributed to weaken the negative impacts e.g. in form of higher GWP than the respective reference. Further, pork was an interesting case, as its consumption evidently did not differ between any sociodemographic and lifestyle factor that showed significant results for an impact category. However, since pork contributes with above median impact intensity to multiple impact categories (GWP, LO, and cost), and moreover does not contribute to an increase of the AHEI, we can still conclude that reduced consumption thereof is desirable. 


\begin{tabular}{|c|c|c|c|c|c|c|c|c|c|c|c|c|c|c|c|c|}
\hline Covariates & Reference & Impac & & & & & & & od & gr & roup & & & & & \\
\hline & & 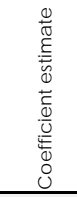 & 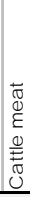 & $\begin{array}{l} \\
\text { ․ㅡㅇ } \\
\end{array}$ & 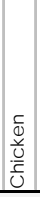 & $\begin{array}{l}y \\
y \\
\underline{z} \\
0 \\
0 \\
0 \\
\underline{z} \\
\underline{\underline{z}} \\
\end{array}$ & $\begin{array}{l} \\
\text { 员 } \\
\text { 号 } \\
\end{array}$ & 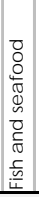 & 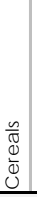 & $\begin{array}{l}n \\
0 \\
0 \\
\\
0 \\
0 \\
0\end{array}$ & 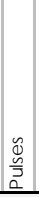 & 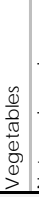 & & 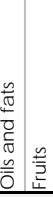 & & 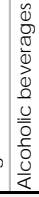 \\
\hline Global wa ming potential GWP ( $\mathrm{kg} \mathrm{CO}_{2}$ eq) & & & & & & & & & & & & & & & & \\
\hline Nationality: Afric an / Eastem Mediterranean & (Swiss) & 0.60 & $\mathbf{x}$ & & & & & & & $\mathbf{x}$ & & & & & & \\
\hline Language region: French-speaking & (German-speaking) & 0.12 & $\mathbf{x}$ & & $\mathbf{x}$ & & & & & & $\mathbf{x}$ & $\mathbf{x}$ & & & & \\
\hline Age group: $45-59$ years & (30-44 years) & 0.17 & & & & & & & & & & & & $\mathbf{x}$ & $x$ & \\
\hline Age group: $60-75$ years & (30-44 years) & 0.26 & & & & & & & & & & $\mathbf{x}$ & & $\mathbf{x}$ & $x$ & \\
\hline Civil status M a ried & (Single) & 0.19 & $\mathbf{x}$ & & & $\mathbf{x}$ & & & & & & & & & & \\
\hline Income group: $\varangle 6,000 \mathrm{CHF} /$ month & (6,000 - 13,000 CHF/month) & 0.11 & & & & & & & & $\mathbf{x}$ & & & & & & \\
\hline Smoking status Current & (No smoker) & 0.09 & $\mathbf{x}$ & & & & & & & & & & & & & $\mathbf{x}$ \\
\hline Cumenty on a weight-loss diet Yes & (No) & 0.31 & & & & & & & & & & $\mathbf{x}$ & & & & \\
\hline Cropland occupation CLO $\left(\mathrm{m}^{2}\right)$ & & & & & & & & & & & & & & & & \\
\hline Sex: Male & (Female) & 0.10 & $\mathbf{x}$ & & $\mathbf{x}$ & & & & $\mathbf{x}$ & $\mathbf{x}$ & $\mathbf{x}$ & & & & & $\mathbf{x}$ \\
\hline Nationality: Afric an / Eastem M editerranean & (Swiss) & 0.71 & $\mathbf{x}$ & & & & & & & $\mathbf{x}$ & & & & & & \\
\hline Language region: French-speaking & (German-speaking) & 0.16 & $\mathbf{x}$ & & $\mathbf{x}$ & & & & & & $\mathbf{x}$ & $\mathbf{x}$ & & & & \\
\hline Age group: $45-59$ years & (30-44 years) & 0.21 & & & & & & & & & & & & & $x$ & \\
\hline Age group: $60-75$ years & (30-44 years) & 0.30 & & & & & & & & & & $\mathbf{x}$ & & & $x$ & \\
\hline Cumenty on a weight-loss diet Yes & (No) & 0.28 & & & & & & & & & & $\mathbf{x}$ & & & & \\
\hline Grastand occupation GLO $\left(\mathrm{m}^{2}\right)$ & & & & & & & & & & & & & & & & \\
\hline Nationality: European & (Sw iss) & -0.15 & & & & $\mathbf{x}$ & & & & & & & & & & \\
\hline Language region: French-speaking & (German-speaking) & -0.06 & & & & $\mathbf{x}$ & & & & & & & & & & \\
\hline Civil status: Ma aried & (Single) & 0.13 & $\mathbf{x}$ & & & $\mathbf{x}$ & & & & & & & & & & \\
\hline Inc ome group: $>13,000 \mathrm{CHF} /$ month & (6,000 - 13,000 CHF/month) & 0.08 & & & & & & & & & & & & & & \\
\hline Smoking status Former & (No smoker) & -0.09 & & & & $\mathrm{x}$ & & & & & & & & & & \\
\hline BMI group: Overweight & (Normal) & 0.09 & & & & & & & & & & & & & & \\
\hline Cumently on a weight-loss diet Yes & (No) & 0.12 & & & & & & & & & & & & & & \\
\hline Social Hotspots Index SHII (Index) & & & & & & & & & & & & & & & & \\
\hline Language region: Italian-speaking & (German-speaking) & -0.24 & & & & $\mathbf{x}$ & & & & & & & & & $\mathbf{x}$ & \\
\hline Age group: $18-29$ years & (30-44 years) & -0.27 & & & & & & & & & & & & & & \\
\hline Age group: $45-59$ years & (30-44 years) & 0.11 & & & & & & & & & & & & & $x$ & \\
\hline Age group: $60-75$ years & (30-44 years) & 0.22 & & & & & & & & & & $\mathbf{x}$ & & & $x$ & \\
\hline BMI group: Overweight & (Normal) & -0.14 & & & & & & & $\mathbf{x}$ & & & $\mathbf{x}$ & & & $x$ & \\
\hline Altemate Healthy Eating Index AHE (Index) & & & & & & & & & & & & & & & & \\
\hline Sex: Male & (Female) & -1.32 & & & & $\mathbf{x}$ & & & & & & & & & $x$ & \\
\hline Nationality: European & (Sw iss) & 1.55 & & & & & $\mathbf{x}$ & $\mathbf{x}$ & & & & & & $\mathbf{x}$ & & $\mathbf{x}$ \\
\hline Nationality: Westem Pacific / South-East Asia & (Swiss) & 8.38 & $\mathbf{x}$ & & & & & & & & & $\mathbf{x}$ & & & $\mathbf{x}$ & \\
\hline Language region: French-speaking & (German-speaking) & 1.65 & $\mathbf{x}$ & & $\mathbf{x}$ & & & & & & $\mathbf{x}$ & $\mathbf{x}$ & & & & \\
\hline Language region: Italian-speaking & (German-speaking) & 2.73 & & & & & & & & & & & & $\mathbf{x}$ & & \\
\hline Age group: $45-59$ years & (30-44 years) & 3.56 & & & & & & & & & & & & & $x$ & \\
\hline Age group: $60-75$ years & (30-44 years) & 6.43 & & & & & & & & & & $\mathbf{x}$ & & & $x$ & \\
\hline Education: Tertiary & (Secondary) & 2.49 & & & & & & & & & & $\mathbf{x}$ & & & $x$ & \\
\hline Smoking status Current & (No smoker) & -3.12 & & & $\mathbf{x}$ & & & & & & & & & & $x$ & \\
\hline BMI group: Overweight & (Normal) & -2.98 & & & & & & & $\mathbf{x}$ & & & $\mathbf{x}$ & & & $\bar{x}$ & \\
\hline BMI group: Obese & (Normal) & -5.00 & & & & & & & & & & & & & & \\
\hline Currently on a weight-loss diet Yes & (No) & 4.24 & & & & & & & & & & $\mathbf{x}$ & & & & \\
\hline Cost(CHF) & & & & & & & & & & & & & & & & \\
\hline Sex: Male & (Female) & 1.37 & $\mathbf{x}$ & & $\mathbf{x}$ & & & & $\mathbf{x}$ & $\mathbf{x}$ & $\mathbf{x}$ & & & & & $\mathbf{x}$ \\
\hline Nationality: European & (Swiss) & 1.66 & & & & & $\mathbf{x}$ & $\mathbf{x}$ & & & & & & $\mathbf{x}$ & & $\mathbf{x}$ \\
\hline Nationality: Westem Pacific / South-East Asia & (Sw iss) & 5.10 & $\mathbf{x}$ & & & & & & & & & $\mathbf{x}$ & & & $\mathbf{x}$ & \\
\hline Language region: French-speaking & (German-speaking) & 1.49 & $\mathbf{x}$ & & $\mathbf{x}$ & & & & & & $\mathbf{x}$ & $\mathbf{x}$ & & & & \\
\hline Age group: $45-59$ years & (30-44 years) & 1.55 & & & & & & & & & & & & & $x$ & \\
\hline Age group: $60-75$ years & (30-44 years) & 1.59 & & & & & & & & & & $\mathbf{x}$ & & & $x$ & \\
\hline Smoking status Current & (No smoker) & 2.60 & $\mathbf{x}$ & & & & & & & & & & & & & $\mathbf{x}$ \\
\hline Smoking status Former & (No smoker) & 0.98 & & & & & & & & & & & & & & $\mathbf{x}$ \\
\hline Currently on a weight-loss diet Yes & (No) & 1.55 & & & & & & & & & & $\mathbf{x}$ & & & & \\
\hline & & & & & & & & & & & & & $\begin{array}{l}\text {, stron } \\
\text {, weak } \\
\text { haligne }\end{array}$ & & & \\
\hline
\end{tabular}

Figure 3. Association of sociodemographic and lifestyle factors and different environmental and socio-economic impact categories (coefficient estimate) $(n=2,057)$. The colour legend for the squares indicates the direction of the association between the sociodemographic and lifestyle factors and the food groups (yellow: positive; blue: negative; grey: non-significant/no impact), and the strength of the impact intensity of the respective food group (strong (dark colour) indicates an impact intensity above median, weak (light colour) indicates an impact intensity below median per impact category). Squares with aligned direction for the impact and food group are labelled with " $x$ ". 
In fact, we note that levels of sociodemographic and lifestyle factors that did not reveal significant results for the sustainability impacts calculated can still improve their performance by reducing the consumption of those food groups with above median impact intensities in particular. As an example, male participants do not reveal significant differences in GWP compared to females. This came along with reduced consumption of milk products and fruits, which could offset the effect of increased consumption of cattle meat, chicken, cereals, potatoes, pulses, and alcoholic beverages (Tables 1 and 2). Thus, although no difference could be observed between the two levels of the variable sex, adverse impacts could be mitigated by reducing the consumption of the named food groups. Notably, the same logic applies to all respective reference groups per impact category.

\subsection{Sustainability impacts, food choices, and population characteristics}

An increasing body of literature has combined diet quality with environmental, and partly economic, impacts in integrated assessments of different diets - either in scenario analyses (Chen et al., 2019; Tilman and Clark, 2014), or by assessing dietary recalls (Mertens et al., 2019; Walker et al., 2018). Mertens et al. (2019) recently attempted a comparison of GWP and LO of dietary recalls in four European countries, finding that GWP ranges from $5.1 \mathrm{~kg} \mathrm{CO} 2 \mathrm{eq}$ (median in Italy) to $5.9 \mathrm{~kg}$ $\mathrm{CO}_{2} \mathrm{eq}$ (median in France) per day, and total land use ranges from $6.6 \mathrm{~m}^{2}$ (median in Italy) to $7.3 \mathrm{~m}^{2}$ (France median in) per day. Previous estimates of the GWP for these countries found lower values, with $3.4 \mathrm{~kg} \mathrm{CO}$ eq for Italy (mean; Germani et al. (2014)), and $4.1 \mathrm{~kg} \mathrm{CO}$ eq for France (mean; Vieux et al. (2012)). According to recent estimates for Switzerland by Ernstoff et al. (2020), GWP of Swiss diets ranges between 3.0 and $7.1 \mathrm{~kg} \mathrm{CO}$ eq per capita per day. With $3.3 \mathrm{~kg} \mathrm{CO}$ eq for GWP and 6.4 $\mathrm{m}^{2}$ for total land use, our estimates are at the lower end of the ranges found in previous studies, but within the same order of magnitude. This difference can be traced to regional differences as well as differences in environmental impact assessment methods, such as system boundaries and assumed management practices (Frehner et al., 2020; Notarnicola et al., 2017).

Further, in most of these studies, reduction of ASF - in particular red meat - is identified to offer synergies for improvements of multiple environmental and human health impact categories, while increases of plant-source food, such as legumes, nuts, vegetables, and fruits, offer synergies for human health and, partly, environmental impact categories. Our results confirm these trends. Moreover, by expanding the impact categories towards including social risks and dietary cost, we show that when these two impact categories are included, plant-source food such as vegetables and fruits reveal trade-offs. In fact, both cost and SHI impact intensities are relatively high for these two food groups, resulting in substantial contributions to total SHI and cost. For the other impact categories, however, these food groups perform favourable, resulting in a trade-off between higher SHI and cost on the one hand, and improved AHEI and lower GWP and LO on the other hand. Thereby, it is important to highlight that these higher SHI scores relate to risks in labour conditions in import countries (e.g. in worker health and security). Chen et al. (2019) found that the cost for an average Swiss diet lies at around 7 CHF per capita per day, which is in the same order of magnitude as our cost estimate (9.3 CHF per capita per day).

Based on the menuCH dataset employed here, previous studies have shown that food consumption differs substantially between population subgroups, and in particular between the three language 
regions of Switzerland (Chatelan et al., 2017a). Pestoni et al. (2019) calculated a diet quality score (AHEI) for the menuCH recall data which enables comparisons between different diets, and was therefore also applied here to represent the human health dimension of the impacts. Higher AHEI scores were associated with being female, non-Swiss, originating from the French- or Italianspeaking part of Switzerland, being older, having obtained a tertiary education, being a non-smoker, and being normal weight. The food groups fish and seafood, cereals (whole grains), pulses, vegetables, and fruits mainly contribute to the good performance of these population subgroups. Further, we found that impact intensities of the two environmental indicators GWP and CLO were mostly aligned, meaning that food groups showing high impact intensities in GWP also showed high impact intensities in CLO. These results are in line with results from Walker et al. (2018). Also, population subgroups performing well were fairly aligned for these two impact categories; being female, Swiss, from the German-speaking part of Switzerland, and 30-44 years revealed lower GWP and CLO levels. These results can mainly be explained by lower intake of cattle meat, chicken, and to a lesser extent of different plant-source food. The impact category cost revealed a similar pattern to the two environmental indicators GWP and CLO for ASF, i.e. that mainly cattle meat and chicken contributed to increased cost levels of similar population subgroups. However, for cost, also vegetables and fruits consumption contributed to this effect. For the SHI, the contribution of fruits and vegetables was even more pronounced.

\subsection{Limitations and implications}

We also want to point out some limitations of the study. First, although we covered indicators of multiple dimensions of sustainability, the chosen indicators only represent a part of total sustainability. The results of this study therefore depend on the selected indicators, and might look different if different indicators would have been employed. A prominent example for this is the case of pasture-based cattle meat production versus chicken: while the cattle meat production comes with relatively high GHG emissions, it disposes over the advantage of being able to convert grass resources, which otherwise could hardly be used for human food production, into valuable animalsource food (Schader et al., 2015; Van Zanten et al., 2019). By this latter advantage, the cropland occupation is accordingly minimised. This example shows that if only GHG emissions and cropland occupation are considered, information on the suitability of resources and the associated feed-food competition is lost. Thus, the performance of single food products is always dependent on the choice of indicators, which should be born in mind when interpreting our results. There are many other areas of concern regarding environmental impacts of food production, such as marine and terrestrial biodiversity loss, soil degradation, and others (Campbell et al., 2017).

Second, as total food intake per person is limited, food groups that can serve as substitutes for each other are consequently mutually limiting. However, the current analysis did not impose limitations per food group. Within the scope of our study, as no inference is drawn based on the absolute level of the coefficient estimates for food groups, neglecting these mutual dependencies can be accepted. It has to be noted, however, that as soon as absolute levels of the coefficient estimates or interrelations between food groups are investigated further, these restrictions need to be considered. Examples for how this could be accounted for can be found e.g. in literature dealing with almost 
ideal demand systems (Deaton and Muellbauer, 1980). Lokuge et al. (2019), for example, propose a quadratic almost ideal demand system, which enables consumer expenditure allocation with limited total budgets. In fact, by employing price and expenditure elasticities, resources are allocated in a realistic manner between substitutes, thus solving the problem of unlimited coefficient estimates.

Third, data used and indicators assessed come with limitations. Dietary recall data, as were employed here, face for example the issue of potential underreporting (Gemming and Mhurchu, 2016). Del Gobbo et al. (2015) compared national estimates from the FAO with dietary recall, and found substantially higher values in FAO estimates than dietary recall estimates for the food groups vegetables, whole grains, and lower values for beans and legumes, and nuts and seeds. Moreover, the SHI has to be interpreted with care; this indicator is based on country-level data and thus provides an indication on where social risks, such as poor working conditions, currently exist and may play a role when sourcing food from these countries. It is thus not a statement on whether for a particular food the working conditions and other social aspects are in fact bad or not, but rather a statement on the risk that this may be the case. Thus, results for the SHI should serve as indication that undesirable risks in producer countries could occur, and these risks then need to be appropriately considered.

It is important to consider that the indicators assessed cover a broad range of topics but also are of different quality regarding the conclusions that can be drawn. For GWP, actions regarding changes in consumption volumes of certain food groups (e.g. cattle meat) can directly be related to corresponding reductions or increases in emissions and thus climate change mitigation effects. For the AHEI, this relation between indicator and targeted effect (human health) is less direct, and a change in food groups and corresponding changes in AHEI may only give some indication that the chance to improve health status may increase or decrease. For the SHI, these relations are even more indirect, as the SHI stands for a risk that certain social impacts may occur for certain food groups with higher or lower probability, depending on where they are sourced from. Thus, while for measures to reduce GHG emissions, direct recommendations for changes in dietary composition can be given (e.g. less cattle meat and other animal source food), for the SHI suggestions for action read differently. Instead of avoiding the consumption of the respective food items that come with a higher risk of adverse social impacts, actors should focus on how the production process, and in particular working conditions, can be improved for the food groups and countries of origin in question.

\section{Conclusion}

The impacts of our food choices are manifold, ranging from environmental and social impacts at the food production stage to dietary quality and cost at the consumption stage. Our results showed that any action on food groups, be it for health, social or environmental reasons, potentially affects societal groups differently. The analysis showed that, for example, a $\mathrm{CO}_{2}$-tax on cattle meat would disproportionally affect other than Swiss nationalities, while it would however not specifically affect low or high-income groups. Another example would be a measure on foods with relatively high social risks, which would disproportionally affect people older than the reference group. Overall, we can conclude that nationalities, language regions, age groups, and smoking status seemed 
particularly distinctive, while income or educational groups seemed hardly relevant when looking at combined effects of food choices and impacts. This is of particular relevance, as there was no indication that measures to curb the various impacts would predominantly disadvantage lower income groups. Furthermore, sex, civil status, BMI group, and being currently on a weight-loss diet were partly relevant. This provides important information on how to target measures to support more sustainable, healthy, and social diets, and which aspects to pay particular attention to and which are less central. We moreover showed that reductions of some food groups, especially different types of meat, offer large potentials for synergies on multiple impact categories. Others, such as fruits and vegetables as well as fish and seafood, resulted in trade-offs, by contributing to an improved performance of diet quality on the one hand, but on the other hand being rather costly and, for fruits and vegetables, additionally posing social risks.

Furthermore, we provided new insights on how sociodemographic and lifestyle factors link with multiple environmental and socio-economic impacts of food consumption in Switzerland. This link is established by food choices, which differ between population subgroups, and, as impact intensities differ between food groups, sociodemographic and lifestyle factors relate to impact levels. By disentangling these effects, entry points for policies become apparent. On the one hand, the established links between population subgroups and impact intensities can serve as entry point for policies aiming at reducing total impacts of a population. On the other hand, population subgroups that perform better than others can serve as example for favourable practices. With this, actions for improvements of the sustainability of diets and measures to hedge against potential negative side effects of these actions can ultimately be identified and targeted more effectively.

\section{Acknowledgements}

The contribution of AF, AM, and CS to this work was supported by the Swiss National Science Foundation (National Research Programme 69: "Sustainable and healthy diets: Trade-offs and synergies", project 4069- 166765). GP was supported by the Swiss Federal Food Safety and Veterinary Office (FSVO), project number 5.17.02ERN. We thank the FSVO for providing the menuCH dataset, and Carsten Nathani (Rütter Soceco AG) for providing Social Hotspots Index coefficients for input sectors. Further, we thank Bas Engel (Mathematical and Statistical Methods Group, Department of Plant Sciences, Wageningen University and Research, The Netherlands) for advice in setting up the statistical assessment. 
50 | 


\section{Chapter 4}

\section{Consumer strategies towards a more sustainable Swiss food system}

A. Frehner ${ }^{\mathrm{a}, \mathrm{b}}$, I.J.M. De Boer ${ }^{\mathrm{b}}$, A. Muller, ${ }^{\mathrm{a}, \mathrm{d}}$, H.H.E. Van Zantenc, C. Schader ${ }^{\mathrm{a}}$

aDepartment of Socioeconomics, Research Institute of Organic Agriculture FiBL, Frick, Switzerland

${ }^{\mathrm{b}}$ Animal Production Systems group, Wageningen University \& Research, Wageningen, the Netherlands

'Farming Systems Ecology group, Wageningen University \& Research, Wageningen, the Netherlands

dInstitute of Environmental Decisions, Federal Institutes of Technology Zurich ETHZ, Zurich, Switzerland

Submitted 


\section{2 | Chapter 4}

\section{Abstract}

To improve the sustainability performance of food systems, consumption- as well as productionside strategies are needed. We assessed multiple sustainability impacts of six consumer strategies for Switzerland. Two strategies encompassed dietary changes: following a vegetarian diet and adhering to the national dietary guidelines. Two strategies employed alternative farming systems: increasing the share of organic production and, in addition, applying the circularity principle of avoiding feed-food competition by limiting livestock feed to low-opportunity-cost biomass. Furthermore, in one strategy, food waste was reduced, and one strategy increased the share of domestic produce. Of these strategies, we assessed greenhouse gas emissions, land use, nitrogen surplus, social risks, diet quality, and diet costs. The different consumer strategies revealed tradeoffs between impact categories, unless combined in a synergistic way. While dietary changes towards more plant-based diets improved environmental impacts as well as diet quality, they increased social risks. Further, when the share of organic produce was increased, land use and dietary costs were increased. The effect on land use could however be reversed when circularity principles were introduced in addition to the organic production standard, resulting in improvements for all environmental indicators. Reducing food waste and increasing the share of domestic produce improved the sustainability performance as well, but at lower orders of magnitude. Combining all proposed strategies could lead to substantial improvements on all impact categories assessed, but would require a thorough transformation of the current food system. Yet, also the sum of individual consumers following one of the strategies proposed makes up an important contribution towards improving the sustainability performance of the Swiss food system. 


\section{Introduction}

In most high-income countries, current food consumption habits and the associated food production cause substantial impacts on multiple sustainability dimensions (Poore and Nemecek, 2018; Willett et al., 2019). Food consumption is an important determining factor for human health, and the underlying link between dietary patterns and non-communicable diseases, such as diabetes, cardiovascular disease, stroke, and cancer, is well established (Afshin et al., 2019). The transition towards diets containing more processed and refined products with a higher share of animal-source food (ASF) has substantially aggravated this. Further, current food production practices contribute substantially to approaching or already transgressing multiple planetary boundaries (Steffen et al., 2015). The latest report by the Intergovernmental Panel on Climate Change found that agriculture, including agriculturally-driven land use change, currently contributes around $23 \%$ to the total anthropogenic greenhouse gas (GHG) emissions (IPCC, 2019b). Moreover, agricultural production substantially altered and still alters the earth's biogeochemical cycles (Campbell et al., 2017). In addition to that, around $40 \%$ of the earth's land surface is currently used as croplands and pastures (Foley et al., 2011). Of these croplands, 40\% are used for feed production (Mottet et al., 2017), although using these croplands for the production of direct human food would be more efficient (Foley et al., 2011).

Strategies to improve the sustainability of our current food systems target consumption as well as production changes (Poore and Nemecek, 2018; Willett et al., 2019). On the consumption side, oftenproposed strategies focus on (partly) substituting ASF by plant-source food (Arrieta and González, 2018; Frehner et al., 2020; Poore and Nemecek, 2018). On the production side, strategies range from sustainable intensification (Pretty et al., 2018) to more extensive agricultural practices, such as organic (Muller et al., 2017). Further, a concept receiving increased attention is captured by 'circular food systems', which combines consumption- and production-related changes (De Boer and Van Ittersum, 2018). This concept is driven by the aim to allocate resources effectively within a food system, aiming at using resources for human food production first, and using only biomass unsuitable or unwanted by humans as animal feed. Consequently, the share of ASF in human food consumption decreases. Moreover, many initiatives propose an increase of local produce to enhance food sustainability (Moschitz and Oehen, 2020). Although some studies combine consumption- and production-side strategies in their assessments (Willett et al., 2019), consumption-side strategies were so far not assessed together with production-side aspects such as organic and circularity principles. Furthermore, while the majority of studies assessing sustainability aspects of more sustainable dietary choices did include environmental impacts and human health, social as well as economic aspects were rarely considered.

To this end, we aimed to assess multiple sustainability impacts of consumption- and productionside strategies towards more sustainable food systems. These strategies encompass dietary changes - reducing meat consumption and following dietary guidelines - as well as alternative farming and food system practices, such as organic production and circularity principles, and practices such as reducing food waste and increasing the share of domestic produce. Of these strategies, we assessed multiple sustainability impacts; three environmental indicators (GHG emissions, land use, and 


\section{4 | Chapter 4}

nitrogen surplus), social risks by the Social Hotspots Index (SHI) (Benoit-Norris et al., 2012), dietary costs, and diet quality by the Alternate Healthy Eating Index (AHEI) (Chiuve et al., 2012). In order to make results tangible, we present the assessed strategies at a consumer level. With this, we aim to initiate a discussion on the potential of including narratives into assessments of strategies towards more sustainable food systems. By applying this consumer strategy approach to Switzerland, we can draw conclusions with regard to effectiveness, synergies, and trade-offs of the different strategies. Moreover, the proposed approach of employing example consumer strategies is a promising way of engaging with stakeholders from different geographical, socio-economic, and cultural settings.

\section{Material and methods}

\section{I Consumer strategies}

We developed six consumer strategies, which include a range of common strategies towards more sustainable food systems. The development of these strategies was initiated and accompanied by several stakeholder workshops with policy makers as well as representatives of different institutes and population groups within the case study country Switzerland. In the initial workshop, ideas for possible strategies were inventorised. Based on this initial inventory, we developed six potential strategies for more sustainable food systems in Switzerland. In a next step, these strategies were translated into example consumer strategies, to improve their potential for communication with and adoption of different stakeholder groups (Appendix C1).

Although all strategies related to consumers, they included both changes on the consumption and changes on the production side. The strategies thus spanned from altering the food composition of diets to altering the amount of food wasted by consumers, and from altering the origin of consumed products to altering the production of foods. Table 1 summarises the main assumptions of the example consumer strategies. In order to compare all consumer strategies to a reference, we calculated a reference consumer strategy based on the most recent dietary recall dataset menuCH (Chatelan et al., 2017a; Frehner et al., 2021).

In strategy 1, meat of any kind in the human diet was reduced. We modelled this by employing three levels of meat reduction: $-25 \%,-50 \%$, and $-100 \%$. The example consumer of strategy 2 followed the Swiss nutritional guidelines (Swiss Food Pyramid SFP). Also here, we assessed different levels of implementation: $25 \%, 50 \%$, and $100 \%$, where this part of the diet was defined according to the $\mathrm{SFP}$, and the remaining part was defined according to the reference consumption. The consumer of strategy 3 reduced food waste at consumption stage, at the two levels $-25 \%$ and $-50 \%$.

In strategy 4, we assumed an increase of domestically produced food products to a minimum of $50 \%$ over all food groups that can be produced in Switzerland (Appendix C1). Where the current level of domestic production was currently already above $50 \%$, this higher level was kept constant. We only assumed changes in origin of final food products, and not of input products, such as feedstuffs.

Strategy 5 represents a consumer that increased the consumption of organically certified food products, which we modelled with three different levels (over all food groups): $25 \%, 50 \%$, and 100\% 
of organic produce in the human diet. To represent organic agriculture in our assessment, we excluded mineral fertiliser and assumed organic yields as identified by Seufert et al. (2012), see Stolze et al. (2019a) and Muller et al. (2020) for further information.

Moreover, in strategy 6, different alternative production practices were combined, by considering organic produce in combination with principles from circular agriculture. We implemented this likewise at three different levels of organic produce $(25 \%, 50 \%$, and $100 \%$ organic produce in the human diet), and furthermore applied the principle from circular agriculture that limits animal feed to low-opportunity-cost biomass (LCB) (De Boer and Van Ittersum, 2018). More concretely, according to this principle, animals are only fed with products that do not compete with human food production. These are products that are not used for human food consumption, such as byproducts, food waste, and grass resources. When applying this principle, animals can effectively upcycle LCB, and these resources can thereby be recycled into the food system (Van Hal et al., 2019a; Van Zanten et al., 2018). Since we employed consumer strategies at the level of individuals, we were able to assess this strategy without linking it to dietary changes. However, at food systems level, such a strategy would only be feasible in combination with reduced animal numbers and consequently reduced consumption of ASF.

Table I. Main assumption and implementation level of the example consumer strategies. $M=$ meat, $S F P=$ Swiss Food Pyramid, $\mathrm{FW}=$ food waste, $\mathrm{DOM}=$ domestic, $\mathrm{ORG}=$ organic, $\mathrm{STD}=$ standard feeding, $\mathrm{CIR}=$ circular agricultural principles.

\begin{tabular}{|c|c|c|}
\hline Strategy name and identifier & Description & Level \\
\hline Strategy 1: M25, M50, M100 & Reduction of meat consumption & $-25 \%,-50 \%,-100 \%$ \\
\hline Strategy 2: SFP25, SFP50, SFP100 & $\begin{array}{l}\text { Adherence to food-based dietary } \\
\text { guideline SFP }\end{array}$ & $25 \%, 50 \%, 100 \%$ \\
\hline Strategy 3: FW25, FW50 & Reduce food waste & $-25 \%,-50 \%$ \\
\hline Strategy 4: DOM50 & Increase domestic produce & Min. $50 \%$ \\
\hline $\begin{array}{l}\text { Strategy 5: ORG25_STD, } \\
\text { ORG50_STD, ORG100_STD }\end{array}$ & Increase share of organic produce & $25 \%, 50 \%, 100 \%$ \\
\hline $\begin{array}{l}\text { Strategy 6: ORG25_CIR, } \\
\text { ORG50_CIR, ORG100_CIR }\end{array}$ & $\begin{array}{l}\text { Increase share of organic produce in } \\
\text { combination with circular agriculture } \\
\text { principles }\end{array}$ & $25 \%, 50 \%, 100 \%$ \\
\hline
\end{tabular}

\subsection{Modelling approach and impact assessment}

We assessed multiple sustainability impacts of the consumer strategies using the biophysical massand nutrient-flow model SOLm (Muller et al., 2017; Schader et al., 2015). SOLm encompasses all mass- and nutrient-flows that are relevant for agricultural production. Of these, resource use and emissions are calculated, and by employing characterisation factors, these could be aggregated to GWP, land use, and nitrogen surplus (N surplus). Further, three additional indicators were calculated: the SHI on the production side, and the AHEI as well as costs of food consumption on the consumption side. 


\section{6 | Chapter 4}

\subsubsection{Environmental impact assessment}

We performed an environmental impact assessment of each level of the example consumer strategies using the biophysical mass-flow model SOLm (Muller et al., 2020; Muller et al., 2017; Schader et al., 2015). For the two stages in-between the farm-gate and the consumer, processing and transport, Ecoinvent 3 inventories were used (Appendix C2). The mass-flow model SOLm represents relevant flows of masses and nutrients that occur during agricultural production. Thereby, it allows to track resource use and emissions throughout the production processes, forming the basis for the environmental impact assessments GWP, land use, and N surplus. SOLm includes 192 countries, 180 primary crop and 22 primary farmed animal activities. These activities are characterised using FAOSTAT data for production and trade, as well as food balance sheets (FAO, 2001; FAOSTAT, 2018). Considering the focus on Switzerland in this study, we employed current production in Switzerland to define whether certain products can be produced in Switzerland or not, and further used the current countries of origin per food product (Frehner et al., 2021).

\subsubsection{Assessment of social risks}

Social risks were assessed based on the Social Hotspots Database (SHDB) (Benoit-Norris et al., 2012). This database covers 156 social indicators with risk levels per country and per sector in five areas: labour rights and decent work, health and safety, human rights, governance, and community infrastructure. For this study, mainly the sector agriculture is relevant, which is represented by 22 sub-sectors, of which 18 directly relate to food production. Social risks occur directly (in the respective food-related production sectors) and indirectly (in the sectors that produce upstream resources entering the food production, such as pesticide production). Indirect social risks were estimated using an input-output table for Switzerland (Nathani et al., 2011), in which the interlinkages between different industries as well as between industries and final demand of the economy are considered. By this, we capture social risks up to farm-gate. Using a weighting scheme, the social indicators were aggregated to the Social Hotspots Index (SHI) (Benoit-Norris et al., 2012; Norris et al., 2014).

\subsubsection{Diet quality assessment}

Although the final human health impact of different diets depends on a multitude of factors, indices can help to assess diet quality. Here, we employed the Alternate Healthy Eating Index (AHEI), which is a dietary index that was developed based on correlations of food groups and changes in human health performance (Chiuve et al., 2012). It correlates well with diseases such as coronary heart disease, diabetes, and the risk of stroke and cancer (Schwingshackl et al., 2018; Waijers et al., 2007). To calculate the AHEI, amounts of 11 food and nutrient categories are needed, and based on intake thresholds, a score from 1 to 10 per food category is assigned. In total, this thus leads to a maximum achievable score of 110 .

\subsubsection{Dietary cost assessment}

To complete an assessment of dietary costs that are associated to the consumer strategies, we collected price data of 94 commodities (Appendix C3). By this, we derived an estimation of the cost 
associated with the respective diet, which was driven by food group (e.g. meat vs. pulses) and production standard (e.g. organic vs. conventional). This indicator thus only captures differences in cost arising from the different diets, and not e.g. where these are consumed (in-house or gastronomy). For the purpose at hand, this was sufficient, as we only altered food groups and production standards in the consumer strategies. Moreover, since we were interested in the differences between consumer strategies, absolute values play a minor role. Assuming that relative prices are comparable between retailers, the use of only one retailer (Coop) can be justified. To derive the cost estimates per consumer strategy, the prices per commodity were multiplied by the quantities per commodity assumed in the respective strategy, which in total yielded the estimated dietary cost.

\section{Results}

\section{I Required behavioural changes of the consumer strategies}

Consumer strategies 1 and 2 require actual changes in the diet composition. Figure 1 shows the food compositions of these two strategies for their most extreme level (M100 and SFP100), as well as the food composition of the reference for comparison. The main change in the food composition of strategy 1 is characterised by a decrease of meat in the diet, which is replaced by pulses (Figure 1, middle). Notably, the remaining fraction of the food group meat, fish and seafood of this strategy consists of fish and seafood. The second strategy is defined by the dietary guidelines of Switzerland (Figure 1, right). The food composition of the SFP is characterised by a substantial increase in vegetables, fruits, and dairy, as compared to the reference. Moreover, sweets and alcoholic beverages are decreased, and meat, fish and seafood are partly replaced by pulses and dairy. The food composition of strategies 3-6 is represented by the reference composition (Figure 1, left).

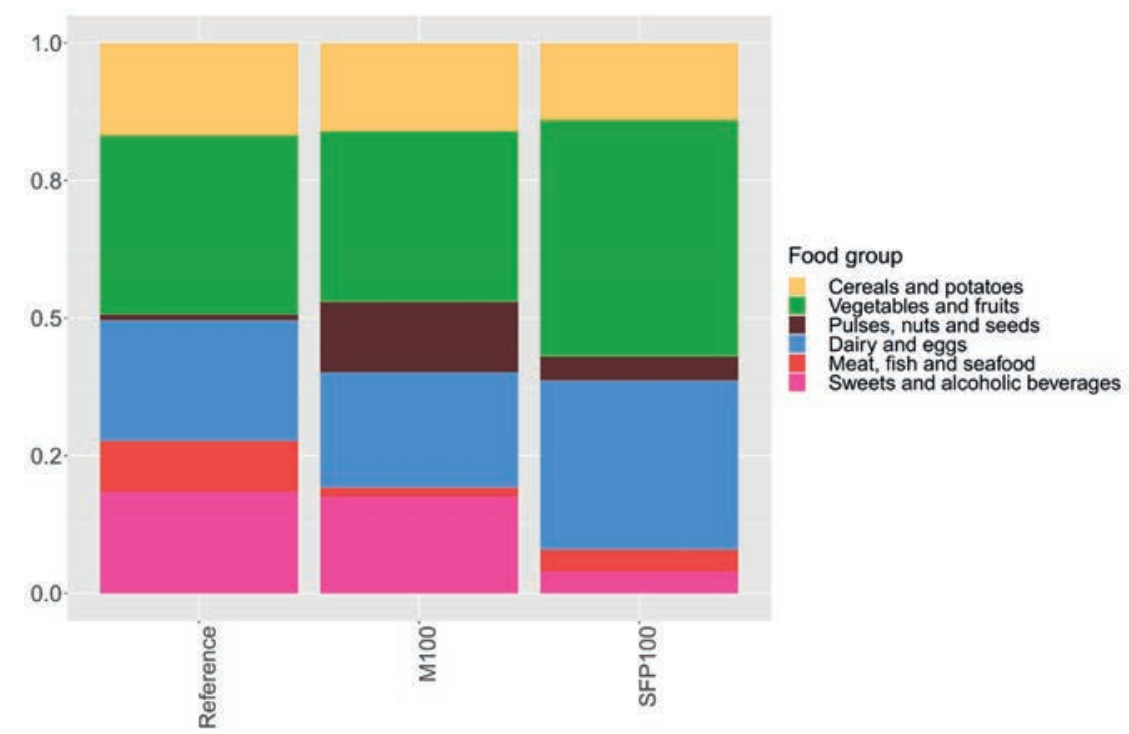

Figure I. Food composition of the consumer strategies. Share per food group shown in fresh matter. $M=$ meat, SFP $=$ Swiss Food Pyramid. 
For consumer strategies 3 to 6, food waste and purchasing behaviour are targeted. Thus, in strategy 3 , food waste at consumption stage needs to be reduced by $25 \%$ and $50 \%$ for the different levels. In strategies 4-6, purchasing behaviour needs to be adapted, with alternations in the origin of the products (strategy 4 ) and the production standard organic (strategies 5 and 6).

\subsection{Impacts of the consumer strategies}

The direction of the performance per consumer strategy, level, and impact category is shown in Figure 2, relative to the reference consumer. Strategies 1 and 2 revealed a similar pattern, thus improving all impact categories, except a reduced performance regarding social risks (i.e. increased social risks). In both strategies 1 and 2, this was largely driven by a replacement of meat products with plant-sourced foods, such as legumes and vegetables. Notably, when the strategy 1 was implemented by $25 \%$ only, all impact categories improved, but to a lesser extent than with a stricter implementation (50\% and 100\%). With stricter implementation, however, social risks increased compared to the reference. Strategy 3 was the only strategy that had exclusively positive effects (except for no effect on the nutritional quality index). Further, strategies 4 and 5 improved $N$ surplus and partly the SHI. They however also increased land use, and strategy 5 moreover partly increased GWP as well as diet cost. Finally, strategy 6 improved all environmental impact categories, and did not affect the SHI and AHEI. However, as strategy 5, it increased dietary cost.

The different strategies revealed both synergies and trade-offs between the different impact categories (Figure 3). Notably, N surplus was the only impact category with unambiguous signals, meaning that all strategies led to a lower $\mathrm{N}$ surplus than the reference consumer strategy. For the other environmental indicators, land use - and partly GWP - revealed trade-offs for strategies 5 and 6 , which resulted from the organic production standard employed. In fact, since organic production comes along with lower yields, the land used to reach the same amount of produce increases. As shown in Figure 2, increases in land use of strategy 5 went up 33.3\%, while increases in GWP were minor (up to $1 \%$ increase). Remarkably, these trade-offs diminished when circular food system principles were employed in addition to organic production standards: in this case, all environmental impacts showed improved performance. Hence, the environmental impacts of organic produce was highly dependent on whether this production standard was accompanied by additional measures regarding animal feeding regimes (strategy 6), or not (strategy 5). 


\begin{tabular}{|c|c|c|c|c|c|c|c|}
\hline \multirow{2}{*}{$\begin{array}{l}\text { Consumer } \\
\text { strategy }\end{array}$} & \multirow[t]{2}{*}{ Level } & \multicolumn{6}{|c|}{ Impactcategory } \\
\hline & & $\sum_{0}^{0}$ & 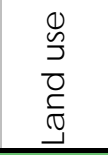 & $\begin{array}{l}\frac{n}{2} \\
\frac{2}{2} \\
\bar{n} \\
z\end{array}$ & 焉 & 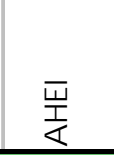 & $\begin{array}{l}\text { th } \\
0 \\
ن\end{array}$ \\
\hline \multirow[t]{3}{*}{ Strategy 1} & M25 & $-14.8 \%$ & $-17.1 \%$ & $-14.9 \%$ & $-1.8 \%$ & $2.1 \%$ & $-6.0 \%$ \\
\hline & M50 & $-29.1 \%$ & $-32.5 \%$ & $-28.7 \%$ & $0.0 \%$ & $13.6 \%$ & $-11.0 \%$ \\
\hline & M 100 & $-55.3 \%$ & $-56.0 \%$ & $-51.0 \%$ & $18.9 \%$ & $17.9 \%$ & $-17.0 \%$ \\
\hline \multirow[t]{3}{*}{ Strategy 2} & SFP25 & $-8.5 \%$ & $-8.3 \%$ & $-8.0 \%$ & $2.6 \%$ & $14.3 \%$ & $-6.9 \%$ \\
\hline & SFP50 & $-17.0 \%$ & $-16.1 \%$ & $-16.6 \%$ & $5.3 \%$ & $28.6 \%$ & $-13.8 \%$ \\
\hline & SFP100 & $-34.0 \%$ & $-33.2 \%$ & $-32.2 \%$ & $10.6 \%$ & $57.3 \%$ & $-27.6 \%$ \\
\hline \multirow[t]{2}{*}{ Strategy 3} & FW25 & $-1.9 \%$ & $-1.9 \%$ & $-2.0 \%$ & $-2.8 \%$ & $0.0 \%$ & $-2.4 \%$ \\
\hline & FW50 & $-3.8 \%$ & $-3.7 \%$ & $-4.0 \%$ & $-5.6 \%$ & $0.0 \%$ & $-4.9 \%$ \\
\hline Strategy 4 & DOM 50 & $-0.3 \%$ & $2.0 \%$ & $-0.4 \%$ & $-20.2 \%$ & $0.0 \%$ & $0.0 \%$ \\
\hline \multirow[t]{3}{*}{ Strategy 5} & ORG25_STD & $0.3 \%$ & $8.3 \%$ & $-15.0 \%$ & $0.0 \%$ & $0.0 \%$ & $10.6 \%$ \\
\hline & ORG50_STD & $0.5 \%$ & $16.7 \%$ & $-30.0 \%$ & $0.0 \%$ & $0.0 \%$ & $21.1 \%$ \\
\hline & ORG 100_STD & $1.0 \%$ & $33.3 \%$ & $-60.0 \%$ & $0.0 \%$ & $0.0 \%$ & $42.2 \%$ \\
\hline \multirow[t]{7}{*}{ Strategy 6} & ORG25_CIR & $-7.3 \%$ & $-12.5 \%$ & $-18.8 \%$ & $0.0 \%$ & $0.0 \%$ & $10.6 \%$ \\
\hline & ORG50_CIR & $-14.6 \%$ & $-28.1 \%$ & $-37.5 \%$ & $0.0 \%$ & $0.0 \%$ & $21.1 \%$ \\
\hline & ORG100_CIR & $-29.3 \%$ & $-68.9 \%$ & $-75.0 \%$ & $0.0 \%$ & $0.0 \%$ & $42.2 \%$ \\
\hline & & \multicolumn{2}{|c|}{ Improvement } & Detriment & & \multicolumn{2}{|c|}{ No difference } \\
\hline & & & \multicolumn{2}{|l|}{$<20 \%$} & \multicolumn{3}{|l|}{$<20 \%$} \\
\hline & & & \multicolumn{2}{|l|}{$20-40 \%$} & \multicolumn{3}{|l|}{$20-40 \%$} \\
\hline & & & \multicolumn{2}{|l|}{$>40 \%$} & \multicolumn{3}{|l|}{$>40 \%$} \\
\hline
\end{tabular}

Figure 2. Option space per strategy and level: change in performance is indicated per impact category (improved performance, detriment performance, no difference). All changes in performance are relative to the reference consumer strategy. With the exception of the AHEI, improvement relates to a decrease, and detriment to an increase. $M=$ meat, SFP = Swiss Food Pyramid, FW = food waste, $\mathrm{DOM}=$ domestic, $\mathrm{ORG}=$ organic, $\mathrm{STD}=$ standard feeding, $\mathrm{CIR}=$ circular agricultural principles, GWP = global warming potential, $\mathrm{N}=$ nitrogen, $\mathrm{SHI}=$ Social Hotspots Index, $\mathrm{AHEI}=$ Alternate Healthy Eating Index.

For the social risks (SHI), strategies 1 and 2 showed clear trade-offs; the increase in plant-based products, such as legumes (strategy 1 and 2) as well as vegetables and fruits (strategy 2), increased social risks by up to $18.9 \%$ (strategy 1 ) and $10.6 \%$ (strategy 2 ). This effect was mainly driven by imports: typical importing countries of these plant-based products showed higher occurrence of social risks, which triggered this increase. Thus, while all other impact categories performed better than in the reference consumer strategy, social risks increased for these strategies. For strategy 3 and 4 , social risks decreased, which was driven by a total reduction in consumed products (strategy 3 ) and lowered imports (strategy 4 ). In strategy 4 , the substantial decrease (-20.2\%) could be explained by lower social risk pressures in Switzerland, as opposed to the countries of origin of the reference consumer strategy. For strategies 5 and 6, it has to be noted that differences in social standards between organic and conventional production were not considered in the SHI. Therefore, potential differences in social risks could not be captured by the measure employed, and results thus suggested that no difference in social risks occurred. 
क्ष

$\frac{\bar{\Phi}}{\stackrel{\Phi}{8}}$

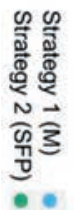

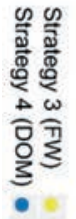

恋

융ํํ

คำ

递学

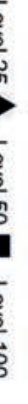

\begin{abstract}
(
\end{abstract}

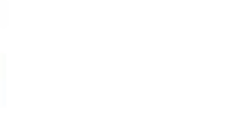

列
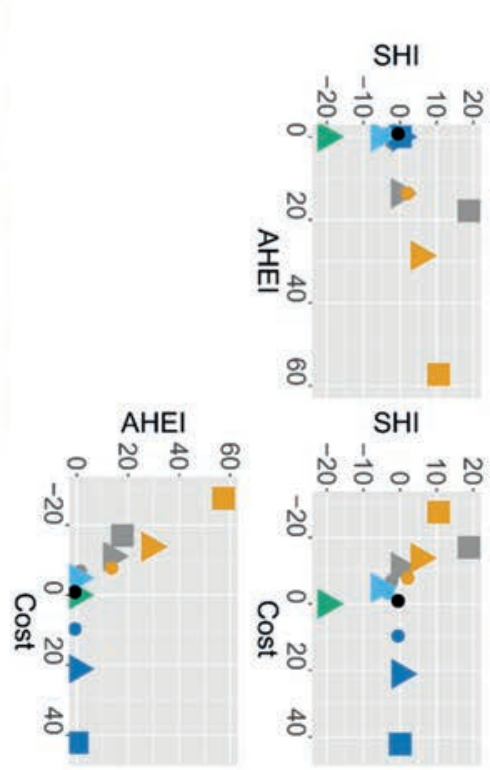

กิ.

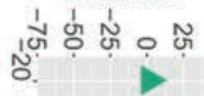

立.

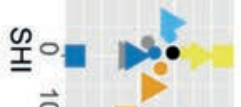

$\overrightarrow{0}$.
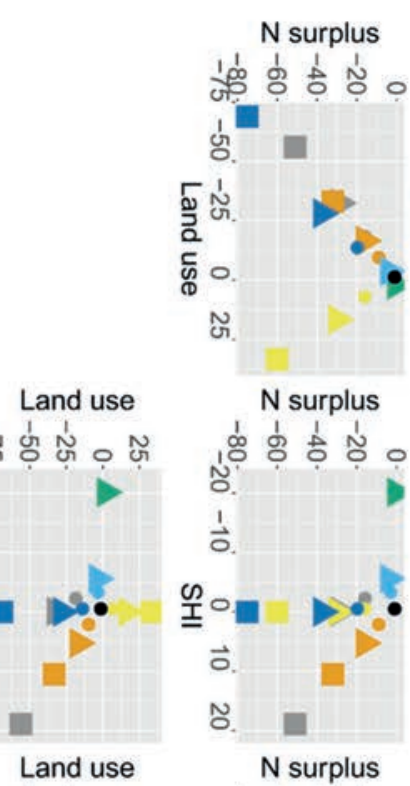

GWP

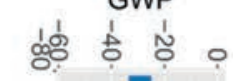

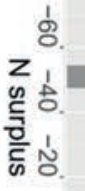

111

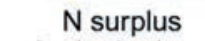

సึ.

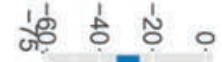
잉

送

‥

$\stackrel{1}{\circ}$
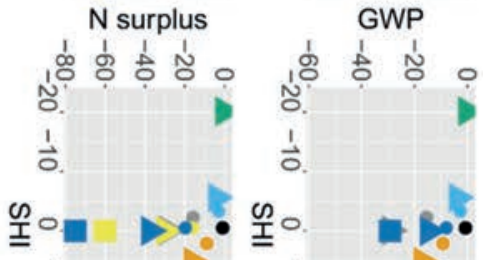

$\overrightarrow{0}$

ก.

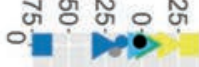

surplus

옹.

o.

กั.

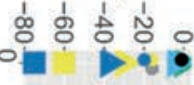

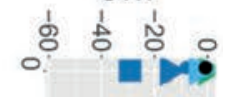

ㄷ. $1>0$

盍。

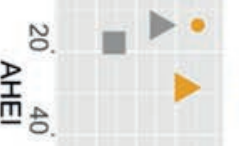

8.

8.
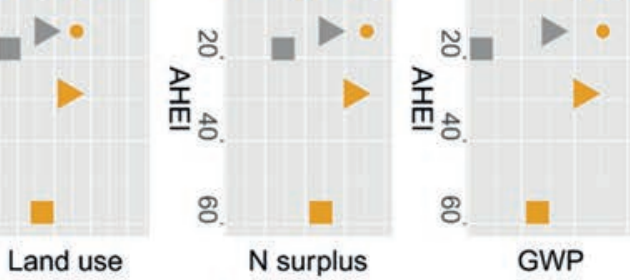

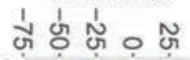
กิ.

ํํㅇํํ우 웅
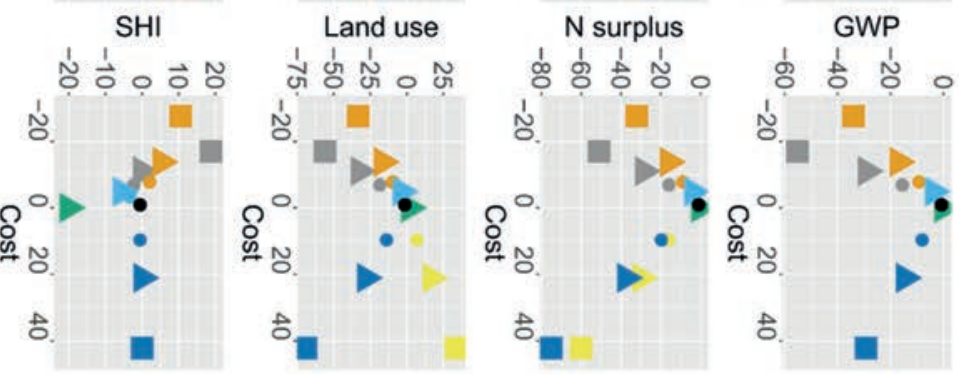

Figure 3. Combinations of deviations of all impact categories on the $x$ - and $y$-axis. The symbols represent the change in the respective impact category of the strategies relative to the reference (black dot). $M=$ meat, SFP = Swiss Food Pyramid, FW = food waste, $\mathrm{DOM}=$ domestic, ORG = organic, STD = standard feeding, CIR = circular agricultural principles. 
The AHEI was, next to N surplus, the only impact category where no strategy led to a reduction. For strategies 1 and 2, the AHEI increased (up to $+17.9 \%$ for strategy 1 , and up to $+57.3 \%$ for strategy 2 ). Especially the increase for strategy 2 was substantial, which was mainly driven by a decrease in red and processed meat, an increase in nuts and legumes, whole grains, and nuts and vegetables. These two strategies were the only ones with changed food composition, and the AHEI of strategies 3-6 was consequently not affected.

Cost of the different strategies was driven by two factors: changes in food composition, and changes in production standards (organically labelled products with a price premium, see Appendix C3). Hence, dietary cost was reduced (its performance thus improved) for strategies 1-3 - in strategy 3, this was moreover driven by the total reduction in consumed foods. On the contrary, dietary cost was increased in strategies 5 and 6 . Hence, also this impact category led to trade-offs for strategies 5 and 6; while most environmental impacts were improved, partly even substantially, dietary cost was increased due to higher costs associated with organic produce.

In summary, all consumer strategies that revealed the highest reduction potential (strategy 1 for GWP: $-55.3 \%$, strategy 6 for land use; $-68.9 \%$, strategy 6 for $\mathrm{N}$ surplus: $-75 \%$, strategy 4 for social risks; $-20.2 \%$, strategy 2 for the AHEI: $+57.3 \%$, strategy 2 for cost: $-27.6 \%$ ) revealed trade-offs with other impact categories. These trade-offs occurred either with social risks (strategies 1 and 2), land use (strategy 4), or dietary cost (strategies 5 and 6). Strategy 3 was unique in the sense that it only revealed synergies; these were however of lower orders of magnitude than for the other strategies (SHI: reduction up to $5.6 \%$, cost: reduction up to $4.9 \%$, GWP, N surplus, and land use: reductions up to about $4 \%$ each).

\section{Discussion}

Our results showed that large improvement potentials on one or more impact categories come along with trade-offs, thus detrimental performance on other impact categories. Only food waste reduction improved - or at least kept constant - all impact categories, but improvement potentials were of lower orders of magnitude than for the other strategies. Consequently, we found that clear priorities are needed to achieve substantial improvements. For the assessment of environmental impacts of the Swiss Food Pyramid, our results are in line with previous assessments carried out by Chen et al. (2019). These authors found an average reduction of $36 \%$ across five different environmental impact categories for the Swiss Food Pyramid, while we found reductions between 32 and $34 \%$ for the different environmental impact categories assessed. Moreover, for cost of diets, Chen et al. (2019) found a reduction of 35\% for the Swiss Food Pyramid, while our estimates suggest a reduction of $28 \%$, thus also in the same order of magnitude. To our knowledge, no direct comparison is available for the other impact categories and strategies for the Swiss case study.

\section{I Combination of strategies}

By a smart mix of strategies, it is possible to improve all impact categories assessed simultaneously, thus create synergies. More concretely, by a combination of better performing consumption patterns (strategies 1 and 2; less animal-source foods, healthier plant-based foods), reductions of food waste 
(strategy 3), and changes in production (strategies 5 and 6; e.g. organic standards in combination with circular food system principles). By this combination, less land would be needed to produce the required food items, which would in turn allow to grow more foods domestically (strategy 4). In this combined approach, many trade-offs could be solved - e.g. the increase of social risks in strategies 1 and 2 could be reduced by being able to grow more domestically (strategy 4). Moreover, by such combinations, inconsistencies in single strategies - for example in strategy 1 , where meat is excluded completely while dairy is still consumed, whereby the meat resulting from dairy production is not consumed domestically anymore - could be solved. While such a combination of strategies would require a thorough transformation of the current food system, smaller changes can be achieved by different consumers following different profiles. In fact, when different consumers follow one of the strategies proposed, the sum of their actions tackling different strategies can also result in an - although less strong - effect. Yet, following single strategies can have the advantage of being both easier to communicate and to implement.

\subsection{Narratives based on consumer strategies}

Narratives constitute a communication format that relies on cause-and-effect relationships between events and particular characters (Dahlstrom, 2014). While narratives are generally rarely considered in scientific discourse, they could lead to a larger reach-out and more effective communication, since narratives dominate much of the public attention (Green and Brock, 2000). Recently, narratives have for example been employed, and shown to be effective, for the communication of healthier dietary choices (Niederdeppe et al., 2019; Shimazaki et al., 2018). Moreover, Yang and Hobbs (2020) have demonstrated the potential of employing narratives in science communication, with substantially better information uptake of stakeholders when confronted with narratives, versus logical-scientific information framing. With the consumer strategies approach proposed here, we provide a scientific basis for employing narratives in the communication of more sustainable dietary choices. By enhancing the consumer strategies with contexts and motivations for their respective behavioural change, a more consumer-oriented communication approach can be accomplished. Employing narratives in science communication in the transition towards more sustainable food systems could thus offer potential for improving information uptake, but further research is needed in designing as well as testing the effectiveness of such approaches.

\subsection{Policy implications}

Policy implications differ between the (combinations of) strategies. On the one hand, policies targeting at changing consumers' behaviours could do so by measures such as information campaigns, enhanced nudging, or financial mechanisms to influence relative prices (Latka et al., 2021). Alternatively, measures such as bans could be introduced, which however impair each person's freedom substantially. On the other hand, given the importance that individuals are targeted in interaction with the wider systemic environment (Hoek et al., 2021), policies targeting at changed production can include information and education, financial instruments, and obligations. Further, in order to increase transparency about changes in production for consumers, labels, such as the example of organic employed here, can play an important role (McFadden and Huffman, 
2017). Policies targeting at actions in between production and consumption could moreover steer allocation of resources. As an example, larger retailers not only dispose of buying power, but also influence consumers' behaviour via advertisements (Hawkes, 2008). Hence, advertisements for less sustainable products could thus be taxed higher, or even banned. Finally, when these policies are aligned, above-mentioned synergies can be exploited. This would require a coordinated action plan involving all relevant stakeholders, from production, processing, retail, gastronomy, transport, and consumers.

\subsection{Limitations}

Our approach comes with several limitations. First, results for the organic production standard need to be interpreted with care. We modelled organic in the food systems model SOLm, which was developed to capture the essentials of this production standard. In SOLm, organic is mainly characterised by lower yields according to Seufert et al. (2012), no mineral fertilisers and consequently reduced nitrogen inputs, and prohibition of non-organic pesticide use. In our assessment, however, potential advantages of organic agriculture, such as impacts on biodiversity, impacts of reduced pesticide use, and impacts on soil health (Meier et al., 2015; Van Der Werf et al., 2020), could not be captured. Furthermore, some organic regulations also include specific social standards (Seufert et al., 2017). In our assessment of social risks with the SHI, however, differences in production standards were not accounted for, and thus potential improvements of social risks of organic production was not captured.

Second, the indicators chosen to represent different sustainability dimensions need to be interpreted with care, since each of the dimensions is more complex than the single impact categories employed here. For example, the environmental dimension encompasses much more than land use, GHG emissions, and nitrogen surplus. Also, consequences of dietary change for human health are highly complex and depend on many factors (Conlon and Bird, 2015; Feigin et al., 2016). The AHEI indicator employed here can therefore only give a trend of a potential improvement or detriment, and not give exact estimates of these consequences (Chiuve et al., 2012). Moreover, with changed consumption and production patterns, costs per food item are likely to be influenced, resulting in changed prices on short-term - and vice versa, changes in prices influencing food demand (Andreyeva et al., 2010). Also this indicator needs, therefore, to be interpreted with caution, and solely gives an indication of potential effects.

\section{Conclusion}

The six consumer strategies revealed trade-offs between impact categories, unless combined in a synergistic way. While dietary changes towards more plant-based foods improved environmental impacts as well as diet quality, they could increase social risks. Further, when increasing the share of organic produce, land use and dietary costs were increased. The effect on land use could however be reversed when circularity principles were introduced in addition to the organic production standard, resulting in substantial improvements for all environmental indicators. 


\section{4 | Chapter 4}

Our results have implications for consumers, policy makers, as well as other food system actors. On the one hand, we showed that consumers following individual strategies can make important contributions towards more sustainable food systems. Moreover, there is potential in joining scientific results with narratives to user-tailored communication approaches, with increased potential for information uptake and implementation. On the other hand, a coordinated action plan that targets a thorough redesign of the food system and combines several of the proposed strategies is needed in order to reach large systemic effects. This could encompass suitable education measures, incentives, as well as rules for production, processing, retail, gastronomy, transport, and consumption.

\section{Acknowledgements}

We thank Carsten Nathani (Rütter Soceco AG) for providing Social Hotspots Index coefficients for input sectors, and Angelika Hayer (Swiss Society for Nutrition SSN) for inputs regarding interpretation of the Swiss dietary guidelines. The consumer strategies can be accessed at SNF (2020).The contribution of AF, AM, and CS to this work was supported by the Swiss National Science Foundation (National Research Project 69: "Sustainable and healthy diets: Trade-offs and synergies", project 4069- 166765). 


\title{
Chapter 5
}

\section{Integrating circularity principles with animal-source food recommendations in national dietary guidelines: nutritional and environmental consequences}

\author{
A. Frehner ${ }^{\mathrm{a}, \mathrm{b}}$, R.P.M. Cardinaals ${ }^{\mathrm{c}}$, I.J.M. De Boer ${ }^{\mathrm{b}}$, A. Muller ${ }^{\mathrm{a}, \mathrm{d}}$, C. Schader ${ }^{\mathrm{a}}$, B. Van Selm ${ }^{\mathrm{b}}$, O. Van Hal ${ }^{\mathrm{b}}$, \\ G. Pestonie,f, S. Rohrmanne, M. Herrerog, H.H.E. Van Zanten ${ }^{c}$
}

aDepartment of Socioeconomics, Research Institute of Organic Agriculture FiBL, Frick, Switzerland

${ }^{\mathrm{b} A n i m a l}$ Production Systems group, Wageningen University \& Research, Wageningen, the Netherlands

cFarming Systems Ecology group, Wageningen University \& Research, Wageningen, the Netherlands

dInstitute of Environmental Decisions, Federal Institutes of Technology Zurich ETHZ, Zurich, Switzerland

eDivision of Chronic Disease Epidemiology, Epidemiology, Biostatistics and Prevention Institute, University of Zurich, Zurich, Switzerland

${ }^{\mathrm{f} N u t r i t i o n}$ Group, Health Department, Swiss Distance University of Applied Sciences, Regensdorf, Switzerland gCommonwealth Scientific and Industrial Research Organisation, Australia

Submitted 


\section{6 | Chapter 5}

\section{Abstract}

Background. National food-based dietary guidelines are a common starting point for dietary improvements that reflect both cultural and geographical circumstances. These guidelines are generally designed from a nutrient requirement and diet quality perspective. Hence, they mostly disregard sustainability principles like resource suitability and environmental consequences of the associated food production. Our aim was to assess and integrate nutritional and environmental consequences of limiting national animal-source food (ASF) recommendations to livestock raised on circular production systems using low-opportunity-cost biomass. These circular food production systems will be required to achieve healthy diets from sustainable food systems.

Methods. We investigated whether ASF recommendations of five case study countries in Europe (Bulgaria, Malta, the Netherlands, Sweden, and Switzerland) could be met with nationally available low-opportunity-cost biomass only. We used an optimisation model where feed resources were allocated to different species of farm animals in order to optimise different nutritional outcomes for humans. Following this, we assessed global warming potential, land use, and the nutritional contribution of the resulting ASF quantities in national dietary guidelines.

Findings. The substantial ASF quantities recommended in the assessed food-based dietary guidelines prevented compatibility with the quantities and nutritional contribution of ASF from circular production systems. Maximum reachable protein contribution from low-opportunity-cost biomass ranged from $15.9 \mathrm{~g}$ protein per capita per day (Malta) to $38.9 \mathrm{~g}$ protein per capita per day (Switzerland). Although these protein contributions could cover almost one third up to half of daily human protein needs, they are substantially lower than the protein contributions of the ASF recommendations of the five national food-based dietary guidelines.

Interpretation. Limiting feeding of livestock to nationally available low-opportunity-cost biomass results in a more circular and climate-friendly food system. To reach this, ASF recommendations would need to be revised and lowered. This is consistent with recent efforts to include sustainability criteria in dietary guidelines. 


\section{Introduction}

The production of human food affects the environment in multiple ways, including its associated resource use that alters the global biomass and nutrient cycles, its climate change impacts, biodiversity loss, and others (Poore and Nemecek, 2018). Suboptimal diets are a major risk factor for several non-communicable diseases, such as cardiovascular diseases, stroke, cancer, and diabetes (Afshin et al., 2019). In high-income countries, shifting consumption towards plant-based diets is often recommended, both to decrease environmental impacts of food consumption and to improve the human health performance of diets. This is due to the generally favourable environmental performance of plant-based food in comparison to animal-source food (ASF) (Poore and Nemecek, 2018), as well as concerns of low consumption of fruits and vegetables, and high red and processed meat intake for human health (Afshin et al., 2019). Food-based dietary guidelines (FBDG) are key references for healthier food choices. Although environmental concerns are increasingly taken up in FBDGs, for example in the recently proposed EAT-Lancet guidelines (Willett et al., 2019) as well as in several national FBDGs, most national FBDGs still do not include sustainability criteria (Springmann et al., 2020). In comparison to globally applicable guidelines such as the EAT-Lancet, national FBDGs take geographical and cultural circumstances into consideration (Fischer and Garnett, 2016), and are hence often well embedded in education and nutrition counselling at national level (Brown et al., 2011).

While the necessity to reduce ASF is generally acknowledged, different solutions exist regarding how the remaining ASF could be produced, and which ASF should be reduced to what extent (Frehner et al., 2020). Studies operating with current environmental impact intensities often recommend to reduce ASF to a minimum, or to reduce beef and pork more than chicken. In these studies, land suitability is mostly not captured, neglecting that much of the land currently used to produce feed for animals could instead be used to grow plants for direct human consumption, resulting in feed-food competition (Mottet et al., 2017; Schader et al., 2015). Other studies that consider systemic consequences throughout the food system often propose to feed livestock on lowopportunity-cost biomass (LCB), i.e. grass resources, by-products from processing, and food waste only, and mostly recommend to reduce chicken and pork most (Frehner et al., 2020). The latter approaches thus include resource suitability and interlinkages between different food chains in their assessments, which constitute key principles of circular food systems (De Boer and Van Ittersum, 2018; Van Zanten et al., 2019). In circular food systems, resources are prioritised for human food first, and are allocated as animal feed with second priority (Muscat et al., 2019). Thereby, feed-food competition is largely avoided and biomass is consequently used more effectively. By considering this principle, animals can contribute to recycling biomass and nutrients back into the food system, which would otherwise be lost for human food consumption (Van Kernebeek et al., 2016). In sum, from an environmental and resource-use efficiency perspective, the role of ASF in FBDGs is thus contested.

Against this background, we investigated whether ASF recommended in national FBDGs can be produced without feed-food competition, and assessed the nutritional and environmental consequences. We explored the nutritional option space that ASF based on nationally-available LCB 
could provide, and what role ASF could play in balanced diets. Using a resource allocation model developed by Van Hal et al. (2019a), we assessed different scenarios for five European case study countries. Thereby, feed resources were allocated to different farm animals in order to optimise different nutritional outcomes for humans.

\section{Material and methods}

\section{I Study design and system boundaries}

Europe was selected as case study region due to its stable food security situation and high quality data, where discussions on food choices mainly revolve around issues regarding overconsumption and the search for healthy and sustainable food choices (WHO, 2018). Within Europe, dietary habits differ due to cultural habits and resource endowments. We identified all European countries that provide detailed dietary guidelines and selected five of them representing different regions with differing dietary habits as case studies: Bulgaria (East), Malta (South), the Netherlands (West), Sweden (North), and Switzerland (Central) (Appendix D1).

\subsection{Plant-based food recommendations}

After collecting the recommended amounts per food group of the selected FBDGs, we transformed the FBDG recommendations into a daily diet (per capita per day). For the disaggregation of food groups (e.g. cereals) into food items (e.g. wheat and products, rye and products), FAO Food Balance Sheets were employed (FAO, 2001) (Appendix D1). After these transformations, we obtained an example FBDG diet per country, of which we retained only the plant-based food part for the subsequent analysis. The availability of LCB (processing by-products, food waste, and grass resources) in each case study country was based on the recommended plant-based food intake when assuming the whole population would follow the country-specific FBDG diet (Appendix D2).

\subsection{Animal-source food scenarios}

We investigated the potential contribution of ASF to a balanced diet in four different scenarios for each FBDG. We thereby solely focussed on ASF and thus did not aim to provide realistic alternatives for the FBDGs employed, but rather to explore the option space of ASF based on LCB for different nutritional foci. The scenarios all met the circular food system principle of avoiding feed-food competition, meaning that ASF only originated from animals fed on LCB. Thus, only LCB that was produced within the country and resulted from the consumption of the plant-based food part of the respective national FBDGs was available as feed.

In the first alternative scenario, MaxProt, LCB was allocated to the different animal production systems such that human-digestible animal protein was maximised. Next to protein, ASF contains multiple essential nutrients for humans, such as for example essential fatty acids, vitamins $A, D_{3}$, and $B_{12}$, calcium, iron, and zinc (Adesogan et al., 2020). Often, it is however not clearly stated which nutrients are the reason to recommend different types of ASF in FBDGs, except for the macronutrients carbohydrates, fats, and proteins. To take the specific nutritional functions of ASF in 
the diet into account, we employed three scenarios that put different emphasis on three main nutrient groups: omega-3 fatty acids (Scenario MaxFattyAcids), minerals (Scenario MaxMinerals), and vitamins (Scenario MaxVitamins). In each of these scenarios, one of the respective groups of nutrients was maximised, instead of protein. The scenario MaxFattyAcids maximised the sum of the omega-3 fatty acids $\alpha$-linolenic acid (ALA), docosahexaenoic acid (DHA), and eicosapentaenoic acid (EPA) (in g), the scenario MaxMinerals the sum of the minerals calcium, iron, and zinc (in mg), and the scenario MaxVitamins the sum of the vitamins A and $B_{12}$ (in $\mu \mathrm{g}$ ). For each of the nutrients of a group to be maximised, a lower bound was set to the level achieved in the scenario MaxProt, in order to avoid disbalancing one nutrient by another. Applying these three scenarios can show which nutritional functions of the original FBDGs can be met with ASF from LCB, and which ASF products are essential for which nutritional functions.

\subsection{Resource allocation model}

We used a resource allocation model published by Van Hal et al. (2019a) to estimate potential ASF based on the calculated LCB (Van Hal, 2020). The model contains a detailed representation of seven animal production systems (dairy, beef, layer, chicken, pig, salmon, and tilapia), and allocates feed resources to the different animal production systems while maximising different nutritional contributions (Appendix D3).

\subsection{Nutritional contribution}

We quantified the following nutrient contributions of the FBDG diets: protein, calcium, iron, zinc, vitamin A, vitamin B12, and the omega-3 fatty acids ALA, EPA, and DHA (Appendix D1). Nutritional contributions were calculated with food composition tables (NEVO-table, 2019). By multiplying quantities per food item of the different FBDG diets as well as the scenarios with the nutrient contents, we derived the total nutritional contribution.

\subsection{Environmental impact assessment}

We assessed the global warming potential (GWP) and land use of the different scenarios using the biophysical mass-flow model SOLm (Muller et al., 2017; Schader et al., 2015). SOLm represents the relevant mass and nutrient flows of agricultural production, allowing to assess the consequences of large-scale changes in the food system on both resource use and emissions (Appendix D4).

\section{Results}

The compositions of the FBDGs of the case study countries differed by agro-ecological and sociocultural context (Figure 1). The protein contribution of the recommended foods of the countries investigated ranged from $60 \mathrm{~g}$ (Malta) to $98 \mathrm{~g}$ (Netherlands) per capita per day (Table 1). With this, all countries issued recommendations with higher protein contribution than the average daily protein requirements ( 50-60 g) (EFSA, 2017; WHO, 2007), whereby also protein requirements of population groups with higher requirements can be met (e.g. during pregnancy, or for elderly). 
Moreover, the share of ASF protein from total protein was remarkably high in all FBDGs assessed, ranging from 0.46 (Netherlands) to 0.66 (Sweden).

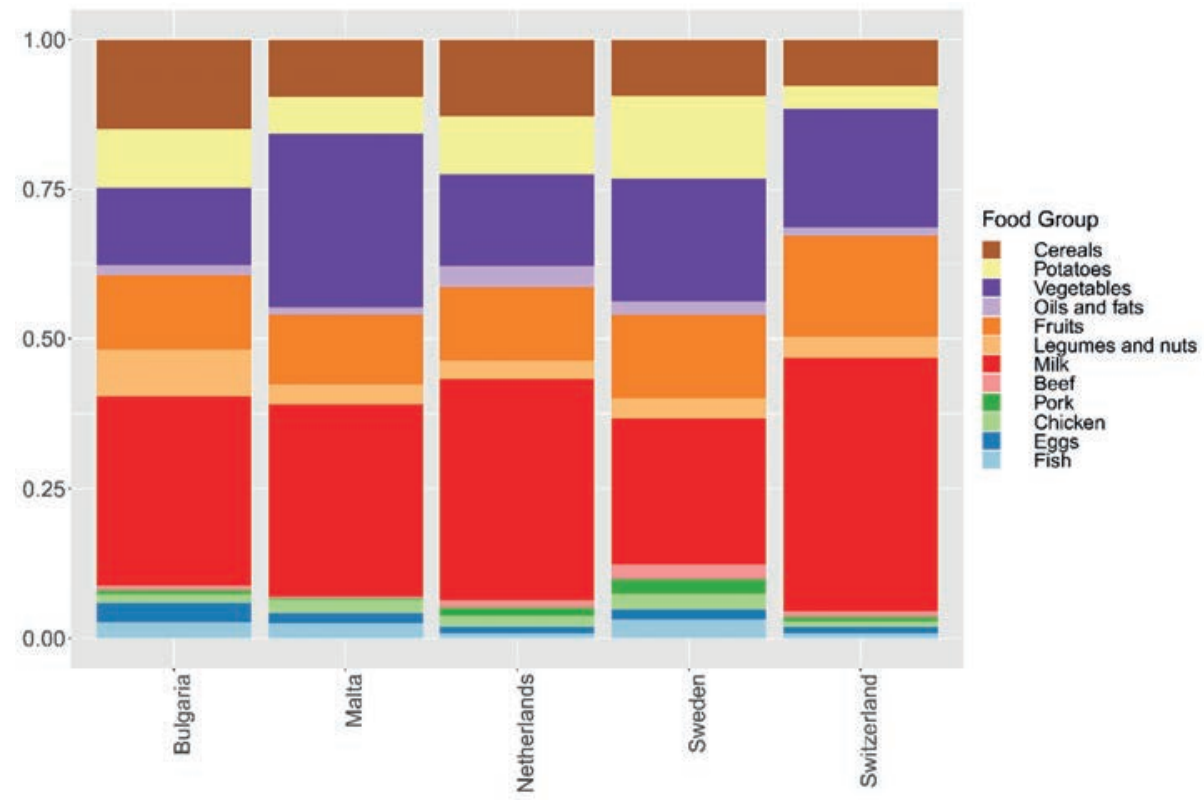

Figure I. Composition of the original FBDGs (in primary product equivalents, \% of total fresh weight).

Table I. Protein contribution of food-based dietary guidelines in five European countries. ASF: animal-source food; FBDG: foodbased dietary guidelines.

\begin{tabular}{llll}
\hline Country & $\begin{array}{l}\text { Total protein } \\
\text { contribution (FBDG) }\end{array}$ & $\begin{array}{l}\text { ASF protein } \\
\text { contribution (FBDG) }\end{array}$ & $\begin{array}{l}\text { Share ASF protein } \\
\text { / total protein FBDG }\end{array}$ \\
\hline Bulgaria & $93 \mathrm{~g}$ & $44 \mathrm{~g}$ & 0.47 \\
Malta & $60 \mathrm{~g}$ & $34 \mathrm{~g}$ & 0.56 \\
Netherlands & $98 \mathrm{~g}$ & $45 \mathrm{~g}$ & 0.46 \\
Sweden & $85 \mathrm{~g}$ & $56 \mathrm{~g}$ & 0.66 \\
Switzerland & $83 \mathrm{~g}$ & $44 \mathrm{~g}$ & 0.52 \\
\hline
\end{tabular}

The scenarios MaxProt, MaxFattyAcids, MaxMinerals, and MaxVitamins illustrate the option space of different nutritional foci (Figure 2). By the optimisation process employed, results were driven by relative efficiencies (i.e. nutritional contribution, such as protein, in relation to feed requirements and availability). The scenarios revealed trade-offs between the different nutrients. When fatty acids were maximised, supply of minerals and vitamins (e.g. iron, zinc, and vitamin A) was reduced. When maximising the three minerals calcium, iron, and zinc, mainly the fatty acids showed a substantial decrease. Also increased supply of vitamins came at the expense of fatty acids. Overall, these trade-offs per scenario were most pronounced for the fatty acids DHA and EPA, whereas for 
calcium, protein, vitamins $A$ and $B_{12}$, and zinc, the signals were less strong. These results emphasise the potential nutritional contributions of ASF, which are embedded within a balanced diet.

No alternative ASF scenario of any country was able to meet the protein contribution of the ASF recommended in the original FBDG diets (Figure 2). The maximum achievable protein contribution based on LCB (Scenario MaxProt) ranged from $15.9 \mathrm{~g}$ protein per capita per day (corresponding to $27 \%$ of total protein contribution of the original FBDG; Malta) to 38.9 g protein per capita per day ( $47 \%$ of total protein contribution of the original FBDG; Switzerland). While the Netherlands could provide $21.6 \mathrm{~g}$ protein per capita per day (22\% of total protein contribution of the original FBDG), Sweden reached $25.4 \mathrm{~g}$ (30\% of total protein contribution of the original FBDG), and Bulgaria reached $37.4 \mathrm{~g}$ ( $40 \%$ of total protein contribution of the original FBDG). With this, all countries could provide at least one third of the average daily protein needs (EFSA, 2017; WHO, 2007).

In some scenarios and countries, ALA, calcium, zinc, vitamin A, and vitamin $\mathrm{B}_{12}$ of the ASF of the original recommendations could be met, while DHA and EPA were always deficient (Figure 2). For Malta, no nutrient contribution at the same level as in the original FBDG could be achieved. For Bulgaria, all scenarios were able to cover ALA, calcium, zinc, and the vitamins A and $B_{12}$ intake. In the Netherlands, only vitamin A reached the original contribution. Moreover, in Sweden ALA, calcium, and vitamin A could be fulfilled, while others, such as DHA and EPA, were strongly deficient. Switzerland met the ASF-related nutrient contributions of the FBDG diets the best, with protein and iron being slightly deficient, and DHA and EPA clearly deficient.
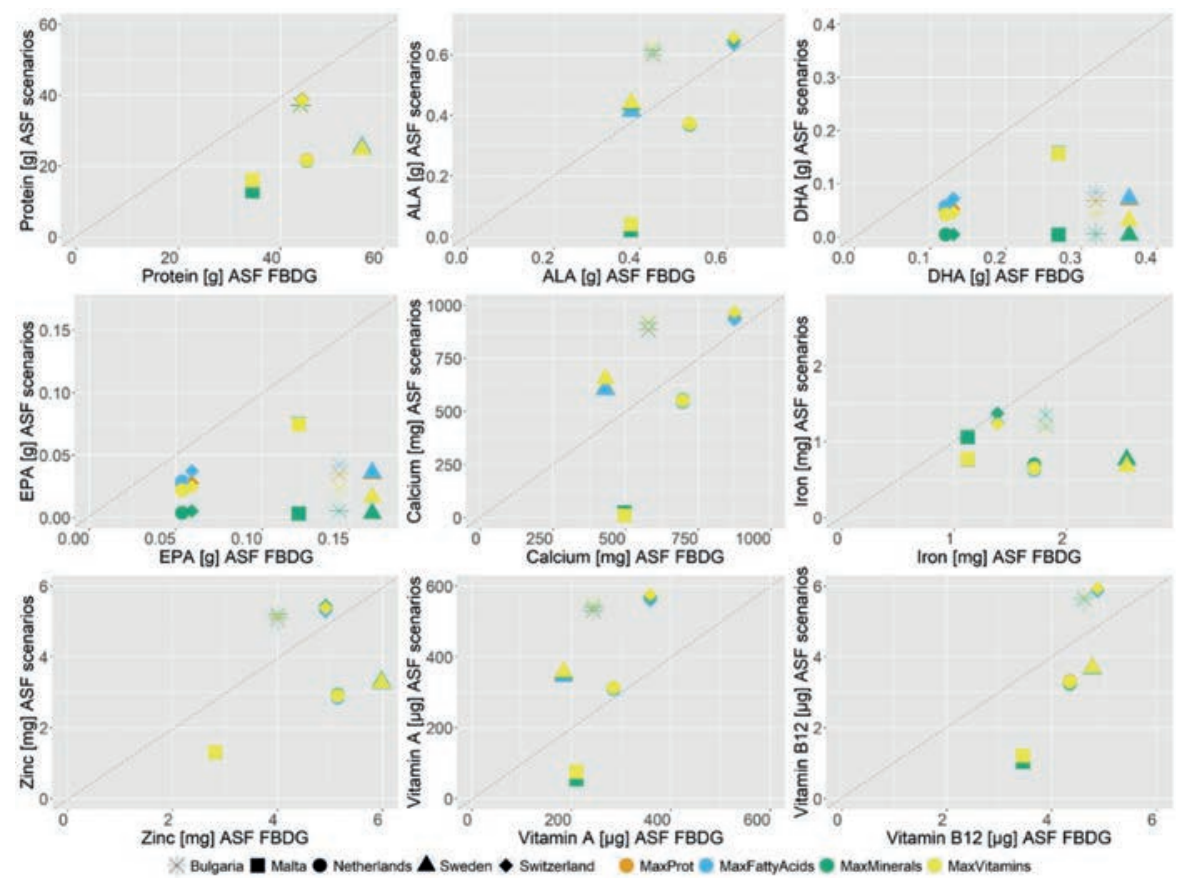

Figure 2. Nutrients of the animal-source foods (ASF) in the original food-based dietary guidelines (FBDG), $x$-axis, plotted against nutrients of the ASF in the scenarios (colour) and countries (shape), $y$-axis, per capita per day. The diagonal line indicates equal nutritional contributions in the scenarios and FBDGs. For values in the upper triangle, nutrient contribution of the scenarios exceeds those of the FBDGs, and in the lower triangle, nutrient contribution of the scenarios is lower than those of the FBDGs. Horizontal differences: countries, vertical differences: scenarios. 
The differences in nutritional composition stem from changes in the composition of the ASF in the scenarios (Figure 3). Compared to the scenario MaxProt, increases in fatty acids were mainly reached with increased fish, and for Bulgaria and Switzerland increased pork. When minerals were in focus, eggs were substantially increased, mainly at the expense of pork and fish. Moreover, focusing on vitamins $\mathrm{A}$ and $\mathrm{B}_{12}$ increased milk and the associated meat production.

Resource endowments largely drove the final composition of ASF. Milk and beef supply was mostly driven by available grass resources, which led to minimal supply of milk and beef in Malta, where grass resources are scarce. Grass resources were the main driver for milk production in the countries Bulgaria, the Netherlands, Sweden, and Switzerland, and thereby contributed most to total protein. Thus, although milk recommendations in the original FBDGs were substantial, these were in line with ASF from LCB. On the contrary, chicken and fish recommendations could never be met, while pork and eggs recommendations could be met for Malta, in some scenarios.

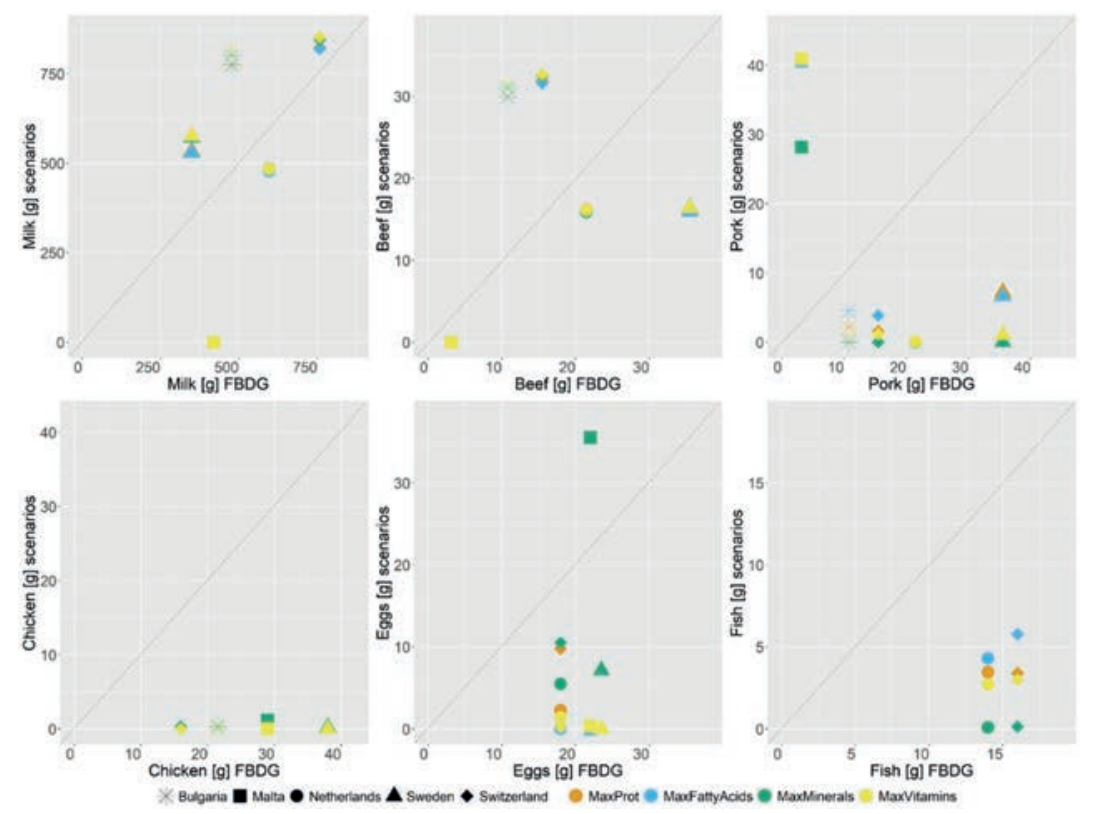

Figure 3. Quantities of the animal-source foods (ASF) in the original food-based dietary guidelines (FBDG), $x$-axis, plotted against quantities of the ASF in the scenarios (colour) and countries (shape), y-axis, per capita per day. The diagonal line indicates equal nutritional contributions in the scenarios and FBDGs. For values in the upper triangle, quantities of the scenarios exceeds those of the FBDGs, and in the lower triangle, quantities of the scenarios are lower than those of the FBDGs. Horizontal differences: countries, vertical differences: scenarios.

All scenarios showed a strong decrease in GWP as compared to the ASF of the original FBDGs (Figure 4). In Bulgaria, around 75\% less greenhouse gas emissions could be emitted. Malta showed the strongest potential decrease of $95 \%$ for GWP, while for the Netherlands, GWP could decreased by around $67 \%$. In Sweden, GWP of the ASF of the scenarios was around $60 \%$ lower than of the ASF in the original FBDGs, and scenarios for Switzerland showed a potential decrease of $77 \%$. Notably, these reductions in GWP were paralleled by substantially lower ASF protein supply. A 1\% decrease in ASF protein coincided with a decrease in GWP of 5\% (Bulgaria), 1.8\% (Malta), 1.3\% (Netherlands), $1.1 \%$ (Sweden), and 6.6\% (Switzerland). Thus, especially for Bulgaria and Switzerland, substantial 
reductions of GWP can be achieved with relatively moderate reductions in ASF protein. Generally, the lower GWP values of the scenarios can be linked to three causes: firstly, to the total decrease of ASF, secondly, to the change in ASF composition, and thirdly, to changes in the feeding regime in the different scenarios. Next to GWP, cropland use of the ASF in the scenarios was reduced to a minimum, which resulted from the assumption that only LCB is fed.

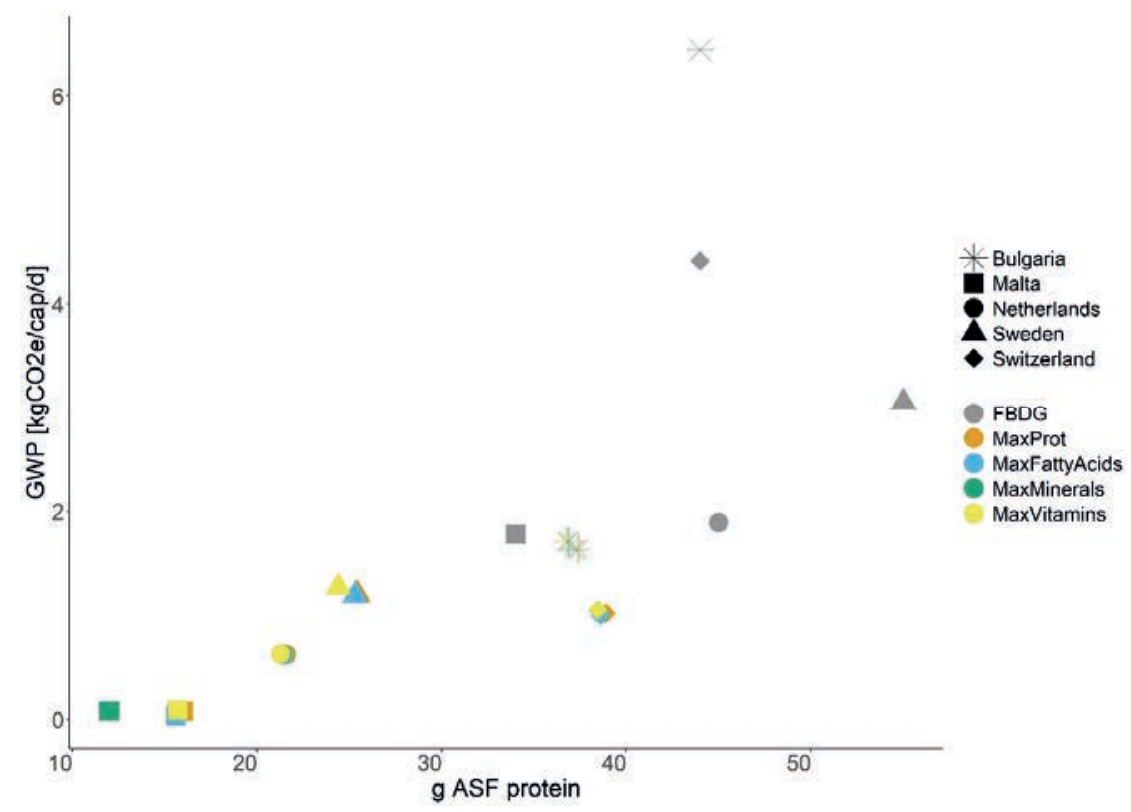

Figure 4. Global warming potential (GWP) and $g$ of animal-source food (ASF) protein per original food-based dietary guideline (FBDG) and scenarios (colour) and countries (shape).

\section{Discussion}

While national FBDGs provide important orientation for healthy dietary habits, they often do not consider resource use and associated environmental impacts in their formulation. The five national FBDGs assessed in this study recommended substantial amounts of ASF, partly contributing more protein than needed. ASF could thus be reduced to some extent while still meeting protein requirements. Our results clearly demonstrated the benefits in terms of resource use and greenhouse gas emissions of reduced ASF in the FBDGs, and showed that ASF from LCB could cover a substantial part of the protein needs of the studied populations. Our quantitative estimates for the potential of ASF under different nutritional foci can thus directly contribute to a discussion on recommended targets for the inclusion of ASF produced under circularity principles. Such ASF could contribute between $22 \%$ (Netherlands) and $47 \%$ (Switzerland) of total protein contributions of the original FBDGs. Clearly, parallel to the indicated reduction of ASF consumption, moving towards a more circular food system would require substantial adjustments of ASF production. Only by consistent transformations of food systems in this regard, the estimated environmental and resource use efficiency improvements can be reached. 


\section{4 | Chapter 5}

In line with our findings, the study by Springmann et al. (2020) found that ASF recommendations in most FBDGs are too high both from a human health and environmental impact perspective. From a human health perspective, it is assumed that ASF can be reduced to a minimum (Springmann et al., 2018b). Nevertheless, from a resource use perspective some animals can contribute to global food security. Van Zanten et al. (2018) defined a land boundary for sustainable livestock consumption and thereby concluded that on a global average, 9-23 $\mathrm{g}$ of protein per capita, which covers around one third of the daily protein requirement, could be derived from ASF solely based on LCB. The recommended amounts of protein from ASF in the five FBDGs assessed however ranged from 34 to

56 g. Remarkably, this amount could almost cover protein needs without considering plant-based foods. Cultural aspects can possibly explain these relatively high shares of ASF: taking the example of Switzerland, cattle played (and plays) an important role in converting grass resources from marginal areas into valuable ASF. Consequently, consumption of dairy - and the associated meats and fats - proved an essential source for protein and fats for the Swiss population. However, currently, grass resources are partly grown on land that could be used for human food consumption, and not all of this is temporary grassland with an agronomic function in crop rotations. Thereby, part of these grasslands come with higher opportunity costs. ASF based on LCB would thus use less grass resources also in Switzerland. In sum, environmental and nutritional concerns raise the need to revise the Swiss dietary recommendations towards less ASF. This then also allows to meet nutritionally consistent recommendations with LCB-sourced ASF only, which brings environmental and human health aspects in line. The same line of thought applies to other ASF as well as other countries, which confirms the urge to look at food systems redesign at a more regional level (Springmann et al., 2018b).

Next to national FBDGs, Willett et al. (2019) proposed a new healthy reference diet and related it to the food systems' share of the planetary boundaries. However, first, suitability of resources and resulting competition between feed and food was not considered. Therefore, Van Selm et al. (2021) tested whether ASF recommended by Willett et al. (2019) could be met with ASF from LCB, finding that both meeting the total protein contribution as well as meeting the proposed composition of ASF is challenging. Second, the proposed healthy reference diet lacks translation to local contexts. Comparisons with national FBDGs are a starting point for this translation. In terms of ASF protein from total protein of the recommendations, the FBDGs employed here had higher values, doubling or even tripling this share.

The four scenarios showed that in order to fulfil a diverse set of nutritional requirements, diversity in ASF consumption is key. Fish and seafood substantially contribute to the chosen omega-3 fatty acids. In some countries, this even led to an increase in pork production, with the by-products being used as feed for salmon (Bulgaria and Switzerland) (cf. Van Hal et al. (2019a)). When focusing on minerals, i.e. zinc, calcium, and iron, eggs and milk production were increased, with the associated meats being increased as well. Further, also when the two vitamins A and B12 were in focus, milk and the associated meat showed a slight increase. In the selected FBDGs, the nutritional function of ASF beyond protein supply was rarely mentioned in the recommendations, and thus, the reasoning behind the recommended quantities of ASF was not further clarified. 
For chicken, results diverged most between our scenarios and FBDG recommendations. While chicken is the meat type most often recommended in FBDGs (Herforth et al., 2019), it was rarely selected in the proposed scenarios, independent of the nutritional focus. On the one hand, chicken is often promoted as a relatively sustainable source of meat due to its favourable feed conversion ratio, resulting in efficient production and low environmental impact intensities per $\mathrm{kg}$ of product (Frehner et al., 2020). However, the high efficiency of chicken comes with a downside, namely the required high quality of feed that cannot be provided by standard circular feed production methods (Van Hal et al., 2019a). As a consequence, currently widespread chicken breeds are not able to feed on lower quality feedstuffs (to which part of LCB belong), and are hence not competitive in scenarios with LCB. In current production systems, feed for chicken is often of high quality, and its production competes directly or indirectly with human food production (Mottet et al., 2017). Notably, results could look different if e.g. GWP would be considered in the scenario definition process, which might favour chicken over other animal production systems. On the other hand, chicken disposes of a more favourable nutritional profile than other types of meat. In several epidemiological studies, no correlation with increased risk for non-communicable diseases was found for chicken, while it was found for red and processed meat types (Cocate et al., 2015; Etemadi et al., 2017).

Geographical circumstances shape the availability of locally available LCB. Here, we employed a national geographical scope, and did not allow trade of LCB. This led to large imbalances between countries regarding available LCB, and thus regarding available ASF. This imbalance was particularly pronounced for Malta, where available LCB was so low that a large nutritional gap resulted. Previous assessments of circular food systems took a global or regional perspective, and assumed that within these geographical contexts, part of the produced LCB can be traded freely (Van Hal et al., 2019a). Thus, countries with low levels of LCB could import LCB from other countries, based on the assumption of an equal distribution of LCB across the geographical scope assessed. Moreover, not only LCB availability is determined by the geographical level of assessment, but also the available share of landings from fisheries. Sustainable landings from fisheries could be an important ASF source in circular food systems. Both LCB as well as fish landings might not be ideally allocated in the country they are produced. It is thus important to investigate suitable and equitable distribution mechanisms of LCB, ASF, and fish landings. Thereby, the consequences and impacts of distribution have to be weighted against the benefits that occur when resources are allocated optimally across larger scales.

We point towards potential future improvements of our approach. First, we solely varied ASF in FBDGs, while keeping plant-based food constant. However, the proposed reductions in ASF would need to be compensated with increased or specifically diversified plant-based food, in order to compensate for the reduced nutritional value. For this, a land use model is needed that includes both plant-based food and ASF, while capturing resource use efficiency as well as flows between the different production systems.

Second, we acknowledge that environmental sustainability encompasses much more than GWP and land use. The production of our food affects the environment in many ways, by e.g. altering the global nutrient cycles, adverse impacts on biodiversity, and fostering soil erosion. Moreover, the environment is only one dimension of total sustainability as well; social and economic impacts of 


\section{6 | Chapter 5}

food production and human health implications of our food consumption complete the picture of sustainability. Although we only considered the two environmental indicators, our proposed scenarios would most likely affect many other dimensions of sustainability. For example, nitrogen surplus could be reduced substantially, resulting from the reduction in animal farming and omission of imported feed. This omission would lead to a reduction of the whole nutrient throughput in the system and thus potentials for losses are smaller. Further research could thus, for example, focus on implications on nutrient flows and soil health of following such scenarios, as well as economic and social consequences.

In conclusion, the proposed approach can transparently contribute to a discussion on recommended targets for the inclusion of ASF produced under circularity principles. Although the ASF recommended in the FBDGs of Bulgaria, Malta, the Netherlands, Sweden, and Switzerland is neither in its composition nor in its total nutritional value achievable with ASF from LCB, $45-88 \%$ of the protein from ASF recommendations could be met with the proposed circularity principle. This result comes with major implications for the five national FBDGs assessed, and probably applies to many others in Western countries (Springmann et al., 2020). In fact, to make the dietary guidelines of these countries compatible with principles from circular food systems as well as protein requirements, ASF recommendations would need to be substantially reduced. Such a reduction would furthermore lead to a substantial reduction in GWP. Clarity regarding the nutritional function of the recommended ASF in the diet could help to target the ASF composition, and to decide how to allocate LCB resources best.

\section{Acknowledgements}

We thank A. Hayer (Swiss Society for Nutrition SSN) for inputs regarding interpretation of the Swiss dietary guidelines. The contribution of AF, AM, and CS to this work was supported by the Swiss National Science Foundation (National Research Project 69: "Sustainable and healthy diets: Tradeoffs and synergies", project 4069- 166765). 


\section{Chapter 6}

\section{The compatibility of animal-source food and circularity in healthy European diets}

B. Van Selm ${ }^{1,2}$, A. Frehner ${ }^{1,3}$, I.J.M. De Boer ${ }^{1}$, O. van Hal', R. Hijbeek ${ }^{2}$, M.K. Van Ittersum², E.F. Talsma $^{4}$, J.-P. Lesschen ${ }^{5}$, C.M.J. Hendriks ${ }^{5}$, M. Herrero ${ }^{6}$, H.H.E. Van Zanten ${ }^{7}$

\footnotetext{
${ }^{1}$ Animal Production Systems group, Wageningen University \& Research, Wageningen, the Netherlands

2Plant Production Systems group, Wageningen University \& Research, Wageningen, the Netherlands

${ }^{3}$ Department of Socioeconomics, Research Institute of Organic Agriculture FiBL, Frick, Switzerland

${ }^{4}$ Division of Human Nutrition and Health, Wageningen University \& Research, Wageningen, the Netherlands

${ }^{5}$ Wageningen Environmental Research, Wageningen University \& Research, Wageningen, the Netherlands

${ }^{6}$ Commonwealth Scientific and Industrial Research Organisation, Australia

${ }^{7}$ Farming Systems Ecology group, Wageningen University \& Research, Wageningen, the Netherlands
}

Under review 


\section{8 | Chapter 6}

\section{Abstract}

Several dietary guidelines are developed that propose limiting the intake of animal protein to stay within planetary boundaries and improve human health. Simultaneously, circular food systems are receiving significant attention in the European Union as an option to improve the current food system. In a circular system, animals are solely fed with low-opportunity-cost-biomass, resulting in substantially fewer animals and reduced supply of animal-source nutrients to humans. We assessed whether this circularity principle within the EU-28 is compatible with the recommended animalsource food consumption in healthy and environmentally friendly dietary guidelines such as the EAT-Lancet dietary guidelines. Our results show that the overall quantity of animal-source protein in EAT-Lancet dietary guidelines can be met, but that the precise levels of inclusion of different animal-source foods in such a diet cannot be achieved. The EAT-Lancet guidelines recommend larger quantities of chicken meat over beef and pork while a circular food system produces mainly milk, dairy-beef, and pork. All three circularity diets outperform the EAT-Lancet diet in nutritional value while reducing greenhouse gas emissions (up-to 31\%) and arable land use (up-to 42\%). Careful consideration of the permissible substitutability between animal-source foods is urgently needed to define the role of animal products in circular human diets. In this way the consumption of animal products - based on the circularity principle of only feeding animals with low-opportunity-cost biomass - benefits both human health and the environment. 


\section{Main}

The global food system is responsible for about a quarter of all human-induced greenhouse gas (GHG) emissions, one third of global terrestrial acidification, the majority of global eutrophication, and is occupying $40 \%$ of the world's ice and desert-free land (Foley et al., 2011; Godfray et al., 2010). The global trend of a growing affluent population, thereby shifting diets towards resource-intensive foods (e.g. meat), is raising concerns that the Earth's biophysical limits will be exceeded (Godfray et al., 2018). To halt this progression, numerous healthy and environmentally-friendly dietary guidelines have been proposed (Eme et al., 2019; Springmann et al., 2018b; Willett et al., 2019). Such dietary guidelines aim to reduce environmental impacts (e.g. GHG emissions, deforestation, eutrophication and biodiversity loss) while simultaneously reducing the risk of non-communicable diseases (e.g. cardiovascular disease, colorectal cancer and type-2 diabetes) (Godfray et al., 2018; Springmann et al., 2018b). One prominent example of a healthy and environmentally sustainable dietary guidelines is the one of the EAT-Lancet commission. Compared to conventional diets consumed in the European Union (EU-28) this EAT-Lancet dietary guidelines contain increased quantities of plant-sourced food, such as vegetables, legumes and nuts, and decreased quantities of animal-source food, such as meat, milk, eggs, and fish (Willett et al., 2019).

The foods that European societies consume, and the way they are produced, contribute substantially to destabilizing several planetary boundaries (Campbell et al., 2017; Steffen et al., 2015). Although there is general consensus that the consumption of animal-source food in the EU-28 should decrease, there is no consensus about the degree of reduction of animal-source food to achieve healthy and environmentally sustainable diets (Frehner et al., 2020). Some studies suggest that it would be best for the planet if we would consume plant-sourced foods only (e.g. Poore and Nemecek, 2018), while others show that farm animals reared under a circular paradigm can play a crucial role in feeding humanity (Röös et al., 2017; Schader et al., 2015; Van Hal et al., 2019a; Van Kernebeek et al., 2016; Van Zanten et al., 2018). Circular food systems aim to optimally utilise resources by prioritising arable land to produce plant biomass for human consumption, thus avoiding feed-food competition (De Boer and Van Ittersum, 2018; Van Zanten et al., 2019). Currently about $40 \%$ of our global arable land is used to produce high-quality feed for farm animals, which to a large extent is human-edible (Mottet et al., 2017). From a resource-efficiency point of view, farm animals, instead, could be fed low-opportunity-cost-biomass (LCB), which includes co-products from the food industry (e.g. wheat middling's or slaughter waste from farm animals), food waste, and grassland resources (Van Hal et al., 2019a). In this case, the resource use efficiency of the farm animals is increased which has potential to reduce the environmental impacts (Schader et al., 2014; Van Kernebeek et al., 2016).

Our aim was to assess whether adhering to the circularity principle of feeding LCB to farm animals within the EU-28 is compatible with the recommended animal-source food consumption in healthy and environmentally friendly dietary guidelines. We took a reference diet derived from the EATLancet dietary guidelines as an example of a future healthy diet of which the environmental impacts of the food system were kept within the safe operating space of the planetary boundaries' framework (Willett et al., 2019). To adhere to circularity principles applied to the EU-28, animals were fed coproducts and food waste resulting from the plant-sourced fraction of EAT-Lancet diet (Figure 1). In 
addition, grassland resources and slaughter by-products from farm animals could be used as animal feed. A resources allocation model was used to distribute the LCB among animal production systems (dairy, beef, pigs, broilers, layers, salmon and tilapia) to maximize protein production while respecting recommended animal-source food intake levels of the EAT-Lancet dietary guidelines as well as land use and GHG emission boundaries. Crop and animal production systems in the EU-28 were based on current management and yields (i.e. $\mathrm{kg}$ per hectare or $\mathrm{kg}$ per animal). Nutrient adequacy of the EAT-Lancet dietary guidelines was assessed against the European Food Safety Agency (EFSA) human nutrient intake requirements (EFSA, 2017). Four scenarios were investigated (Table 1), firstly an EAT-Lancet reference scenario which represented the EAT-Lancet dietary guidelines in their current form (EL Reference). Secondly, a healthier whole-grain diet with a fixed composition of animal-source food (EL Circular Wholegrain Fixed). Thirdly, a refined grain diet with a fixed composition of animal-source food (current grain consumption is dominated by refined grains and consuming wholegrains results in less by-products from cereal processing and can therefore strongly affect the role of animals when adopting circularity principles, (van Hal et al., 2019a) (EL Circular Refined-grain Fixed). Lastly, a wholegrain diet with an unrestrained quantity of animal-source food to demonstrate the production potential of animals fed LCB (EL Circular Wholegrain Potential). Scenarios two to four provide insight into the debate about which and how many animals to keep in a circular food system and the trade-offs and synergies with health recommendations.

Table I. Overview of scenarios employed.

\begin{tabular}{llllc}
\hline & Diet type & \multicolumn{2}{c}{ Animal-source food composition } \\
& Wholegrain & Refined grain & Fixed & Free \\
\hline EL Reference & $\mathrm{X}$ & $\mathrm{X}$ & \\
EL Circular Wholegrain Fixed & $\mathrm{X}$ & $\mathrm{X}$ & \\
EL Circular Refined-grain Fixed & & $\mathrm{X}$ & $\mathrm{X}$ & $\mathrm{X}$ \\
EL Circular Wholegrain Potential & $\mathrm{X}$ & & & \\
\hline
\end{tabular}




\section{Results}

\section{I Animal-source food supply from circular food systems}

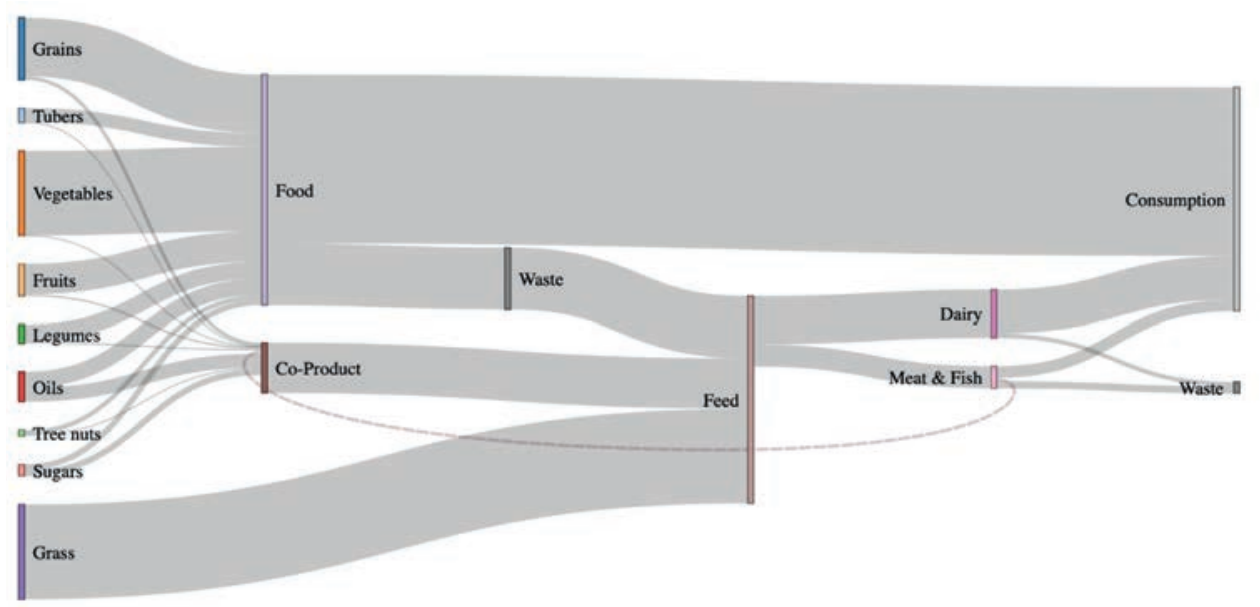

Figure I. Framework to assess the supply of animal-source food from animals fed LCB. Example shown represents the EL Circular Wholegrain Fixed scenario. All flows are in fresh matter except grass which is in dry matter.

Our analysis revealed that animals exclusively fed LCB were unable to provide the combination of meat, milk, eggs and fish recommended in the EAT-Lancet dietary guidelines, largely due to an insufficient quantity of high quality LCB. In total, the reference diet derived from the EAT-Lancet dietary guidelines contained 71 grams of meat and fish, 250 grams of milk and 13 grams of eggs per capita per day. It was, nevertheless, possible to fulfil these recommendations by adjusting the share of meat and fish while respecting the healthy range. The reference value for pork, for example, is 7 grams, while the healthy range is 0-14 grams of pork per capita per day.

In the EL Circular Wholegrain Fixed scenario, recommended quantities of milk and fish could be met while meat and eggs were $5 \%$ and $92 \%$ short of meeting the recommended intake in EAT-Lancet dietary guidelines. In the EL Circular Refined-grain Fixed scenario, the recommended quantities of meat, milk, eggs and fish could be met due to the additional LCB available from the refining of grains (e.g. wheat bran). However, adjusting the shares of meat and fish was still required. Compared to the EL Circular Wholegrain Fixed, the EL Circular Refined-grain Fixed scenario could produce more poultry meat (4 vs. 2 grams of poultry meat) and meet the recommended intake of eggs in the EATLancet dietary guidelines (13 eggs per capita per day). From a health externalities perspective, the consumption of poultry meat is preferred over the consumption of beef and pork (Willett et al., 2019). Broilers and laying hens, however, were limited in their ability to upcycle all types of LCB and mainly required the co-products from refined grains. This creates a trade-off between consuming healthy whole grains or producing healthy white poultry meat and eggs.

The EL Circular Wholegrain Potential scenario showed the optimal allocation of LCB (in terms of maximising protein production) to different animals (Figure 2). This scenario resulted in an increase in pork production (to 40 grams per capita per day) due to a pig's ability to convert low quality co- 


\section{2 | Chapter 6}

products and food waste into animal-source food. Milk production also increased (to 563 grams per capita per day) as dairy cattle are efficient converters of LCB (especially grassland) to protein. Increased milk production increased the supply of cull cows which produced additional beef. The increase of pork and milk was at the expense of poultry and fish production, thus showing a tradeoff between optimally utilising LCB and producing the preferred white meat.

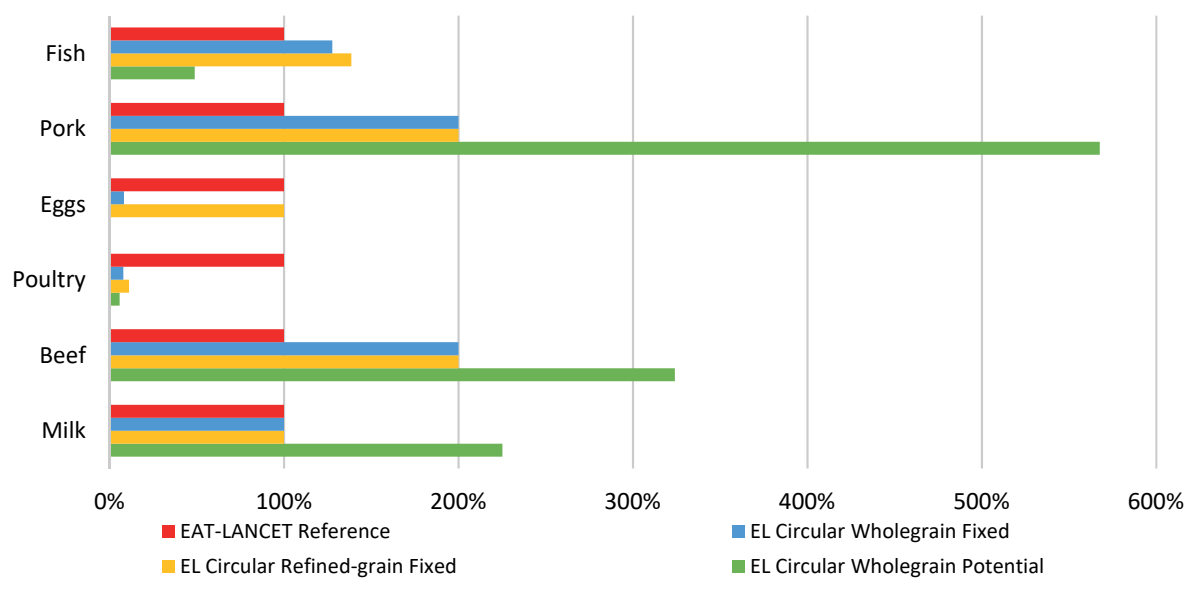

Figure 2. Animal-source food production from the three EL Circular scenarios. 100\% is equal to the recommended intake in EATLancet dietary guidelines, i.e. 28 grams of fish, 7 grams of pork, 13 grams of eggs, 29 grams of poultry meat, 7 grams of beef and 250 grams of milk.

\subsection{Human nutrient supply from circular food systems}

Our results showed that the EL Circular Wholegrain Fixed, EL Circular Refined-grain Fixed scenarios and the EAT-Lancet reference did not meet zinc, calcium, vitamin $B_{12}$ average nutrient requirements of the human population set out by EFSA (Figure 3; Appendix E1). Notably, the EATLancet reference also fails to meet EPA/DHA average nutrient requirements (Figure 3). The EL Circular Wholegrain Potential did meet the calcium and vitamin $B_{12}$ requirements but not zinc however, largely due to an increase in milk production (250 grams vs 563 grams). For all nutrients except EPA/DHA (due to less fish), nutrient supply was greatest in the EL Circular Wholegrain Potential scenario. Besides calcium, all three circularity diets outperformed the EAT-Lancet diet on available nutrients. 


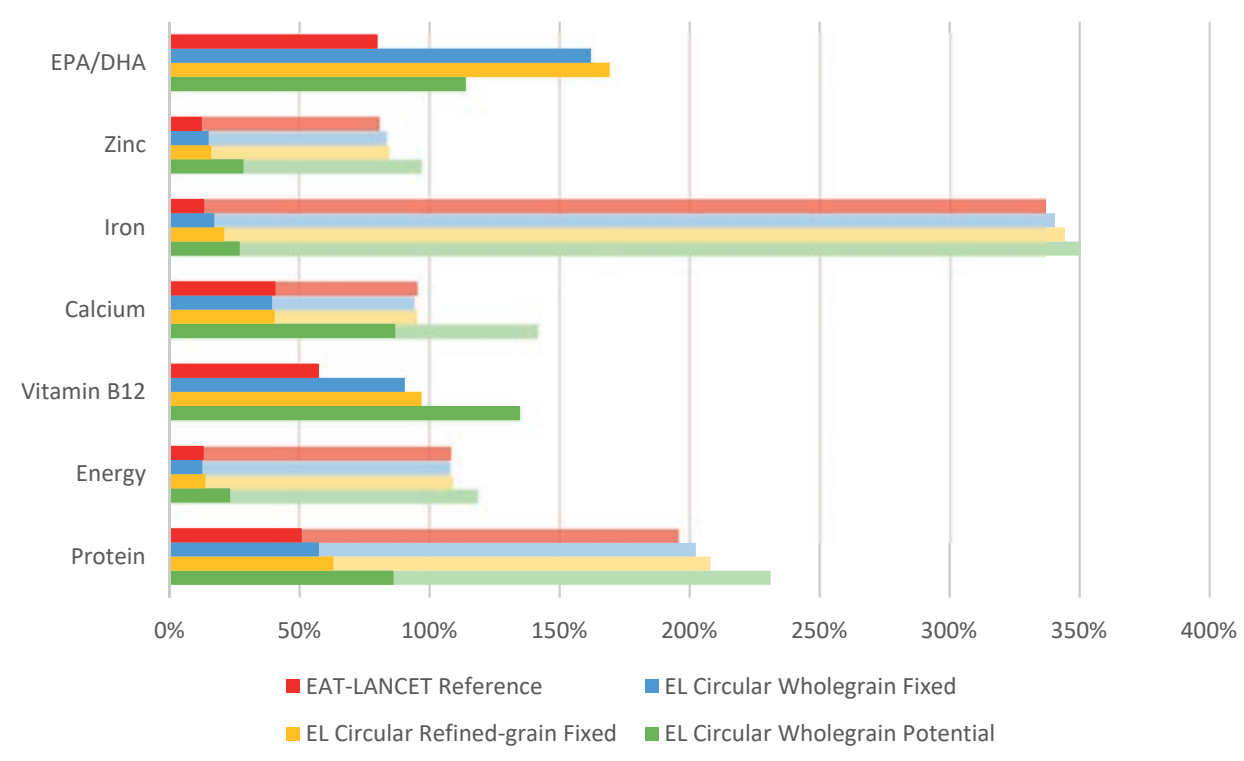

Figure 3. Nutrients available in all EL Circular scenarios and the EAT-Lancet reference diet scenario. $100 \%$ is equal to the daily average nutrient requirements outlined by EFSA (Appendix EI). Nutrients are shown from animal-source food (solid fraction) and plant-sourced food (shaded fraction). Total daily dietary nutrient supply includes the solid and unshaded fractions. EPA: Eicosapentaenoic acid. DHA: Docosahexaenoic acid.

\subsection{Greenhouse gas emissions and land use impacts of circular food systems}

Overall, GHG emissions were 31\% and 28\% lower in the EL Circular Wholegrain Fixed and EL Circular Refined-grain Fixed compared to the EAT-Lancet reference scenario. The reduction in emissions was due to the avoided emissions related to the production of animal feed (e.g. nitrous oxide $\left(\mathrm{N}_{2} \mathrm{O}\right)$ from nitrogen fertilisation) and the EL Circular Wholegrain Fixed scenario requiring less grain production (i.e. more grain was destined for human consumption, due to no refining).

Figure 4 shows GHG emissions and animal-source food protein produced from all three EL circular scenarios and the EAT-Lancet reference diet. Higher quantities of animal-source food (and therefore protein) were produced in the EL Circular Wholegrain Potential and lower quantities in the EL Circular Wholegrain Fixed scenarios which influenced GHG emissions (Figure 4). The EL circular scenarios include a default GHG emission value (according to the IPCC tier 2 approach) and a range of uncertainty to reflect the uncertainty in GHG emissions (Appendix E1). Optimally utilising LCB (to maximise protein production from animal-source food) in the EL Circular Wholegrain Potential scenario increased default GHG emissions to $477 \mathrm{~kg} \mathrm{CO}_{2} \mathrm{e}$ per capita per year from $367 \mathrm{~kg} \mathrm{CO} 2 \mathrm{e}$ per capita per year in the EL Circular Refined-grain Fixed scenario (largely due to an increase in milk and pork production, Appendix E1). The default GHG emission values of all scenarios were within the safe operating space of the planetary boundaries' framework $\left(511 \mathrm{~kg} \mathrm{CO}_{2} \mathrm{e}\right.$ for food production per capita per year; Willett et al., 2019). In all EL circular scenarios, the upper limit to the range of uncertainty was beyond the safe operating space. 


\section{$84 \mid$ Chapter 6}

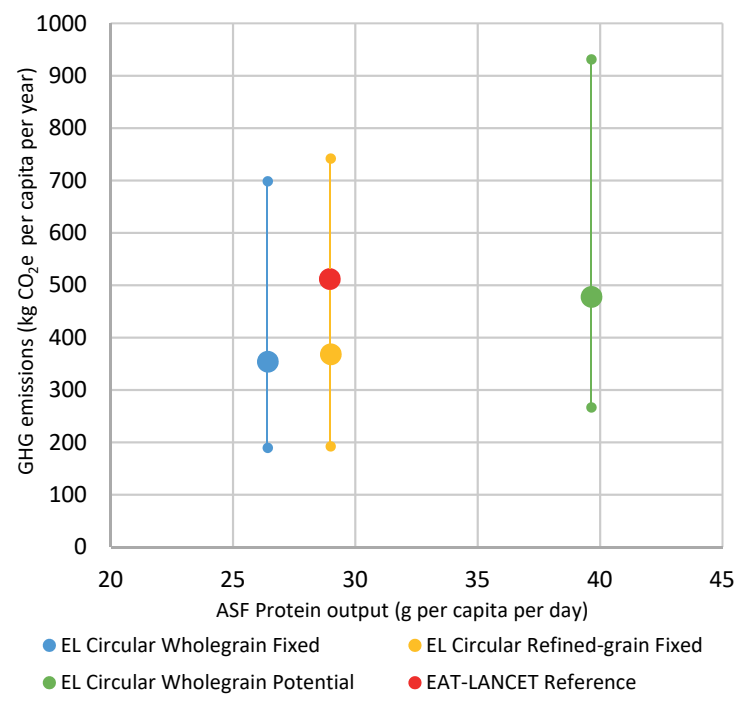

Figure 4. $\mathrm{GHG}$ emissions ( $\mathrm{kg} \mathrm{CO}$ e capita per year) and protein output from animal-source food (ASF) (g capita per day) of the EL Circular scenarios compared to the GHG emission results calculated by the EAT-Lancet commission for their reference diet (red dot). Range indicates uncertainty in GHG emissions.

Overall, cropland use was lower in all EL circular scenarios compared to the EAT-Lancet reference diet. However, it was important to note that the EAT-Lancet reference scenario was a global land use average, while the EL circular scenarios were based on EU land use. Further, utilising cropland to produce animal feed also led to an increase in land use in the EAT-Lancet reference scenario. Cropland use was lowest in the EL Circular Wholegrain Fixed and EL Circular Wholegrain Potential scenarios due to the use of wholegrains requiring less land (i.e. less co-products from wheat results in less land required), though differences with using refined grain were marginal. Grassland use of the EL Circular Wholegrain Fixed and EL Circular Refined-grain Fixed were similar while the EL Circular Wholegrain Potential scenario resulted in a higher grassland use, as the use of grassland resources was increased for milk production (Figure 5).

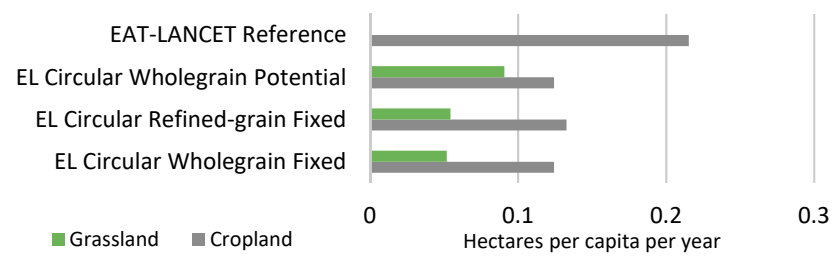

Figure 5. Cropland use (ha per capita per year) from the EAT-Lancet reference diet (which is an estimate for the entire globe, calculated by the EAT-Lancet commission) compared to cropland and grassland use in the EU-28 of the three circular scenarios.

The milk and beef production in circular food systems was highly dependent on the availability of grassland. Variation exists in the data of quantity and quality of current grassland in the EU-28 depending on the study and definition of grassland (i.e. between managed and natural grassland) and available data sources. We compared the animal-source food output (e.g. milk) of the EL Circular Wholegrain Potential scenario with different areas of managed grassland resulting from 
three different studies/models (Haberl et al., 2007; Plutzar et al., 2016; Velthof et al., 2009). Milk production and beef (from dairy cattle) ranged, respectively, from 326 to 780 and 11 to 37 grams per capita per day (Appendix E1). Including natural grasslands could further increase the output of animal-source food.

\section{Discussion}

Our results show that the overall quantity of animal-source protein in EAT-Lancet dietary guidelines can be met, but that the precise levels of inclusion of different animal-source foods in such a diet cannot be achieved by only feeding LCB to animals. The extent to which the recommended quantities of animal-source food could be met largely depended on the availability of the LCB. The EL Circular Wholegrain Fixed scenario versus the EL Circular Refined-grain Fixed scenario revealed that the role animals can play in circular food systems will be narrowed as we move towards healthier consumption of plant-sourced foods. With today's food consumption patterns, several food groups are consumed in highly processed forms, resulting in additional by-products on the one hand, but increasing the risk for non-communicable diseases on the other hand (Elizabeth et al., 2020). The example employed here, wheat, results in by-products such as wheat bran and wheat germ if wheat is consumed in a refined manner. If it is however consumed as whole grain, as recommended in the EAT-Lancet dietary guidelines, no by-products occur. Potatoes, vegetables, and fruits would be other examples; if potatoes for example are industrially processed, potato peels can be collected and thereafter used as animal feed.

Although dietary guidelines could not be met, it was possible to meet the nutrient recommendations when the EAT-Lancet diet restrictions were removed (i.e. the EL Circular Wholegrain Potential scenario). Comparing the two EL circular fixed scenarios with the EL Circular Wholegrain Potential scenario showed that some animal species were more efficient at upcycling LCB (e.g. dairy cattle and pigs) than others (e.g. poultry). Grass resources for example were utilised most efficiently by dairy cattle as ruminants are well adapted to value this feed. Wet or fibrous food leftovers are used most efficiently by pigs that are known to have a high feed intake capacity. Milk, furthermore, includes relatively high amounts of calcium and beef and pork are high-quality sources of bioavailable vitamin $B_{12}$ and zinc (Murphy and Allen, 2003). In other words, each animal has its own unique capacity to convert LCB into specific nutrients. The nutrients provided by animals are of high bioavailability and some, such as vitamin $\mathrm{B}_{12}$ and the omega-3 fatty acids EPA and DHA are predominantly provided by animal-source foods and are almost absent in plant-source-foods (Murphy and Allen, 2003).

The circular scenarios and in particular the EL Circular Wholegrain Potential scenario showed that animals raised in a circular food system can play an essential role in providing nutrients. This is in line with earlier findings of for example Röös et al. (2017), Van Hal et al. (2019a), Van Zanten et al. (2018). None of those studies, however, assessed the importance of the dietary recommendations. Our results made clear that although the EL Circular Wholegrain Potential scenario met all nutrient recommendations except zinc, it exceeded the intake of beef and pork. Findings from cohort studies suggest that occurrence of several non-communicable diseases, such as cardiovascular disease, was 
associated with a relatively high intake of red meat, i.e. beef and pork (e.g. Etemadi et al. 2017). The higher recommended amounts of poultry as compared to beef in the EAT-Lancet reference diet was justified by the fact that poultry meat does not show associations with increased mortality, and poultry fat moreover disposes over a higher content of essential poly-unsaturated fatty acids (21\% vs. $4 \%$ ) (Willett et al., 2019). The above illustrates that although it is important to optimize essential nutrients from animal-source food, following upper limits of dietary guidelines is essential to avoid dietary related diseases.

Our results furthermore showed that when circularity principles were adopted, GHG emissions and land use were reduced compared to the EAT-Lancet diet, as feed-food competition was avoided. In Willett et al. (2019), broilers perform better than e.g. cattle meat from an environmental perspective, due to their favourable feed conversion ratio. However, their assessment is based on impact intensities (e.g. GHG emissions per $\mathrm{kg}$ of food product) calculated for the current system. Thereby, it was not considered whether the feed for broilers would also have been suitable as food for humans, or whether the area it was grown upon would have been suitable to grow food for humans. Our analysis clearly shows that as soon as we move towards a circular food system and hence restrict the role for animals to converting LCB, broilers cannot compete with e.g. cattle anymore. This does not mean that broilers cannot play a role in circular food systems, but it demonstrates that the broilers of today are less suited to convert LCB. This stresses the importance of adapting future breeding goals and feeding strategies towards their ability of utilizing LCB. This is essential as our results showed that animals can reduce their environmental impact (and the impact of the entire food system) if they increase their efficiency in converting LCB into healthy food.

Our study and model focused on feeding LCB, including food waste, to animals as a principle of circularity. The nutrient content of food waste was a weighted average based on the amount of food consumed in the human diet and the proportion wasted. By combining these products into one mix the feed value in terms of energy and protein of higher quality waste products (e.g. grains) is diluted by lower quality waste products (e.g. vegetables). Expanding the model employed to separate streams of food waste may increase the amount of animal-source food produced due to a greater availability of high quality LCB. Further expanding the optimisation model to include plant-sourced food production within the EAT-Lancet diet could offer additional opportunities to reduce GHG emissions and land use. In addition, more circularity principles could be captured, including returning nutrients in manure and crop residues to the soil. Applying alternative objective functions (e.g. minimising GHG emissions while meeting the nutrient requirements of the human diet) could also influence the animal production systems selected.

We would like to end by stressing the importance of future technologies (Herrero et al., 2020). In our work we followed the assumptions made by Willett et al. (2019). It was for example assumed that fossil energy was replaced by renewable energy sources, causing no $\mathrm{CO}_{2}$ emissions while crop and grass yields were based on current yields and management (obtained from the Miterra-Europe Model and Carlson et al. (2017)). In the coming decades, crop yields are expected to continue to increase due to ongoing technological developments (Fischer et al., 2014; Rijk et al., 2013). Increased crop yields would however not alter the main findings of our study, as this study focussed on the utilization choice of LCB, of which the amount is independent from crop yields. A change in crop 
yield would affect the environmental impacts of the remaining plant-sourced food needed to fulfil nutritional requirements. Increased crop yields may lead to less land use requirements per capita in each of the scenarios. The impacts of increased crop yields on GHG emissions are less clear. Achieving higher yields may need more nutrient inputs (especially nitrogen; Schils et al., 2018), with associated increases in GHG emissions per tonne product, but technological developments may also increase nutrient use efficiencies, playing a compensatory role.

\section{Conclusion}

We demonstrated that feeding low-opportunity-cost biomass to animals has the potential to reduce GHG emissions and land use. Our results showed that the quantity of animal-source protein in the EAT-Lancet dietary guidelines could be met, but that the precise animal-source food composition of the EAT-Lancet dietary guidelines could not be met by only feeding LCB to animals. Dietary guidelines recommend chicken meat over beef and pork while in a circular food system mainly milk, dairy-beef, and pork are produced. Careful consideration of the permissible substitutability between animal-sourced foods is urgently needed to define the role of animal products in the human diet. In this way the consumption of animal products - based on the circularity principle of only feeding animals with LCB - benefits both human health and the environment.

\section{Methods}

In this study we extended the resource allocation model developed by van Hal (2020) to include GHG emissions and land use. The model of van Hal (2020) allocates co-products and food waste resources from the EAT-Lancet example diet derived from the EAT-Lancet dietary guidelines (Willett et al., 2019), and grassland resources. We compared environmental impacts of the EATLancet diet with three EL circular scenarios. Each scenario varied based on the type of grain (wholegrain or refined grain) and the animal-sourced food composition. (Table 1).

\section{I Quantifying leftovers from the EAT-Lancet diet}

We took the example diet developed by the EAT-Lancet Commission (derived from the EAT-Lancet dietary guidelines) as a starting point for this study. To better reflect the EU diet, some adjustments were made to grain consumption (i.e. more wheat and less rice, total quantity of grain remained unchanged) based on FAOSTAT (FAOSTAT, 2018; Willett et al., 2019).

To calculate the amount (i.e. tonnes) and area of crop required and co-products available, reverse calculations were made using food consumption as a starting point. Quantities of co-products (e.g. wheat bran) from crops (e.g. wheat) were calculated using so-called technical conversion factors (FAO, 1996; Vellinga et al., 2013). In some scenarios grains were refined to increase the availability of co-products as animal feed and to better reflect current dietary habits (e.g. wheat bran, Table 1). Quantities of food waste were calculated using food waste fractions developed by Gustavsson et al. (2011). This process was performed for each of the EU-28 countries. 


\subsection{Resource allocation model}

The model of van Hal (2020) is a resource allocation model of the EU-28 developed in General Algebraic Modelling System (GAMS) version 30.3. The objective of the model is to maximise animal protein output from a given availability of animal feeds while meeting the nutritional requirements of the animals.

Animal systems include livestock (dairy, beef, pigs, broilers, and layers) and farmed fish (salmon and tilapia). The two fish systems are a proxy for a range of species with similar characteristics (e.g. rainbow trout for salmon). Livestock systems include three productivity levels (high, medium and low) while farmed fish only include a high productivity level. The model included the parent stocks (e.g. sow in pig system) and reproduction stocks (e.g. heifer in a dairy system) to account for the entire lifecycle of the animal. The nutritional requirements of livestock and farmed fish can be found in Supplement Material of van Hal (2020).

Livestock and farmed fish were exclusively fed co-products, food waste, grassland resources and animal by-products, referred to as LCB. In our model, co-products and animal by-products could be traded between EU-28 countries while food waste and grassland must be used in the country it is produced. The availability of co-products and food waste was set by the EL reference.

Thirty-five percent of the available food waste could be fed to animals as a wet feed, which is considered achievable if the feeding of food waste to animals were to be legalised ( $\mathrm{Zu}$ Ermgassen et al., 2016). Food waste could only be consumed by monogastric animals and fish due to food safety risks (Zu Ermgassen et al., 2016). The availability of European grassland was based on the MiterraEurope model (Velthof et al., 2009), and it was assumed grassland could only be consumed by ruminants. Our analysis only included managed grassland due to the uncertainty in quantity and quality of natural grasslands in Europe.

The availability of animal by-products was a fraction of the predicted live weight output of each livestock system (Supplement Material of van Hal (2020)). Cannibalism was prevented in livestock systems, in farmed fish systems cannibalism was allowed due to the species being a proxy of a range of species. This enabled intraspecies recycling of by-products from farmed fish, meaning farmed fish can consume by-products of the same species. The nutritional value of LCB for livestock was obtained from the Dutch animal feed board; known as the CVB system (Blok and Spek, 2016). While the nutritional value of LCB for farmed fish was obtained from the (IAFFD, 2018).

In addition to aquaculture, the model includes capture fisheries. Capture fisheries produced fish for human consumption and fish by-products (e.g. fish meal) which could be fed in the animal systems. Quantities of capture fisheries (i.e. harvested fish in tonnes of fresh fish) were limited to the maximum sustainable yield.

\subsection{GHG emissions and land use from animal-sourced food}

The resource allocation model developed by Van Hal et al. (2019a) was extended to include GHG emissions calculations. GHG emissions were based on Intergovernmental Panel on Climate Change (IPCC) and Dutch GHG inventory methodologies (Lagerwerf et al., 2019). It was assumed that fossil 
energy was replaced by renewable energy sources by 2050, causing no $\mathrm{CO}_{2}$ emissions to keep our assumptions in-line with the EAT-Lancet study (Willett et al., 2019). The only contribution to land use from livestock was the grassland used as feed for ruminants. Other livestock systems were considered landless or had a very small, negligible land use as livestock are fed exclusively LCB.

GHG emission calculations were performed using a food systems approach. A food systems approach assesses emissions from the total diet as opposed to emissions per individual products in a life cycle assessment. Emissions were limited to on-farm, including manure management, enteric fermentation and grassland production. No other animal feed emissions were considered due to the food-based allocation method (Van Hal et al., 2019b).

GHG emissions from terrestrial animals (dairy, beef, pig, broiler and layer) included $\mathrm{CH}_{4}$ and $\mathrm{N}_{2} \mathrm{O}$ from manure management. Methane emissions from manure management were calculated by multiplying volatile solid excretion by the methane conversion factor (i.e. the conversion factor for each manure management system), Bo (i.e. the maximum methane producing capacity for manure) and 0.67 (i.e. the conversion of methane from $\mathrm{m}^{3}$ to $\mathrm{kg} \mathrm{CH}_{4}$ ) (IPCC, 2019a). Volatile solid excretion was calculated using digestibility of protein and organic matter of feed consumed by the animal species (Zom and Groenestein, 2015). Nitrous oxide emissions from manure management included direct and in-direct emissions (the latter resulting from the volatilisation of ammonia and nitrogen (di)oxide) from nitrogen excretion in housing systems (IPCC, 2019a). Nitrogen excretion was calculated by subtracting nitrogen retained in meat/milk/eggs from nitrogen intake (Lagerwerf et al., 2019).

In addition, ruminant systems included $\mathrm{CH}_{4}$ from enteric fermentation and $\mathrm{N}_{2} \mathrm{O}$ from grassland fertilisation. Methane emissions from enteric fermentation was calculated by multiplying gross energy intake by $\mathrm{Y}_{\mathrm{m}}$ (i.e. percentage of gross energy in feed converted to $\mathrm{CH}_{4}$ ) and dividing by 55.65 (i.e. the gross energy content of methane) (IPCC, 2019a). Nitrous oxide emissions from grassland included direct and in-direct emissions (the latter resulting from the volatilisation of ammonia and nitrogen (di)oxide and the leaching of nitrate) from nitrogen fertilisation and manure excretion while grazing (IPCC, 2006b). Grassland fertilisation rates were estimated by the Miterra-Europe model based on the assumption that all organic fertiliser produced by grazing animals is applied to fodder crops (e.g. grassland, fodder maize) in the same region and based on FAOSTAT data on nitrogen mineral fertilizer (Velthof et al., 2009).

GHG emissions from aquatic animals (e.g. high-tropic and low-tropic aquaculture) included $\mathrm{N}_{2} \mathrm{O}$ emissions from the aquaculture system. Nitrogen in un-consumed feed and excreta (nitrogen intake minus nitrogen retained in body tissue) was multiplied by $1.8 \%$ and converted from nitrogen to $\mathrm{N}_{2} \mathrm{O}$ (MacLeod et al., 2019).

GHG emissions were summed into carbon dioxide equivalents $\left(\mathrm{CO}_{2} \mathrm{e}\right.$; 100-year time horizon, 28 for biogenic $\mathrm{CH}_{4}$ and 265 for $\mathrm{N}_{2} \mathrm{O}$; (Stocker, 2014)), and summed with plant-sourced food emissions (see next section) to calculate total GHG emissions. Results were given in GHG emissions per diet per capita per year. 


\section{0 | Chapter 6}

\subsection{GHG emissions and land use from plant-source food}

For plant-source food, average national crop yields and nitrogen inputs per hectare were estimated using the Miterra-Europe model (Velthof et al., 2009) with 2017 as a reference year. From national crop yields and nitrogen inputs, direct and indirect $\mathrm{N}_{2} \mathrm{O}$ emissions were calculated using an IPCC tier 2 approach (IPCC, 2006b). A food-based allocation method was applied where all GHG emissions were allocated to the main food product (e.g. wheat flour) (Van Hal et al., 2019b). To calculate GHG emission intensities and crop yields at an EU level, a weighted average was applied based on harvested area in each country. Not all plant-source food was included in the MiterraEurope model (Lentils, groundnuts, tree nuts, and bananas). The GHG emission intensities and crop yields per hectare were then estimated using global data, reference year circa 2000 (Carlson et al., 2017). Processing of crop into edible food products and food waste along the supply chain (see Section 5.1) were then considered to calculate GHG emission intensities and land use per kg of plantsourced food consumed. 
Chapter 7

General discussion 


\section{2 | Chapter 7}

\section{Introduction}

The adverse impacts of current food systems make transformations towards more sustainable food consumption and production practices a must. Animal-source foods (ASF) play a key role in these transformations, due to their nutritional contributions on the one hand, and their substantial impacts on multiple dimensions of sustainability on the other hand. The objective of this thesis was to investigate the role of ASF in current and potential future food systems, with special emphasis on integrating different sustainability impacts.

This chapter starts with a discussion on the different aspects of sustainability impact assessments of diets and food systems (Section 2). There, I specifically address the three knowledge gaps on differences in ASF solutions, linking sociodemographic and lifestyle factors with sustainability impacts via food choices, and sustainability performance of different consumer strategies. Second, I discuss the compatibility of ASF recommendations in dietary guidelines with circularity principles, thereby addressing the fourth knowledge gap (Section 3). Third, I proceed by discussing suitable substitution options for the proposed reductions of ASF (Section 4). Fourth, I discuss the potential of a consumer-oriented science communication approach that could contribute to facilitate a transformation towards the identified more sustainable food choices (Section 5). Fifth, I discuss consequences of the different scopes employed in this thesis' assessments (Section 6). Finally, in Section 7 I present my conclusions and recommendations.

\section{Sustainability assessments of food systems}

\section{I Sustainability dimensions of food systems}

The identified knowledge gaps required a broad coverage of sustainability aspects, including the environmental, social, and economic dimension. Moreover, the changes addressed with regard to circularity principles required a systemic, and not single-product-focused, context of the impact assessment. To this end, we conducted holistic impact assessments that included several sustainability dimensions, and captured systemic consequences where needed.

We covered the environmental dimension by greenhouse gas (GHG) emissions, land use (Chapters $3,4,5$, and 6), and nitrogen surplus (Chapter 4). Further, by considering circularity principles in Chapters 4, 5, and 6, resource suitability was addressed, which contributes likewise to the environmental pillar of sustainability. In Chapter 2, we showed that results regarding optimal solutions differed between land use and GHG emissions. There, we found that based on currently available estimates, cropland use is lowest when some ASF based on LCB is included in the diet, while GHG emissions are lowest with no ASF. However, in order to verify these results, further research that considers the full systemic consequences of limiting ASF to LCB from a systems perspective is needed.

Moreover, I am aware that the environmental dimension encompasses much more than GHG emissions, land use, and nitrogen surplus. The planetary boundaries concept nicely illustrates nine environmental areas where human actions have substantial impact on (Steffen et al., 2015). Campbell et al. (2017) further quantified the contribution of agriculture to the current state of the planetary 
boundaries, whereas Willett et al. (2019) defined the share of the planetary boundaries that would be available for food systems. Next to land-system and climate change, agriculture substantially contributes to altering the biogeochemical flows of nitrogen and phosphorus, the biosphere integrity, and freshwater use. Currently, many studies focus mainly on GHG emissions, land use, and water use aspects for environmental impact assessments of dietary scenarios (Ridoutt et al., 2017). Including additional areas of the environmental dimension as well as contributing to improving measures to represent these is an important area for future research.

Although not all aspects of the environmental dimension are equally well represented in impact assessments, other dimensions - such as the social and economic dimension - are even less frequently included (Béné et al., 2019). The social dimension of sustainable food systems encompasses social welfare and related impacts during production, processing, transport, and retailing, and can also include access to healthy and safe food of consumers (Garnett and Godfray, 2012). Sometimes, it is moreover referred to in combination with ethical, cultural, and equity aspects (Hebinck et al., 2020). Based on our definition of sustainability, we included both social risks and human health aspects in the social dimension. To represent social risks, we employed the Social Hotspots Index based on the Social Hotspots Database in Chapters 3 and 4 (Benoit-Norris et al., 2012; Norris et al., 2014). Since this index is calculated based on a combination of single indicators covering topics from labour rights, health and safety, to governance and community infrastructure, we managed to cover a broad range of social aspects up until farm gate (Benoit-Norris et al., 2012). While the Social Hotspots Database provides an important base for future research, further development of it and wider applications are needed for a suitable representation of the social dimension (Huarachi et al., 2020). Moreover, other social issues, such as animal welfare, are not considered by this database. To cover the social dimension beyond a human-centric standpoint, the Social Hotspots Database would thus need to be complemented by additional impact assessments.

To represent the human health aspect of the social dimension, we employed the Alternate Healthy Eating Index (AHEI) (Chapters 3 and 4). Impacts of food choices on human health are inherently hard to predict, since human health outcomes depend, among others, also substantially on lifestyle aspects, such as physical activity and smoking (Conlon and Bird, 2015; Feigin et al., 2016). Nevertheless, human health aspects are increasingly considered in sustainability assessments of food systems. The measure employed here, i.e. the AHEI, is an index that links food choices to potential diet quality. In scientific literature, next to the AHEI also other measures, such as the NRF9.3 (Walker et al., 2018) or the relative disease risk (Tilman and Clark, 2014) are employed. However, although (part of) these measures are based on associations of food choices with occurrences of non-communicable diseases in large cohort studies, good performance on these indicators does not necessarily mean that a balanced and favourable diet is obtained. For example, the AHEI is composed by 11 subgroups that are aggregated to a single score, and thereby faces known issues with aggregation and reference values of indices (De Olde et al., 2016). To ensure a balanced diet beyond indices, clear substitutes could be defined that result in comparable nutritional outcomes, or ranges e.g. based on food-based dietary guidelines (FBDGs) could be employed.

With regard to the economic dimension of sustainable food systems, there is currently little consensus on how to include it into assessments (Béné et al., 2019). In our assessments in Chapters 


\section{4 | Chapter 7}

3 and 4, we included the economic dimension by dietary costs. While this indicator can give a basic understanding of the changes in cost of diets, it has several drawbacks. First, we did not differentiate between in-house and out-house consumption, which would obviously affect dietary cost. Second, while current prices are a valid indicator for current diets (Chapter 3), they need to be interpreted with more caution when dietary changes were to be implemented at population level. Then, the changes assumed would not only alter the demand of different food products, but also the structure of the agricultural sector, with consequences for price levels. With this, relative prices would change, and dietary costs could end up being substantially different from estimates with current price levels. Third, current costs do not encompass externalities. In order to include these effects, for example a true cost approach would be required. Since capturing these effects would require a sophisticated representation of the economic aspects of such transformations and thus different modelling tools, including current prices can nevertheless give a rough estimation within the modelling tools applied here, but need to be interpreted with care.

In conclusion, solutions for more sustainable food systems clearly depend on the dimensions included, and on the indicators chosen to represent these dimensions. It is, therefore, crucial to set results into the context of the chosen indicators, and discuss the related assumptions, foci, and omissions critically.

\subsection{Methodological choices and differences in dietary solutions}

Our results showed that part of the differences of the ASF reduction solutions can be linked to methodological choices in the impact assessments (Chapter 2). On the one hand, we identified studies that mainly employ fixed impact intensities and consumption-oriented scenario specification. Examples for these scenario specifications are dietary guidelines, vegetarian scenarios, or the reduction of all red and processed meat. These studies mostly recommended to reduce beef and pork, while chicken, dairy, eggs, and fish can be decreased to a lower extend - or, in some cases, even increased and taken as substitute. On the other hand, we identified studies that consider systemic consequences in their assessments and mainly specify scenarios from a resource-use perspective. In these studies, the dietary solutions identified included a larger reduction of chicken and pork. In these latter approaches, scenarios were generally specified from a production-oriented perspective. In other words, in these scenarios, specific attributes of ASF production were considered to formulate scenarios that consider suitability of resources for agricultural production. In addition, these studies considered product packages between multiple outputs from the processing of single commodities in their scenario definition. For ASF, this is particularly relevant for the relation milk - meat in dairy production systems, and eggs - meat in egg production systems likewise. As an example, employing a vegetarian scenario at the level of the whole population would result in inconsistencies, since the associated meat of the milk and eggs production is not considered in the scenario formulation.

Recently, Karlsson et al. (2020) demonstrated that when the European Union would halt soybean feed imports, ruminants would be favoured over poultry and pigs. This example illustrates what the latter methodological approaches can capture, and what the former cannot. Impact assessment 
approaches that do not consider interlinkages between different commodities cannot capture feedfood competition, which should, in the interpretation of the results, be noted.

As indicated by the respective orientation of the scenarios, these two main solution spaces differ in their priority given to human health aspects (consumption-oriented scenarios) and resource use aspects (resource-oriented scenarios). Notably, both solution spaces aim to provide more environmentally-friendly solutions, yet the different methodological choices in their environmental impact assessments cause differences in results. It is, therefore, crucial to accompany results of impact assessments of dietary scenarios with clear notions on which aspects are considered, and which priorities result from this. With this, I addressed the first knowledge gap on causes for differences in dietary solutions for ASF among studies.

\subsection{Sociodemographic and lifestyle factors of current diets}

In order to know how to better target dietary improvements such as reductions of ASF, more knowledge on current diets is needed. In Chapter 3, we showed that different sociodemographic and lifestyle factors revealed different degrees of association with specific food choices. The sociodemographic and lifestyle factors included encompassed sex, nationality, language region, age group, education, civil status, income group, smoking status, Body Mass Index group, and being currently on a weight-loss diet. While nationalities, language regions, age groups, and smoking status seemed particularly distinctive, income or educational groups seemed hardly relevant. Food choices and the resulting sustainability impacts thus differed markedly between these distinctive factors, suggesting that these can (at least partly) be relevant to, for example, target nutrition campaigns. The two factors nationality and language region point at cultural differences that cause the differing levels. While the former, nationality, relates to cultural factors that drives these differences, Switzerland is a special case with respect to the differences in language regions (Pestoni et al., 2019). Due to the clear geographical distinction of these language regions, these pose an easyto-implement differentiation for potential nutrition campaigns.

Resulting from these identified differences, measures that target different sustainability groups will potentially affect distinct population groups differently. Therefore, careful design of measures is needed in order not to structurally disadvantage specific population groups. Income poses an interesting example, where we found no statistically significant differences, indicating that measures to improve specific impacts would not structurally disadvantage lower income groups. Supporting this, Keats and Wiggins (2019) noted that as income becomes less determining for diets, while other factors become relatively more important over time, public policy can have increased influence on future diets.

To sum up, we successfully identified correlations between sociodemographic and lifestyle factors, food choices, and sustainability performance, thereby addressing the second knowledge gap on linking sociodemographic and lifestyle factors with sustainability impacts of food choices. These insights can provide entry points for targeted nutrition campaigns. However, unravelling the underlying causes for the respective differences requires further research. In fact, factors influencing dietary behaviour, such as emotional factors, food environment, education, and culture, need to be 


\section{6 | Chapter 7}

carefully studied in order to understand these potential causal mechanisms (Beydoun, 2014; Kubik et al., 2003; Shepherd, 2005).

\subsection{Synergies and trade-offs of consumer strategies}

The different consumption- and production-side strategies assessed in Chapter 4 revealed multiple synergies and trade-offs between strategies and between impact categories. On the consumption side, we assessed two dietary changes (following a vegetarian diet and adhering to the Swiss FBDG). On the production side - but also framed as a consumer strategy, thus by selecting the food products accordingly - we assessed increased consumption of organically produced foods (also in combination with circularity principles), as well as increased consumption of domestic produce. Moreover, reducing food waste at consumption stage was introduced as an additional strategy.

The major trade-off between strategies related to the order of magnitude of the achievable sustainability improvement (Chapter 4). The dietary change strategies as well as the increased consumption of organically produced foods revealed potentially strong sustainability improvements. Reducing food waste at consumption stage showed lower potential for improvement compared to the other strategies assessed, which is in line with previous findings (Springmann et al., 2018a). However, food waste reduction improved all impact categories simultaneously, while all other strategies - except the vegetarian diet at lowest implementation level - caused deterioration on other impact categories.

Moreover, we identified synergies and trade-offs between impact categories both for the consumption-side strategies as well as for the production-side strategies (Chapter 4). For the consumption-side strategies, Figure 1 displays the main synergies and trade-offs identified. Notably, all these identified synergies and trade-offs depend on the starting level and the order of magnitude of change. For example, when starting at low levels of pulses and assessing a slight increase, this will most likely not affect social risks. The synergies and trade-offs identified in Figure 1 need, therefore, to be interpreted in context of the results of Chapter 4, and cannot directly be generalised. I will first discuss the identified synergies, and then proceed with the trade-offs.

The main synergies identified occurred between the environmental, economic, and human health aspects of the food groups pulses, fruits, vegetables, and certain types of meat. The studied increase in pulses (Chapter 4) led to synergies between environmental impacts, human health outcomes, and dietary costs. On the environmental dimension, this improvement could be traced to lower impact intensities of pulses as compared to the different types of meat, which pulses were substituting. Further, for low starting levels of pulses, an increase led to an increased score of the AHEI. Moreover, since pulses are generally relatively cheap, the dietary costs could be reduced, thus leading to synergies with the economic dimension as well. For fruits and vegetables, this latter effect could not be found, since these food groups are generally costlier. Next to this, however, the same patterns arose as for pulses with regard to the environmental and the human health dimension. 
Increase pulses

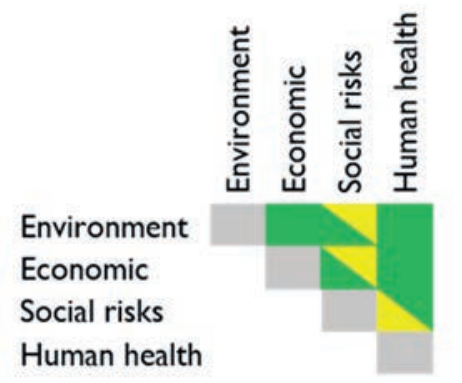

Decrease beef and pork

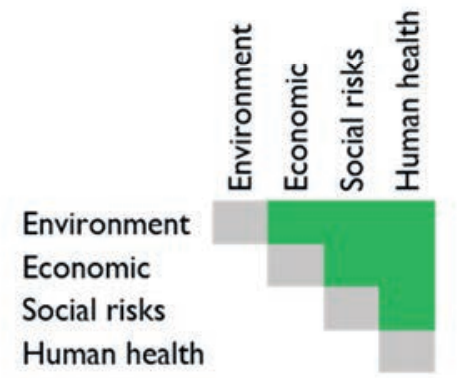

Increase fruits and vegetables

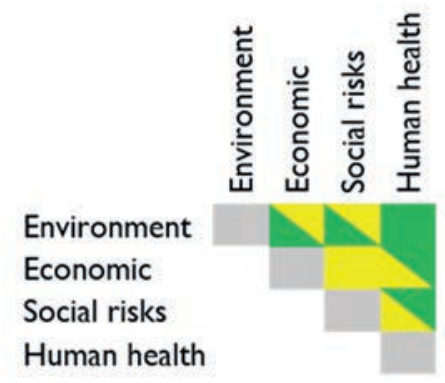

Decrease chicken and fish

\section{Environment \\ Economic \\ Social \\ Human health}

Figure I. Synergies and trade-offs of increases and decreases of selected food groups between different sustainability dimensions. Green: improvement. Yellow: detriment. Environment: greenhouse gas emissions, land use, nitrogen surplus. Economic: dietary costs. Social risks: Social Hotspots Index. Human health: Alternate Healthy Eating Index.

With regard to different types of meat, different classification schemes occurred for the different dimensions. While we were able to distinguish between the animal production systems that lie behind the meat for the environmental, social, and economic dimension - resulting into the classification of chicken, pork, and beef - the AHEI required a distinction of red and processed meat. Hence, we classified pork and beef as red meats (while in Chapter 3, we also distinguished processed meat). Based on this classification, the identified synergies occurred between the environmental and the economic dimension for a reduction of all types of meat. This was driven by higher environmental impact intensities as well as higher prices for the types of meat included. Furthermore, for the human health dimension, reduction of red (and processed) meat led to an improved score of the AHEI, thus also resulting in synergies.

The main trade-offs arose for fruits and vegetables between the environmental, economic, and social, as well as between the economic, social, and human health dimensions (Figure 1). In addition to that, trade-offs occurred for pulses between the social and all other dimensions. Furthermore, from Chapter 3 we can conclude that for chicken and for fish, trade-offs emerged between the environmental and economic dimension and human health.

The trade-offs for an increase in fruits and vegetables occurred due to benefits for the human health dimension and the comparatively better environmental performance on the one hand, and higher 
prices and potential to increase social risks on the other hand. For an increase in pulses, effects were similar, except that lower prices for pulses inverted the effects for the economic dimension. The trade-offs for a reduction of chicken and fish were caused by their potential benefits for the human health dimension, in combination with adverse impacts on the environmental and economic dimension (the latter being higher prices; Chapter 3). More concretely, for white meat, i.e. chicken in our case, as well as fish, a certain amount can contribute to good performance on some sub-scores of the AHEI, mainly via their poly-unsaturated fatty acids content.

For the production-side strategies, clear trade-offs emerged for increasing the share of organic produce (Chapter 4). This change revealed known effects of large-scale conversions to organic agriculture, such as the increased land needed to reach similar production levels as under conventional practices (Seufert et al., 2012). This result is a direct consequence of lower yields in organic agriculture, which are caused by the restrictions (e.g. no artificial fertilizer, no synthetic pesticides) of this production standard (De Ponti et al., 2012; Ponisio et al., 2015; Seufert, 2019; Seufert et al., 2012). It has been shown, however, that with strategies such as intercropping, this yield gap could be reduced (Ponisio et al., 2015). Moreover, the lower production intensity of organic agriculture was found to have positive effects on for example soil health as well as biodiversity on fields (Lori et al., 2017; Mäder et al., 2002). However, with our measure of land use, we were not able to capture these effects. Further, nitrogen surplus showed a substantial decrease under organic cultivation, which is a positive consequence of this cultivation standard. In a second production side strategy in Chapter 4, we enhanced organic production practices with circularity principles targeting at avoiding feeding animals with food, or producing feed on areas suitable for food production. With this additional change, all environmental impact indicators revealed a substantial improvement. For the circularity principles, the scope of the assessment is crucial: since we employed consumer strategies at the level of individuals in Chapter 4, we were able to assess this strategy without linking it to dietary changes. However, at the population level of food systems, such a strategy would only be feasible in combination with reduced animal numbers and consequently reduced consumption of ASF. This latter aspect will be further discussed in Section 3.

Next to these synergies and trade-offs, we identified an additional trade-off between the human health dimension and implementing circularity principles (Chapter 6). This effect was caused by byproducts that arise from processing and was, more concretely, identified for processing by-products of wheat milling. In fact, while eating whole grains is mostly associated with beneficial effects on human health (Chiuve et al., 2012), this in turn diminishes the amount of available by-products as animal feed, which would occur from refined grains. However, since use for human food has highest priority according to recently proposed circularity frameworks (De Boer and Van Ittersum, 2018; Muscat et al., 2019), this does in fact not need to be seen as a trade-off, but rather as an effect that needs to be considered in designing such farming and food systems.

In conclusion, the identified synergies and trade-offs between strategies and between impact categories provide insight into the sustainability performance of different consumer strategies, and thus address the third knowledge gap of this thesis. While incremental improvements can be achieved across all dimensions assessed, profound improvements revealed trade-offs - unless the strategies are combined in a synergistic way. Consequently, the need to integrate promising 
consumption side strategies, such as adhering to dietary guidelines, consistently with promising systematic changes, i.e. circularity principles, emerged, which is discussed in the next section.

\section{Dietary guidelines and circularity principles}

We investigated the compatibility of ASF recommendations and circularity principles at two geographical scopes: at national levels (Chapter 5) and at European Union EU-28 level (Chapter 6). For both assessments, we modelled systemic changes at population level. In Chapter 5, we employed five national dietary guidelines (Bulgaria, Malta, Netherlands, Sweden, and Switzerland), whereas in Chapter 6, we investigated the compatibility with the dietary recommendations of the EAT-Lancet commission. While for the latter, ASF protein of the recommendations could be met (although not the exact composition), we found this not to be feasible for the national dietary guidelines assessed. Nevertheless, considering circularity aspects in dietary guidelines by linking the types of ASF to available low-opportunity-cost biomass (LCB) offers large potential to improve resource use efficiency in food systems, and improving environmental performance. Notably, when assessing systemic changes at population level, the impact assessment has to be able to capture these systemic consequences. Therefore, we employed modelling approaches (resource allocation model (Chapter 5 and 6) and SOLm (Chapter 5)), that consider these altered animal feed compositions in the impact assessment.

As a contribution to a balanced diet, ASF can fulfil different nutritional functions. Thus, depending on the plant-source food part of the diet and the resulting nutritional contents, ASF can be selected specifically to close the remaining nutritional requirements. In Chapter 5, we showed the selected types of ASF when protein and three alternative groups of nutrients were maximised based on available LCB. For the maximisation of protein, dairy and the associated meat showed a substantial increase, while for the maximisation of minerals (calcium, iron, and zinc) eggs showed a stronger increase. The group of vitamins (vitamins $A$ and $B_{12}$ ) to be maximised resulted in similar results as the protein maximisation scenario. Differences could be found when the sum of the omega- 3 fatty acids $\alpha$-linolenic acid (ALA), docosahexaenoic acid (DHA), and eicosapentaenoic acid (EPA) were maximised. Then, fatty fish became relatively more important.

I am, however, aware that focusing on single nutrients does not necessarily ensure a balanced diet, since the resulting nutritional content of the sum of all foods consumed, their effective uptake in the human body, and associated human health outcomes differ due to many factors (Conlon and Bird, 2015; Feigin et al., 2016). These factors are for example the interlinkages - and the amplification or alleviation, depending on the combination - of intake of different foods and nutrients (Anitha et al., 2020). A second example is the respective gut microbiome: depending on the composition of the microbiome, nutrients can be taken up to different extents by the human body (Asnicar et al., 2021; Senghor et al., 2018). Although these factors suggest that single nutrients need to be interpreted carefully, our results from Chapter 5 give an overview of the potential nutritional functions of ASF in a balanced diet.

When considering current overconsumption in many high-income countries, and the fact that four out of the five national FBDGs assessed recommend food quantities that lead to substantially more 
protein than the daily requirements of about 50-60 g per capita (EFSA, 2017; WHO, 2007) (Chapter 5), we can conclude that a part of ASF consumed as well as recommended can be reduced without substitutes. Regarding the recommendations, it can be argued that some parts of the population need more protein than the average daily requirements (e.g. during pregnancy, elderly, or heavy physical work), which is an explanation for these relatively high implicit protein contents. An additional explanation is that in order to make dietary guidelines better implementable, current dietary habits play a stronger role for the definition, whereby current high protein intake is translated to guidelines. To address this, reducing implicit protein content from ASF for general recommendations and complementing these with specific recommendations for population groups that have higher protein requirements could be a suitable and more transparent solution.

To investigate the compatibility of ASF recommendations in the EAT-Lancet diet and circularity principles, we assessed different restrictions on the composition of the ASF (Chapter 6). When the ASF composition was not restricted, the original protein contents could be met - but the composition then differed substantially from the original one, containing mainly dairy, beef from dairy systems, and pork. In contrast, when the composition was restricted to the ratio of the composition in the EAT-Lancet diet, the original protein contents could not be met. In the composition of the original EAT-Lancet diet, larger quantities of chicken meat over beef and pork are recommended. The case of chicken thus presents a trade-off between nutritional considerations on the one hand, and ability to convert LCB on the other hand. This underlines the need to clarify the nutritional function of the ASF recommended.

Notably, the quantities of ASF that can be produced based on LCB largely depend on LCB availability, and hence also the potential compatibility with ASF from dietary guidelines. In Chapter 6 , we for example varied the degree of processing of wheat products. This showed that consumption of refined grains results in more available by-products from this processing stage and thus more available LCB, whereas this LCB stream diminishes with consumption of wholegrains. Moreover, grassland availability largely drove the resulting ASF quantities of dairy and the associated meats and fats, which in multiple cases contributed most to total ASF protein from LCB (Chapters 5 and 6). However, the extent to which grass resources should be counted as LCB is debatable. Part of grass resources are sourced from marginal areas or from temporary grassland with an agronomic function in crop rotations. Grass resources that exceed this threshold come, however, with opportunity costs, and therefore need to be critically evaluated (Van Hal, 2020).

In conclusion, we found that neither the five national dietary guidelines nor the EAT-Lancet diet were compatible with ASF limited to LCB. For the EAT-Lancet diet, this was mainly due to differences in composition, whereas protein contents of the ASF recommendations in the five national dietary guidelines could not be reached regardless of the composition. We moreover showed that if ASF recommendations would be made compatible with limiting feed to LCB, the environmental performance and resource use efficiency could be substantially improved. With this, I answer the fourth knowledge gap identified in this thesis. 


\section{Suitable substitution options}

In our assessments, we mainly focused on the required degree of reduction of ASF, suitable composition of the remaining ASF, and potential ways of producing this remaining ASF. However, reductions of ASF that go beyond nutritional requirements need to be substituted with suitable plant-based protein sources. In this section, I will therefore set assumptions from our assessments in context with findings from literature on suitable substitution options.

In the first consumer strategy in Chapter 4, we assumed that the reduced meat consumption is substituted with pulses. Although pulses do constitute a promising substitution option for ASF, several factors need to be taken into consideration for this option. First, protein quality and nutrient availability in general can be lower than in most ASF (Nosworthy et al., 2017). To compensate for this, pulses need to be complemented with a variety of other plant-source foods, such as grains or vegetables (Anitha et al., 2020). Thereby, in sum all required amino acids can be sourced from these alternatives (Mariotti and Gardner, 2019). Moreover, practices such as fermentation or biofortification can also improve the bioavailability of plant-based foods (Proulx and Reddy, 2007; Talsma et al., 2017). Second, consumer acceptance for increased consumption of pulses can be low, which makes the implementation of this substitute challenging. Szczebyło et al. (2020) found that a potential cause for this is the lack in knowledge on how to prepare pulses best. The absence of this knowledge can lead to unintended effects, such as flatulence - and hinder widespread use of pulses as a substitute. In addition, taste and consistency cannot be directly compared to ASF. Additional promising alternatives are algae and insects - the latter though not plant-based - to be used in processed foods (Parodi et al., 2018).

Other substitution options are plant-based alternatives that aim to imitate the respective ASF. For these, the required behaviour change is substantially lower, and hence also potentially the consumer acceptance (Michel et al., 2021). For milk and milk products, many plant-based alternatives exist. These however mostly do not meet the nutritional quality, especially in terms of protein, of the original dairy option (Chalupa-Krebzdak et al., 2018). Other examples are meat imitations and cultured meats. Remaining challenges for the former are consistency and taste in comparison to real meat (Michel et al., 2021), and for the latter, market maturity and price as well as environmental impacts (Post et al., 2020; Tuomisto, 2019). Additional concerns arise from the degree of processing and additives required: most of these alternatives are highly processed and require stabilisers and emulsifiers, thereby causing concerns for human health, and additional environmental pressures from this processing stage (Van der Weele et al., 2019).

In sum, alternatives for the substitution of ASF exist in abundance, in varying degrees of similarity to ASF. These alternative food choices require attention as to what is consumed and in which combination - with diversity being key. With this, they can form an important contribution to a mainly plant-based and healthy human diet, and are in line with the recent planetary health diet proposed by Willett et al. (2019). 


\section{Consumer-oriented science communication}

Much scientific literature, to which our studies contribute, presents solutions for more sustainable diets from more sustainable food systems. At the same time, the need for suitable science communication approaches becomes apparent (Dahlstrom, 2014). Due to the complexity and multidimensionality of many scenario studies, these often cannot be directly employed for science communication with consumers and other stakeholders. Yang and Hobbs (2020) have shown that employing narratives can be an effective tool to communicate scientific results effectively. As a basis for this, we presented the results in Chapter 4 as consumer strategies. In a follow-up dissemination project, these consumer strategies were enhanced with narratives, resulting in six consumer profiles. These were then processed into two different web-based communication approaches: first, an approach that included results of Chapter 4, and enhanced the consumers with characteristics, such as name and motivation (SNF, 2020). The second approach dived further into the narrative approach by enhancing the characters, i.e. the consumers, with comics (SGE, 2020). These comics presented the motivation and plot that led the respective consumer to its behaviour change.

With the results of Chapter 4 and the follow-up web-based applications, we propose a science communication approach that is based on consumer narratives. However, in order to test whether the proposed approaches can be an effective tool for such communication, further research is necessary. Such research could complement these proposed approaches with alternative approaches, and engage in consumer dialogue in order to test potential behavioural changes that can potentially be triggered by these approaches. This could yield insight into requirements of such science communication approaches, which could in turn be a complementary part of future research engaged in sustainability assessments towards more sustainable food systems.

\section{Consequences of scopes}

The different scopes employed in this thesis shape the resulting requirements for change towards more sustainable food systems, and the potential role of ASF therein. We started with reviewing literature on dietary solutions that gave insight into methodological choices that drive differences between these solutions, including a variety of scopes. Then, we assessed multiple sustainability impacts of current diets (present; individual) for Switzerland (national). This assessment revealed which sociodemographic and lifestyle factors can be linked to which sustainability performances, thereby giving insights into potential entry points for targeted actions to improve the sustainability performance of Swiss dietary choices. Further, we assessed multiple sustainability impacts of consumer strategies (future; individual) for Switzerland (national). Due to the focus on individual consumers, no systemic changes were required. However, we found that by combining different strategies, large improvement potentials on all sustainability dimensions could be unlocked - which would, in turn, require systemic changes.

Consequently, we combined these strategies to assess systemic changes, which we implemented by modelling adherence to dietary guidelines in combination with circularity principles for different contexts (future; population; national and European Union EU-28). To reach the changes assumed 
in these latter studies, a systemic transformation of food systems is needed. While consistently implementing this across a larger geographical scope would require (supra-) national coordination, we also showed that the concrete implementation is highly context-specific, depending on cultural and geographical circumstances. To sum up, depending on the envisioned degree of change as well as the priorities on the different dimensions of sustainability, suitable combinations of coordinated action at (supra-) national and thus population level, and implementation of consumer strategies at individual level, are required.

\section{Conclusions and recommendations}

The current environmental, social, and economic challenges of food systems necessitate actions towards more sustainable consumption patterns and production approaches. This thesis investigated strategies towards more sustainable food systems, with special emphasis on the role of animal-source food (ASF). To this end, different geographical, temporal, and demographic scopes were employed. Thereby, both current and potential future food systems were assessed, as well as improvement strategies at individual level up to systemic changes at population level. Based on the presented results, I draw four main conclusions with implications for future scientific work, policy makers, and society in general.

First, although we revealed and confirmed many synergies between the different sustainability dimensions for the improvement strategies assessed, also trade-offs occurred. Trade-offs were particularly pronounced for social risks, where increases in plant-source foods, such as vegetables, fruits, and pulses, increased social risks - thus potentially leading to unwanted effects, while increases in these food groups improved the other sustainability dimensions. This is thus an area that should be further investigated, and complemented with case studies that study the on-site effects of such increased demands. Instead of avoiding the consumption of these food products, policy makers as well as society in general should ensure that increased consumption of these is complemented with efforts to improve social aspects, such as working conditions, in the respective countries of origin.

Second, we showed that methodological choices drive differences in environmentally-friendly dietary solutions, in particular for ASF. While studies that use fixed impact assessments and consumption-oriented scenarios tend to suggest that beef should be reduced most, followed by pork and chicken, studies that consider systemic consequences in the assessment, including co-product links and restrictions on resource use and sink capacities, tend to recommend larger reductions for chicken and pork. Notably, in the former approaches, the role of ASF is restricted to current production systems, while in the latter, ASF is mostly limited to available low-opportunity-cost biomass (LCB). By this limit, feed-food competition is avoided and circularity principles can be considered. Making these underlying assumptions explicit in future scientific work is key for improved transparency regarding dietary solutions, thus enabling effective communication and policy design. In particular, it has to be emphasised that current environmental impact intensities cannot capture the environmental consequences of large-scale food system changes. 
Third, the ASF quantities and compositions in the national dietary guidelines assessed as well as in the EAT-Lancet healthy reference diet are incompatible with ASF limited to LCB. Different ASF, and especially chicken, revealed trade-offs between nutritional considerations and the ability to convert LCB. Limiting ASF to LCB while considering circularity principles would yield substantial environmental benefits in terms of reduced greenhouse gas emissions and reduced resource use. To be able to exploit these benefits, the role of ASF limited to available LCB in dietary guidelines as well as in current diets would need to be further investigated. Moreover, the nutritional function as well as the permissible substitutability of ASF in dietary guidelines should be made more transparent, such that ASF can be better targeted to cover specific nutritional requirements.

Fourth, we assessed strategies both at individual and at population level, including incremental and systemic changes likewise, which resulted in differentiated implications for effective transitions towards more sustainable food systems. On the one hand, strategies at individual level can be achieved by changes in individual consumption behaviour, which can, in sum, contribute to systemic changes. Individual behavioural changes, in turn, need to be facilitated with changes in the food environment. On the other hand, systemically limiting ASF to LCB would require clear policy agendas as well as shifts in production practices and industry solutions, which then need to be complemented with corresponding consumption changes.

In conclusion, the role of ASF in more sustainable food systems can be manifold - and depends largely on the required nutritional contributions as well as on defined priorities for different dimensions of sustainability. Importantly, to thoroughly assess the role and potential of ASF for improved sustainability, adequate methods need to be used and adopting a systems-level analysis going beyond product-specific efficiency measures is unavoidable. 


\section{Appendix A}

A1 Review approach

A2 Studies not matching the typical methodological approaches

A3 Comparing environmental performance between scenarios 


\section{Al Review approach}

The aim of this study is to identify causes for differences in conclusions of studies assessing environmental impacts of diets. The literature review conducted to address this aim followed four stages, cf. (Moher et al., 2009): first, identification of records, second, screening of records, where duplicates were removed and decision for exclusion was based on the title and abstract of the identified records. In the third stage, eligibility of records was assessed based on the full-text article. Finally, in stage four, the final set of articles was defined for inclusion in the subsequent analysis.

Stage 1: Identification. The literature review was performed in the databases Web of Science (ISI) by keyword search and Boolean operators on 25-02-2019. Keywords and Boolean operators were combined to queries as follows:

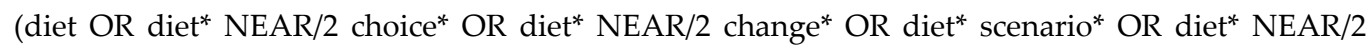
pattern* OR diet* NEAR/2 type*) AND (climate OR greenhouse gas OR land) AND (sustain* OR impact $\left.^{*}\right)$

(diet* NEAR/2 human* OR diet* NEAR/2 choice* OR diet* NEAR/2 change* OR diet* NEAR/2 scenario* OR diet* NEAR/2 pattern* OR diet* NEAR/2 type* OR food NEAR/2 demand OR feed* NEAR/3 world) AND (climate OR greenhouse gas OR GHG OR land) AND (sustaina* OR env* NEAR/2 impact*)

Following the database search, references of previous review articles on related topics (Aleksandrowicz et al., 2016; Jones et al., 2016; Ridoutt et al., 2017; Van Zanten et al., 2018) were screened to identify additional records that were potentially missed. This led to a total of 605 identified studies.

Stage 2: Screening. In this phase, all articles identified in the first stage were screened, based on the information given in the title and abstract. Studies were included if they met all five inclusion criteria:

(i) peer-reviewed English-language publication;

(ii) published between January 2014 and date of search (25-02-2019)

(iii) assessment of environmental impacts - with a minimum requirement of either land use (LU) or greenhouse gas emissions (GHG) of potentially more sustainable diets being covered;

(iv) comparison of environmental impacts of an alternative diet with the current situation or between alternative diets; and

(v) environmental impact assessment for diets on a regional or global scale, focusing on studies within Europe or the US and global assessments. Thus, on the one hand, assessments of diets at the level of individual persons are excluded. And on the other, studies from other parts of the world - such as Africa or Asia - were excluded, to minimise the potential for sources other than methodological choices that influence the recommendations.

In stage 2, a total of 539 studies was excluded. 
Stage 3: Eligibility. Remaining articles were downloaded and their full text assessed, whether it matches all inclusion criteria defined above. Of these, another ten were excluded; 5 due to a geographical scope that was not compliant with our inclusion criteria (Hendrie et al., 2014; Li et al., 2016; Milner et al., 2017; Song et al., 2019), 2 due to the assessment of individual diets (Scarborough et al., 2014; Walker et al., 2018), 1 due to direct assessment of current diets (Hyland et al., 2017), 1 because not complete diets were assessed (Rohmer et al., 2019), and 1 study was not accessible (Sabate and Soret, 2014).

Stage 4: Inclusion. After this procedure, a total of 56 studies remained and was included in the subsequent analysis, cf. Frehner et al. (2019).

\section{A2 Studies not matching the typical methodological approaches}

Not all studies identified in the literature review correspond to one of the identified typical methodological approaches. For scenario specification, 4 studies did not apply the proposed combination of values. The studies (Erb et al., 2016; Springmann et al., 2018a; Willett et al., 2019) follow products in the allocation, but consider restrictions on resource use and sink capacities, thus do correspond to consumption- or resource-oriented scenario specification exactly. One study (Hedenus et al., 2014) on the contrary, follows nutrients after the allocation, but does not account for restrictions on resource use and sink capacities. Further, for environmental impact assessment, three studies follow products after the allocation and consider restrictions on resource use and sink capacities (Springmann et al., 2018a; Willett et al., 2019), and additionally, consequences (Erb et al., 2016). (Saxe, 2014) and (Goldstein et al., 2016) also follow products in the allocation and consider consequences (by using CLCA). One study (Hedenus et al., 2014) follows nutrients in the allocation and considers consequences, but does not consider restrictions on resource use and sink capacities. Lastly, one study (Röös et al., 2016) follows nutrients in the allocation, performs the inventory analysis on product level, and considers consequences and restrictions on resource use and sink capacities. These combinations are not covered by either FIA or SCA, and therefore, these studies do not match the identified typical methodological approaches.

It has to be noted that this lack of congruence is not an issue of quality. The typical methodological approaches in the studies reviewed are highly diverse, which makes it difficult to build categories where all studies fit into.

In Figure A2.1, the recommendations of the studies not falling into one of the typical methodological approaches are presented. We see that also these studies agree on a reduction of most animal-source foods (ASF) categories. With regard to optimal range and rationale for change, the studies not fitting to the typical methodological approaches for scenario specification resemble those from the consumption-oriented scenario specification. For the typical methodological approaches on environmental impact assessment, recommendations of studies are mixed, thus, could be part of both FIA and SCA. 


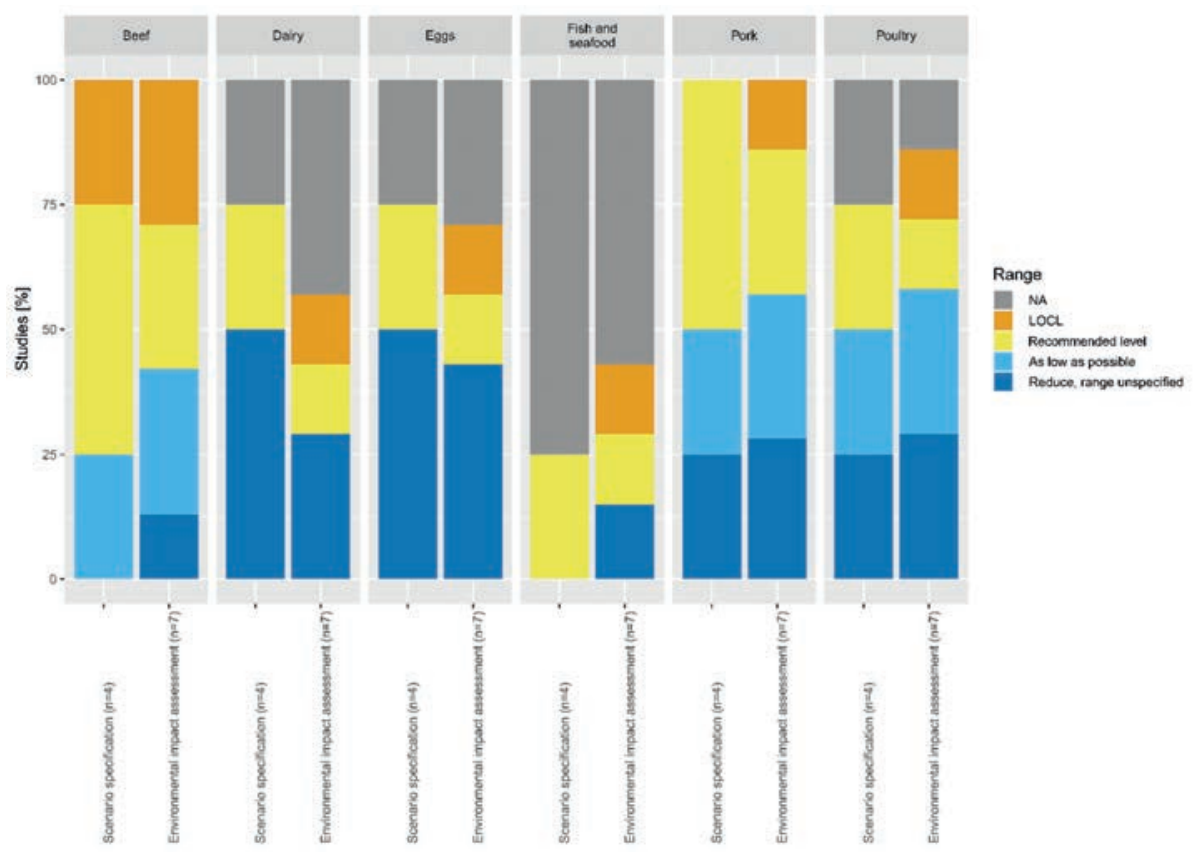

Figure A2.1. Inventory of optimal ranges for animal-source food reduction solutions to achieve reduced environmental impacts of the food system (\% of the studies excluded from scenario specification and environmental impact assessment, respectively). Colors indicate the proposed range; low-opportunity-cost livestock (LOCL), recommended level, as low as possible, reduce with unspecified range, and not assessed (NA).

\section{A3 Comparing environmental performance between scenarios}

Environmental performance between studies and scenarios can be compared relative to a vegan scenario or relative to a reference scenario. Results relative to the vegan scenario, as presented in Figure 3 in the main paper, are an update of Figure 1 in Van Zanten et al. (2018). Only studies that calculated a vegan scenario were included in this Figure, to be able to demonstrate the range for LU and GHG emission results for the whole range of shares of ASF in human diets. When comparing results relative to the reference scenarios (see Figure A3.1), more studies can be included, because most studies include reference scenarios while only some studies include vegan scenarios. Figure A3.1 reveals similar results as can be obtained from comparing impacts relative to vegan scenarios; for GHG emissions, results indicate a clear negative correlation between $g$ of ASF-based protein in the human diet, while for LU, results are more dependent on the methodological approach chosen and land type included. 

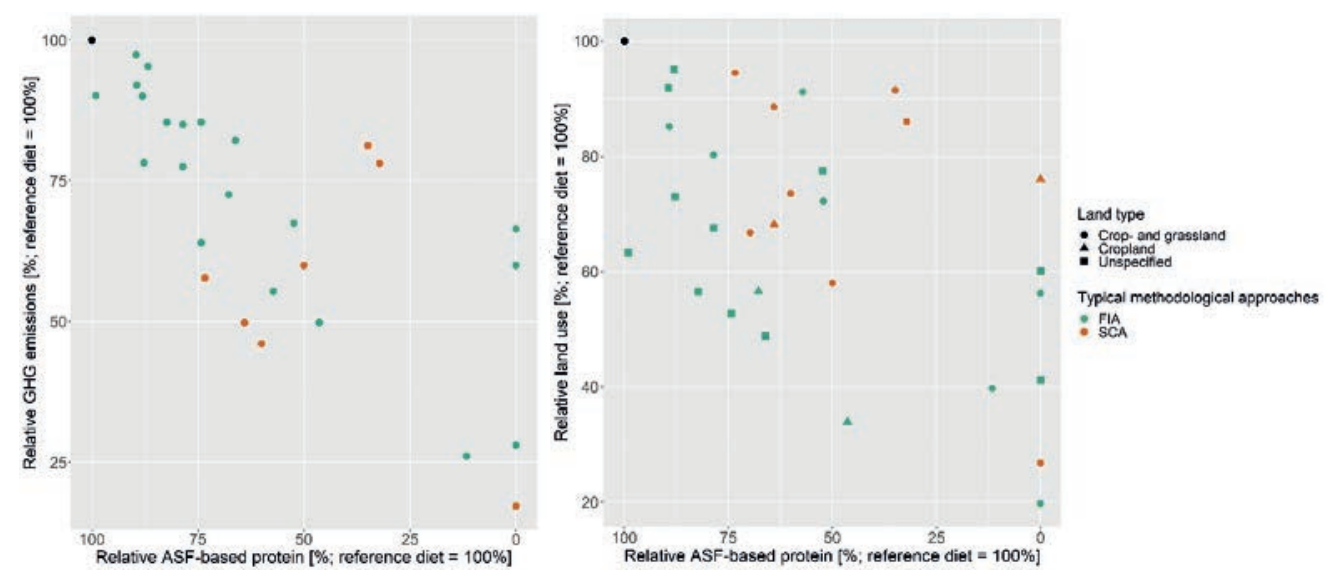

Figure A3. I. Left: relative greenhouse gas (GHG) emissions per relative animal-source food (ASF)-based protein. Right: relative land use per relative ASF-based protein (land type is indicated by shape). Each dot represents one dietary scenario. Colors indicate typical methodological approaches; fixed impact assessment (FIA) and systemic consequences analysis (SCA). ASF, land use, and GHG emissions of dietary scenarios are presented relative to reference scenarios of the same studies (black dot) in percentage. Caloric consumption across scenarios is normalised to $2000 \mathrm{kcal}$ to facilitate comparison. 


\section{Appendix B}

B1 menuCH data

B2 Environmental and socio-economic impact assessment

B3 Statistical analysis 
I 12 | Appendix B

\section{B I menuCH data}

Table BI.I. Characteristics of the menuCH study participants with 2 complete 24 h dietary recalls $(n=2,057)$ (see also Krieger et al. (20|9)).

\begin{tabular}{|c|c|c|}
\hline & Crude & Weighted $^{1}$ \\
\hline \multicolumn{3}{|l|}{ Sex } \\
\hline Males & $45.4 \%$ & $49.8 \%$ \\
\hline Females & $54.6 \%$ & $50.2 \%$ \\
\hline \multicolumn{3}{|l|}{ Age group } \\
\hline $18-29$ years & $19.4 \%$ & $18.8 \%$ \\
\hline 30-44 years & $25.9 \%$ & $29.9 \%$ \\
\hline $45-59$ years & $30.4 \%$ & $29.8 \%$ \\
\hline $60-75$ years & $24.3 \%$ & $21.6 \%$ \\
\hline \multicolumn{3}{|l|}{ Body Mass Index (BMI) group } \\
\hline Underweight $\left(\mathrm{BMI}<18.5 \mathrm{~kg} / \mathrm{m}^{2}\right)$ & $2.5 \%$ & $2.4 \%$ \\
\hline Normal weight $\left(18.5 \leq \mathrm{BMI}<25 \mathrm{~kg} / \mathrm{m}^{2}\right)$ & $54.2 \%$ & $54.1 \%$ \\
\hline Overweight $\left(25 \leq \mathrm{BMI}<30 \mathrm{~kg} / \mathrm{m}^{2}\right)$ & $30.6 \%$ & $30.6 \%$ \\
\hline Obese $\left(\mathrm{BMI} \geq 30 \mathrm{~kg} / \mathrm{m}^{2}\right)$ & $12.7 \%$ & $12.9 \%$ \\
\hline \multicolumn{3}{|l|}{ Language region } \\
\hline German-speaking & $65.2 \%$ & $69.2 \%$ \\
\hline French-speaking & $24.4 \%$ & $25.2 \%$ \\
\hline Italian-speaking & $10.4 \%$ & $5.6 \%$ \\
\hline \multicolumn{3}{|l|}{ Nationality } \\
\hline Swiss only & $72.5 \%$ & $61.4 \%$ \\
\hline Swiss binational & $14.4 \%$ & $13.8 \%$ \\
\hline Non-Swiss & $13.0 \%$ & $24.8 \%$ \\
\hline \multicolumn{3}{|l|}{ Education; highest degree } \\
\hline Primary school or no degree & $4.3 \%$ & $4.7 \%$ \\
\hline Secondary & $47.1 \%$ & $42.6 \%$ \\
\hline Tertiary & $48.5 \%$ & $52.6 \%$ \\
\hline \multicolumn{3}{|l|}{ Civil status } \\
\hline Single & $30.8 \%$ & $31.1 \%$ \\
\hline Married or in registered partnership & $54.7 \%$ & $52.2 \%$ \\
\hline Divorced or terminated partnership & $10.8 \%$ & $12.1 \%$ \\
\hline Other & $3.5 \%$ & $4.4 \%$ \\
\hline \multicolumn{3}{|l|}{ Income group } \\
\hline$<6,000 \mathrm{CHF} /$ month & $16.8 \%$ & $17.7 \%$ \\
\hline 6,000 to $13,000 \mathrm{CHF} /$ month & $40.9 \%$ & $39.8 \%$ \\
\hline$>13,000 \mathrm{CHF} /$ month & $13.9 \%$ & $14.9 \%$ \\
\hline Did not answer & $28.4 \%$ & $27.6 \%$ \\
\hline \multicolumn{3}{|l|}{ Smoking status } \\
\hline No smoker & $44.4 \%$ & $42.9 \%$ \\
\hline Former & $33.4 \%$ & $33.6 \%$ \\
\hline Current & $21.9 \%$ & $23.3 \%$ \\
\hline
\end{tabular}

\footnotetext{
${ }^{1}$ Weighted for sex, age, civil status, major area, household size, and nationality (\%; sum of weights $n=4,627,878$ ).
} 


\begin{tabular}{lrr}
\hline Currently on a weight-loss diet & & \\
No & $94.3 \%$ & $94.4 \%$ \\
Yes & $5.5 \%$ & $5.4 \%$ \\
\hline
\end{tabular}

Table B I.2. Average consumption per food group of the menuCH study participants (in primary product equivalents) (see also Chatelan et al. (2017b)).

\begin{tabular}{lr}
\hline & $\begin{array}{c}\text { Average consumption } \\
\text { per food group (in gram) }\end{array}$ \\
\hline Vegetables and fruits & 489.10 \\
Cereals and potatoes & 253.28 \\
Dairy and eggs & 330.17 \\
Meat, fish and seafood & 139.30 \\
Pulses, nuts and seeds & 14.60 \\
Oils and fats & 34.99 \\
Sugar, other sweeteners & 70.38 \\
Other animal products & 65.23 \\
Other vegetal products & 40.18 \\
\hline
\end{tabular}

The dietary recall data menuCH is generally available for researchers by application to the host institution, the Swiss Federal Food Safety and Veterinary Office.

\section{Transformations menuCH data}

In the menuCH dietary recall dataset (Chatelan et al., 2017a; Pestoni et al., 2019), amounts are provided ready-to-consume (cooked and processed, if applicable), and additionally, mixed foods (e.g. Pizza) are disentangled to ingredients (e.g. dough, tomatoes, and soft cheese). For this study, the amounts per ingredients (thus, e.g. dough, tomatoes, and soft cheese) were chosen for the further assessment. Of these ingredients, 30 still needed to be further disentangled into primary ingredients, such as dough to wheat flour, water, and salt (Table B1.3). For this, additional recipes were added, based on prevailing compositions of these foods, which led to a total of 110 food items. These food items were then mapped and aggregated to the commodities used in SOLm, which led to a total of 94 commodities. Then, for these commodities, the following steps were required in order to get from consumed values to commodities: cooked amounts were converted to raw amounts, and water content for coffee and tea products adjusted (Table B1.4). Further, not else specified categories, such as unspecified meat, were allocated to specific categories according to current shares in domestically available quantity (FAOSTAT, 2018). In order to account for losses along the production chain, food waste factors from Beretta et al. (2017) were added.

Table BI.3. Disentangling mixed ingredients (Product menuCH) into primary ingredients.

\begin{tabular}{lll}
\hline Product menuCH & Product Ingredient & Factor \\
\hline Apérogebäck n.s. & Mehl & 0.40 \\
Apérogebäck n.s. & Ei & 0.09 \\
Apérogebäck n.s. & Wasser & 0.30 \\
Apérogebäck n.s. & Salz n.s. & 0.01 \\
Apérogebäck n.s. & Gemüse n.s. & 0.10 \\
Apérogebäck n.s. & Fleisch n.s. & 0.10 \\
Bonbon & Zucker n.s. & 0.50
\end{tabular}


I I4 | Appendix B

Bonbon

Bonbon

Brot n.s.

Brot n.s.

Brot n.s.

Brot, Dinkel

Brot, Dinkel

Brot, Dinkel

Cappuccino, mit Koffein

Cappuccino, mit Koffein

Crème

Crème

Crème

Fleischersatz

Fleischersatz

Fleischersatz

Frucht- und Gemüsesäfte

Frucht- und Gemüsesäfte

Frühstückscerealien

Frühstückscerealien

Getreideriegel

Getreideriegel

Gipfeli, Butter

Gipfeli, Butter

Gipfeli, Butter

Glace n.s.

Glace n.s.

Glace n.s.

Guetzli n.s.

Guetzli n.s.

Guetzli n.s.

Guetzli n.s.

Guetzli n.s.

Knäckebrot

Knäckebrot

Konfitüre

Konfitüre

Kuchen n.s.

Kuchen n.s.

Kuchen n.s.

Kuchen n.s.

Kuchen n.s.

Kuchen n.s.

Milchmischgetränk

Milchmischgetränk

Milchreis

Milchreis

Salzige Saucen

Salzige Saucen

Salzige Saucen

Schokolade n.s.

Schokolade n.s.

Schokolade n.s.

Süssstoff n.s. $\quad 0.4$

Wasser $\quad 0.10$

Mehl $\quad 0.50$

Wasser $\quad 0.48$

Salz n.s. $\quad 0.02$

Mehl, Dinkel $\quad 0.50$

Wasser $\quad 0.48$

Salz n.s. $\quad 0.02$

Kaffee, mit Koffein $\quad 0.50$

Milch n.s. $\quad 0.50$

Rahm n.s. $\quad 0.55$

Zucker n.s. $\quad 0.35$

Ei 0.10

Tofu $\quad 0.50$

Pilze n.s. $\quad 0.48$

Salz n.s. $\quad 0.02$

Gemüsesaft $\quad 0.01$

Fruchtsaft $\quad 0.99$

Getreidekörner n.s. $\quad 0.90$

Zucker n.s. $\quad 0.10$

Getreidekörner n.s. $\quad 0.80$

Zucker n.s. $\quad 0.20$

Mehl $\quad 0.50$

Butter $\quad 0.30$

Wasser $\quad 0.20$

Wasser $\quad 0.40$

Rahm n.s. $\quad 0.30$

Zucker n.s. $\quad 0.30$

Mehl $\quad 0.40$

Zucker, Kristall $\quad 0.25$

Fett n.s. $\quad 0.25$

Ei 0.10

Salz n.s. $\quad 0.01$

Getreidekörner n.s. 0.95

Salz n.s. $\quad 0.05$

Zucker, Kristall $\quad 0.50$

Früchte $\quad 0.50$

Mehl $\quad 0.30$

Butter $\quad 0.20$

Zucker, Kristall $\quad 0.30$

Ei $\quad 0.15$

Kakao $\quad 0.03$

Früchte $\quad 0.03$

Milch n.s. $\quad 0.70$

Zucker n.s. $\quad 0.30$

Milch n.s. $\quad 0.50$

Reis n.s. $\quad 0.50$

Wasser $\quad 0.60$

Rahm n.s. $\quad 0.35$

Salz n.s. $\quad 0.05$

Kakao $\quad 0.45$

Milch n.s. $\quad 0.15$

Zucker n.s. $\quad 0.40$ 


\begin{tabular}{|c|c|c|}
\hline Sirup & Wasser & 0.85 \\
\hline Sirup & Zucker n.s. & 0.15 \\
\hline Sirup (Konzentrat) & Wasser & 0.30 \\
\hline Sirup (Konzentrat) & Zucker n.s. & 0.70 \\
\hline Sojadrink & Wasser & 0.80 \\
\hline Sojadrink & Flocken, Soja & 0.10 \\
\hline Sojadrink & Zucker n.s. & 0.10 \\
\hline Suppe n.s. & Wasser & 0.59 \\
\hline Suppe n.s. & Gemüse n.s. & 0.30 \\
\hline Suppe n.s. & Mehl & 0.10 \\
\hline Suppe n.s. & Salz n.s. & 0.01 \\
\hline Süssgetränk & Wasser & 0.85 \\
\hline Süssgetränk & Zucker n.s. & 0.15 \\
\hline Teig n.s. & Wasser & 0.40 \\
\hline Teig n.s. & Mehl & 0.50 \\
\hline Teig n.s. & Öl n.s. & 0.10 \\
\hline Teigwaren n.s. & Mehl & 0.68 \\
\hline Teigwaren n.s. & $\mathrm{Ei}$ & 0.30 \\
\hline Teigwaren n.s. & Salz n.s. & 0.02 \\
\hline Teigwaren, Ravioli, Fleischfüllung & Mehl & 0.38 \\
\hline Teigwaren, Ravioli, Fleischfüllung & $\mathrm{Ei}$ & 0.20 \\
\hline Teigwaren, Ravioli, Fleischfüllung & Salz n.s. & 0.02 \\
\hline Teigwaren, Ravioli, Fleischfüllung & Käse n.s. & 0.20 \\
\hline Teigwaren, Ravioli, Fleischfüllung & Fleisch n.s. & 0.20 \\
\hline Teigwaren, Ravioli, Spinat + Ricotta & Mehl & 0.38 \\
\hline Teigwaren, Ravioli, Spinat + Ricotta & $\mathrm{Ei}$ & 0.20 \\
\hline Teigwaren, Ravioli, Spinat + Ricotta & Salz n.s. & 0.02 \\
\hline Teigwaren, Ravioli, Spinat + Ricotta & Weichkäse n.s. & 0.20 \\
\hline Teigwaren, Ravioli, Spinat + Ricotta & Gemüse n.s. & 0.20 \\
\hline Wasserglace & Zucker n.s. & 0.20 \\
\hline Wasserglace & Wasser & 0.80 \\
\hline
\end{tabular}

Table BI.4. Factors to convert cooked amounts into raw amounts and to adjust water content (coffee and tea).

\begin{tabular}{ll}
\hline Food item & Conversion factor \\
\hline Chick peas & 0.40 \\
Lentils & 0.40 \\
Quinoa & 0.40 \\
Pulses & 0.40 \\
Beans & 0.40 \\
Coffee and products & 0.06 \\
Rice (milled equivalent) & 0.33 \\
Soyabeans & 0.40 \\
Tea (including mate) & 0.01 \\
Seasoning & 0.10 \\
\hline
\end{tabular}




\section{6 | Appendix B}

\section{B2 Environmental and socio-economic impact assessment}

\section{Environmental impact assessment}

SOLm is a biophysical mass- and nutrient-flow model (Muller et al., 2017; Schader et al., 2015) that up to now has mainly been used to compute multiple environmental impacts of the global food system, either in its current state, or for changed future states. A detailed description of the model as well as code files can be found in deliverable D4.1 of the EU-project UNISECO, which is available in an updated form from the online repository 'orgprints' (Muller et al., 2020), and in the supplementary material of two previous publications that used this model (Muller et al., 2017; Schader et al., 2015). SOLm is set up such that analyses are able both at global and at regional or national scales. The default data for all countries are the production and consumption data as provided by the FAO in FAOSTAT (FAOSTAT, 2018). In this study, we extended the application of SOLm to impact intensities on product level (Table B2.1). For this, we implemented additional modules to be able to calculate impacts per consumed product. More concretely, we added modules on trade and transport, commodity trees, and allocation. The trade and transport module is based on trade data from (FAOSTAT, 2018), which we adjusted according to an approach proposed in Kastner et al. (2011) in order to correct for pass-through stages during the transport of products. Distances to Switzerland were estimated based on ecotransit.org for different modes of transport. Further, commodities were categorised into perishable and non-perishable products, and depending on the perishability and the estimated transporting distance, the mode of transport was chosen. More concretely, for distances below or equal to $5000 \mathrm{~km}$, lorry was chosen as transport mode. Transports above $5000 \mathrm{~km}$ were treated differently depending on perishability: non-perishable products were assumed to be transported by ship and lorry (the majority), and perishable products (such as certain fruits) were assumed to be transported by aircraft and lorry (Stolze et al., 2019a). Inventories per transport mode were taken from Ecoinvent 3. Thus, greenhouse gas (GHG) emissions from transport were included for feed and for final commodities, based on approximate regions of origin of Swiss food consumption. Further, GHG emissions from processing were included for the following products: processed meat, fruit and vegetable products, oils and fats, dairy products, grain mill and starch products, bakery products, sugar, chocolate, and beverages (Stolze et al., 2019a). Then, because fish products are not covered in SOLm, we applied European averages of global warming potential (GWP) impact intensities from Poore and Nemecek (2018) for this food group.

GHG calculations from agricultural production in SOLm are done according to the IPCC guidelines for GHG inventories from 2006 (IPCC, 2006a). These thus cover emissions from enteric fermentation, manure management, fertilizer application, management of organic soils, cultivation of rice and open biomass burning. Specifically for Switzerland, more detailed data for various key parameters for the different emission calculations are used if available from the Swiss national GHG inventory (FOEN, 2019). This covers, for example, specific values on quantity- and nitrogen-contents-based shares of different manure management systems for the different livestock types, specific values for $\mathrm{N}$-volatilisation rates from manure management, emission factors for organic soils, etc. 
In SOLm, the fertilizer application levels per hectare are based on the nutrient requirements of the plants. These nutrient requirements are provided by baseline or reference scenario data (e.g. from country-specific databases) (Muller et al., 2020). The nutrients available per region are then applied proportionally to these requirements. This applies for nitrogen in manure, residues, and mineral fertilizers, and for phosphorus in mineral fertilizers. Phosphorus in manure and residues is calculated from the quantities of manure and residues applied, which in turn are derived based on the nitrogen application in these fertilizers (Muller et al., 2020).

Table B2.I. Climate change and land use impact intensities per food item.

\begin{tabular}{|c|c|c|c|}
\hline Food item & $\begin{array}{l}\text { Total impacts per } \\
\mathrm{kg} \text { of food item } \\
(\mathrm{kg} \mathrm{CO} \text { eq) }\end{array}$ & $\begin{array}{l}\text { Total impacts per } \\
\mathrm{kg} \text { of food item } \\
\left(\text { cropland, } \mathrm{m}^{2}\right)\end{array}$ & $\begin{array}{l}\text { Total impacts per } \\
\mathrm{kg} \text { of food item } \\
\left(\text { grassland }, \mathrm{m}^{2}\right)\end{array}$ \\
\hline \multicolumn{4}{|l|}{ Cereal and potatoes } \\
\hline Wheat and products & 1.10 & 1.71 & 0.0 \\
\hline Flour, wheat & 1.42 & 2.25 & 0.0 \\
\hline Maize and products & 0.93 & 1.08 & 0.0 \\
\hline Rice (milled equivalent) & 3.23 & 2.43 & 0.0 \\
\hline Quinoa & 0.90 & 11.92 & 0.0 \\
\hline Oats & 0.64 & 1.93 & 0.0 \\
\hline Cereals - Excluding Beer & 1.10 & 1.71 & 0.0 \\
\hline Millet and products & 1.08 & 3.80 & 0.0 \\
\hline Potatoes and products & 0.27 & 0.49 & 0.0 \\
\hline \multicolumn{4}{|l|}{ Fruits and vegetables } \\
\hline Fruits and berries & 0.86 & 3.11 & 0.0 \\
\hline Grapes and products (excl. wine) & 0.39 & 1.15 & 0.0 \\
\hline Bananas & 0.33 & 0.44 & 0.0 \\
\hline Oranges, Mandarines & 0.52 & 0.64 & 0.0 \\
\hline Lemons, Limes and products & 0.51 & 0.98 & 0.0 \\
\hline Grapefruit and products & 0.49 & 0.42 & 0.0 \\
\hline Cabbages and other brassicas & 0.34 & 0.62 & 0.0 \\
\hline Leafy vegetables & 0.37 & 0.72 & 0.0 \\
\hline Branch vegetables & 0.32 & 0.49 & 0.0 \\
\hline Fruit vegetables & 0.17 & 0.05 & 0.0 \\
\hline Pumpkins, squash and gourds & 0.29 & 0.51 & 0.0 \\
\hline Peas & 0.64 & 2.59 & 0.0 \\
\hline \multicolumn{4}{|l|}{ Sugars } \\
\hline Sugar beet & 0.26 & 0.12 & 0.0 \\
\hline Sugar cane & 0.39 & 0.13 & 0.0 \\
\hline \multicolumn{4}{|l|}{ Pulses, nuts, and seeds } \\
\hline Soyabeans & 0.62 & 3.63 & 0.0 \\
\hline Chick peas & 0.61 & 7.52 & 0.0 \\
\hline Lentils & 0.68 & 9.81 & 0.0 \\
\hline Beans & 0.71 & 5.84 & 0.0 \\
\hline Almonds, shelled & 1.04 & 6.84 & 0.0 \\
\hline Groundnuts, shelled & 1.67 & 8.71 & 0.0 \\
\hline Hazelnuts, shelled & 1.32 & 19.84 & 0.0 \\
\hline Walnuts, shelled & 6.22 & 16.26 & 0.0 \\
\hline Sunflower seed & 2.02 & 3.59 & 0.0 \\
\hline \multicolumn{4}{|l|}{ Meat } \\
\hline Meat, cattle & 13.17 & 10.66 & 8.76 \\
\hline
\end{tabular}




\begin{tabular}{lrrr} 
Meat, beef and veal sausages & 16.45 & 13.32 & 10.94 \\
Meat, cattle, boneless (beef \& & 18.53 & 15.01 & 12.33 \\
veal) & & & \\
Pigmeat & 6.00 & 10.33 & 0.0 \\
Meat, chicken & 2.23 & 8.90 & 0.0 \\
\hline Milk, milk products, and eggs & & & 1.06 \\
\hline Milk, Whole & 2.24 & 1.29 & 6.22 \\
Cheese, whole cow milk & 6.40 & 7.58 & 10.58 \\
Hard cheese & 10.86 & 12.88 & 6.61 \\
Semihard cheese & 6.80 & 8.05 & 4.81 \\
Soft cheese & 4.95 & 5.85 & 11.26 \\
Butter, Ghee & 11.53 & 13.70 & 1.32 \\
Yoghurt & 2.78 & 1.61 & 7.05 \\
Cream & 7.25 & 8.59 & 0.0 \\
Eggs & 2.23 & 8.95 & 0.0 \\
\hline Oils & & & 0.0 \\
\hline Rape and Mustard Oil & 4.13 & 8.47 & 0.0 \\
Olive oil & 1.49 & 23.04 & 0.0 \\
\hline Miscellaneous & & & 0.0 \\
\hline Cocoa beans and products & 4.32 & 20.30 & 0.0 \\
Coffee and products & 0.64 & 12.00 & 0.0 \\
Tea (including mate) & 1.23 & 6.77 & \\
Wine & 0.54 & 1.71 & 0.35 \\
Beer & 0.30 & & \\
\hline
\end{tabular}

\section{Social risks}

The Social Hotspots Database (SHDB) consists of 156 social indicators from 22 themes within five Social Impact Categories (Labor Rights and Decent Work, Health and Safety, Human Rights, Governance, and Community Infrastructure) (Norris et al., 2014). Underlying the SHDB, a global Input Output model, originating from the Global Trade Analysis Project GTAP, in combination with a Worker Hours Model, were employed. With these two models and Social Theme Tables, the 156 social indicators were derived. These indicators, available per country and per sector, are aggregated according to a proposed weighting scheme to the Social Hotspot Index (SHI) (Benoit-Norris et al., 2012; Norris et al., 2014). In the SHI, only negative impacts are included. First, levels of risks as available in the SHDB are employed, ranging from 0.1 (low risk) to 10 (very high risk). Then, social issues are weighted according to a proposed weighting scheme (Benoit-Norris et al., 2012; Norris et al., 2014), and finally, by summing over the product of weights and risk levels, the SHI is obtained.

\section{Price data}

Price data were collected from $\underline{w w w . c o o p . c h}$ on the 15.10.2019.

Table B2.2. Price data (CHF per $\mathrm{kg}$ of food item).

\begin{tabular}{ll}
\hline Food item & Price per kg of food item (CHF) \\
\hline Bananas & 1.40 \\
Berries nes & 23.60 \\
Cabbages and other brassicas & 4.20 \\
Chestnut & 14.00 \\
Chick peas & 4.90
\end{tabular}


Dates $\quad 11.70$

Ginger $\quad 8.00$

Lentils $\quad 5.00$

Mushrooms and truffles $\quad 13.20$

Oats $\quad 3.90$

Pumpkins, squash and gourds $\quad 3.95$

Quinoa $\quad 12.40$

Sesame seed $\quad 11.20$

Sugar beet $\quad 1.00$

Sugar cane $\quad 2.20$

Sunflower seed $\quad 6.30$

Wine $\quad 17.00$

Meat, chicken $\quad 14.50$

Meat, duck $\quad 38.00$

Meat, game $\quad 45.00$

Meat, turkey $\quad 25.00$

Yoghurt 2.40

Almonds shelled $\quad 15.00$

Bulgur $\quad 5.90$

Cashew nuts, shelled $\quad 19.80$

Fat, nes, prepared $\quad 0.00$

Flour, wheat $\quad 1.85$

Groundnuts, shelled $\quad 3.15$

Hazelnuts, shelled $\quad 17.30$

Juice, fruit nes $\quad 1.40$

Meat, beef and veal sausages $\quad 25.00$

Meat, cattle, boneless (beef \& veal) 80.00

Soya curd $\quad 5.90$

Walnuts, shelled $\quad 9.00$

Animal fats
Beer

Onions $\quad 1.30$

Pigmeat $\quad 27.00$

Pulses $\quad 5.00$

Beans $\quad 9.20$

Beverages, Alcoholic $\quad 15.00$

Cereals - Excluding Beer $\quad 5.60$

Cocoa Beans and products $\quad 15.20$

Coconut Oil $\quad 13.20$

Coconuts - Incl Copra $\quad 2.00$

Coffee and products $\quad 13.90$

Fruits - Excluding Wine $\quad 6.50$

Grapefruit and products $\quad 4.80$

Grapes and products (excl wine) $\quad 5.00$

Lemons, Limes and products $\quad 6.60$

Maize and products $\quad 3.50$

Millet and products $\quad 9.00$

Nuts and products $\quad 5.60$

Oilcrops $\quad 4.30$

Oilcrops Oil, Other $\quad 4.30$

Olive Oil $\quad 7.50$

Olives (including preserved) $\quad 3.90$

Oranges, Mandarines $\quad 2.95$ 


\begin{tabular}{ll} 
Peas & 5.30 \\
Potatoes and products & 1.98 \\
Rape and Mustard Oil & 4.30 \\
Rice (Milled Equivalent) & 2.50 \\
Soyabeans & 13.80 \\
Sugar (Raw Equivalent) & 1.00 \\
Sweeteners, Other & 11.20 \\
Tea (including mate) & 12.50 \\
Vegetables & 10.00 \\
Wheat and products & 2.95 \\
Butter, Ghee & 12.20 \\
Cheese & 25.00 \\
Cream & 6.60 \\
Eggs & 5.92 \\
Fish, Seafood & 37.50 \\
Freshwater Fish & 38.00 \\
Honey & 9.80 \\
Marine Fish, Other & 26.70 \\
Milk, Skimmed & 1.53 \\
Milk, Whole & 1.60 \\
Mutton \& Goat Meat & 36.00 \\
Pelagic Fish & 19.50 \\
Flour, Spelt & 3.60 \\
Salt & 1.70 \\
Water & 0.63 \\
Hard cheese & 25.00 \\
Semihard cheese & 28.00 \\
Soft cheese & 30.00 \\
Leafy vegetables & 20.00 \\
Branch vegetables & 6.50 \\
Fruit vegetables & 8.00 \\
Root vegetables & 4.20 \\
\hline
\end{tabular}

\section{Dichotomisation}

We calculated dichotomised impact intensities of all impact categories in order to facilitate the interpretation of each food group's strength. For the impact categories GWP, LO, SHI, and cost, impact intensities per food group could simply be divided into above and below median. For this, we first calculated the median per impact category. Then, each food group was allocated to below or above median according to their impact intensity value. However, as the Alternate Healthy Eating Index (AHEI) is not computed per food group but at diet level (Chiuve et al., 2012), additional assumptions were required to divide food groups into weak or strong for this impact category. Instead of above and below median, therefore, we based our dichotomisation on whether food groups clearly contribute to an increase of the AHEI (strong) or to a decrease or unclear effect on the AHEI (weak). In the following, we explain this process for each food group.

Red and processed meats do not contribute to an increase of the AHEI. In fact, the component 'red and processed meat' receives a higher score when consumption is lower (Chiuve et al., 2012). Therefore, we assign the food groups cattle meat and pork to the weak group. In addition, since chicken only contributes potentially weakly to an increase of the AHEI, also the third meat food 
group is classified as weak. The food groups milk products and eggs do not contribute to an increase of the AHEI, and are therefore classified as weak as well. Fish and seafood, on the other hand, contribute via the component 'long-chain (n-3) fats' (eicosapentaenoic EPA and docosahexaenoic DHA) to an increase of the AHEI, and are therefore classified as strong (Chiuve et al., 2012). The food group cereals contributes to an increase of the AHEI, if whole grain products are consumed (Chiuve et al., 2012). Therefore, this food group is classified as strong. Moreover, potatoes are not listed to increase the AHEI, and are hence classified as weak. The food groups pulses, vegetables, nuts and seeds, and fruits, on the contrary, contribute to an increase of the AHEI via the components vegetables, nuts and legumes, and fruit. As a consequence, these three food groups are classified as strong. The category of oils and fats could, on the one hand, increase the AHEI via the component poly-unsaturated fatty acid, and on the other hand, reduce the AHEI via the component trans-fat. Thus, for this food group, the quality and quantity largely drive the direction of the effect on the AHEI. Due to the broad coverage of the food group oils and fats, we hence decided to classify this as weak. Further, sugar is only represented in the AHEI via sugar-sweetened beverages. There, the lower the consumption, the higher the score. As this is reasonable for all types of sugar intake, we classify sugar as weak. Finally, the food group alcoholic beverages, does, if consumed in moderate amounts, contribute to an increase of the AHEI (Chiuve et al., 2012). However, since the threshold for moderate amounts is relatively narrow, and everything above this again decreases the score, we classified alcoholic beverages as weak.

\section{B3 Statistical analysis}

Associations between sociodemographic and lifestyle factors and food choices were evaluated using multiple linear regression models with ordinary least squares estimators. The sociodemographic and lifestyle factors, all categorical, were employed as explanatory variables. Hence, consumed amounts per food group were employed as dependent variables. In order to reveal associations for each food group separately, one linear regression model was fitted per food group. Differences in levels of caloric intake were considered by including caloric intake as an adjusting factor. These models took the following form:

$$
\text { FI estimate } \text { es }_{i}=\beta_{0}+\beta * S L F_{j}+\beta^{\prime} * C I+\varepsilon
$$

where FI estimatei represents estimates of intake for food $i$; $S_{L F}$ represents the categorical sociodemographic and lifestyle factors $j$, and CI represents caloric intake. VIFs were tested to inspect multi-collinearity, with all scores being below 2.5, thus not indicating potential multi-collinearity (O'brien, 2007). With this analysis, we thus investigated associations between for example being male, as compared to being female, and cattle meat intake, while keeping all other sociodemographic and lifestyle factors constant.

Associations between sociodemographic and lifestyle factors and sustainability impacts were evaluated in the same manner as for food choices. Thus, these took the following form:

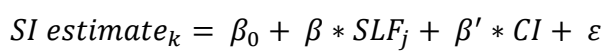

where SI estimatek represents estimates of sustainability impacts k; SLF represents the categorical sociodemographic and lifestyle factors $\mathrm{j}$, and CI represents caloric intake. 


\section{2 | Appendix B}

The statistical analyses were conducted using the $\mathrm{R}$ software (version 3.6.1 for Windows, $\mathrm{R}$ Foundation for Statistical Computing, Vienna, Austria). 


\title{
Appendix C
}

\author{
C1 Consumer strategies \\ C2 Environmental impact assessment \\ C3 Price data
}




\section{I24 | Appendix C}

\section{Consumer strategies}

\section{Development of strategies}

Within the project "Sustainable and healthy diets: Trade-offs and synergies", two stakeholder workshops took place to develop scenarios for a more sustainable future Swiss food system (Stolze et al., 2019a). There, stakeholders from the Federal administration of Switzerland, farmer associations, industry partners, retailers, consumer organisations, public health associations, and research took part. During the first workshop, ideas for scenarios were inventorised, leading to a broad compilation of aspects to be potentially considered in the project's scenarios.

These aspects were then combined into seven scenarios, of which two focused on human health (decrease of meat consumption, adhering to dietary guidelines), three focused on environmental sustainability (increase of organic produce, minimise greenhouse gas emissions, limit ruminant feed to grass resources with better closed nutrient cycles), and two focused on expectations and wishes of consumers (i.e. a large part of the production is regional and seasonal, and strict restrictions on animal welfare and animal health are implemented). In the second workshop, these scenarios were discussed with the stakeholders, and inputs regarding foci, potential trade-offs to consider, etc. were collected.

For this study, we rearranged the proposed scenarios to consumer strategies. Thereby, not all scenarios were considered due to modelling constraints (e.g. considering restrictions on animal welfare and animal health), while others, in particular reducing food waste at consumption stage, were added. The consumer strategies 1 and 2 were directly deduced from the human health focus; strategy 3 considered reduced food waste; strategy 4 targeted at increased regional production, which was implemented by increasing the share of domestic produce; strategy 5 targeted environmental sustainability by increasing the share of organic produce, and strategy 6 complemented strategy 5 with feeding restrictions on low-opportunity-cost biomass, thereby at the same time addressing the feed limit to grass resources. In addition, a reference strategy, which represents the status quo, was implemented.

\section{Additional specifications}

\section{Reference}

The reference strategy was calibrated according to data from the dietary recalls menuCH (Chatelan et al., 2017a). These dietary recalls represent most regions and age groups of Switzerland, but not all. For the remaining, scaling factors to estimate the consumption for whole Switzerland were applied accordingly. To derive production values from intake values (relevant for the environmental indicators and the social risks indicator), the same methodology was applied as in Frehner et al. (2021). In addition, cost data was employed at food demand level, thus including food waste at consumption stage.

\section{Strategy I: Meat reduction}

In this scenario, the complete meat consumption was reduced by $25 \%, 50 \%$, and $100 \%$, except fish and seafood. Pulses were increased to match protein contents of the reference strategy. 


\section{Strategy 2: Swiss Food Pyramid}

The Swiss Food Pyramid was specified according to Walter et al. (2007) and additional information from Von Ow et al. (2020) (Table C1.1). For implementation levels 25\% (and 50\%), we assumed diets composed by $25 \%$ (and 50\%) according to the Swiss Food Pyramid, and 75\% (and 50\%) according to the reference strategy.

Table CI.I. Specification of the Swiss Food Pyramid.

\begin{tabular}{|c|c|c|}
\hline Produktgruppe & $\begin{array}{l}\text { Nahrungsmittel } \\
\text { (Portionen) }\end{array}$ & $\begin{array}{l}\text { Menge (g) für } \\
\text { SFP-Szenario }\end{array}$ \\
\hline Gemüse \& Früchte & Gemüse (3) & 360 \\
\hline >täglich 5 Portionen & Früchte (2) & 240 \\
\hline Stärkeprodukte & Brot (1) & 100 \\
\hline >täglich 3 Portionen & Kartoffeln (2/7) & 68.6 \\
\hline > Brot weniger gut substituierbar (Frühstück), deshalb 1 Portion & Hülsenfrüchte (2/7) & 22.9 \\
\hline Brot & Hirse/Gerste (2/7) & 17.2 \\
\hline \multirow[t]{4}{*}{$>$ die restlichen Stärkelieferanten jeweils 2/7 Portionen } & Hafer $(2 / 7)$ & 17.2 \\
\hline & Mais $(2 / 7)$ & 17.2 \\
\hline & Teigwaren $(2 / 7)$ & 17.2 \\
\hline & Reis $(2 / 7)$ & 17.2 \\
\hline Milchprodukte & Milch (1) & 200 \\
\hline \multirow[t]{2}{*}{$>$ täglich 3 Portionen } & $\begin{array}{l}\text { Joghurt/Quark/Hüttenkäse } \\
\text { (1) }\end{array}$ & 180 \\
\hline & Halbhart-/Hartkäse (1) & 30 \\
\hline Proteinlieferanten & Rindfleisch (1/7) & 15.8 \\
\hline > täglich 1 Portion & Schweinefleisch (1/7) & 15.8 \\
\hline > zusätzliche Empfehlung pro Woche: Abwechslung zwischen & Pouletfleisch (1/7) & 15.8 \\
\hline Proteinlieferanten und max. 2-3 Portionen Fleisch, 1 Portion & Eier $(1 / 7)$ & 21.4 \\
\hline \multirow[t]{3}{*}{ Fisch, evtl. . Zusätzliche Portion Käse oder Quark möglich. } & Tofu $(1 / 7)$ & 15.8 \\
\hline & Fisch $(1 / 7)$ & 15.8 \\
\hline & Weichkäse (1/7) & 8.6 \\
\hline Öle, Fette & Rapsöl & 15 \\
\hline > täglich 20-30g Pflanzenöl, davon die Hälfte Rapsöl & Pflanzenöl & 10 \\
\hline > bei Bedarf täglich $10 \mathrm{~g}$ Butter o. $\ddot{A}$. & Butter & 5 \\
\hline $\begin{array}{l}\text { Nüsse, Kerne, Samen } \\
>\text { täglich } 1 \text { Portion }\end{array}$ & Nüsse & 25 \\
\hline Süsses, Salziges, Alkoholisches & Wein $(1 / 6)$ & 16.6 \\
\hline \multirow[t]{3}{*}{ > täglich max. eine Portion } & $\operatorname{Bier}(1 / 6)$ & 41.6 \\
\hline & Schokolade (1/3) & 6.6 \\
\hline & Chips $(1 / 3)$ & 6.6 \\
\hline Getränke & Wasser & 1500 \\
\hline
\end{tabular}

\section{Strategy 3: Food waste reduction}

Food waste fractions per food group were applied according to Beretta et al. (2017). For strategy 3, food waste at consumption stage was reduced by $25 \%$ and $50 \%$, respectively.

Strategy 4: Domestic produce

For strategy 4, domestic produce was increased to 50\%. Products that cannot be produced in Switzerland were compensated for within food groups. 


\section{I26 | Appendix C}

\section{Strategy 5 and 6: Organic produce and circular agriculture principles}

The increase in organic produce, and the addition with circular agriculture principles likewise, was implemented across food items. This change mainly affected the calculation of environmental impacts (Section C2) and dietary costs (Section C3).

\section{C2 Environmental impact assessment}

Global warming potential, land use, and nitrogen surplus were calculated using the biophysical mass- and nutrient-flow model SOLm model (Muller et al., 2020; Muller et al., 2017; Schader et al., 2015; Stolze et al., 2019a). In the online repository 'orgprints' (Muller et al., 2020), a detailed model description and code files can be found. SOLm is primarily calibrated with FAOSTAT data (FAOSTAT, 2018), which were refined for Switzerland (Frehner et al., 2021; Stolze et al., 2019b).

In strategy 6, feed for animals was limited to low-opportunity-cost biomass (LCB), i.e. biomass that is not used for direct human consumption and thus includes by-products, food waste, and grass resources. To consider this in the impact assessment, the allocation for processes with multiple outputs was adjusted, following the food-based allocation approach proposed in Van Hal et al. (2019b). Thereby, for by-products, impacts of the production stage were fully allocated to the main product, i.e. the food product. This assumption would however not hold if a limit to LCB on a broader scope, e.g. for a whole country, would be modelled. Then, also animal numbers would need to be reduced accordingly, such that the total LCB available is not exceeded.

\section{C3 Price data}

Price data were collected from www.coop.ch on the 03.09.2020. The price differences of organic products, compared to conventional products, differed substantially between food items (Table C3.1). Missing prices for organic products were filled with means per food group. 
Table C3.I. Price data (CHF per kg of food item).

\begin{tabular}{|c|c|c|}
\hline Food item & $\begin{array}{l}\text { Price per kg of food item, } \\
\text { conventional (CHF) }\end{array}$ & $\begin{array}{l}\text { Price per kg of food item, } \\
\text { organic (CHF) }\end{array}$ \\
\hline Bananas & 1.40 & 3.30 \\
\hline Berries nes & 23.60 & \\
\hline $\begin{array}{l}\text { Cabbages and other } \\
\text { brassicas }\end{array}$ & 4.20 & \\
\hline Chestnut & 14.00 & 15.90 \\
\hline Chick peas & 4.90 & 5.90 \\
\hline Dates & 11.70 & 13.20 \\
\hline Ginger & 8.00 & 23.0 \\
\hline Lentils & 5.00 & 5.90 \\
\hline Mushrooms and truffles & 13.20 & \\
\hline Oats & 3.90 & 4.00 \\
\hline $\begin{array}{l}\text { Pumpkins, squash and } \\
\text { gourds }\end{array}$ & 3.95 & \\
\hline Quinoa & 12.40 & 12.40 \\
\hline Sesame seed & 11.20 & 11.20 \\
\hline Sugar beet & 1.00 & 3.00 \\
\hline Sugar cane & 2.20 & 3.60 \\
\hline Sunflower seed & 6.30 & \\
\hline Wine & 17.00 & 22.00 \\
\hline Meat, chicken & 14.50 & 24.00 \\
\hline Meat, duck & 38.00 & \\
\hline Meat, game & 45.00 & \\
\hline Meat, turkey & 25.00 & \\
\hline Yoghurt & 2.40 & \\
\hline Almonds shelled & 15.00 & \\
\hline Bulgur & 5.90 & \\
\hline Cashew nuts, shelled & 19.80 & \\
\hline Fat, nes, prepared & 0.00 & \\
\hline Flour, wheat & 1.85 & \\
\hline Groundnuts, shelled & 3.15 & \\
\hline Hazelnuts, shelled & 17.30 & \\
\hline Juice, fruit nes & 1.40 & \\
\hline Meat, beef and veal sausages & 25.00 & \\
\hline $\begin{array}{l}\text { Meat, cattle, boneless (beef \& } \\
\text { veal) }\end{array}$ & 80.00 & 100.00 \\
\hline Soya curd & 5.90 & \\
\hline Walnuts, shelled & 9.00 & \\
\hline \multicolumn{3}{|l|}{ Animal fats } \\
\hline Beer & 3.50 & 5.00 \\
\hline Onions & 1.30 & \\
\hline Pigmeat & 27.00 & 40.00 \\
\hline Pulses & 5.00 & 5.90 \\
\hline Beans & 9.20 & \\
\hline Beverages, Alcoholic & 15.00 & \\
\hline Cereals - Excluding Beer & 5.60 & \\
\hline Cocoa Beans and products & 15.20 & \\
\hline Coconut Oil & 13.20 & \\
\hline Coconuts - Incl Copra & 2.00 & \\
\hline Coffee and products & 13.90 & \\
\hline
\end{tabular}




\section{I28 | Appendix C}

Fruits - Excluding Wine $\quad 6.50$

Grapefruit and products $\quad 4.80$

Grapes and products (excl $\quad 5.00$

wine)

Lemons, Limes and products 6.60

Maize and products $\quad 3.50$

Millet and products $\quad 9.00$

Nuts and products $\quad 5.60$

Oilcrops $\quad 4.30$

Oilcrops Oil, Other $\quad 4.30$

Olive Oil $\quad 7.50$

Olives (including preserved) $\quad 3.90$

Oranges, Mandarines $\quad 2.95$

Peas $\quad 5.30$

5.90

$\begin{array}{ll}1.98 & 3.20\end{array}$

Potatoes and products $\quad 1.98$

Rape and Mustard Oil $\quad 4.30$

Soyabeans $\quad 13.80$

Sugar (Raw Equivalent) $\quad 1.00$

Sweeteners, Other

11.20

Tea (including mate) $\quad 12.50$

Vegetables $\quad 10.00$

Wheat and products $\quad 2.95$

Butter, Ghee $\quad 12.20$

Cheese 25.00

Cream $\quad 6.60$

Eggs $\quad 5.92$

Fish, Seafood $\quad 37.50$

Freshwater Fish $\quad 38.00$

Honey $\quad 9.80$

Marine Fish, Other $\quad 26.70$

Milk, Skimmed $\quad 1.53$

2.10

$\begin{array}{ll}1.60 & 1.80\end{array}$

Milk, Whole $\quad 1.60$

Mutton \& Goat Meat $\quad 36.00$

Pelagic Fish $\quad 19.50$

Flour, Spelt $\quad 3.60$

Salt $\quad 1.70$

Water $\quad 0.63$

Hard cheese $\quad 25.00$

27.50

28.00

$30.00 \quad 26.80$

Soft cheese $\quad 30.00$

26.00

Leafy vegetables $\quad 20.00$

$\begin{array}{lll}\text { Branch vegetables } & 6.50 & 8.00 \\ \text { Fruit vegetables } & 8.00 & 9.70\end{array}$

$\begin{array}{lll}\text { Root vegetables } & 4.20 & 4.25\end{array}$ 


\section{Appendix D}

D1 Food-based dietary guidelines

D2 Calculation of low-opportunity-cost biomass

D3 Resource allocation model

D4 Environmental impact assessment 


\section{Food-based dietary guidelines}

\section{Selection of the case study countries}

After dividing Europe into five areas (Northern, Southern, Eastern, Western, and Central Europe), we chose one country from each area as representative for this area. This choice was done based on the following criteria, in the presented order: (i) availability of food-based dietary guidelines (FBDG) in English, (ii) level of detail provided, and (iii) most recently updated. With this, we derived the five case study countries: Sweden (representative for Northern Europe), Malta (representative for Southern Europe), Bulgaria (representative for Eastern Europe), the Netherlands (representative for Western Europe), and Switzerland (representative Central Europe).

Table DI.I. Overview of food-based dietary guidelines employed.

\begin{tabular}{|c|c|c|}
\hline Country & Food-based dietary guideline & Responsible Institution \\
\hline Bulgaria & $\begin{array}{l}\text { Food based dietary guidelines } \\
\text { for adults in Bulgaria }\end{array}$ & Ministry of Health \\
\hline Malta & $\begin{array}{l}\text { The healthy plate: Dietary } \\
\text { guidelines for Maltese adults }\end{array}$ & $\begin{array}{l}\text { The Health Promotion and Disease Prevention } \\
\text { Directorate, Parliamentary Secretariat for Health }\end{array}$ \\
\hline $\begin{array}{l}\text { The } \\
\text { Netherlands }\end{array}$ & The wheel of five & $\begin{array}{l}\text { Netherlands Nutrition Center (Het } \\
\text { Voedingscentrum) }\end{array}$ \\
\hline Sweden & $\begin{array}{l}\text { Find your way to eat greener, } \\
\text { not too much and to be active! }\end{array}$ & National Food Agency (Livsmedelsverket) \\
\hline Switzerland & The Swiss Food Pyramid & Swiss Society for Nutrition (SGE SSN) \\
\hline
\end{tabular}

\section{Translating food-based dietary guidelines into daily diets}

In order to translate these recommendations of the FBDGs, which are generally given at food group level, into a daily diet (per capita per day), we employed FAO Food Balance Sheets (FAO, 2001). The required additional assumptions were kept as close as possible to the dietary habits of the respective country. Of the FAO Food balance sheets, we used an average of the years 2007-2011, as these years contained the most complete data. Table D1.2 lists the division of food groups and food items relevant for the FBDGs of the five selected countries. Figure D1.1 illustrates the calculation process of translating FBDGs into daily diets, with the example of the food group "grains and potatoes" in the Dutch FBDG. Generally, in the food group 'grains', wheat was further divided into wheat flour, pasta, and other wheat products. Bread was excluded from this part, as it was listed as separate recommendation in all FBDGs used here. Moreover, for some FBDGs, not all food groups were sufficiently specified. There, we employed additional data sources to fill the gaps, see Table D1.3. In addition, since fish recommendations were not further specified, and our model included two farmed fish systems (Salmon and Tilapia) that represent other fish species with similar characteristics, we split fish recommendations equally between Salmon and Tilapia.

Further, we estimated the nutritional contribution of each FBDG diet using food composition tables. The quality of these tables differed between the five case study countries with regard to level of detail on nutrients and food items. To ensure a homogenous assessment of the nutritional 
contribution, we therefore used the same food composition tables for all countries. We selected the food composition table with the highest level of detail, which was the Dutch food composition table (NEVO-table, 2019).

Table DI.2. Division of food groups into food items. FBDG = food-based dietary guideline.

\begin{tabular}{|c|c|c|}
\hline $\begin{array}{l}\text { Food } \\
\text { group }\end{array}$ & Food item examples in FBDGs & Food items in the daily FBDG diets \\
\hline Grains & $\begin{array}{l}\text { Pasta, rice, couscous, breakfast } \\
\text { cereals, flour from grains (all } \\
\text { limitedly processed and wholegrain) }\end{array}$ & Wheat, maize, barley, oats, rye, rice \\
\hline Bread & Whole wheat bread & Whole wheat bread ${ }^{1}$ \\
\hline Potatoes & Potatoes & Potato, sweet potato \\
\hline Vegetables & All vegetables (fresh or frozen) & Tomatoes, onions, other vegetables \\
\hline Fruits & All fruits (fresh, dried or frozen) & $\begin{array}{l}\text { Apples, bananas, oranges and mandarins, lemons, limes } \\
\text { and products, grapefruit and products, citrus other, } \\
\text { plantains, pineapple and products, dates (dried), grapes } \\
\text { and products, fruits other }\end{array}$ \\
\hline Legumes & $\begin{array}{l}\text { All pulses (Fresh or canned } \\
\text { without salt) }\end{array}$ & Beans, soybeans, pulses other and products \\
\hline Dairy $^{2}$ & $\begin{array}{l}\text { Milk, yoghurt, cheese (low-fat, } \\
\text { unflavoured and/or low salt) }\end{array}$ & Milk, yoghurt, cheese \\
\hline Red meat & Beef, pork (unprocessed) & Bovine meat, pork \\
\hline $\begin{array}{l}\text { White } \\
\text { meat }\end{array}$ & Chicken, turkey (unprocessed) & Chicken \\
\hline Fish & $\begin{array}{l}\text { All types of fish (emphasis on } \\
\text { fatty fish) }\end{array}$ & Freshwater fish, marine fish, aquaculture (salmon, tilapia) \\
\hline Eggs & Eggs & Eggs \\
\hline Nuts & All nuts (unroasted and unsalted) & Mixed nuts and products, peanuts \\
\hline Seeds & All seeds (unsalted) & Sunflower seeds, sesame seeds \\
\hline $\begin{array}{l}\text { Oils and } \\
\text { fats }\end{array}$ & $\begin{array}{l}\text { All vegetable oils (emphasis on } \\
\text { olive oil and flaxseed oil) }\end{array}$ & $\begin{array}{l}\text { Peanut oil, sunflower oil, soybean oil, vegetable oil, olive } \\
\text { oil, rapeseed oil, oilcrops other }\end{array}$ \\
\hline Beverages $^{3}$ & $\begin{array}{l}\text { Water, coffee, tea, fresh fruit } \\
\text { juice, alcoholic beverages } \\
\text { (without added sugar) }\end{array}$ & Coffee, tea, fruit juice \\
\hline
\end{tabular}

Table DI.3. Data gaps encountered in FBDGs and alternative sources used. FBDG = food-based dietary guideline.

\begin{tabular}{lll}
\hline Country & Data gap & Alternative source \\
\hline Sweden & Recommendation for potatoes, eggs, and white & $\begin{array}{l}\text { Supplementary material from Röös et al. } \\
\text { meat }\end{array}$ \\
Sweden & Recommendation for cheese & Not replaced \\
Bulgaria & Recommendation for vegetable oils & FBDG from Albania and Greece \\
\hline
\end{tabular}




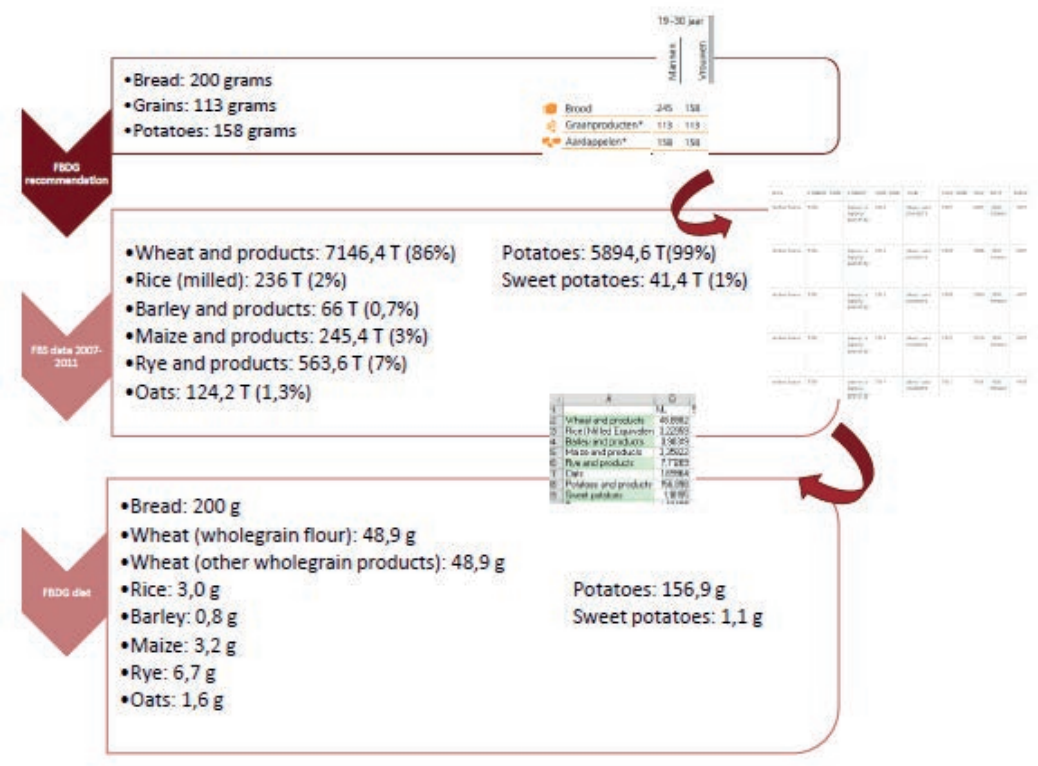

Figure DI.I. Process of translating FBDG recommendations into daily FBDG diets (example of the food group "grains and potatoes" of the Dutch FBDG).

\section{D2 Calculation of low-opportunity-cost biomass}

From the plant-source food part of the FBDGs, low-opportunity-cost biomass (LCB; including processing by-products, food waste, and grass resources) was calculated. If plant-source food requirements could not be covered by domestic production alone, additional plant-source food was imported, assuming their processing and consumption occurred within the country, leaving byproducts and food waste available as LCB. To estimate how much animal-source food (ASF) has to be produced to achieve recommended intakes, coefficients related to food loss and wastage, collected from Caldeira et al. (2019), were applied. Availability of food processing by-products as livestock feed were estimated using technical conversion factors (FAO, 2001). Available manufacturing waste in each country was estimated by employing data from the European Former Foodstuffs Processing Agency EFFPA (EFFPA, 2019). According to EFFPA, around 5 million tonnes of food waste at the manufacturing stage is currently fit to use as animal feed. By relating this to the total amount of waste leaving the manufacturing stage at EU level, we derived a fraction of food waste at the manufacturing stage. This fraction was then applied to the five case study countries. Moreover, to complete the available LCB, grass resources of the five countries were added (Van Selm et al., 2021).

\section{D3 Resource allocation model}

The resource allocation model was developed in General Algebraic Modelling System GAMS version 30.3. Previously, it has been employed for analyses on the potential for ASF based on current LCB on EU level (Van Hal, 2020; Van Hal et al., 2019a; Van Selm et al., 2021). In these assessments, 
human-digestible animal protein was maximised. We adapted the model in three aspects: first, we changed the scale from EU level to country level. Second, we expanded the objective function to include other nutrients besides protein. In the scenarios employed in this study, four different nutrient (groups) were maximised - human-digestible animal protein (MaxProt), the sum of all omega-3 fatty acids included (MaxFattyAcids), the sum of all minerals included (MaxMinerals), and the sum of all vitamins included (MaxVitamins). Third, as Switzerland was previously not included in the model, we added data for Switzerland from the same sources (Van Hal et al., 2019a; Van Selm et al., 2021).

\section{D4 Environmental impact assessment}

Global warming potential and land occupation were assessed using the bio-physical mass-flow model SOLm (Muller et al., 2017; Schader et al., 2015). This model depicts the main mass and nutrient flows of agricultural production. It covers 180 primary crop and 22 primary livestock activities, which are mainly calibrated with FAOSTAT data (FAOSTAT, 2018). Crop activities are characterised by the following inputs and outputs: land area (either occupied for crops or grass), mineral and organic fertiliser (manure, crop residues), nitrogen fixation, pesticides, management practices and processing for inputs, and crop yields (main and by-products) and residues for outputs. Further, livestock activities encompass the inputs feed and energy use for housing and fences, and the human-edible (meat, milk, eggs) and human-inedible (manure skins, hides, bones, etc.) outputs. Thus, covered emissions encompass feed production, enteric fermentation, manure management (further details: (Muller et al., 2017; Schader et al., 2015)).

In the scenarios where animal-source food was limited to LCB, we assumed that only resources that humans cannot or do not want to eat are fed. To reflect this, the calculation of environmental impact intensities was adjusted for multi outputs. Following the approach of food-based allocation proposed in Van Hal et al. (2019b), the full environmental impacts of crop production were allocated to the main plant-source food, which served as direct food for humans and was thus the driver of the production. Thus, covered emissions for animal-source food excluded feed production. It has to be noted that in reality, this is not necessarily the case, meaning that also co-products serving as feed can be an important driver for the actual production (e.g. soybean meal). Nevertheless, following the principle of circular food systems of feeding only LCB, we assumed that the use of by-products as feed is limited to those by-products that emerge from food-driven production. 


\section{Appendix E}

E1 Additional figures and tables 
I36 | Appendix E

\section{EI Additional figures and tables}

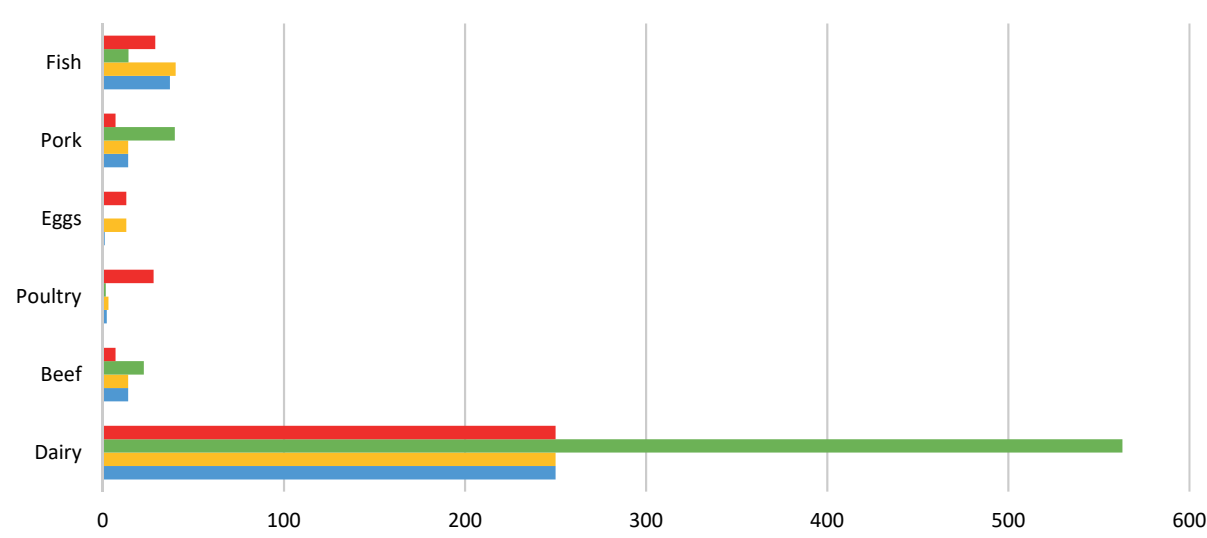

EAT-LANCET Reference $\quad$ EL Circular Wholegrain Potential EL Circular Refined-grain Fixed EL Circular Wholegrain Fixed

Figure EI.I. Quantities of animal-source food production from the three EL Circular scenarios and the EAT-Lancet reference scenario in grams per capita per day.

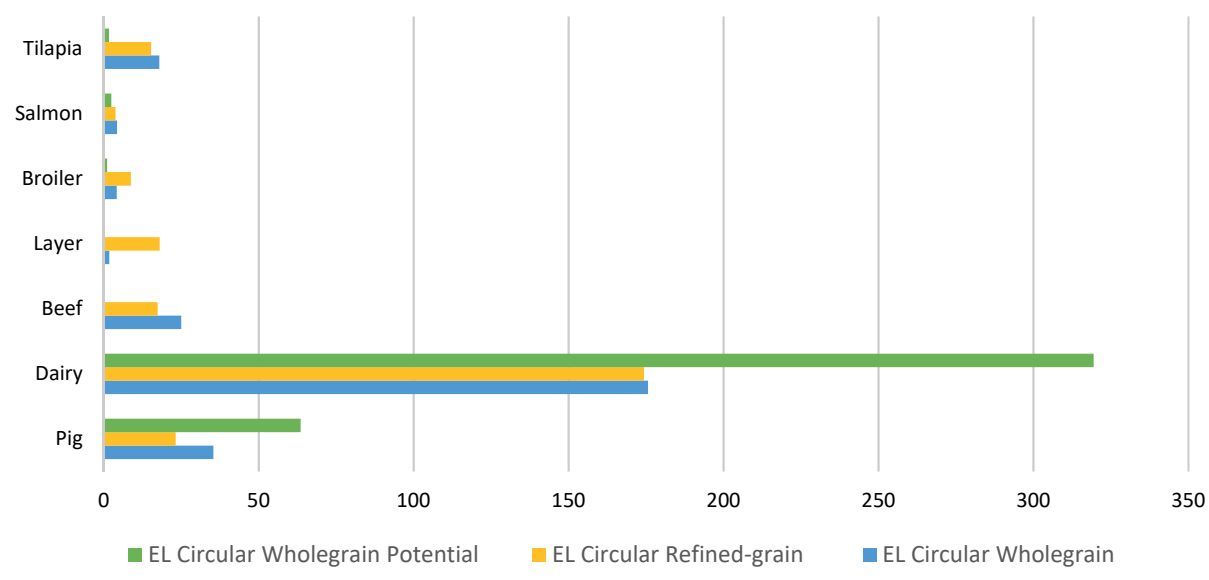

Figure EI.2. Greenhouse gas emissions in $\mathrm{CO}_{2}$ e per human capita per year from livestock in the three EL circular scenarios.

Table EI.I. Human nutrient requirements based on Average Requirements (AR) from the European Food Safety Authority used in Figure 3. *Vitamin $B_{12}$ is an adequate intake value and not an average requirement value.

\begin{tabular}{llll}
\hline Nutrient & Unit & Value & Additional information \\
\hline Energy & Kcal. & 2304 & Physical activity level = 1.6 \\
Protein & $\mathrm{G}$ & 46 & \\
Zinc & $\mathrm{Mg}$ & 16.9 & \\
Calcium & $\mathrm{Mg}$ & 750 & \\
Iron & $\mathrm{Mg}$ & 6 & \\
Vitamin B $12^{*}$ & $\mathrm{Ug}$ & 4 & \\
EPA and DHA & $\mathrm{G}$ & 0.25 & \\
\hline
\end{tabular}




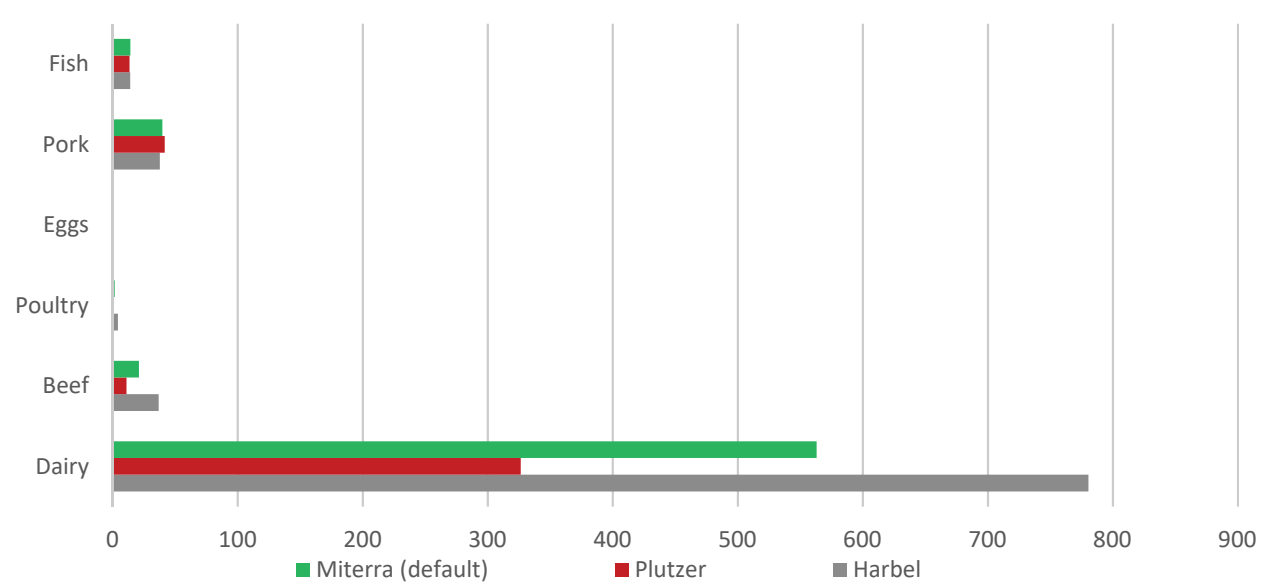

Figure EI.3. Area of managed grassland sensitivity: Range of animal-sourced food production in grams per capita per day. 
$138 \mid$ 


\section{References}

Abeliotis, K., Costarelli, V., Anagnostopoulos, K., 2016. The effect of different types of diet on greenhouse gas emissions in Greece. International Journal on Food System Dynamics 7(1), 3649.

Adesogan, A.T., Havelaar, A.H., McKune, S.L., Eilittä, M., Dahl, G.E., 2020. Animal source foods: Sustainability problem or malnutrition and sustainability solution? Perspective matters. Global Food Security 25, 100325.

Afshin, A., Sur, P.J., Fay, K.A., Cornaby, L., Ferrara, G., Salama, J.S., Mullany, E.C., Abate, K.H., Abbafati, C., Abebe, Z., 2019. Health effects of dietary risks in 195 countries, 1990-2017: a systematic analysis for the Global Burden of Disease Study 2017. The Lancet 393(10184), 19581972.

Aleksandrowicz, L., Green, R., Joy, E.J., Smith, P., Haines, A., 2016. The impacts of dietary change on greenhouse gas emissions, land use, water use, and health: a systematic review. PloS one 11(11), e0165797.

Andreyeva, T., Long, M.W., Brownell, K.D., 2010. The impact of food prices on consumption: a systematic review of research on the price elasticity of demand for food. American journal of public health 100(2), 216-222.

Anitha, S., Govindaraj, M., Kane-Potaka, J., 2020. Balanced amino acid and higher micronutrients in millets complements legumes for improved human dietary nutrition. Cereal Chemistry 97(1), 74-84.

Aprile, M.C., Caputo, V., Nayga Jr, R.M., 2012. Consumers' valuation of food quality labels: the case of the European geographic indication and organic farming labels. International Journal of Consumer Studies 36(2), 158-165.

Arrieta, E.M., González, A.D., 2018. Impact of current, National Dietary Guidelines and alternative diets on greenhouse gas emissions in Argentina. Food Policy 79, 58-66.

Asnicar, F., Berry, S.E., Valdes, A.M., Nguyen, L.H., Piccinno, G., Drew, D.A., Leeming, E., Gibson, R., Le Roy, C., Al Khatib, H., 2021. Microbiome connections with host metabolism and habitual diet from 1,098 deeply phenotyped individuals. Nature Medicine, 1-12.

Bacanl, M., Başaran, N., 2019. Importance of antibiotic residues in animal food. Food and Chemical Toxicology 125, 462-466.

Bajželj, B., Richards, K.S., Allwood, J.M., Smith, P., Dennis, J.S., Curmi, E., Gilligan, C.A., 2014. Importance of food-demand management for climate mitigation. Nature Climate Change $4(10), 924$.

Béné, C., Oosterveer, P., Lamotte, L., Brouwer, I.D., de Haan, S., Prager, S.D., Talsma, E.F., Khoury, C.K., 2019. When food systems meet sustainability-Current narratives and implications for actions. World Development 113, 116-130.

Benoit-Norris, C., Cavan, D.A., Norris, G., 2012. Identifying social impacts in product supply chains: overview and application of the social hotspot database. Sustainability 4(9), 1946-1965.

Benvenuti, L., De Santis, A., Di Sero, A., Franco, N., 2019. Concurrent economic and environmental impacts of food consumption: are low emissions diets affordable? Journal of Cleaner Production 236, 117645.

Beretta, C., Stucki, M., Hellweg, S., 2017. Environmental impacts and hotspots of food losses: value chain analysis of Swiss food consumption. Environmental science \& technology 51(19), 1116511173. 
Beydoun, M.A., 2014. The interplay of gender, mood, and stress hormones in the association between emotional eating and dietary behavior. The Journal of nutrition 144(8), 1139-1141.

Blok, M.C., Spek, J.W., 2016. CVB Feed Table 2016. www.cvbdiervoeding.nl.

Brown, K.A., Timotijevic, L., Barnett, J., Shepherd, R., Lähteenmäki, L., Raats, M.M., 2011. A review of consumer awareness, understanding and use of food-based dietary guidelines. British Journal of Nutrition 106(1), 15-26.

Burlingame, B., Dernini, S., 2012. Sustainable diets and biodiversity directions and solutions for policy, research and action. FAO Headquarters, Rome.

Caldeira, C., De Laurentiis, V., Corrado, S., van Holsteijn, F., Sala, S., 2019. Quantification of food waste per product group along the food supply chain in the European Union: a mass flow analysis. Resources, Conservation and Recycling 149, 479-488.

Campbell, B.M., Beare, D.J., Bennett, E.M., Hall-Spencer, J.M., Ingram, J.S., Jaramillo, F., Ortiz, R., Ramankutty, N., Sayer, J.A., Shindell, D., 2017. Agriculture production as a major driver of the Earth system exceeding planetary boundaries. Ecology and Society 22(4).

Carlson, K.M., Gerber, J.S., Mueller, N.D., Herrero, M., MacDonald, G.K., Brauman, K.A., Havlik, P., O'Connell, C.S., Johnson, J.A., Saatchi, S., 2017. Greenhouse gas emissions intensity of global croplands. Nature Climate Change 7(1), 63-68.

Chalupa-Krebzdak, S., Long, C.J., Bohrer, B.M., 2018. Nutrient density and nutritional value of milk and plant-based milk alternatives. International dairy journal 87, 84-92.

Chatelan, A., Beer-Borst, S., Randriamiharisoa, A., Pasquier, J., Blanco, J., Siegenthaler, S., Paccaud, F., Slimani, N., Nicolas, G., Camenzind-Frey, E., 2017a. Major differences in diet across three linguistic regions of Switzerland: Results from the first national nutrition survey menuCH. Nutrients 9(11), 1163.

Chatelan, A., Beer-Borst, S., Randriamiharisoa, A., Pasquier, J., Blanco, J.M., Siegenthaler, S., Paccaud, F., Slimani, N., Nicolas, G., Camenzind-Frey, E., 2017b. Major differences in diet across three linguistic regions of Switzerland: Results from the first national nutrition survey menuCH. Nutrients 9(11), 1163.

Chen, C., Chaudhary, A., Mathys, A., 2019. Dietary change scenarios and implications for environmental, nutrition, human health and economic dimensions of food sustainability. Nutrients 11(4), 856.

Chiuve, S.E., Fung, T.T., Rimm, E.B., Hu, F.B., McCullough, M.L., Wang, M., Stampfer, M.J., Willett, W.C., 2012. Alternative dietary indices both strongly predict risk of chronic disease. The Journal of nutrition 142(6), 1009-1018.

Clemente, J.C., Ursell, L.K., Parfrey, L.W., Knight, R., 2012. The impact of the gut microbiota on human health: an integrative view. Cell 148(6), 1258-1270.

Cocate, P.G., Natali, A.J., Oliveira, A.d., Alfenas, R.d.C.G., Peluzio, M.d.C.G., Longo, G.Z., Santos, E.C.d., Buthers, J.M., de Oliveira, L.L., Hermsdorff, H.H.M., 2015. Red but not white meat consumption is associated with metabolic syndrome, insulin resistance and lipid peroxidation in Brazilian middle-aged men. European journal of preventive cardiology 22(2), 223-230.

Conlon, M.A., Bird, A.R., 2015. The impact of diet and lifestyle on gut microbiota and human health. Nutrients 7(1), 17-44.

Dahlstrom, M.F., 2014. Using narratives and storytelling to communicate science with nonexpert audiences. Proceedings of the National Academy of Sciences 111(Supplement 4), 13614-13620.

De Boer, I.J., Van Ittersum, M.K., 2018. Circularity in agricultural production. Wageningen University \& Research.

De Olde, E.M., Oudshoorn, F.W., Bokkers, E.A., Stubsgaard, A., Sørensen, C.A., De Boer, I.J., 2016. Assessing the sustainability performance of organic farms in Denmark. Sustainability 8(9), 957. 
De Ponti, T., Rijk, B., Van Ittersum, M.K., 2012. The crop yield gap between organic and conventional agriculture. Agricultural systems 108, 1-9.

Deaton, A., Muellbauer, J., 1980. An almost ideal demand system. The American economic review 70(3), 312-326.

Del Gobbo, L.C., Khatibzadeh, S., Imamura, F., Micha, R., Shi, P., Smith, M., Myers, S.S., Mozaffarian, D., 2015. Assessing global dietary habits: a comparison of national estimates from the FAO and the Global Dietary Database. The American journal of clinical nutrition 101(5), 1038-1046.

Donati, M., Menozzi, D., Zighetti, C., Rosi, A., Zinetti, A., Scazzina, F., 2016. Towards a sustainable diet combining economic, environmental and nutritional objectives. Appetite 106, 48-57.

EFFPA, 2019. EFFPA Figures and Network. Keeping food losses in the food chain.

EFSA, 2017. Dietary Reference Values for nutrients Summary report, in: Publ., E.S. (Ed.).

Elizabeth, L., Machado, P., Zinöcker, M., Baker, P., Lawrence, M., 2020. Ultra-processed foods and health outcomes: a narrative review. Nutrients 12(7), 1955.

Eme, P.E., Douwes, J., Kim, N., Foliaki, S., Burlingame, B., 2019. Review of methodologies for assessing sustainable diets and potential for development of harmonised indicators. International journal of environmental research and public health 16(7), 1184.

Erb, K.-H., Lauk, C., Kastner, T., Mayer, A., Theurl, M.C., Haberl, H., 2016. Exploring the biophysical option space for feeding the world without deforestation. Nature communications 7,11382 .

Ernstoff, A., Stylianou, K.S., Sahakian, M., Godin, L., Dauriat, A., Humbert, S., Erkman, S., Jolliet, O., 2020. Towards win-win policies for healthy and sustainable diets in Switzerland. Nutrients 12(9), 2745.

Etemadi, A., Sinha, R., Ward, M.H., Graubard, B.I., Inoue-Choi, M., Dawsey, S.M., Abnet, C.C., 2017. Mortality from different causes associated with meat, heme iron, nitrates, and nitrites in the NIH-AARP Diet and Health Study: population based cohort study. Bmj 357.

FAO, 1996. Technical Conversion Factors for Agricultural Commodities. Food and Agriculture Organization of the United Nations.

FAO, 2001. FAOSTAT: Food balance sheets. Rome, Italy: Food and Agriculture Organization of the United Nations. Italy.

FAO, 2013. World food and agriculture: Statistical Yearbook. Food and Agriculture Organization of the United Nations, Rome 15.

FAO, 2018. World food and agriculture statistical pocketbook 2018. Food and Agriculture Organization of the United Nations, Rome, Italy.

FAOSTAT, 2018. Agriculture organization corporate statistical database. Accessed on, 25-12.

Feigin, V.L., Roth, G.A., Naghavi, M., Parmar, P., Krishnamurthi, R., Chugh, S., Mensah, G.A., Norrving, B., Shiue, I., Ng, M., 2016. Global burden of stroke and risk factors in 188 countries, during 1990-2013: a systematic analysis for the Global Burden of Disease Study 2013. The Lancet Neurology 15(9), 913-924.

Ferrari, M., Benvenuti, L., Rossi, L., De Santis, A., Sette, S., Martone, D., Piccinelli, R., Le Donne, C., Leclercq, C., Turrini, A., 2020. Could Dietary Goals and Climate Change Mitigation Be Achieved Through Optimized Diet. The Experience of Modeling the National Food Consumption Data in Italy. Front. Nutr. 7: 48. doi: 10.3389/fnut.

Finkbeiner, M., Inaba, A., Tan, R., Christiansen, K., Klüppel, H.-J., 2006. The new international standards for life cycle assessment: ISO 14040 and ISO 14044. The international journal of life cycle assessment 11(2), 80-85. 
Fischer, C.G., Garnett, T., 2016. Plates, pyramids, and planets: developments in national healthy and sustainable dietary guidelines: a state of play assessment. Food and Agriculture Organization of the United Nations.

Fischer, R., Byerlee, D., Edmeades, G., 2014. Crop yields and global food security. ACIAR: Canberra, ACT, 8-11.

Floud, R., Fogel, R.W., Harris, B., Hong, S.C., 2011. The changing body: Health, nutrition, and human development in the western world since 1700. Cambridge University Press.

FOEN, 2019. Switzerland's Greenhouse Gas Inventory 1990-2017, National Inventory Report. Federal Office for the Environment FOEN.

Foley, J.A., Ramankutty, N., Brauman, K.A., Cassidy, E.S., Gerber, J.S., Johnston, M., Mueller, N.D., O'Connell, C., Ray, D.K., West, P.C., 2011. Solutions for a cultivated planet. Nature 478(7369), 337-342.

Frehner, A., Muller, A., Schader, C., De Boer, I.J.M., Van Zanten, H.H.E., 2019. Data for: Methodological choices drive differences in environmentally-friendly dietary solutions.

Frehner, A., Muller, A., Schader, C., De Boer, I.J.M., Van Zanten, H.H.E., 2020. Methodological choices drive differences in environmentally-friendly dietary solutions. Global Food Security 24, 100333.

Frehner, A., Van Zanten, H.H.E., Schader, C., De Boer, I.J.M., Pestoni, G., Rohrmann, S., Muller, A., 2021. How food choices link sociodemographic and lifestyle factors with sustainability impacts. submitted.

Garnett, T., Appleby, M.C., Balmford, A., Bateman, I.J., Benton, T.G., Bloomer, P., Burlingame, B., Dawkins, M., Dolan, L., Fraser, D., 2013. Sustainable intensification in agriculture: premises and policies. Science 341(6141), 33-34.

Garnett, T., Godfray, C., 2012. Sustainable intensification in agriculture. Navigating a course through competing food system priorities. Food climate research network and the Oxford Martin programme on the future of food, University of Oxford, UK 51.

Gazan, R., Brouzes, C.M., Vieux, F., Maillot, M., Lluch, A., Darmon, N., 2018. Mathematical optimization to explore tomorrow's sustainable diets: a narrative review. Advances in Nutrition 9(5), 602-616.

Gemming, L., Mhurchu, C.N., 2016. Dietary under-reporting: what foods and which meals are typically under-reported? European journal of clinical nutrition 70(5), 640-641.

Germani, A., Vitiello, V., Giusti, A.M., Pinto, A., Donini, L.M., del Balzo, V., 2014. Environmental and economic sustainability of the Mediterranean Diet. International journal of food sciences and nutrition 65(8), 1008-1012.

Godfray, H.C.J., Aveyard, P., Garnett, T., Hall, J.W., Key, T.J., Lorimer, J., Pierrehumbert, R.T., Scarborough, P., Springmann, M., Jebb, S.A., 2018. Meat consumption, health, and the environment. Science 361(6399).

Godfray, H.C.J., Beddington, J.R., Crute, I.R., Haddad, L., Lawrence, D., Muir, J.F., Pretty, J., Robinson, S., Thomas, S.M., Toulmin, C., 2010. Food security: the challenge of feeding 9 billion people. science 327(5967), 812-818.

Goldstein, B., Hansen, S.F., Gjerris, M., Laurent, A., Birkved, M., 2016. Ethical aspects of life cycle assessments of diets. Food Policy 59, 139-151.

Gonzalez Fischer, C., Garnett, T., 2016. Plates, pyramids, planet.

Green, M.C., Brock, T.C., 2000. The role of transportation in the persuasiveness of public narratives. Journal of personality and social psychology 79(5), 701.

Haberl, H., Erb, K.H., Krausmann, F., Gaube, V., Bondeau, A., Plutzar, C., Gingrich, S., Lucht, W., Fischer-Kowalski, M., 2007. Quantifying and mapping the human appropriation of net 
primary production in earth's terrestrial ecosystems. Proceedings of the National Academy of Sciences 104(31), 12942-12947.

Hallström, E., Börjesson, P., 2013. Meat-consumption statistics: reliability and discrepancy. Sustainability: Science, Practice and Policy 9(2), 37-47.

Hallström, E., Carlsson-Kanyama, A., Börjesson, P., 2015. Environmental impact of dietary change: a systematic review. Journal of Cleaner Production 91, 1-11.

Hawkes, C., 2008. Dietary implications of supermarket development: a global perspective. Development Policy Review 26(6), 657-692.

Hebinck, A., Zurek, M., Achterbosch, T., Forkman, B., Kuijsten, A., Kuiper, M., Nørrung, B., van't Veer, P., Leip, A., 2020. A sustainability compass for policy navigation to sustainable food systems.

Hedenus, F., Wirsenius, S., Johansson, D.J., 2014. The importance of reduced meat and dairy consumption for meeting stringent climate change targets. Climatic change 124(1-2), 79-91.

Hendrie, G., Ridoutt, B., Wiedmann, T., Noakes, M., 2014. Greenhouse gas emissions and the Australian diet-comparing dietary recommendations with average intakes. Nutrients 6(1), 289-303.

Herforth, A., Arimond, M., Álvarez-Sánchez, C., Coates, J., Christianson, K., Muehlhoff, E., 2019. A global review of food-based dietary guidelines. Advances in Nutrition 10(4), 590-605.

Herrero, M., Thornton, P.K., Mason-D'Croz, D., Palmer, J., Benton, T.G., Bodirsky, B.L., Bogard, J.R., Hall, A., Lee, B., Nyborg, K., 2020. Innovation can accelerate the transition towards a sustainable food system. Nature Food 1(5), 266-272.

Hiza, H.A., Casavale, K.O., Guenther, P.M., Davis, C.A., 2013. Diet quality of Americans differs by age, sex, race/ethnicity, income, and education level. Journal of the Academy of Nutrition and Dietetics 113(2), 297-306.

Hoek, A.C., Malekpour, S., Raven, R., Court, E., Byrne, E., 2021. Towards environmentally sustainable food systems: decision-making factors in sustainable food production and consumption. Sustainable Production and Consumption 26, 610-626.

Huarachi, D.A.R., Piekarski, C.M., Puglieri, F.N., de Francisco, A.C., 2020. Past and future of Social Life Cycle Assessment: Historical evolution and research trends. Journal of Cleaner Production, 121506.

Huber, J., 2000. Towards industrial ecology: sustainable development as a concept of ecological modernization. Journal of environmental policy and planning 2(4), 269-285.

Hyland, J.J., McCarthy, M.B., Henchion, M., McCarthy, S.N., 2017. Dietary emissions patterns and their effect on the overall climatic impact of food consumption. International journal of food science \& technology 52(12), 2505-2512.

IAFFD, 2018. International Aquaculture Feed Formulation Database.

IPCC, 2019. IPCC special report on climate change, desertification, land degradation, sustainable land management, food security, and greenhouse gas fluxes in terrestrial ecosystems : summary for policymakers / IPCC, Intergovernmental Panel on Climate Change, Approved draft ed. IPCC, Place of publication not identified.

IPCC, 2006a. 2006 IPCC guidelines for national greenhouse gas inventories. Institute for Global Environmental Strategies Hayama, Japan.

IPCC, 2006b. Chapter 11: N20 Emissions From Managed Soils, and CO2 Emisions From Lime and Urea application. . IPCC Guidelines for National Greenhouse Gas Inventories.

IPCC, 2019a. Chapter 10: Emissions from livestock and manure management. IPCC guidelines for national greenhouse gas inventories, prepared by the National Greenhouse Gas Inventories Programme, 10.11-10.87. 
IPCC, 2019b. IPCC special report on climate change, desertification, land degradation, sustainable land management, food security, and greenhouse gas fluxes in terrestrial ecosystems : summary for policymakers.

Jones, A.D., Hoey, L., Blesh, J., Miller, L., Green, A., Shapiro, L.F., 2016. A systematic review of the measurement of sustainable diets. Advances in nutrition 7(4), 641-664.

Karlsson, J.O., Parodi, A., Van Zanten, H.H.E., Hansson, P.-A., Röös, E., 2020. Halting European Union soybean feed imports favours ruminants over pigs and poultry. Nature Food, 1-9.

Kastner, T., Kastner, M., Nonhebel, S., 2011. Tracing distant environmental impacts of agricultural products from a consumer perspective. Ecological Economics 70(6), 1032-1040.

Keats, S., Wiggins, S., 2019. Future diets: implications for agriculture and food prices.

Krieger, J.-P., Pestoni, G., Cabaset, S., Brombach, C., Sych, J., Schader, C., Faeh, D., Rohrmann, S., 2019. Dietary patterns and their sociodemographic and lifestyle determinants in Switzerland: Results from the national nutrition survey menuCH. Nutrients 11(1), 62.

Kubik, M.Y., Lytle, L.A., Hannan, P.J., Perry, C.L., Story, M., 2003. The association of the school food environment with dietary behaviors of young adolescents. American journal of public health 93(7), 1168-1173.

Lagerwerf, L., Bannink, A., Van Bruggen, C., Groenestein, C., Huijsmans, J., Van der Kolk, J., Luesink, H., Van der Sluis, S., Velthof, G., Vonk, J., 2019. Methodology for estimating emissions from agriculture in the Netherlands: Calculations of $\mathrm{CH} 4, \mathrm{NH} 3, \mathrm{~N} 2 \mathrm{O}, \mathrm{NOx}$, NMVOC, PM10, PM2. 5 and CO2 with the National Emission Model for Agriculture (NEMA), Update 2019. Statutory Research Tasks Unit for Nature \& the Environment.

Latka, C., Kuiper, M., Frank, S., Heckelei, T., Havlík, P., Witzke, H.-P., Leip, A., Cui, H.D., Kuijsten, A., Geleijnse, J.M., 2021. Paying the price for environmentally sustainable and healthy EU diets. Global Food Security 28, 100437.

Li, H., Wu, T., Wang, X., Qi, Y., 2016. The greenhouse gas footprint of China's food system: an analysis of recent trends and future scenarios. Journal of Industrial Ecology 20(4), 803-817.

Lokuge, M.N., Zivkovic, S., Lange, K., Chidmi, B., 2019. Estimation of a censored food demand system and nutrient elasticities: a cross-sectional analysis of Sri Lanka. International Food and Agribusiness Management Review 22(1030-2019-3343), 717-730.

Lori, M., Symnaczik, S., Mäder, P., De Deyn, G., Gattinger, A., 2017. Organic farming enhances soil microbial abundance and activity - A meta-analysis and meta-regression. PloS one 12(7), e0180442.

Macdiarmid, J.I., Kyle, J., Horgan, G.W., Loe, J., Fyfe, C., Johnstone, A., McNeill, G., 2012. Sustainable diets for the future: can we contribute to reducing greenhouse gas emissions by eating a healthy diet? The American journal of clinical nutrition 96(3), 632-639.

MacLeod, M., Hasan, M.R., Robb, D.H., Mamun-Ur-Rashid, M., 2019. Quantifying and mitigating greenhouse gas emissions from global aquaculture. Food and Agriculture Organization of the United Nations.

Mäder, P., Fliessbach, A., Dubois, D., Gunst, L., Fried, P., Niggli, U., 2002. Soil fertility and biodiversity in organic farming. Science 296(5573), 1694-1697.

Magouras, I., Brookes, V.J., Jori, F., Martin, A., Pfeiffer, D.U., Dürr, S., 2020. Emerging Zoonotic Diseases: Should We Rethink the Animal-Human Interface? Frontiers in Veterinary Science 7, 748.

Mariotti, F., Gardner, C.D., 2019. Dietary protein and amino acids in vegetarian diets-A review. Nutrients 11(11), 2661.

McFadden, J.R., Huffman, W.E., 2017. Willingness-to-pay for natural, organic, and conventional foods: The effects of information and meaningful labels. Food Policy 68, 214-232. 
Meier, M.S., Stoessel, F., Jungbluth, N., Juraske, R., Schader, C., Stolze, M., 2015. Environmental impacts of organic and conventional agricultural products-Are the differences captured by life cycle assessment? Journal of environmental management 149, 193-208.

Meier, T., Christen, O., 2013. Environmental impacts of dietary recommendations and dietary styles: Germany as an example. Environmental science \& technology 47(2), 877-888.

Mertens, E., Kuijsten, A., Van Zanten, H.H.E., Kaptijn, G., Dofková, M., Mistura, L., D'Addezio, L., Turrini, A., Dubuisson, C., Havard, S., 2019. Dietary choices and environmental impact in four European countries. Journal of Cleaner Production 237, 117827.

Meybeck, A., Gitz, V., 2017. Sustainable diets within sustainable food systems. Proceedings of the Nutrition Society 76(1), 1-11.

Michel, F., Hartmann, C., Siegrist, M., 2021. Consumers' associations, perceptions and acceptance of meat and plant-based meat alternatives. Food Quality and Preference 87, 104063.

Milner, J., Joy, E.J., Green, R., Harris, F., Aleksandrowicz, L., Agrawal, S., Smith, P., Haines, A., Dangour, A.D., 2017. Projected health effects of realistic dietary changes to address freshwater constraints in India: a modelling study. The Lancet Planetary Health 1(1), e26-e32.

Moher, D., Liberati, A., Tetzlaff, J., Altman, D.G., 2009. Preferred reporting items for systematic reviews and meta-analyses: the PRISMA statement. Annals of internal medicine 151(4), 264269.

Mohr, M., Schlich, M., 2016. Socio-demographic basic factors of G erman customers as predictors for sustainable consumerism regarding foodstuffs and meat products. International Journal of Consumer Studies 40(2), 158-167.

Moschitz, H., Oehen, B., 2020. Creating value (s) by integrating local and extra-local resources in cereal production in the Swiss Alps. The International Journal of Sociology of Agriculture and Food 26(1), 48-68.

Mottet, A., De Haan, C., Falcucci, A., Tempio, G., Opio, C., Gerber, P., 2017. Livestock: On our plates or eating at our table? A new analysis of the feed/food debate. Global Food Security 14, $1-8$.

Mozaffarian, D., Angell, S.Y., Lang, T., Rivera, J.A., 2018. Role of government policy in nutritionbarriers to and opportunities for healthier eating. Bmj 361.

Muller, A., Frehner, A., Pfeiffer, C., Moakes, S., Schader, C., 2020. SOLm Model Documentation, updated version from Deliverable 4.1 from the EU-UNISECO project.

https://orgprints.org/38778/.

Muller, A., Schader, C., Scialabba, N.E.-H., Brüggemann, J., Isensee, A., Erb, K.-H., Smith, P., Klocke, P., Leiber, F., Stolze, M., 2017. Strategies for feeding the world more sustainably with organic agriculture. Nature communications 8(1), 1-13.

Murphy, S.P., Allen, L.H., 2003. Nutritional importance of animal source foods. The Journal of nutrition 133(11), 3932S-3935S.

Muscat, A., De Olde, E., De Boer, I.J.M., Ripoll-Bosch, R., 2019. The battle for biomass: A systematic review of food-feed-fuel competition. Global Food Security, 100330.

Nathani, C., Schmid, C., Van Nieuwkoop, R., 2011. Schätzung einer Input-Output-Tabelle der Schweiz 2008. Bundesamt für Statistik, Neuchâtel.

NEVO-table, N., 2019. Dutch food composition table. Zeist: NEVO foundation (in Dutch).

Niederdeppe, J., Connelly, N.A., Lauber, T.B., Knuth, B.A., 2019. Effects of a personal narrative in messages designed to promote healthy fish consumption among women of childbearing age. Health communication 34(8), 825-837. 
Norris, C.B., Norris, G.A., Aulisio, D., 2014. Efficient assessment of social hotspots in the supply chains of 100 product categories using the social hotspots database. Sustainability 6(10), 69736984.

Nosworthy, M.G., Neufeld, J., Frohlich, P., Young, G., Malcolmson, L., House, J.D., 2017. Determination of the protein quality of cooked Canadian pulses. Food science \& nutrition 5(4), 896-903.

Notarnicola, B., Sala, S., Anton, A., McLaren, S.J., Saouter, E., Sonesson, U., 2017. The role of life cycle assessment in supporting sustainable agri-food systems: A review of the challenges. Journal of Cleaner Production 140, 399-409.

O'brien, R.M., 2007. A caution regarding rules of thumb for variance inflation factors. Quality \& quantity 41(5), 673-690.

Organization, W.H., 2017. Global action plan for the prevention and control of noncommunicable diseases 2013-2020.[Internet]. 2013.

Parodi, A., Leip, A., De Boer, I., Slegers, P., Ziegler, F., Temme, E.H., Herrero, M., Tuomisto, H., Valin, H., Van Middelaar, C., Van Loon, J.J.A., Van Zanten, H.H.E., 2018. The potential of future foods for sustainable and healthy diets. Nature Sustainability 1(12), 782-789.

Pasquier, J., Chatelan, A., Bochud, M., 2017. Weighting Strategy. April 2017. Available online: https://menuch.iumsp.ch/index.php/catalog/4/download/17 (accessed on 24 July 2018).

Persson, U.M., Johansson, D.J., Cederberg, C., Hedenus, F., Bryngelsson, D., 2015. Climate metrics and the carbon footprint of livestock products: where's the beef? Environmental Research Letters 10(3), 034005.

Pestoni, G., Krieger, J.-P., Sych, J.M., Faeh, D., Rohrmann, S., 2019. Cultural differences in diet and determinants of diet quality in Switzerland: Results from the National Nutrition Survey MenuCH. Nutrients 11(1), 126.

Plutzar, C., Kroisleitner, C., Haberl, H., Fetzel, T., Bulgheroni, C., Beringer, T., Hostert, P., Kastner, T., Kuemmerle, T., Lauk, C., 2016. Changes in the spatial patterns of human appropriation of net primary production (HANPP) in Europe 1990-2006. Regional Environmental Change 16(5), 1225-1238.

Ponisio, L.C., M'Gonigle, L.K., Mace, K.C., Palomino, J., De Valpine, P., Kremen, C., 2015.

Diversification practices reduce organic to conventional yield gap. Proceedings of the Royal Society B: Biological Sciences 282(1799), 20141396.

Poore, J., Nemecek, T., 2018. Reducing food's environmental impacts through producers and consumers. Science 360(6392), 987-992.

Post, M.J., Levenberg, S., Kaplan, D.L., Genovese, N., Fu, J., Bryant, C.J., Negowetti, N., Verzijden, K., Moutsatsou, P., 2020. Scientific, sustainability and regulatory challenges of cultured meat. Nature Food 1(7), 403-415.

Pretty, J., Benton, T.G., Bharucha, Z.P., Dicks, L.V., Flora, C.B., Godfray, H.C.J., Goulson, D., Hartley, S., Lampkin, N., Morris, C., 2018. Global assessment of agricultural system redesign for sustainable intensification. Nature Sustainability 1(8), 441-446.

Proulx, A.K., Reddy, M.B., 2007. Fermentation and lactic acid addition enhance iron bioavailability of maize. Journal of agricultural and food chemistry 55(7), 2749-2754.

Ridoutt, B.G., Hendrie, G.A., Noakes, M., 2017. Dietary strategies to reduce environmental impact: a critical review of the evidence base. Advances in Nutrition 8(6), 933-946.

Rijk, B., van Ittersum, M., Withagen, J., 2013. Genetic progress in Dutch crop yields. Field Crops Research 149, 262-268. 
Rohmer, S., Gerdessen, J.C., Claassen, G., 2019. Sustainable supply chain design in the food system with dietary considerations: A multi-objective analysis. European Journal of Operational Research 273(3), 1149-1164.

Röös, E., Bajželj, B., Smith, P., Patel, M., Little, D., Garnett, T., 2017. Greedy or needy? Land use and climate impacts of food in 2050 under different livestock futures. Global Environmental Change 47, 1-12.

Röös, E., Patel, M., Spångberg, J., Carlsson, G., Rydhmer, L., 2016. Limiting livestock production to pasture and by-products in a search for sustainable diets. Food Policy 58, 1-13.

Sabate, J., Soret, S., 2014. Sustainability of plant-based diets: back to the future. The American journal of clinical nutrition, 476S-482S.

Saxe, H., 2014. The New Nordic Diet is an effective tool in environmental protection: it reduces the associated socioeconomic cost of diets. The American journal of clinical nutrition 99(5), 11171125.

Scarborough, P., Appleby, P.N., Mizdrak, A., Briggs, A.D., Travis, R.C., Bradbury, K.E., Key, T.J., 2014. Dietary greenhouse gas emissions of meat-eaters, fish-eaters, vegetarians and vegans in the UK. Climatic change 125(2), 179-192.

Schader, C., Muller, A., El-Hage Scialabba, N., Hecht, J., Stolze, M., 2014. Comparing global and product-based LCA perspectives on environmental impacts of low-concentrate ruminant production, Proc. 9th Int. Conf. on Life Cycle Assessment in the Agri-Food Sector, San Francisco, CA, 8. pp. 1203-1209.

Schader, C., Muller, A., Scialabba, N.E.-H., Hecht, J., Isensee, A., Erb, K.-H., Smith, P., Makkar, H.P., Klocke, P., Leiber, F., 2015. Impacts of feeding less food-competing feedstuffs to livestock on global food system sustainability. Journal of The Royal Society Interface 12(113), 20150891.

Schwingshackl, L., Bogensberger, B., Hoffmann, G., 2018. Diet quality as assessed by the healthy eating index, alternate healthy eating index, dietary approaches to stop hypertension score, and health outcomes: an updated systematic review and meta-analysis of cohort studies. Journal of the Academy of Nutrition and Dietetics 118(1), 74-100. e111.

Schwingshackl, L., Watzl, B., Meerpohl, J.J., 2020. The healthiness and sustainability of food based dietary guidelines. British Medical Journal Publishing Group.

Senghor, B., Sokhna, C., Ruimy, R., Lagier, J.-C., 2018. Gut microbiota diversity according to dietary habits and geographical provenance. Human Microbiome Journal 7, 1-9.

Seufert, V., 2019. Comparing yields: Organic versus conventional agriculture, Encyclopedia of Food Security and Sustainability: Volume 3: Sustainable Food Systems and Agriculture. Elsevier, pp. 196-208.

Seufert, V., Ramankutty, N., 2017. Many shades of gray-The context-dependent performance of organic agriculture. Science advances 3(3), e1602638.

Seufert, V., Ramankutty, N., Foley, J.A., 2012. Comparing the yields of organic and conventional agriculture. Nature 485(7397), 229-232.

Seufert, V., Ramankutty, N., Mayerhofer, T., 2017. What is this thing called organic?-How organic farming is codified in regulations. Food Policy 68, 10-20.

SGE, 2020. Nachhaltiger geniessen - so kann's gehen! https://www.sge-ssn.ch/comic/.

Shepherd, R., 2005. Influences on food choice and dietary behavior. Diet diversification and health promotion 57, 36-43.

Shimazaki, T., Matsushita, M., Iio, M., Takenaka, K., 2018. Use of health promotion manga to encourage physical activity and healthy eating in Japanese patients with metabolic syndrome: a case study. Archives of Public Health 76(1), 1-9. 
Smil, V., 2002. Eating meat: evolution, patterns, and consequences. Population and development review 28(4), 599-639.

SNF, 2020. Healthy Nutrition and Sustainable Food Production.

http://www.healthyandsustainable.ch/en/simulator.

Snyder, L.B., 2007. Health communication campaigns and their impact on behavior. Journal of nutrition education and behavior 39(2), S32-S40.

Song, G., Gao, X., Fullana-i-Palmer, P., Lv, D., Zhu, Z., Wang, Y., Bayer, L.B., 2019. Shift from feeding to sustainably nourishing urban China: A crossing-disciplinary methodology for global environment-food-health nexus. Science of The Total Environment 647, 716-724.

Springmann, M., Clark, M., Mason-D'Croz, D., Wiebe, K., Bodirsky, B.L., Lassaletta, L., De Vries, W., Vermeulen, S.J., Herrero, M., Carlson, K.M., 2018a. Options for keeping the food system within environmental limits. Nature 562(7728), 519-525.

Springmann, M., Spajic, L., Clark, M.A., Poore, J., Herforth, A., Webb, P., Rayner, M., Scarborough, P., 2020. The healthiness and sustainability of national and global food based dietary guidelines: modelling study. bmj 370.

Springmann, M., Wiebe, K., Mason-D'Croz, D., Sulser, T.B., Rayner, M., Scarborough, P., $2018 b$. Health and nutritional aspects of sustainable diet strategies and their association with environmental impacts: a global modelling analysis with country-level detail. The Lancet Planetary Health 2(10), e451-e461.

Steffen, W., Richardson, K., Rockström, J., Cornell, S.E., Fetzer, I., Bennett, E.M., Biggs, R., Carpenter, S.R., De Vries, W., De Wit, C.A., 2015. Planetary boundaries: Guiding human development on a changing planet. Science 347(6223).

Stocker, T., 2014. Climate change 2013: the physical science basis: Working Group I contribution to the Fifth assessment report of the Intergovernmental Panel on Climate Change. Cambridge university press.

Stolze, M., Schader, C., Muller, A., Frehner, A., Kopainsky, B., Nathani, C., Brandes, J., Rohrmann, S., Brombach, C., Krieger, J.-P., 2019a. Sustainable and healthy diets: trade-offs and synergies: final scientific report.

Stolze, M., Weisshaidinger, R., Bartel, A., Schwank, O., Müller, A., Biedermann, R., 2019b. Chancen der Landwirtschaft in den Alpenländern-Wege zu einer raufutterbasierten Milch-und Fleischproduktion in Österreich und der Schweiz. Haupt Verlag.

Szczebyło, A., Rejman, K., Halicka, E., Laskowski, W., 2020. Towards More Sustainable DietsAttitudes, Opportunities and Barriers to Fostering Pulse Consumption in Polish Cities. Nutrients 12(6), 1589.

Talsma, E.F., Melse-Boonstra, A., Brouwer, I.D., 2017. Acceptance and adoption of biofortified crops in low-and middle-income countries: a systematic review. Nutrition reviews 75(10), 798829.

Thaler, S., Zessner, M., Weigl, M., Rechberger, H., Schilling, K., Kroiss, H., 2015. Possible implications of dietary changes on nutrient fluxes, environment and land use in Austria. Agricultural Systems 136, 14-29.

Theurl, M.C., Lauk, C., Kalt, G., Mayer, A., Kaltenegger, K., Morais, T.G., Teixeira, R.F., Domingos, T., Winiwarter, W., Erb, K.-H., 2020. Food systems in a zero-deforestation world: Dietary change is more important than intensification for climate targets in 2050. Science of The Total Environment 735, 139353.

Thornton, P.K., 2010. Livestock production: recent trends, future prospects. Philosophical Transactions of the Royal Society B: Biological Sciences 365(1554), 2853-2867. 
Tilman, D., Clark, M., 2014. Global diets link environmental sustainability and human health. Nature 515(7528), 518-522.

Tuomisto, H.L., 2018. Importance of considering environmental sustainability in dietary guidelines. The Lancet Planetary Health 2(8), e331-e332.

Tuomisto, H.L., 2019. The eco-friendly burger: could cultured meat improve the environmental sustainability of meat products? EMBO reports 20(1), e47395.

Van De Kamp, M.E., Van Dooren, C., Hollander, A., Geurts, M., Brink, E.J., Van Rossum, C., Biesbroek, S., De Valk, E., Toxopeus, I.B., Temme, E.H., 2018. Healthy diets with reduced environmental impact?-The greenhouse gas emissions of various diets adhering to the Dutch food based dietary guidelines. Food Research International 104, 14-24.

Van der Weele, C., Feindt, P., Van Der Goot, A.J., Van Mierlo, B., Van Boekel, M., 2019. Meat alternatives: an integrative comparison. Trends in Food Science \& Technology 88, 505-512.

Van Der Werf, H.M., Knudsen, M.T., Cederberg, C., 2020. Towards better representation of organic agriculture in life cycle assessment. Nature Sustainability 3(6), 419-425.

Van Hal, O., 2020. Upcycling biomass in a circular food system: The role of livestock and fish. Wageningen University.

Van Hal, O., De Boer, I.J.M., Muller, A., De Vries, S., Erb, K.-H., Schader, C., Gerrits, W., Van Zanten, H.H.E., 2019a. Upcycling food leftovers and grass resources through livestock: impact of livestock system and productivity. Journal of Cleaner Production 219, 485-496.

Van Hal, O., Weijenberg, A., De Boer, I.J.M., Van Zanten, H.H.E., 2019b. Accounting for feed-food competition in environmental impact assessment: Towards a resource efficient food-system. Journal of Cleaner Production 240, 118241.

Van Kernebeek, H., Oosting, S., Feskens, E., Gerber, P., De Boer, I., 2014. The effect of nutritional quality on comparing environmental impacts of human diets. Journal of cleaner production 73, 88-99.

Van Kernebeek, H.R., Oosting, S.J., Van Ittersum, M.K., Bikker, P., De Boer, I.J., 2016. Saving land to feed a growing population: consequences for consumption of crop and livestock products. The International Journal of Life Cycle Assessment 21(5), 677-687.

Van Selm, B., Frehner, A., De Boer, I.J.M., Van Hal, O., Hijbeek, R., Van Ittersum, M., Talsma, E., Lesschen, J.-P., Hendriks, C., Herrero, M., Van Zanten, H.H.E., 2021. The compatibility of animal-sourced food and circularity in healthy European diets.

Van Zanten, H.H.E., Herrero, M., Van Hal, O., Röös, E., Muller, A., Garnett, T., Gerber, P.J., Schader, C., De Boer, I.J.M., 2018. Defining a land boundary for sustainable livestock consumption. Global change biology.

Van Zanten, H.H.E., Mollenhorst, H., Klootwijk, C.W., Van Middelaar, C.E., De Boer, I.J.M., 2016. Global food supply: land use efficiency of livestock systems. The International Journal of Life Cycle Assessment 21(5), 747-758.

Van Zanten, H.H.E., Van Ittersum, M.K., De Boer, I.J.M., 2019. The role of farm animals in a circular food system. Global Food Security 21, 18-22.

Vassallo, M., Scalvedi, M.L., Saba, A., 2016. Investigating psychosocial determinants in influencing sustainable food consumption in Italy. International Journal of Consumer Studies 40(4), 422434.

Vellinga, T.V., Blonk, H., Marinussen, M., Van Zeist, W., Starmans, D., 2013. Methodology used in feedprint: a tool quantifying greenhouse gas emissions of feed production and utilization. Wageningen UR Livestock Research. 
Velthof, G., Oudendag, D., Witzke, H., Asman, W., Klimont, Z., Oenema, O., 2009. Integrated assessment of nitrogen losses from agriculture in EU-27 using MITERRA-EUROPE. Journal of Environmental Quality 38(2), 402-417.

Verbeke, W.A., Viaene, J., 2000. Ethical challenges for livestock production: Meeting consumer concerns about meat safety and animalwelfare. Journal of Agricultural and Environmental Ethics 12(2), 141-151.

Vieux, F., Darmon, N., Touazi, D., Soler, L.G., 2012. Greenhouse gas emissions of self-selected individual diets in France: changing the diet structure or consuming less? Ecological economics 75, 91-101.

Von Ow, A., Waldvogel, T., Nemecek, T., 2020. Environmental optimization of the Swiss population's diet using domestic production resources. Journal of Cleaner Production 248, 119241.

Waijers, P.M., Feskens, E.J., Ocké, M.C., 2007. A critical review of predefined diet quality scores. British journal of nutrition 97(2), 219-231.

Walker, C., Gibney, E.R., Hellweg, S., 2018. Comparison of environmental impact and nutritional quality among a European sample population-findings from the Food4Me study. Scientific reports $8(1), 1-10$.

Walter, P., Infanger, E., Mühlemann, P., 2007. Food pyramid of the Swiss Society for Nutrition. Annals of Nutrition and Metabolism 51(Suppl. 2), 15-20.

WHO, 2007. Protein and amino acid requirements in human nutrition. World Health Organization technical report series(935), 1.

$\mathrm{WHO}, 2018$. The state of food security and nutrition in the world 2018: building climate resilience for food security and nutrition. Food \& Agriculture Org.

WHO, 2019. Healthy diet. World Health Organization. Regional Office for the Eastern Mediterranean.

Wilkinson, J., Lee, M., 2018. Use of human-edible animal feeds by ruminant livestock. Animal 12(8), 1735-1743.

Willett, W., Rockström, J., Loken, B., Springmann, M., Lang, T., Vermeulen, S., Garnett, T., Tilman, D., DeClerck, F., Wood, A., 2019. Food in the Anthropocene: the EAT-Lancet Commission on healthy diets from sustainable food systems. The Lancet 393(10170), 447-492.

Yang, Y., Hobbs, J.E., 2020. The power of stories: Narratives and information framing effects in science communication. American Journal of Agricultural Economics 102(4), 1271-1296.

Zabel, F., Putzenlechner, B., Mauser, W., 2014. Global agricultural land resources-a high resolution suitability evaluation and its perspectives until 2100 under climate change conditions. PloS one 9(9), e107522.

Zom, R., Groenestein, C., 2015. TC-O_20 Excretion of volatile solids by livestock to calculate methane production from manure. RAMIRAN 2015.

Zu Ermgassen, E.K., Phalan, B., Green, R.E., Balmford, A., 2016. Reducing the land use of EU pork production: where there's swill, there'sa way. Food policy 58, 35-48. 


\section{Summary}

Food systems face the challenge of nourishing a growing population while respecting the planetary boundaries. Food production causes substantial adverse environmental impacts, by affecting biogeochemical flows and biosphere integrity as well as by contributing to climate change, landsystem change, and freshwater use. Moreover, the production of animal-source foods (ASF) results in competition for natural resources. Currently, land and biomass that could be used for producing food for direct human food consumption are allocated to feed farm animals instead, leading to substantial resource use inefficiencies in current food systems. Further, food production raises social risks along the food value chain. On the consumption side, dietary habits are an important risk factor for multiple non-communicable diseases, such as diabetes, cardiovascular disease, stroke, and cancer.

In high-income countries, shifting food consumption towards plant-based diets is an often-proposed strategy to simultaneously solving multiple of the stated sustainability challenges. Next to consumption-side approaches, also different production-side strategies as well as overarching solutions, such as avoiding feed-food competition and related transitions towards more circular food systems, are proposed. In all these strategies, ASF play a key role. While ASF contribute to meet human nutritional requirements, they also cause large parts of the sustainability impacts of current food systems - therefore, a balance is needed. To this end, this thesis aimed to investigate the role of ASF in current and future food systems, with special emphasis on different sustainability impacts and by employing different geographical, temporal, and demographic scopes.

While scientific evidence that ASF consumption in high-income countries needs to be reduced is undisputed, dietary solutions differ with regard to the proposed degree of reduction for different ASF. In Chapter 2, we reviewed scientific literature that assessed environmental consequences of different dietary solutions, and identified methodological choices in the environmental impact assessment that can lead to these different solutions. We found that on the one hand, studies employing fixed impact assessments and consumption-oriented scenario specification rather recommend to reduce beef and pork than chicken. In these studies, the role of ASF is mostly limited to current production practices and feed-food competition is not addressed. On the other hand, studies considering systemic consequences and specifying resource-oriented scenarios propose larger reductions of chicken and pork, because these are produced with more food-competing feed. In these studies, the role of animals and the resulting ASF is mostly limited to converting lowopportunity-cost biomass (LCB), thereby avoiding feed-food competition. In future research and communication of more sustainable dietary solutions, these choices and the resulting consequences should be considered, to allow for effective communication and policy design.

Further, in Chapter 3, we assessed multiple sustainability impacts of current diets in Switzerland, and linked these to sociodemographic and lifestyle factors. For this, we employed the dietary recall dataset menuCH. Of these dietary recalls, we calculated two environmental indicators (greenhouse gas emissions and land use), two social measures (social risks by the Social Hotspots Index and diet quality by the Alternate Healthy Eating Index), and diet cost. By means of multiple linear regression, 
we investigated the associations between sociodemographic and lifestyle factors and food choices as well as between sociodemographic and lifestyle factors and sustainability impacts. By qualitatively linking results of these regressions, we were able to reveal how food choices link sociodemographic and lifestyle factors with sustainability impacts. Our results showed that while nationalities, language regions, age groups, and smoking status seemed particularly distinctive, income or educational groups seemed hardly relevant. Food choices and the resulting sustainability impacts thus markedly differed between these factors, suggesting that these can be relevant, for example, to target nutrition campaigns.

Moving from current diets to dietary improvements, we assessed different consumer strategies towards a more sustainable Swiss food system in Chapter 4. These strategies included dietary change, in particular following a vegetarian diet and adhering to the Swiss dietary guidelines, and behaviour changes that target the way the chosen products were produced. For this, we considered increased share of organic produce - also in combination with the circularity principle of feeding only LCB - as well as increased share of domestic produce. Moreover, reducing food waste at consumption stage was included as an additional strategy. Of these strategies, we assessed three environmental impacts, i.e. greenhouse gas emissions, land use, and nitrogen surplus, two social impacts, i.e. social risks (Social Hotspots Index) and diet quality (Alternate Healthy Eating Index), as well as dietary cost. While the dietary changes towards more plant-based diets improved the environmental performance, diet quality, and diet cost, these changes increased social risks. Moreover, increased share of organic agriculture had ambivalent effects on the environmental performance. When enhanced with circularity principles, however, all environmental indicators improved substantially. While these strategies revealed a large mitigation potential for adverse environmental impacts, the strategies of increased domestic produce and reduced food waste had desirable effects as well, but at lower orders of magnitude.

Based on the strategies of adhering to dietary guidelines and implementing circularity principles, we studied the compatibility of ASF recommendations in national dietary guidelines and circularity principles (Chapter 5). We selected five countries that represent different regions of, and settings in, Europe (Bulgaria, Malta, the Netherlands, Sweden, and Switzerland). For each of these countries, we investigated whether ASF recommendations could be met with nationally available LCB only. We employed a resource allocation model that allocates LCB to different animal production systems in order to maximise different nutritional outcomes for humans. The nutritional outcomes were selected such that different (groups of) nutrients were maximised - i.e. protein, fatty acids, minerals, and vitamins. Then, we calculated greenhouse gas emissions and land use of the different optimisation scenarios. We found that all countries recommend substantial levels of ASF, which could not be met with LCB only. Maximum reachable protein contents with LCB ranged from 15.9 $\mathrm{g}$ protein per capita per day (Malta) to $38.9 \mathrm{~g}$ protein per capita per day (Switzerland). The composition of the ASF based on LCB differed substantially from the composition of the ASF recommended in the guidelines - but could be adjusted according to the selected nutritional focus. In sum, we found that limiting ASF to LCB would result in a more circular and climate-friendly food system, requiring revised and lowered ASF recommendations. 
At a broader level than national dietary guidelines, the EAT-Lancet commission recently issued a healthy reference diet that respects planetary boundaries. We investigated the compatibility of ASF in this healthy reference diet with circularity principles at European Union EU-28 level (Chapter 6). Compared to the national dietary guidelines assessed, the EAT-Lancet dietary guidelines recommend substantially lower amounts of ASF. Due to this fact, the overall quantity of ASF protein could be met, although also there, the exact composition could not be met. In particular, the EATLancet dietary guidelines include larger quantities of chicken than beef and pork, while with the available LCB, mainly milk, beef, and pork could be produced. As for the national dietary guidelines assessed, we found that both greenhouse gas emissions and land use could be substantially reduced. Altogether, in order to achieve aligned healthy diets from more circular food systems, the composition of ASF and the permissible substitutability need to be carefully considered.

In Chapter 7, the contribution of the presented findings to a more nuanced debate on the role of ASF in more sustainable food systems was discussed. Our results clearly showed that solutions for more sustainable food systems depend on the sustainability dimensions and the specific indicators included. Therefore, results for strategies towards more sustainable food systems need to be interpreted in the context of the chosen indicators, and the related assumptions, foci, and omissions need to be critically discussed. Further, while substituting ASF with plant-based alternatives revealed many synergies for the environment and human health, we also found trade-offs due to increased social risks. This finding suggests that dietary change needs to be accompanied by suitable measures that address these potential risks. Moreover, to circumvent compatibility issues of integrating ASF recommendations from dietary guidelines with circularity principles, the nutritional function of ASF needs to be clarified. Lastly, the discussion on the implications of the different scopes employed in this thesis revealed that the chosen scope determines the requirements for change towards more sustainable food systems, and thereby also the potential role of ASF therein. In conclusion, the role of ASF in more sustainable food systems can be manifold - and depends largely on the required nutritional contributions as well as on defined priorities for different dimensions of sustainability. 


\section{Zusammenfassung}

Ernährungssysteme stehen vor der Herausforderung, eine wachsende Bevölkerung zu ernähren und gleichzeitig die planetaren Grenzen zu respektieren. Die Nahrungsmittelproduktion verursacht erhebliche negative Umweltauswirkungen, indem sie die biogeochemischen Flüsse und die Integrität der Biosphäre beeinträchtigt sowie zum Klimawandel, zur Veränderung der Landsysteme und zum Süsswasserverbrauch beiträgt. Darüber hinaus führt die Produktion von Nahrungsmitteln tierischer Herkunft (NTH) zu einer Konkurrenz um natürliche Ressourcen. Derzeit werden Land und Biomasse, die für die Produktion von Nahrungsmitteln für den direkten menschlichen Verzehr genutzt werden könnten, stattdessen für die Fütterung von Nutztieren verwendet. Dies führt zu erheblichen Ineffizienzen bei der Ressourcennutzung in den derzeitigen Ernährungssystemen. Die Nahrungsmittelproduktion birgt zudem soziale Risiken entlang der Wertschöpfungskette der Nahrungsmittel. Auf der Seite des Konsums sind die Ernährungsgewohnheiten ein wichtiger Risikofaktor für mehrere nicht übertragbare Krankheiten wie Diabetes, Herz-KreislaufErkrankungen und Krebs.

In Ländern mit hohem Einkommen ist die Umstellung des Lebensmittelkonsums auf eine mehrheitlich pflanzliche Ernährung eine oft vorgeschlagene Strategie, um mehrere der genannten Nachhaltigkeitsherausforderungen gleichzeitig zu lösen. Neben verbrauchsseitigen Ansätzen werden auch verschiedene produktionsseitige Strategien sowie übergreifende Lösungen vorgeschlagen, wie z. B. die Vermeidung von Nahrungsmittel- und Flächenkonkurrenz von Futtermitteln und damit verbundene Übergänge zu stärker zirkulär ausgerichteten Ernährungssystemen. In all diesen Strategien spielen NTH eine Schlüsselrolle. Während NTH dazu beitragen, die Ernährungsbedürfnisse der Menschen zu erfüllen, verursachen sie auch einen grossen Teil der Nachhaltigkeitsauswirkungen der derzeitigen Ernährungssysteme - daher ist ein Gleichgewicht erforderlich. Diese Arbeit zielt darauf ab, die Rolle von NTH in aktuellen und zukünftigen Ernährungssystemen zu untersuchen, mit besonderem Schwerpunkt auf verschiedene Nachhaltigkeitsauswirkungen und unter Betrachtung verschiedener geografischer, zeitlicher und demografischer Horizonte.

Während der wissenschaftliche Nachweis unbestritten ist, dass der Verzehr von NTH in Ländern mit hohem Einkommen reduziert werden muss, unterscheiden sich Lösungen hinsichtlich der vorgeschlagenen Reduktion für verschiedene NTH. In Kapitel 2 wird eine Literaturübersicht präsentiert zur Bewertung der Umweltfolgen verschiedener Ernährungslösungen und deren spezifischen methodischen Entscheidungen. Wir fanden heraus, dass Studien, die fixe Folgenabschätzungen und verbrauchsorientierte Szenariospezifikationen verwenden, eher eine Reduzierung von Rind- und Schweinefleisch als von Geflügelfleisch empfehlen. In diesen Studien ist die Rolle der NTH meist auf die aktuellen Produktionspraktiken beschränkt und die Nahrungsmittel- und Flächenkonkurrenz von Futtermitteln wird nicht berücksichtigt. Zum anderen schlagen Studien, die systemische Konsequenzen berücksichtigen und ressourcenorientierte Szenarien spezifizieren, grössere Reduktionen bei Geflügel- und Schweinefleisch vor, da diese mit mehr Nahrungsmittel- und Flächenkonkurrenz von Futtermitteln produziert werden. In diesen Studien beschränkt sich die Rolle der Nutztiere und der daraus resultierenden NTH meist auf die 
Umwandlung von Biomasse mit niedrigen Opportunitätskosten (BNO), wodurch die Nahrungsmittel- und Flächenkonkurrenz von Futtermitteln vermieden wird. In der zukünftigen Forschung und Kommunikation von nachhaltigeren Ernährungslösungen sollten diese Entscheidungen und die daraus resultierenden Konsequenzen berücksichtigt werden, um eine effektive Kommunikation und Politikgestaltung zu ermöglichen.

Des Weiteren haben wir in Kapitel 3 verschiedene Nachhaltigkeitsauswirkungen der aktuellen Ernährung in der Schweiz bewertet und diese mit soziodemografischen und Lebensstilfaktoren verknüpft. Dazu verwendeten wir die Daten der nationalen Ernährungserhebung menuCH. Aus diesen Daten berechneten wir zwei Umweltindikatoren (Treibhausgasemissionen und Landnutzung), zwei soziale Indikatoren (soziale Risiken durch den Social Hotspots Index und Ernährungsqualität durch den Alternate Healthy Eating Index) sowie die Kosten der jeweiligen Ernährungsweise. Mittels multipler linearer Regression untersuchten wir die Assoziationen zwischen soziodemografischen und Lebensstilfaktoren und der Lebensmittelauswahl sowie zwischen soziodemografischen und Lebensstilfaktoren und Nachhaltigkeitsauswirkungen. Durch die qualitative Verknüpfung der Ergebnisse dieser Regressionen konnten wir aufzeigen, wie die Lebensmittelauswahl mit soziodemografischen und Lebensstilfaktoren und Nachhaltigkeitsauswirkungen zusammenhängt. Unsere Ergebnisse zeigten, dass Unterschiede zwischen Nationalitäten, Sprachregionen, Altersgruppen und Raucherstatus besonders ausgeprägt zu sein scheinen, wohingegen verschiedene Einkommens- oder Bildungsgruppen kaum relevant waren. Die Lebensmittelauswahl und die daraus resultierenden Nachhaltigkeitsauswirkungen unterschieden sich also deutlich zwischen diesen genannten Faktoren, was darauf hindeutet, dass diese beispielsweise für gezielte Ernährungskampagnen relevant sein können.

In Kapitel 4 haben wir verschiedene Strategien der Verbraucher für ein nachhaltigeres Schweizer Ernährungssystem untersucht. Diese Strategien umfassten eine Ernährungsumstellung, insbesondere eine vegetarische Ernährung und die Einhaltung der Schweizer Ernährungsrichtlinien, sowie Verhaltensänderungen, die auf die Art der Herstellung der gewählten Produkte abzielen. Dabei wurde ein erhöhter Anteil an Bioprodukten - auch in Kombination mit dem Kreislaufprinzip der ausschliesslichen Verfütterung von BNO - sowie ein erhöhter Anteil an einheimischen Produkten berücksichtigt. Darüber hinaus wurde die Verringerung der Lebensmittelverschwendung auf der Verbraucherstufe als zusätzliche Strategie einbezogen. Von diesen Strategien bewerteten wir drei Umweltauswirkungen, d.h. Treibhausgasemissionen, Landnutzung und Stickstoffüberschuss, zwei soziale Auswirkungen, d.h. soziale Risiken (Social Hotspots Index) und Ernährungsqualität (Alternate Healthy Eating Index), sowie die Kosten der Ernährung. Während die Ernährungsumstellung auf mehr pflanzliche Kost die Umweltbilanz, die Qualität der Ernährung und die Kosten der Ernährung verbesserte, erhöhten diese Veränderungen die sozialen Risiken. Zudem hatte ein erhöhter Anteil an Bioprodukten ambivalente Auswirkungen auf die Umweltbilanz. Wenn jedoch zusätzlich die Prinzipien der Kreislaufwirtschaft berücksichtigt wurden, verbesserten sich alle Umweltindikatoren erheblich. Während diese Strategien ein grosses Mitigationspotenzial für negative Umwelteinflüsse aufzeigten, hatten die Strategien der vermehrten einheimischen Produktion und der reduzierten Lebensmittelverschwendung ebenfalls wünschenswerte Effekte, jedoch in niedrigeren Grössenordnungen. 
Basierend auf den Strategien der Einhaltung von Ernährungsrichtlinien und der Umsetzung von Kreislaufwirtschaftsprinzipien untersuchten wir die Kompatibilität von NTH-Empfehlungen in nationalen Ernährungsrichtlinien und Kreislaufwirtschaftsprinzipien (Kapitel 5). Wir wählten fünf Länder aus, die unterschiedliche Regionen und Rahmenbedingungen in Europa repräsentieren (Bulgarien, Malta, die Niederlande, Schweden und die Schweiz). Für jedes dieser Länder untersuchten wir, ob die NTH-Empfehlungen nur mit dem national verfügbaren BNO erfüllt werden können. Wir verwendeten ein Ressourcenallokationsmodell, das BNO verschiedenen Tierproduktionssystemen zuordnet, um verschiedene Ernährungsergebnisse für den Menschen zu maximieren. Die Ernährungsergebnisse wurden so gewählt, dass verschiedene (Gruppen von) Nährstoffen maximiert wurden - d.h. Proteine, Fettsäuren, Mineralien und Vitamine. Anschliessend berechneten wir die Treibhausgasemissionen und die Landnutzung der verschiedenen Optimierungsszenarien. Wir fanden heraus, dass alle Länder erhebliche Mengen an NTH empfehlen, die mit BNO allein nicht erreicht werden können. Die maximal erreichbaren Proteingehalte mit BNO reichten von 15,9 g Protein pro Kopf und Tag (Malta) bis 38,9 g Protein pro Kopf und Tag (Schweiz). Die Zusammensetzung der NTH auf Basis von BNO unterschied sich deutlich von der in den Leitlinien empfohlenen Zusammensetzung der NTH - konnte aber je nach gewähltem Ernährungsschwerpunkt angepasst werden. Zusammenfassend haben wir festgestellt, dass eine Begrenzung der NTH auf BNO zu einem klimafreundlicheren Ernährungssystem mit stärkerem Fokus auf Kreislaufwirtschaft führen würde, wofür eine Überarbeitung und Absenkung der NTH-Empfehlungen erforderlich wäre.

Die EAT-Lancet-Kommission hat kürzlich eine gesunde Referenzdiät herausgegeben, die die planetaren Grenzen respektiert. Wir untersuchten die Vereinbarkeit von NTH in dieser gesunden Referenzdiät mit den Prinzipien der Kreislaufwirtschaft für die Europäischen Union EU-28 (Kapitel 6). Im Vergleich zu den untersuchten nationalen Ernährungsrichtlinien empfehlen die EAT-LancetErnährungsrichtlinien wesentlich geringere Mengen an NTH. Aufgrund dessen konnte die Gesamtmenge an NTH-Protein eingehalten werden, obwohl auch hier die genaue Zusammensetzung nicht eingehalten werden konnte. Insbesondere enthalten die EAT-Lancet-Ernährungsrichtlinien grössere Mengen an Geflügelfleisch als an Rind- und Schweinefleisch, während mit dem verfügbaren BNO hauptsächlich Milch, Rind- und Schweinefleisch hergestellt werden konnten. Wie bei den untersuchten nationalen Ernährungsrichtlinien haben wir festgestellt, dass sowohl die Treibhausgasemissionen als auch der Flächenverbrauch erheblich reduziert werden könnten. Insgesamt müssen die Zusammensetzung der NTH und die zulässige Substituierbarkeit sorgfältig bedacht werden, um eine angepasste gesunde Ernährung aus zirkulären Ernährungssystemen zu erreichen.

In Kapitel 7 wurde der Beitrag der vorgestellten Ergebnisse zu einer differenzierteren Debatte über die Rolle von NTH in nachhaltigeren Ernährungssystemen diskutiert. Unsere Ergebnisse zeigen deutlich, dass Lösungen für nachhaltigere Ernährungssysteme von den Nachhaltigkeitsdimensionen und den einbezogenen spezifischen Indikatoren abhängen. Daher müssen die Ergebnisse für Strategien zu nachhaltigeren Ernährungssystemen im Kontext der gewählten Indikatoren interpretiert und die damit verbundenen Annahmen, Schwerpunkte und Auslassungen kritisch diskutiert werden. Obwohl die Substitution von NTH durch pflanzliche Alternativen viele 
Synergien für die Umwelt und die menschliche Gesundheit aufzeigte, fanden wir auch Zielkonflikte aufgrund von erhöhten sozialen Risiken. Dieses Ergebnis legt nahe, dass eine solche Ernährungsumstellung von geeigneten Massnahmen begleitet werden muss, die diese potenziellen Risiken adressieren. Um Kompatibilitätsprobleme bei der Integration von NTH-Empfehlungen aus Ernährungsrichtlinien mit den Prinzipien der Kreislaufwirtschaft zu umgehen, muss ausserdem die ernährungsphysiologische Funktion von NTH geklärt werden. Schliesslich hat die Diskussion über die Auswirkungen auf die verschiedenen Horizonte, die in dieser Arbeit verwendet wurden, gezeigt, dass der gewählte Horizont die Anforderungen für einen Wandel hin zu nachhaltigeren Ernährungssystemen bestimmt, und damit auch die potenzielle Rolle von NTH in diesem. Zusammenfassend lässt sich sagen, dass die Rolle von NTH in nachhaltigeren Ernährungssystemen vielfältig sein kann - und in hohem Masse von den geforderten Ernährungsbeiträgen sowie von definierten Prioritäten für verschiedene Dimensionen der Nachhaltigkeit abhängt. 


\section{Acknowledgements}

During my time as a PhD student, competent supervisors, motivating work colleagues, and my friends and families accompanied me. Thanks to all of you for making my PhD such an exciting journey that I would not want to miss!

First, I would like to thank the Swiss National Science Foundation SNSF and the Research Institute of Organic Agriculture FiBL in Switzerland for financing this $\mathrm{PhD}$ project.

Special thanks go to my four supervisors: Imke de Boer, Hannah van Zanten, Adrian Muller and Christian Schader. You were all committed to finding a common ground where we can plant our seeds on and see the plants grow and flourish. You also offered me countless opportunities to develop and demonstrate my abilities. Imke, I really appreciate your ability to explain complicated things in an accessible fashion, and to bring in clear lines of argumentation. I always looked forward to my stays in Wageningen, where you immediately made me feel welcome. Hannah, you are inspiringly motivated and dedicated to your work. I greatly appreciate your visions and your ability to see storylines. Thank you for giving me the opportunity to continue doing research in this flourishing area. Adrian, your way of thinking around many edges in an unbiased way fascinates me a lot. Thank you so much for always taking the time to think carefully about ideas and possible solutions with me. Christian, you often spotted weak points quickly, which helped to get issues resolved. Many thanks for leading the project my $\mathrm{PhD}$ was associated to successfully. It was a great pleasure to be supervised by you four!

A big thank you goes to all my co-authors for their important contributions to the work in this thesis, and to the partners in the NRP69-project. Sarah, Birgit, Christine, Carsten, Julia and Jean-Philippe, it was a pleasure to work with you. Thanks to Giulia, Sabine and Angelika for your insights into the human health aspects of food choices. Renée, you were an irreplaceable help in data collection and interpretation. Ben, it is great to exchange on modelling issues with you.

Both in Switzerland and in the Netherlands I was surrounded by great work colleagues. To my FiBL colleagues, thanks for the supporting environment, all the coffee breaks, walks with a strict protocol, boulder sessions, jogging as well as ski tours and so much more. Thanks to Mathias, Bernadette, Simon and Stefan for your support with organisational aspects. Special thanks go to Eva - you were my twin-PhD and there for me in all the good and less good moments, for which I cannot thank you enough. Lina and Anja, thank you for being such engaged and loyal colleagues, and for contributing to our many PhD activities at FiBL. Also big thanks to Rebekka for making an unforgettable summer in Basel possible. I am very thankful to the colleagues at the Animal Production Systems group for providing a great environment during my sporadic visits to Wageningen. Special thanks go to Ollie for your excellent work that builds the basis of so much to come. I enjoyed our (h)Olliedays a lot, and was excited to welcome both you and Eva in Finland last summer. It is an honour to have you two as my paranymphs! Thanks to the colleagues at the Farming Systems Ecology group for welcoming me cordially during these homeoffice times, and special thanks to Wolfram and Felipe, for making our many online meetings a time I am always looking forward to. 
I am truly grateful to my friends for taking me - and my thoughts - to other places whenever needed. Lisa and Marlen, you are always there for me, for which I am incredibly grateful. Lisa, thanks for being by my side for uncountable adventures during the last 15 years. Marlen, it is simply a pleasure to spend time with you and your mind full of sparkling ideas. I am honoured to feature your ideas on my cover. Together with Anna, Adi and Remo, thanks for being a genius foxtrail association. Simone and Sabrina, you are such loyal friends since the year we were born. Sara, I always look forward to our outdoor adventures. Aurich and Ellen, thanks for all the korvapuustit, movie walks, and for always welcoming us back to Bern again. Milena, thanks for the inspiring hiking trips. Thanks to all the colleagues that contributed to our unforgettable seed exhibition in Winterthur. Martina, Flo, Conny, Claudia, Celia, Lisa and Dario, studying with you was a pleasure, thanks for still being great friends.

Special thanks go to my family for always supporting me. I am most grateful to my parents Karin and Urs, for your understanding and motivation for all new projects, and for providing a safe haven I am always welcome to. Mama, thanks for introducing me to the fascinating world of (collecting) mushrooms. Thanks to my sister Gabi, who does not need many words to be described: she is simply the best in the world! To my grandparents Margrit, Walter and Erika, for always being a source of inspiration to me and for accompanying me through all these years. Götti, Jane, Noah, Sean and my dearest Claire, I always long for your visits, thanks for bringing some Mosambik-vibes to our family. Gotta, Markus and Sämi, it is a pleasure to go on skitours, hikes or climbs with you, where I always learn a lot about forests, botany and wildlife. Next to my Swiss family I also received a Finnish family, which I very much appreciate. Helena, Pertti, Elina, Matti, Olavi, Johanna and Jyrki: thanks for welcoming me to your family in the most Moomin-like way. Summerdays at the mökki in your company are a true source of inspiration. Final thanks go to Antti, my husband and favourite partner for all adventures - from exploring mountains with skis or ropes to sharing our homeoffice. Each day I am incredibly grateful for having you by my side. During my PhD time you always motivated and supported me, and most importantly, kept believing in me. 


\section{About the author}

Anita Frehner was born in Heiden, Switzerland, in 1991. In 2013, she obtained her BSc in Economics and General Ecology from the University of Bern. After working at the Bern University of Applied Sciences for a year, she started the Master's programme in Climate Sciences with special qualification in Economics at the University of Bern, from which she graduated in 2016. In her master thesis, Anita focused on the potential of climate service for smallholder farmers in Peru, in collaboration with MeteoSwiss and SENAMHI. Next to this, she worked as a research assistant for the Swiss Federal Institute of Technology Lausanne EPFL in a project that assessed the economic impacts of climate

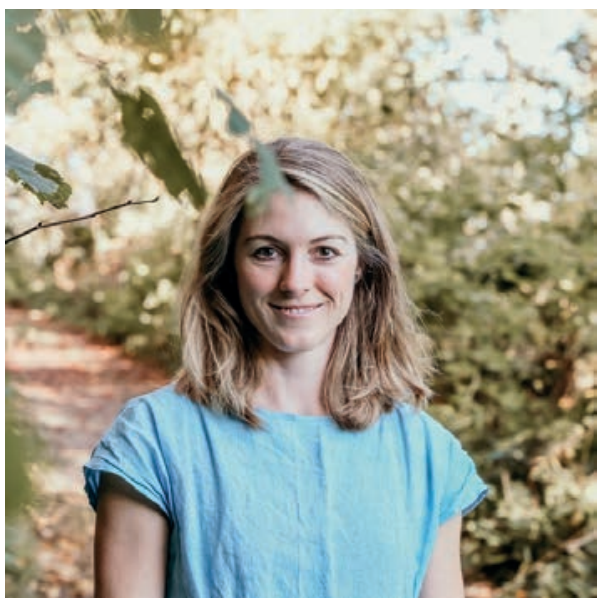
change for Switzerland, where she covered the agricultural sector. Following her interest in research on sustainable practices on the nexus of agriculture, human consumption, and the environment, she started her PhD project in 2017 with the Socioeconomics department at the Research Institute of Organic Agriculture FiBL in Switzerland and with the Animal Production Systems group at Wageningen University and Research in the Netherlands. This $\mathrm{PhD}$ project was associated to the Swiss National Science Foundation project 'NRP69: Trade-offs and synergies of healthy and sustainable nutrition', which aimed at integrating human health and sustainability aspects when analysing diets, and developing recommendations towards better performing dietary patterns and modes of production. Currently, Anita works as a postdoctoral researcher in the Farming Systems Ecology group at Wageningen University and Research in the Netherlands and as a scientific collaborator in the Socioeconomics department at the Research Institute of Organic Agriculture FiBL in Switzerland. 


\section{Publications}

\section{Refereed scientific journals}

Frehner, A., Muller, A., Schader, C., De Boer, I.J.M., Van Zanten, H.H.E., 2020. Methodological choices drive differences in environmentally-friendly dietary solutions. Global Food Security 24, 100333.

Frehner, A., Van Zanten, H.H.E., Schader, C., De Boer, I.J.M., Pestoni, G., Rohrmann, S., Muller, A., 2021. How food choices link sociodemographic and lifestyle factors with sustainability impacts. Journal of Cleaner Production 300, 126896.

Frehner, A., Cardinaals, R.P.M., De Boer, I.J.M., Muller, A., Schader, C., Van Selm, B., Van Hal, O., Pestoni, G., Rohrmann, S., Herrero, M., Van Zanten, H.H.E., 2021. Integrating circularity principles with animal-source food recommendations in national dietary guidelines: Nutritional and environmental consequences. Submitted.

Frehner, A., De Boer, I.J.M., Muller, A., Van Zanten, H.H.E., Schader, C., 2021. Consumer strategies towards a more sustainable food system: Insights from Switzerland. Submitted.

Kopainsky, B., Frehner, A., Muller, A., 2020. Sustainable and healthy diets: Synergies and trade-offs in Switzerland. Systems Research and Behavioral Science 1-20.

Krieger, J. P., Pestoni, G., Frehner, A., Schader, C., Faeh, D., Rohrmann, S., 2020. Combining recent nutritional data with prospective cohorts to quantify the impact of modern dietary patterns on disability-adjusted life years: A feasibility study. Nutrients 12(3), 833.

Van Selm, B., Frehner, A., De Boer, I.J.M., Van Hal, O., Hijbeek, R., Van Ittersum, M.K., Talsma, E.F., Lesschen, J.-P., Hendriks, C.M.J., Herrero, M., Van Zanten, H.H.E., 2021. The compatibility of animal-source food and circularity in healthy European diets. Submitted.

Vöhringer, F., Vielle, M., Thalmann, P., Frehner, A., Knoke, W., Stocker, D., Thurm, B., 2019. Costs and benefits of climate change in Switzerland. Climate Change Economics 10(02), 1950005.

\section{Refereed conference papers}

Frehner, A., Muller, A., Schader, C., De Boer, I.J.M., Van Zanten, H.H.E., 2018. Environmental impact assessment of dietary scenarios: a review on methods. In Proceedings of the 11th International Conference on Life Cycle Assessment of Food, pp. 559-562.

Frehner, A., Van Zanten, H.H.E., Schader, C., de Boer, I.J.M., Muller, A., 2020. Drivers of global warming potential and diet quality of Swiss food consumption. In Proceedings of the 12th International Conference on Life Cycle Assessment of Food, pp. 263-268.

Schader, C., Frehner, A., Muller, A., Nathani, C., Kopainsky, B., Alig, M., Rohrmann, S., Brombach, C., 2020. Trade-offs and synergies between human health and sustainability of Swiss dietary scenarios. In Proceedings of the 12th International Conference on Life Cycle Assessment of Food, pp. 269272. 


\section{Conference posters}

Frehner, A., De Boer, I.J.M., Cardinaals, R.P.M., Muller, A., Schader, C., Van Zanten, H.H.E., 2020. Assessing food-based dietary guidelines from a resource use perspective. Poster at: 12th International Conference on Life Cycle Assessment of Food. https://orgprints.org/id/eprint/39170/

\section{Scientific reports}

Stolze, M., Schader, C., Muller, A., Frehner, A., Kopainsky, B., Nathani, C., Brandes, J., Rohrmann, S., Krieger, J.-P., Pestoni, G., Brombach, C., Flückiger, S., Stucki, M., Frischknecht, R., Alig, M., Hayer, A., 2019. Sustainable and healthy diets: Trade-offs and synergies. Final scientific report. https://orgprints.org/id/eprint/36918/ 


\section{Education certificate}

Completed training and supervision plan ${ }^{1}$

3 ECTS

Basic package

2017

WIAS introduction day

2017

Research Integrity \& Ethics and Animal Science

2017

Disciplinary competences

16.8 ECTS

Writing research proposal

Healthy and sustainable diets

Integrated land use modelling (BOKU) 2018

Economic modelling with the GAMS software (Universität Giessen) 2018

Environmental impact assessment of livestock systems 2019

Summer school: food systems modelling (UFZ Leipzig) 2019

Professional competences

4.1 ECTS

Scientific writing (FiBL) 2019

Writing grant proposals

Reviewing a scientific manuscript 2020

Presentation skills

4 ECTS $^{2}$

Poster presentation, LCA Food, Bangkok, Thailand 2018

Oral presentation, AgroEcoNet, ETH Zürich 2019

Oral presentation, LCA Food, online 2020

Poster presentation, LCA Food, online 2020

Oral presentation, Swiss Public Health Forum, online 2020

\footnotetext{
${ }^{1}$ With the listed activities, the PhD candidate complied with the educational requirements of Wageningen Institute of Animal Sciences (WIAS), a Wageningen University \& Research graduate school. One ECTS equals a study load of 28 hours.

${ }^{2}$ Listed activities provided more ECTS than granted by WIAS regulations.
} 
Supervision BSc thesis (1) and MSc thesis (1)

2018-2020

Supervision internships (2)

2017, 2019

Lecturer, Environmental impacts of our diets

2020, 2021

Lecturer, The role of animals in circular food systems

2020, 2021

Course assistance, Introduction to GAMS

Lecturer, Arguing for sustainable food systems

Course assistance, Quantitative analysis of land use systems

2021 


\section{Colophon}

The research described in this thesis was financially supported by the Swiss National Science Foundation SNSF (project 4069-166765) and the Research Institute of Organic Agriculture FiBL in Switzerland.

Cover design by Marlen Kühn

Printed by ProefschriftMaken 

